\section{Pacific Northwest} National Laboratory

Operated by Battelle for the

U.S. Department of Energy

\title{
Contaminants of Potential Concern in the 300-FF-5 Operable Unit: Expanded Annual Groundwater Report for Fiscal Year 2004
}

R. E. Peterson, Editor

$\begin{array}{lll}\text { E. J. Freeman } & \text { C. J. Murray } & \text { P. D. Thorne } \\ \text { M. J. Truex } & \text { V. R. Vermeul } & \text { M. D. Williams } \\ \text { S. B. Yabusaki } & \text { J. M. Zachara } & \text { J. L. Lindberg } \\ \text { J. P. McDonald } & & \end{array}$

March 2005

Prepared for the U.S. Department of Energy under Contract DE-AC05-76RL01830 


\title{
DISCLAIMER
}

This report was prepared as an account of work sponsored by an agency of the United States Government. Reference herein to any specific commercial product, process, or service by trade name, trademark, manufacturer, or otherwise does not necessarily constitute or imply its endorsement, recommendation, or favoring by the United States Government or any agency thereof, or Battelle Memorial Institute.

\author{
PACIFIC NORTHWEST NATIONAL LABORATORY \\ operated by \\ BATTELLE \\ for the \\ UNITED STATES DEPARTMENT OF ENERGY \\ under Contract DE-AC05-76RL01830
}

\begin{abstract}
Printed in the United States of America
Available to DOE and DOE contractors from the

Office of Scientific and Technical Information, P.O. Box 62, Oak Ridge, TN 37831;

prices available from (615) 576-8401.

Available to the public from the National Technical Information Service,

U.S. Department of Commerce, 5285 Port Royal Rd., Springfield, VA 22161
\end{abstract}

This document was printed on recycled paper. 


\section{Contaminants of Potential Concern in the 300-FF-5 Operable Unit: Expanded Annual Groundwater Report for Fiscal Year 2004}

R. E. Peterson, Editor

$\begin{array}{lll}\text { E. J. Freeman } & \text { C. J. Murray } & \text { P. D. Thorne } \\ \text { M. J. Truex } & \text { V. R. Vermeul } & \text { M. D. Williams } \\ \text { S. B. Yabusaki } & \text { J. M. Zachara } & \text { J. L. Lindberg } \\ \text { J. P. McDonald } & & \end{array}$

March 2005

Prepared for

the U.S. Department of Energy

under Contract DE-AC05-76RL01830

Pacific Northwest National Laboratory

Richland, Washington 99352 


\section{Summary}

The Groundwater Performance Assessment Project, managed by the U.S. Department of Energy's Pacific Northwest National Laboratory (PNNL), provides site-specific and site-wide assessments of groundwater conditions for the Hanford Site. The project is responsible for groundwater monitoring, data evaluation and interpretation, and modeling. Staff at PNNL prepared this report to give decision makers additional information on the characteristics of groundwater in the 300-FF-5 Operable Unit, located in the southeast corner of the Hanford Site. The operable unit includes groundwater beneath the 300 Area, and beneath two outlying sub-regions: the 618-11 burial ground and the 316-4 cribs/618-10 burial ground.

In 1996, a record of decision (ROD) stipulated interim remedial action for groundwater affected by releases from 300 Area sources, as follows: (a) continued monitoring of groundwater that is contaminated above health-based levels to ensure that concentrations continue to decrease, and (b) institutional controls to ensure that groundwater use is restricted to prevent unacceptable exposure to groundwater contamination. In 2000, the groundwater beneath the two outlying sub-regions was added to the operable unit. In 2001, the first 5-year review of the ROD found that the interim remedy and remedial action objectives were still appropriate, although the review called for additional characterization activities. This report provides a comprehensive description for many of the results from those activities.

This report satisfies an operations and maintenance plan requirement to provide an expanded annual report on groundwater conditions for fiscal year 2004. Routine annual reporting disseminates information that characterizes current conditions and trends and provides a basis for changes to the monitoring tasks, as appropriate. This supplemental report supplies additional details and serves additional purposes, which include support for the second 5-year review of the ROD and a Phase III Feasibility Study. The report contains several major subsections: (a) current characteristics of contaminants in groundwater, (b) conceptual models for uranium in the 300 Area and tritium in the 618-11 subregion, and (c) progress during the period of interim remedial action.

Current Characteristics. The maximum concentration values for each contaminant of concern (COC) or potential concern (COPC) for the period 1992 through 2004 are used to show where standards are still exceeded and to illustrate trends. Uranium in the 300 Area, and tritium in the 618-11 sub-region, remain at concentrations well above the drinking water standard. Other COPC reveal recent concentrations near or below the standards. For many waste constituents, concentrations remain generally constant or are decreasing. A primary contributor to concentration changes since 1992 appears to be plume migration under natural groundwater flow conditions.

For uranium in the 300 Area, plume maps for June and December of 2002, 2003, and 2004 were used to provide estimates for various plume parameters. Where groundwater exceeds the $30-\mu \mathrm{g} / \mathrm{L}$ drinking water standard, parameters varied as follows: (a) area of plume equals 0.36 to 0.46 square kilometers; (b) volume of contaminated groundwater equals $\sim 294,000$ to $\sim 377,000$ cubic meters; and (c) mass of dissolved uranium equals $\sim 18$ to $\sim 30$ kilograms. Uncertainty in the accuracy of 
these estimates is caused by the need to make certain assumptions, such as the thickness of the contaminated layer. However, for the period evaluated, the trends in parameters suggest a relatively constant level of contamination, but with some variability.

At the 618-11 sub-region, monitoring results since 1999 show decreasing tritium concentrations at wells closest to the source and variable concentrations at wells along the downgradient migration pathway. This plume has not reached the Energy Northwest water supply wells, nor the Columbia River. At the 316-4/618-10 sub-region, COPC are currently at levels below the drinking water standards, except for very recent samples from two wells near the 316-4 cribs excavation site that show concentrations near the 30-ug/L standard for uranium.

A revised strategy for categorizing waste constituents in groundwater as a COC or COPC, along with the implications for remedial actions and regulatory decisions, is proposed. As a result, the lists developed during the remedial investigation have been shortened, primarily because of improving conditions and lack of evidence suggesting unacceptable risk.

Conceptual Site Model for 300 Area Uranium. The 300 Area uranium plume can be characterized as persistent, i.e., the area and concentrations have remained similar to early 1990 conditions. There has been variability in spatial and temporal distribution patterns, primarily as a consequence of (a) cessation of liquid waste disposal to the ground, (b) large-scale source excavation activities, (c) unusually high and prolonged water table conditions during 1996 and 1997, and (d) seasonality because of river-stage fluctuations. During the most recent years, the plume appears to be relatively stable, with evidence showing gradual downgradient migration to the Columbia River. The highest concentrations observed currently are along the shoreline, and probably reflect the last significant input from beneath former major waste sites, such as the 316-5 process trenches. Uranium is lost from the plume via discharge to the river and groundwater withdrawal at a water supply well. Some amount of re-supply to the plume is believed to occur as a consequence of long-term release of uranium that has been sequestered on vadose zone and aquifer solids.

The mobility of uranium and controls on dissolved concentrations are influenced by the geochemistry of the original waste effluent, the receiving sediment, and pore fluids, all of which vary in the 300 Area environment. The compositional and spatial variability leads to complexity in computer models designed for predicting plume behavior. The heterogeneity in conditions also drives the need for more field data on the locations, inventory, and geochemical characteristics of uranium in potential source zones.

Conceptual Site Model for 618-11 Tritium. The tritium plume associated with the 618-11 sub-region has apparently been created by episodic release of tritium gas from irradiated materials in the burial ground. The gas interacts with moisture in the vadose zone and eventually impacts groundwater. The high concentrations in groundwater observed near the burial ground during 1999 to 2000 have declined in recent years, and the plume shows evidence of slow, downgradient migration. The plume has not reached Energy Northwest water supply wells nor the Columbia River. In the absence of tritium re-supply to the plume, concentrations will decrease because of transport processes (i.e., dispersion) and radioactive decay. The amount of tritium in the environment will decrease because of radioactive decay alone. Initial results from a computer simulation of plume behavior, under one of several scenarios being modeled, suggest that concentrations in the plume will be below the drinking water standard in several tens of years, assuming no further re-supply to the plume. 
Progress During Interim Remedial Action. Interim remedial action for the operable unit includes continued monitoring of groundwater that is contaminated above health-based levels and institutional controls to ensure that groundwater use is restricted. During the past 5 years, monitoring activities have been expanded to serve two purposes: (1) to verify that concentrations for contaminants of concern continue to decrease, as anticipated from the results of the remedial investigation, and (2) to evaluate the interim remedy in light of more recent U.S. Environmental Protection Agency (EPA) guidance for including monitored natural attenuation (MNA) as a remedy ${ }^{1}$. Groundwater monitoring has included use of nearly all available monitoring wells and newly installed aquifer sampling tubes at the shoreline. Additional efforts have been conducted by other Hanford Site programs to monitor conditions along the 300 Area shoreline. An analysis of contaminant concentration trends during the past 12 years has revealed information that helps to limit the lists of COC or COPC, and to provide information to make estimates for how long it will take for contaminants to attenuate to acceptable levels under natural conditions. Results of all these activities are summarized in a format that is intended to support the second 5-year review of the ROD.

Because natural features and processes play a role in reducing the level of contamination in groundwater to some degree, an analysis of current EPA guidance for including MNA as a contributing or sole remedial action alternative is presented. EPA's three-tiered approach for evaluating the suitability of MNA as a remedy is discussed using site-specific information for the 300-FF-5 Operable Unit, along with a discussion of key considerations for determining whether MNA is appropriate at this site. The analysis suggests that the interim remedy currently selected in 1996 is consistent with subsequent EPA guidance (1999), and that 300-FF-5 characteristics are favorable for including MNA as a remedial action alternative.

\footnotetext{
${ }^{1}$ In 1996 when the ROD was prepared, monitored natural attenuation had not yet been formally defined by EPA as a remedial action alternative. The definition was subsequently included in guidance published in 1999.
} 


\section{Acronyms}

$\begin{array}{ll}\text { CERCLA } & \text { Comprehensive Environmental Response, Compensation, and Liability Act } \\ \text { COC } & \text { contaminant of concern } \\ \text { COPC } & \text { contaminant of potential concern } \\ \text { DaVE } & \text { data viewer and evaluator (database) } \\ \text { DOE } & \text { U.S. Department of Energy } \\ \text { Ecology } & \text { Washington State Department of Ecology } \\ \text { EPA } & \text { U.S. Environmental Protection Agency } \\ \text { FY } & \text { fiscal year } \\ \text { MNA } & \text { monitored natural attenuation } \\ \text { NRC } & \text { U.S. Nuclear Regulatory Commission } \\ \text { RCRA } & \text { Resource Conservation and Recovery Act } \\ \text { ROD } & \text { record of decision } \\ \text { SCM } & \text { surface complexation model }\end{array}$




\section{Acknowledgments}

The authors wish to thank Pacific Northwest National Laboratory staff who provided invaluable assistance in the important areas of data processing and model data input preparation and final report preparation. This report includes contributions from numerous individuals who have provided early drafts of text that will ultimately appear in separate published reports. In many instances, their drafts have been edited and/or summarized by the editor for this report, R. E. Peterson, who takes full responsibility for the accuracy of such changes. The extensive data summaries were completed by J. T. Rieger, and the uranium plume maps and plume parameter estimates were prepared by C. A. Newbill. Final editorial polish and document production were done by L. F. Morasch, K. R. Neiderhiser, and L. M. Andor, who provided exceptional help under a very tight schedule. 


\section{Contents}

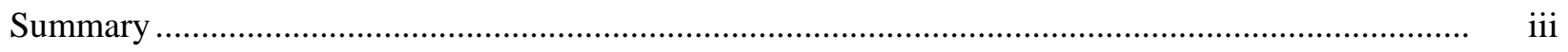

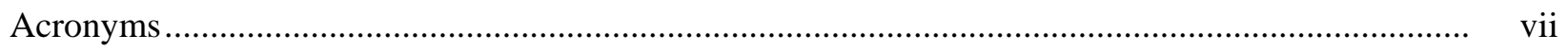

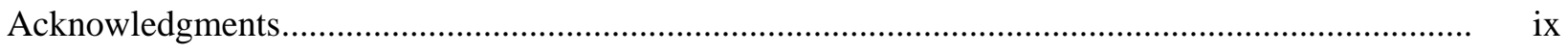

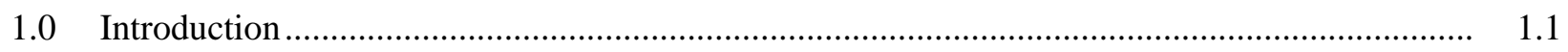

1.1 300-FF-5 Record of Decision and First 5-Year Review............................................... 1.2

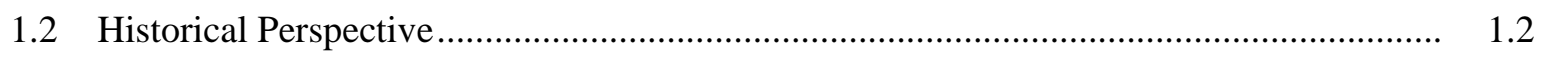

1.3 Organization of the Expanded Report for Fiscal Year 2004 ......................................... 1.4

2.0 Current Characteristics ...................................................................................... 2.1

2.1300 Area Contaminants of Concern or Potential Concern ............................................ 2.1

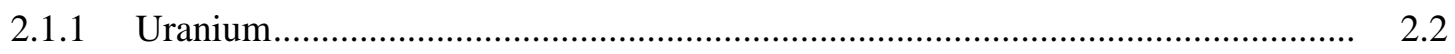

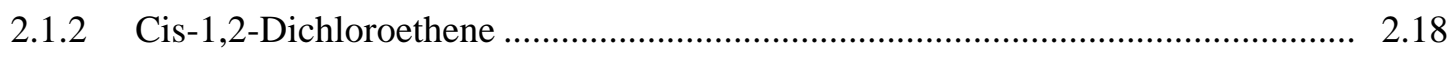

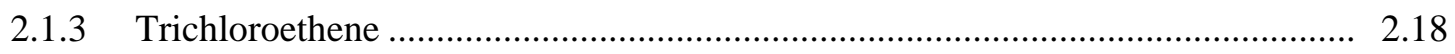

2.1.4 Other Constituents of Potential Concern.......................................................... 2.19

2.2 618-11 Burial Ground Contaminants of Concern or Potential Concern ........................... 2.34

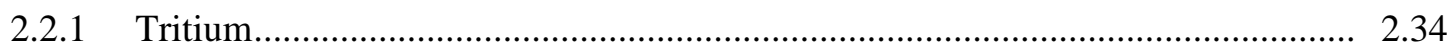

2.2.2 Other Contaminants of Potential Concern..................................................... 2.34

2.3 316-4 Cribs/618-10 Burial Ground Contaminants of Potential Concern .......................... 2.41

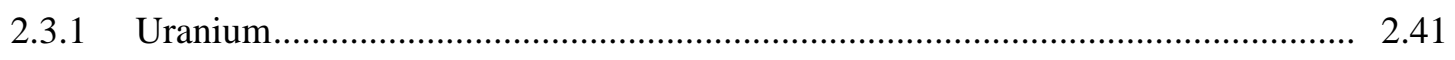

2.3.2 Tributyl Phosphate ............................................................................. 2.41

2.3.3 Other Contaminants of Potential Concern....................................................... 2.42

2.4 Proposed Updates to Lists for Contaminants of Concern or Potential Concern................ 2.47

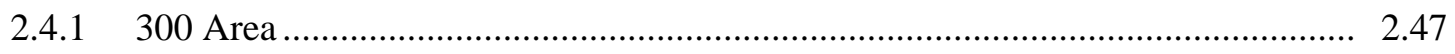

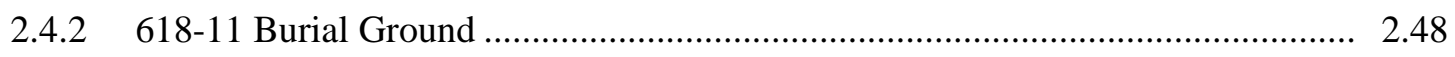

2.4.3 316-4 Cribs and 618-10 Burial Ground...................................................... 2.50

3.0 Conceptual Model for Uranium at the 300 Area ................................................................ 3.1

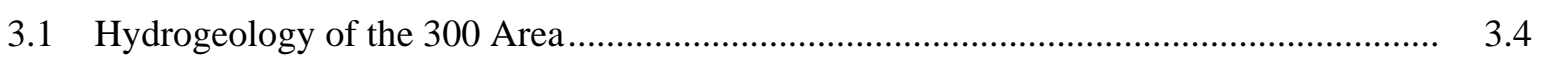

3.1.1 Hydrogeologic Framework for the 300 Area ................................................... 3.4

3.1.2 Groundwater Flow Conditions ....................................................................... 3.6

3.2 Groundwater Flow and Transport Modeling ............................................................ 3.10

3.2.1 Hydraulic Parameter Estimation Model ......................................................... 3.11

3.2.2 Prediction Flow Model .................................................................................. 3.13

3.2.3 Uranium Transport Model ......................................................................... 3.14 
3.3 Trends in Plume Parameters ................................................................................... 3.15

3.3.1 Geostatistical Methods to Characterize Plumes ............................................... 3.15

3.3.2 Representative Concentrations Over Time for the Uranium Plume..................... 3.16

3.3.3 Principal Assumptions for the Geostatistical Analysis....................................... 3.20

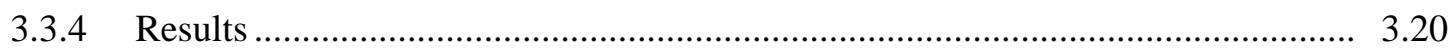

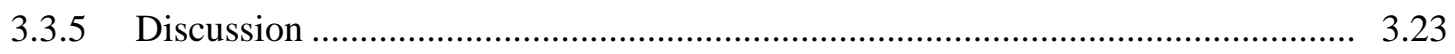

3.4 Geochemistry and Mobility of Uranium in 300 Area ................................................. 3.25

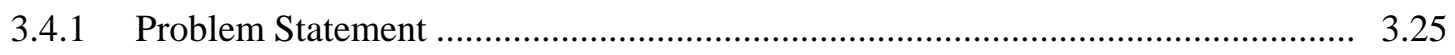

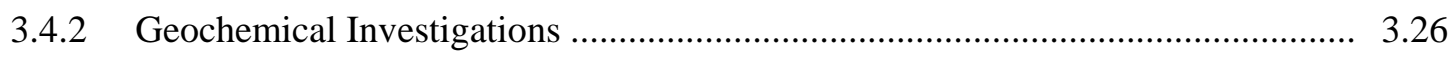

3.4.3 Preliminary Geochemical Conceptual Model ................................................ 3.26

3.4.4 Implications of Research Results to the 300 Area Uranium Plume ..................... 3.28

3.5 Key Aspects of Conceptual Model for 300 Area Uranium Plume ................................... 3.29

4.0 Conceptual Model for Tritium at 618-11 Burial Ground ....................................................... 4.1

4.1 Hydrogeologic Conceptual Model ............................................................................. 4.1

4.1.1 Sedimentary Geologic Units ..................................................................... 4.1

4.1.2 Hydraulic Properties of Hydrogeologic Units.................................................. 4.5

4.2 618-11 Tritium Plume Conceptual Model................................................................... 4.7

4.3 618-11 Tritium Plume Trends ................................................................................ 4.10

4.4 Groundwater Flow and Transport Modeling ........................................................... 4.14

5.0 Progress During Interim Remedial Action................................................................... 5.1

5.1 Operable Unit Monitoring and Characterization .......................................................... 5.1

5.2 Interim Remedial Action Objectives ...................................................................... 5.2

5.2.1 Interim Action Requirements ....................................................................... 5.3

5.2.2 Interim Action: 300 Area ..................................................................... 5.3

5.2.3 Interim Action: 618-11 Burial Ground.......................................................... 5.5

5.2.4 Interim Action: 316-4 Cribs/618-10 Burial Ground......................................... 5.6

5.3 EPA Guidelines for Monitored Natural Attenuation as a Remedy ................................. 5.7

5.3.1 Key Principals for Selection of Remedial Measures ......................................... 5.8

5.3.2 Approach for Evaluating Monitored Natural Attenuation as a Remedy .............. 5.9

5.3.3 Key Considerations as to Appropriateness of MNA at a Particular Site.............. 5.11

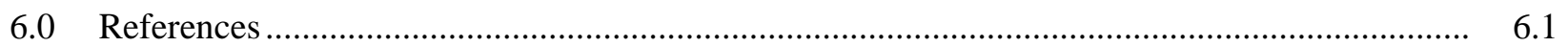

Appendix A - Trend Charts of Uranium Concentrations at 300 Area Wells

Appendix B - Tri-Party Agreement Milestone M-016-83, March 11, 2005

Appendix C - Evolution of Regulatory Framework for 300-FF-5 Operable Unit 


\section{Figures}

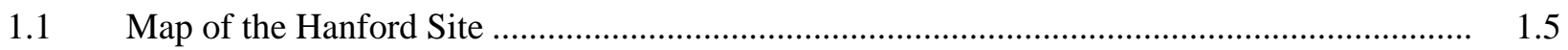

1.2 Locations of Monitoring Wells, Aquifer Tubes, Riverbank Springs, and Facilities at the 300 Area.

1.3 Locations of Monitoring Wells Near the 618-11 Burial Ground and 316-4 Cribs/ 618-10 Burial Ground Sub-Regions

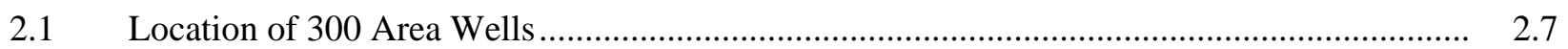

2.2300 Area Uranium Plume, August/September 2001 Conditions .......................................... 2.8

$2.3 \quad 300$ Area Uranium Plume, June 2002 Conditions................................................................ 2.9

2.4300 Area Uranium Plume, December 2002 Conditions....................................................... 2.10

2.5300 Area Uranium Plume, June 2003 Conditions.............................................................. 2.11

2.6300 Area Uranium Plume, December 2003 Conditions....................................................... 2.12

2.7300 Area Uranium Plume, June 2004 Conditions................................................................ 2.13

2.8300 Area Uranium, December 2004 ............................................................................. 2.14

2.9 Uranium Concentrations at Aquifer Tubes Along 300 Area Shoreline ................................. 2.15

2.10 Tritium Plume Downgradient of 618-11 Burial Ground, 2004 Conditions ............................. 2.35

2.11 Tritium Concentrations at Wells Near the 618-11 Burial Ground ......................................... 2.36

2.12 Uranium Concentrations at Wells Near the 316-4 Cribs Excavation Site .............................. 2.44

3.1 Diagram Illustrating Key Components of Conceptual Model for Uranium............................ 3.3

3.2 Index Map to Cross Sections of the 300 Area .................................................................... 3.5

3.3 West-to-East Cross Section Along Flow Path from 300 Area Process Trenches

3.4 North-to-South Cross Section Along Shoreline Wells ...................................................... 3.6

3.5 West-to-East Cross Section Through Central Portion of 300 Area ....................................... 3.6

$3.6 \quad 300$ Area Water-Table Elevation, March 1992 .................................................................. 3.7

$3.7 \quad 300$ Area Water-Table Elevation, May 1992 _.................................................................. 3.8

$3.8 \quad 300$ Area Water-Table Elevation, June 1992 _.................................................................. 3.8

3.9300 Area Water-Table Elevation, September 1992 ............................................................ 3.9

3.10300 Area Water Table Elevation, December 1992 .............................................................. 3.9

3.11 Wells Used in 1993 Animated Contour Maps ....................................................................... 3.11

Plan View of STOMP Model Grid ................................................................................ 3.12

3.13 Maps Showing Locations for Representative Concentration Values and Data

Classes for 300 Area Uranium Plume............................................................................... 3.18

3.14 Box Plots for Uranium Concentration Data for Each Time Period ...................................... 3.19 
3.15 Box Plots Showing Average Water-Level Elevation

3.16 Median Concentration of Uranium in the 300 Area Plume for the Four Time Periods Simulated

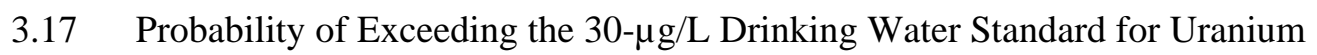
for the Four Periods Studied.

3.18 Box Plots Showing the Simulated Total Mass for the 300 Area Uranium Plume, Assuming a Thickness of 3 Meters

3.19 Box Plots of the Simulated Area Above the Drinking Water Standard for Each Time Period

3.20 Box Plots of the Simulated Length of the Columbia River Shoreline Above the Drinking Water Standards for Each Time Period

4.1 Location of Boreholes Used to Define the Hydrogeologic Conceptual Model

4.2 West-to-East Cross Section through the Model Showing Hydrogeologic Units ..

4.3 North-to-South Cross Section through the Model Showing Hydrogeologic Units 4.5

4.4 Hanford Formation Saturated Thickness Based on the 2001 Water Table. 4.6

4.5 Tritium Plume Downgradient of 618-11 Burial Ground, 2001 Conditions

4.6 Initial Condition Tritium Plume Implemented for the 618-11 Model Compared to Hydrogeologic Layering.

4.7 Tritium Plume Downgradient of 618-11 Burial Ground, 2004 Conditions 4.12

4.8 Tritium Concentration Trend Plot for Monitoring Well 699-13-3A....................................... 4.13

4.9 Tritium Concentration Trend Plot for Monitoring Well 699-13-1E ....................................... 4.13

4.10 Tritium Concentration Trend Plot for Monitoring Well 699-13-0A........................................ 4.14

4.11 618-11 Burial Ground Model Grid ..................................................................................... 4.15

4.12 Comparison of Simulated and Observed Tritium Concentration at Well 699-13-3A.............. 4.16

4.13 Comparison of Simulated and Observed Tritium Concentration at Well 699-13-0A............... 4.16

4.14 Maximum Predicted Concentration throughout the 618-11 Tritium Plume ............................ 4.17

4.15 Predicted Tritium Concentration at the Columbia River Boundary ........................................ 4.18

4.16 Predicted Tritium Concentration at the Energy Northwest Water Supply Wells .................... 4.18

4.17 Tritium Plume at the 618-11 Burial Ground, Initial Conditions in 2001.............................. 4.19

4.18 Tritium Plume at the 618-11 Burial Ground in 2007 .......................................................... 4.19

4.19 Tritium Plume at the 618-11 Burial Ground in 2017 ......................................................... 4.20

4.20 Tritium Plume at the 618-11 Burial Ground in 2027, 3 Years after the Maximum Tritium Concentration Falls Below the Drinking Water Standard 


\section{Tables}

2.1 Maximum Uranium Concentrations at 300 Area Wells...................................................... 2.5

2.2 Area, Volume, and Mass Estimates for the 300 Area Uranium Plume................................... 2.16

2.3 Summary of Uranium Plume Parameters........................................................................... 2.18

2.4 Maximum Cis-1,2-Dichloroethene Concentrations at 300 Area Wells ................................. 2.20

2.5 Maximum Total 1,2-Dichloroethene Concentrations at 300 Area Wells................................ 2.22

2.6 Maximum Trichloroethene Concentrations at 300 Area Wells .............................................. 2.24

2.7 Maximum Tetrachloroethene Concentrations at 300 Area Wells ......................................... 2.26

2.8 Maximum Strontium-90 Concentrations at 300 Area Wells................................................. 2.28

2.9 Maximum Tritium Concentrations at 300 Area Wells...................................................... 2.30

2.10 Maximum Nitrate Concentrations at 300 Area Wells....................................................... 2.32

2.11 Maximum Concentrations for Contaminants of Potential Concern at 618-11

Sub-Region Wells: Group A ..................................................................................... 2.37

2.12 Maximum Concentrations for Contaminants of Potential Concern at 618-11

Sub-Region Wells: Group B .................................................................................. 2.39

2.13 Maximum Concentrations for Contaminants of Potential Concern at 316-4/618-10

Sub-Region Wells: Group A ....................................................................................... 2.43

2.14 Maximum Observed Concentrations for Contaminants of Potential Concern at 316-4/618-10 Sub-Region Wells: Group B ....................................................................... 2.45

2.15 Maximum Concentrations for Site-Wide Plume Constituents at 316-4/618-10

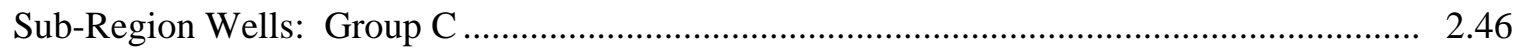

3.1 Representative Uranium Concentrations at Each 300 Area Well for Two-Year

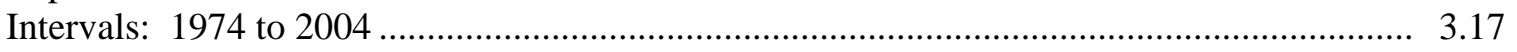

3.2 Mass, Area, and Length of Impacted Shoreline for 300 Area Uranium Plume ...................... 3.25

3.3 Statistical Summary of Representative Uranium Concentrations for Each Time Period.......... 3.25

$4.1 \quad$ Tritium Measured at Discrete Depths in Borehole C3254 ................................................... 4.10 


\subsection{Introduction}

The 300-FF-5 Operable Unit, located in the southeast portion of the Hanford Site, includes groundwater affected by contaminants released from waste sites in three geographic sub-regions of the operable unit: the 300 Area, 618-11 burial ground, and 316-4 cribs/618-10 burial ground (Figure 1.1). A map showing facilities and monitoring well locations for the 300 Area is shown in Figure 1.2, and maps for the outlying sub-regions are shown in Figure 1.3. Groundwater quality in these sub-regions has been affected by releases from sources within the geographic boundaries of the operable unit (i.e., waste sites in the overlying 300-FF-1 and 300-FF-2 Operable Units), and also by contaminants that migrate into the operable unit from sources in the 200 East Area (200-PO-1 Operable Unit) and from sources to the southwest of the 300 Area (1100-EM-1 Operable Unit).

This report satisfies requirements in the Operations and Maintenance Plan for the 300-FF-5 Operable Unit (DOE 2002b) regarding annual reporting on groundwater conditions. The report also satisfies a requirement in Tri-Party Agreement Milestone M-016-83 (Appendix B) to provide detailed descriptions of updated conceptual models for the 300 Area uranium plume and 618-11 burial ground uranium plume.

The purpose for annual reporting is to provide information that can be used for:

1. Describing current conditions.

2. Establishing a basis for changes to the monitoring tasks.

3. Supporting the 5-year reviews of the interim remedial actions.

Each year, information relating to items (1) and (2) above is presented in the annual report of the Groundwater Performance Assessment Project (e.g., Hartman et al. 2005). Section 2.12 of that report covers the 300-FF-5 Operable Unit (Lindberg and Peterson 2005). With regard to item (3), the next 5 -year review of the record of decision (ROD) for the operable unit is being conducted during calendar year 2005, with release of the findings by April 2006. Because of the impending 5-year review, the operations and maintenance plan (DOE 2002b) requires that an expanded annual report be prepared for fiscal year (FY) 2004. The expanded report is to provide increased details regarding interpretation of monitoring results; an assessment of natural attenuation processes, and any other new information pertaining to groundwater conditions that is not includes in the regular annual report.

The following sections present data from long-term groundwater monitoring activities conducted under the Comprehensive Environmental Response, Compensation, and Liability Act (CERCLA) and Resource Conservation and Recovery Act (RCRA) programs. Interpretations of these data with respect to the objectives identified in the ROD (EPA 1996) and the operation and maintenance plan (DOE 2002b) are described. A summary of the results and status of recent laboratory and modeling investigations are also presented, along with updated descriptions for conceptual site models for uranium at the 300 Area and tritium at the 618-11 sub-region. These conceptual site models, along with descriptions of concentration trends for contaminants of potential concern, are intended to provide a technical basis for determining appropriate future actions within the operable unit. 


\section{$1.1 \quad 300-F F-5$ Record of Decision and First 5-Year Review}

The initial ROD for the 300-FF-5 Operable Unit stipulates interim remedial action that imposes restrictions on the use of 300 Area groundwater until such time as health-based criteria are met for uranium, trichloroethene, and cis-1,2-dichloroethene, which are the 300 Area contaminants of concern (COC) as defined by the ROD (EPA 1996, pg. ii). (Note: This ROD is the first Hanford Site ROD for a groundwater operable unit along the river corridor that does not invoke active measures for restoring water quality. ${ }^{1}$ ) As stated in the ROD, the selected interim remedy is:

- "Continued monitoring of groundwater that is contaminated above health-based levels to ensure that concentrations continue to decrease.

- Institutional controls to ensure that groundwater use is restricted to prevent unacceptable exposures to groundwater contamination."

The ROD describes specific remedial action objectives that involve (a) protecting human and ecological receptors from exposure to contaminants in groundwater, and (b) minimizing future impacts to groundwater resources from waste sites. Protection of the Columbia River from contaminants carried by groundwater is an objective, with the criteria for protection as listed in the Washington State Surface Water Quality Standards (WAC 173-201A).

In 2000, the ROD was modified by an explanation of significant difference (EPA 2000) that expanded the geographic scope for the operable unit to include two outlying sub-regions to the northwest of the 300 Area. The interim remedial action and remedial action objectives remained the same as stated in the initial ROD, with the exception of addressing several different contaminants of potential concern (COPC) in the new sub-regions. The northernmost of the two sub-regions contains the 618-11 burial ground, where the COPC is tritium. The second outlying sub-region contains the 316-4 cribs and 618-10 burial ground, where the COPC are uranium and tributyl phosphate. ${ }^{2}$

The first 5-year review of the ROD (EPA 2001) found that the selected interim remedy and remedial action objectives were still appropriate. However, additional actions were specified that called for more characterization of the contamination problems, to include (a) expanded monitoring at the river shoreline, and (b) an assessment of the effectiveness of natural attenuation as a remedy. The expanded characterization and assessment work is described in a revised operations and maintenance plan, which was published in 2002 (DOE 2002b), along with an updated sampling and analysis plan (DOE 2002a).

\subsection{Historical Perspective}

Facilities in the 300 Area of the Hanford Site were primarily involved with fabrication of nuclear fuel for plutonium production, which included some research and development activities, during the period

\footnotetext{
${ }^{1}$ RODs for interim remedial action are in place for the 100-NR-2, 100-KR-4, and 100-HR-3 Operable Units that specify pump-and-treat systems for specific contaminants of concern. RODs have not yet been prepared for other contaminants of potential concern in those operable units, nor have RODs been prepared for the 100-BC-5, 100-FR-3, and 200-PO-1 Operable Units, as of March 2005.

${ }^{2}$ Because a remedial investigation and baseline risk assessment of groundwater associated with these outlying subregions has not been conducted, no contaminants of concern have yet been formally identified. Therefore, waste constituents being monitored will be referred to in this report as contaminants of potential concern.
} 
spanning the startup of Hanford reactors in 1944 through the late 1980s (Young and Fruchter 1991). The range of activities produced a wide variety of waste streams that contained chemical and radiological constituents (Gerber 1992; Deford et al. 1994). Since the early 1990s, extensive remediation of liquid waste disposal sites and solid waste burial grounds has taken place. As of March 2004, most liquid waste disposal sites, which are located in the north half of the 300 Area, have been excavated, backfilled, and the ground surface restored. Some unknown amount of contamination remains in the vadose zone beneath the lower extent of the excavation activities. Additional contamination may also remain beneath buildings and facilities in the southern portion of the 300 Area, where decontamination and decommissioning activities have not yet taken place.

Groundwater beneath the 300 Area and the two outlying geographic sub-regions (618-11 burial ground and 316-4 cribs/618-10 burial ground) contain contaminants from past-practices disposal activities at concentrations that exceed the U.S. Environmental Protection Agency (EPA) standards for drinking water supplies. The CERCLA ROD for interim action associated with groundwater (EPA 1996; EPA 2000) involves institutional controls on the use of groundwater and continued monitoring to establish trends in the level of contamination. (Note: The 300-FF-5 Operable Unit, a groundwater operable unit, includes the water and solids that constitute the aquifer.)

Uranium is the most prominent waste constituent remaining in the environment, and it has persisted in waste sites and groundwater during the years following the shutdown of most fuel fabrication activities and cessation of liquid effluent disposal to the ground. Uranium in soluble form is of concern for chemical toxicity, as well as for radiological exposure, although the concentrations in groundwater for chemical toxicity are lower than those associated with exceeding radiological dose standards. Specific criteria on the toxicity to freshwater aquatic organisms are not currently established, so by default, the criteria for the protection of aquatic organisms are the same as those applied for protection of human health. The EPA's maximum contaminant level in groundwater for drinking water supplies is currently $30 \mu \mathrm{g} / \mathrm{L}$ uranium, measured as total uranium in the water sample. During the remedial investigation in the early 1990s and the development of the initial ROD, the proposed standard for uranium was $20 \mu \mathrm{g} / \mathrm{L}$.

Additional waste constituents present in groundwater beneath the 300 Area include volatile organic compounds, which resulted from disposal of liquid waste generated in the 300 Area facilities. These constituents include cis-1,2-dichloroethene, trichloroethene, and tetrachloroethene. Also, tritium, nitrate, technetium-99, and trichloroethene migrate into the 300-FF-5 Operable Unit from source areas to the northwest and southwest. At the outlying 618-11 waste site, a tritium plume, whose source is assumed to be releases from irradiated materials in the burial ground, is being monitored. At the 316-4/618-10 waste sites, uranium and tributyl phosphate are being monitored. Those two constituents were discharged to the cribs during the very early operations at 300 Area facilities, and residual amounts remain in the vadose zone, as revealed by recent excavation of the 316-4 cribs. No releases from the 618-10 burial ground are known to have impacted groundwater.

During the period of interim remedial action, monitoring and characterization of the various contaminant plumes continues, with one objective being to show how the level of contamination changes with time. The remedial investigation (DOE 1995) found evidence to suggest that levels for uranium would decrease with time (i.e., the plume would attenuate) because of natural processes, such as dispersion. A prediction was offered that concentrations of uranium in groundwater would decrease to the proposed drinking water standard or lower in 3 to 10 years from 1993. This led to anticipating that natural processes would have a role in future decisions regarding remedial action alternatives for groundwater. 
The phrase "natural attenuation processes" is defined in EPA's guidance for including natural processes when considering remedial action alternatives (EPA 1999, pg. 3). The guidance includes the following statements describing natural attenuation processes as:

“...a variety of physical, chemical, or biological processes that, under favorable conditions, act without human intervention to reduce the mass, toxicity, mobility, volume, or concentration of contaminants in soil or groundwater. These in situ processes include biodegradation; dispersion; dilution; sorption; volatilization; radioactive decay; and chemical or biological stabilization, transformation, or destruction of contaminants.”

The expanded groundwater report for fiscal year 2004 considers monitoring results for the period 1992 to 2004. During this period of time, actions that may have impacted the 300-FF-5 Operable Unit included the expedited response action that occurred during the early 1990s, cessation of liquid discharges to the remaining land disposal facilities with the startup of the Treated Effluent Disposal Facility in 1994, extensive source remedial actions involving large-scale excavations of liquid waste disposal sites and solid waste burial grounds during the mid-1990s, and unusually high and prolonged water-table elevations during 1996 and 1997.

\subsection{Organization of the Expanded Report for Fiscal Year 2004}

This expanded report starts with a detailed summary of current conditions and trends for contamination indictors at each of the three geographic sub-regions within the 300-FF-5 Operable Unit (Chapter 2). Tables are included that show maximum concentrations by calendar year for the period 1992 to 2004. Plume maps and trends charts are used where necessary to illustrate key phenomena. Uranium concentration trend charts for each 300 Area well that monitors the uppermost hydrologic unit are included with this report (Appendix A). Chapter 2 concludes with suggested revisions to the lists of COC and COPC for the 300-FF-5 Operable Unit based on the data that have been collected and interpreted since the initial remedial investigation (DOE 1995) and the ROD (EPA 1996) and explanation of significant difference (EPA 2000).

Chapters 3 and 4 provide conceptual models for the uranium plume at the 300 Area and the tritium plume at the 618-11 burial ground sub-region, respectively. A summary of the data and interpretations used to develop these conceptual models are also provided. The conceptual models are intended to provide a technical basis to determine appropriate future actions within the operable unit.

Chapter 5 presents an analysis of each COC and COPC with respect to the monitoring objectives identified in the operation and maintenance plan (DOE 2002b). Section 5 also describes the EPA guidance for using monitored natural attenuation (MNA) as a remedy (EPA 1999), interprets the current 300-FF-5 Operable Unit remedy in the framework of this guidance, and assesses whether it is appropriate to consider MNA as a potential continuing remedy.

Appendix A contains uranium concentration trend charts for 300 Area wells. Appendix B is a copy of the Tri-Party Agreement Milestone M-016-83 agreed to by the U.S. Department of Energy (DOE) and EPA on March 11, 2005. Appendix C provides a description of the evolution of the regulatory framework for the 300-FF-5 Operable Unit. 


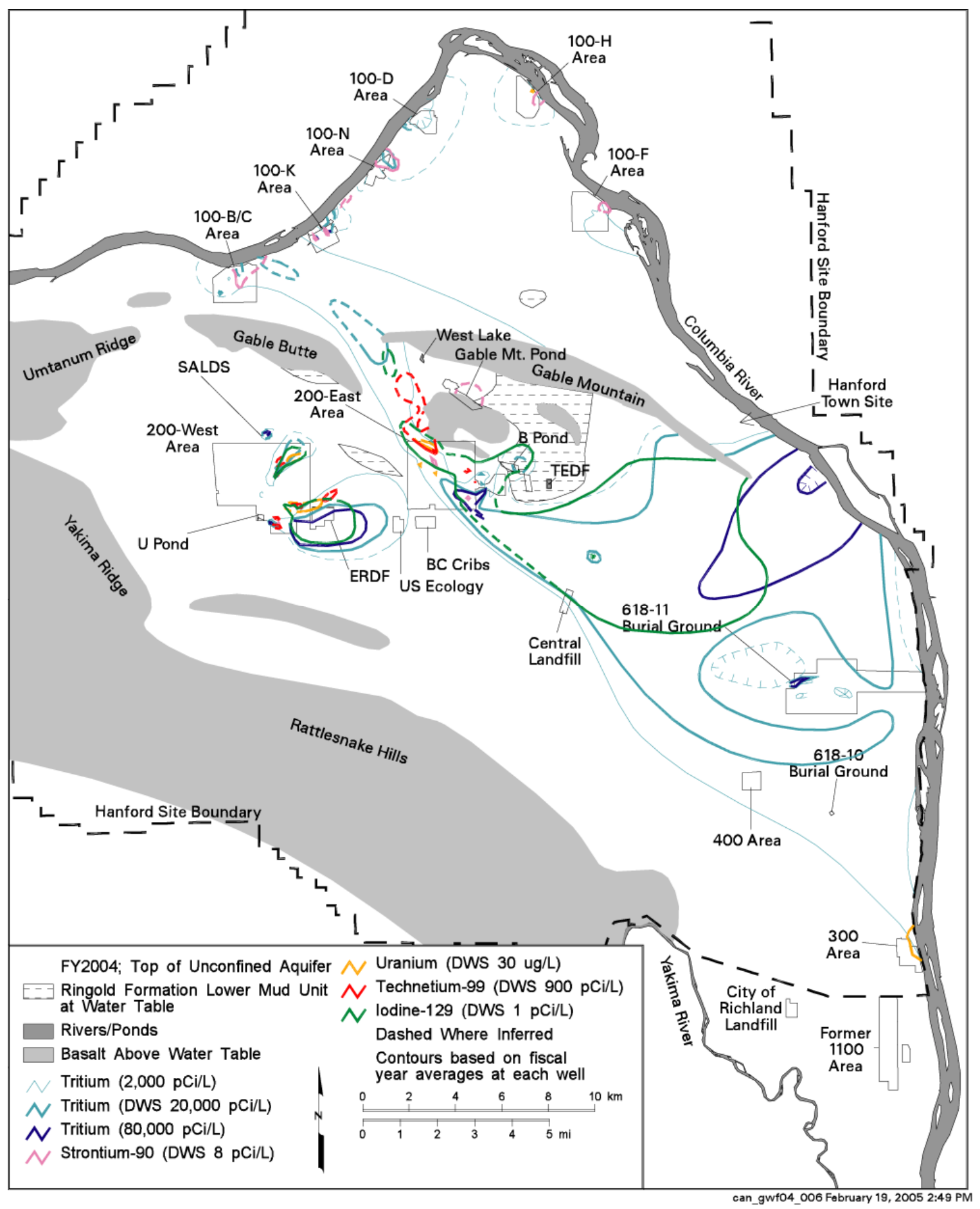

Figure 1.1. Map of the Hanford Site 


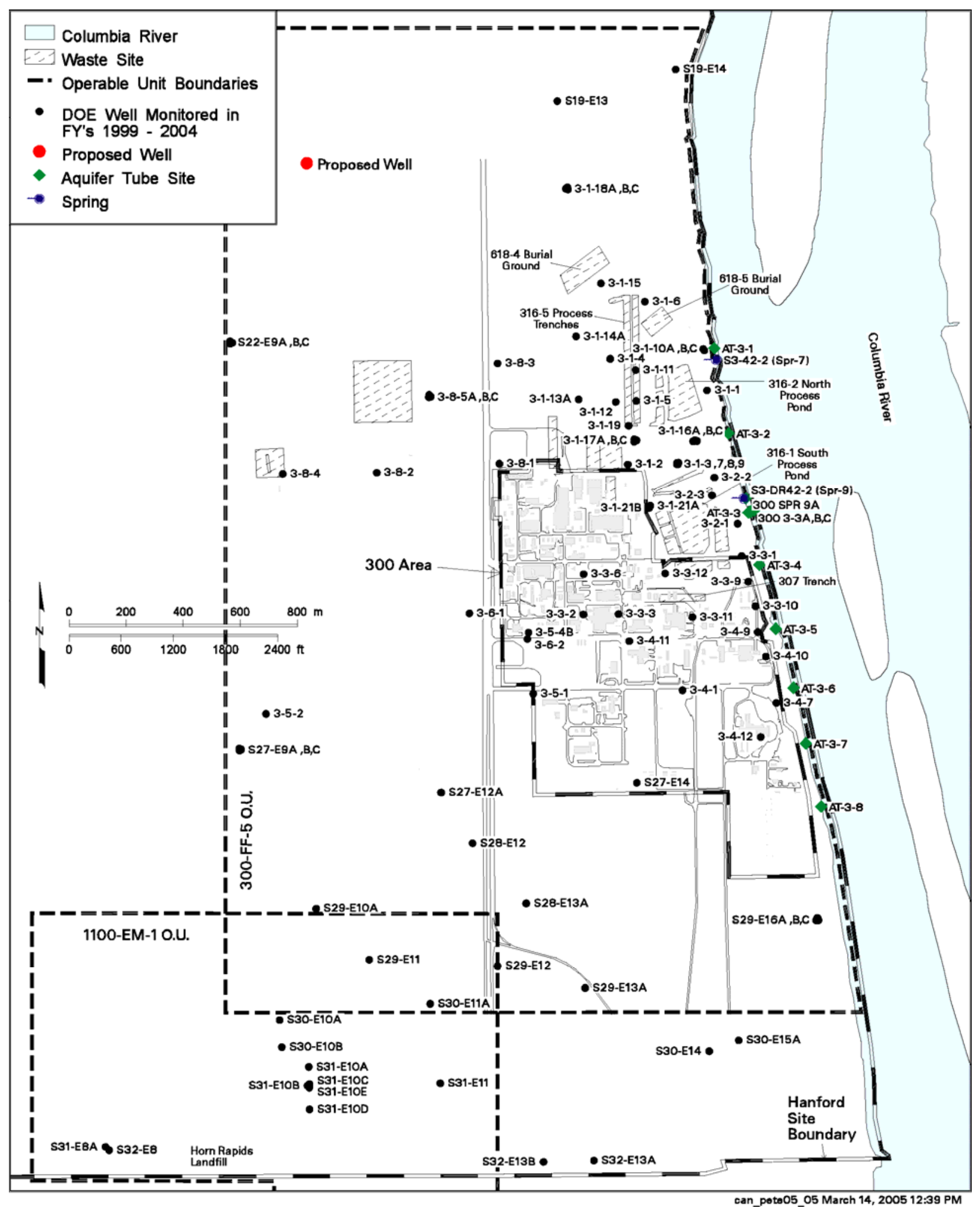

Figure 1.2. Locations of Monitoring Wells, Aquifer Tubes, Riverbank Springs, and Facilities at the 300 Area 

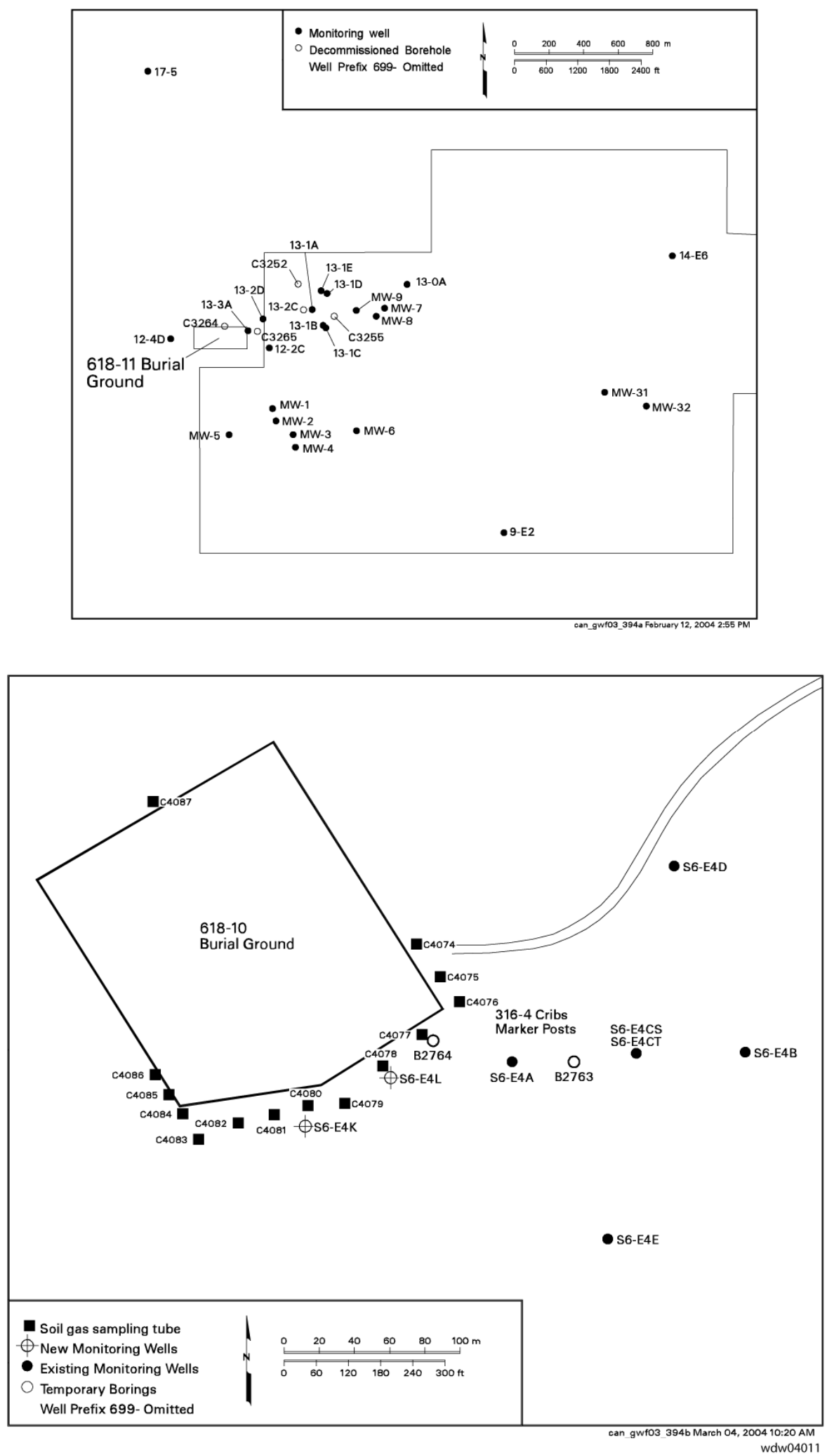

Figure 1.3. Locations of Monitoring Wells Near the 618-11 Burial Ground and 316-4 Cribs/ 618-10 Burial Ground Sub-Regions 


\subsection{Current Characteristics}

This section presents sampling and analysis results, and interpretations for groundwater constituents being monitored in the three sub-regions of the 300-FF-5 Operable Unit. The information supplements that routinely supplied via the annual groundwater project report (e.g., Lindberg and Peterson 2005). The data and interpretations include:

- Maximum observed concentrations by year for each well, for the period 1992 through 2004. Listing the maximum concentration is consistent with methods used earlier during the remedial investigation (DOE 1995) to characterize human and ecological health risks. The tables use different font characteristics to show results that are above or below the EPA maximum contaminant levels for drinking water supplies.

- Uranium plume maps are included that show conditions in the 300 Area for September 2001, June 2002, December 2002, June 2003, December 2003, June 2004, and December 2004. For each time period, estimates for the area of the plume, volume of contaminated groundwater, and mass of uranium in the plume are provided based on the contours and an assumed thickness for the contaminated layer.

- Uranium concentration trend charts for 300 Area wells that monitor the uppermost hydrologic unit (i.e., near the water table) are provided in Appendix A. These charts show (a) all results considered representative of aquifer conditions, (b) outlier or other non-representative results, and (c) values chosen as representative of concentrations for 2-year "windows." The latter values are used as input to the geostatistical analysis of the 300 Area uranium plume's characteristics (Chapter 3 of this report).

\subsection{Area Contaminants of Concern or Potential Concern}

The groundwater beneath the 300 Area has been contaminated by liquid effluent discharges to a variety of disposal sites during a period of operations that extends from the late 1940s through the mid1980s. Since the end of fuel fabrication activities, contaminated discharges have largely ceased, although discharges of uncontaminated effluent continued until 1994. Remedial actions have been completed that removed the structures and contaminated soil associated with most of these disposal sites. However, residual amounts of some contaminants remain in the underlying vadose zone, and their presence is indicated in groundwater monitoring results.

Some contaminants are currently present at concentrations that exceed the EPA's maximum contaminant level for drinking water supplies. The persistence of these contaminants, in the face of rapid flushing of the aquifer because of its high transmissivity characteristics, implies a continuing re-supply. Candidate non-point sources for uranium include releases from the (a) vadose zone beneath former waste sites, (b) widely distributed capillary fringe zone near the water table, and/or (c) aquifer solids.

COC in the groundwater at the 300 Area, as defined by the ROD (EPA 1996, pg. ii), are uranium, cis1,2-dichloroethene, and trichloroethene. Additional COPC, as identified during the remedial investigation (DOE 1995) or in RCRA corrective measures monitoring plans (Lindberg et al. 1995; 
Lindberg and Chou 2001), are tetrachloroethene, strontium-90, tritium, and nitrate. A detailed index map for 300 Area wells is provided in Figure 2.1.

\subsubsection{Uranium}

Table 2.1 shows the maximum yearly concentration of uranium at 300 Area monitoring wells during the period 1992 to 2004. Many results exceed the 30- $\mathrm{g} / \mathrm{L}$ drinking water standard (the proposed standard during the early 1990s was $20 \mu \mathrm{g} / \mathrm{L}$ ). The highest concentration observed during this period was $358 \mu \mathrm{g} / \mathrm{L}$ at well 399-2-2 in 1997.

An increase in uranium concentrations at many wells is readily apparent during the period 1995 through 1998. This time period correlates with major source excavation activities, cessation of clean water discharges to the 300 Area process trenches, unusually high water-table conditions during 1996 and 1997, and the apparent migration of a "pulse" of uranium introduced at the 300 Area process trenches during the early 1990s. Since 1998, there appears to be a general decline in uranium concentrations at most monitoring wells, although some wells show variable trends that reveal plume migration downgradient from known or suspected sources (e.g., 300 Area process trenches; 307 trench). During 2004, the highest uranium concentrations are observed at aquifer tube sites along the shoreline.

The geographic extent of the 300 Area uranium plume for the two seasons monitored (June and December) is shown in Figures 2.2 through 2.8, which cover the period 2001 to 2004. The overall extent of uranium contamination, as illustrated by the $10-\mu \mathrm{g} / \mathrm{L}$ contour line, remains fairly constant for this time period. ${ }^{1}$ The maximum concentration areas outlined by the contours appear to gradually decrease with time. Two areas of relatively high concentrations occasionally appear during the June seasonal high water-table period: one near the south end of the 300 Area process trenches, and a second to the east of the 307 process trenches (Figures 2.3, 2.5, and 2.7). The current explanation for these features is that the lower vadose zone at each of these locations is contaminated with uranium, which is remobilized during periods of high water-table conditions. Source removal actions have been completed for the 300 Area process trenches, and will be addressed at the 307 trench following decontamination and decommissioning activities of surface structures.

Source removal actions at the 618-5 burial ground in October 2002 are believed to have remobilized uranium in the vadose zone at that waste site. The remobilized uranium migrated downgradient with groundwater flow to well 399-1-10A by December 2002, causing an increasing trend at the well (Figure 2.4). The pulse of uranium created in October 2002 appears to be dissipating (Figures 2.5 through 2.8; trend chart for well 399-1-10A in Appendix A). Based on the timing of excavation activities and the arrival at well 399-1-10A and the distance to well 399-1-10A, a plume migration rate on the order of meters per day is estimated.

Estimates for the areal extent, contaminated volume, and dissolved mass for the 300 Area uranium plume are listed in Table 2.2. The estimates were derived from the six seasonal plume maps shown in Figures 2.2 through 2.8. A summary of values for these plume parameters is presented in Table 2.3. The areal extent of the plume at levels greater than the drinking water standard (30 $\mu \mathrm{g} / \mathrm{L})$ ranges between 0.36 and 0.43 square kilometers ( 0.14 to 0.17 square miles). The volume of groundwater contaminated

\footnotetext{
${ }^{1}$ Natural background for uranium in this area falls in the range 5 to $8 \mu \mathrm{g} / \mathrm{L}$.
} 
above the drinking water standard ranges between 262,215 and 367,577 cubic meters (992 to 1.391 million gallons). The mass of dissolved uranium in the greater than $30-\mu \mathrm{g} / \mathrm{L}$ plume ranges from 15.7 to 30.4 kilograms (35 to 67 pounds). ${ }^{2}$

The differences in plume parameters caused by seasonal conditions are also revealed in Table 2.3, where June area and mass values tend to be somewhat smaller than December values. This is generally true for the six seasonal periods presented, with the exception of December 2003, which shows anomalously low values for all parameters. The lower mass values for June are believed to reflect dilution of groundwater (i.e., reduced concentrations) at shoreline wells because of infiltrating river water during the high river stage in June. The highest mass values occurred during December 2002 and may reflect the pulse of uranium detected at well 399-1-10A (see trend chart in Appendix A), which is a short-term consequence of excavation activities at the 618-5 burial ground.

Using the observational data for uranium concentrations for December 2003, estimates for the mass of uranium in the mapped plume (i.e., $>10 \mu \mathrm{g} / \mathrm{L}$ ) were also made using EarthVision ${ }^{\mathrm{TM}}$ software. The effective porosity was assumed to be $25 \%$. The total mass in the plume was calculated at 39.9 kilograms (87.9 pounds), which is higher than the estimates summarized in Table 2.3, but still within the range of expected values, and consistent with the mass estimates that resulted from the geostatistical analysis of plume parameters (see Chapter 3).

A water supply well (399-4-12) operates in the south portion of the 300 Area to supply water for the aquariums in the 331 Building (see Figure 2.1 for location, and trend chart in Appendix A for uranium concentrations). This well has a pumping rate that typically varies in the range of 757 to 2,271 liters (200 to 600 gallons) per minute and has been in operation since approximately 1982. Assuming an average pumping rate of 1,234.9 liters (350 gallons) per minute for 22 years, and an average uranium concentration of $30 \mu \mathrm{g} / \mathrm{L}$ during that period, approximately 460 kilograms (1,014 pounds) of uranium would have passed through this well. The aquarium water is typically made up of a mixture of water from the well, and water drawn from the river. The effluent from the aquariums was initially returned to the river via an outfall pipe as a permitted discharge. Currently, the effluent no longer requires a permit and flows over the riverbank as a small stream. The stream is monitored by the Surface Environmental Surveillance Project.

During February 2004, eight sites along the 300 Area shoreline were equipped with aquifer tubes, with each site having up to three tubes at various depths. The three depths are intended to represent conditions near the water table; as deep as logistically possible using the hand-held installation equipment; and a depth mid-way between the other two. The first sampling of these tubes occurred during March 2004; the results for uranium analyses are shown in Figure 2.9.

The highest uranium concentrations occur at sites AT-3-3 (195 $\mu \mathrm{g} / \mathrm{L})$ and AT-3-4 (241 $\mu \mathrm{g} / \mathrm{L})$, which are located adjacent to the shoreline where relatively high uranium concentrations are found in groundwater. The specific conductance for these samples indicated that they are predominantly groundwater, i.e., there has been minimal dilution by infiltrating river water. Uranium in groundwater along this segment of shoreline is likely to have originated in the vicinity of the 300 Area process trenches. Uranium concentrations in tubes at the north end of the 300 Area also show results that are consistent

\footnotetext{
${ }^{2}$ Assumptions: Contaminated layer thickness of 3 meters (9.8 feet) for December and 3.3 meters (10.8 feet) for June; effective porosity of $27 \%$; and mid-point concentrations between contour intervals for each segment.
} 
with adjacent groundwater. Concentrations ranging from 87 to $100 \mu \mathrm{g} / \mathrm{L}$ at site AT-3-1 (Figure 2.9) and probably reflect uranium whose origin is the 618-5 excavation site (see Figure 2.4).

At tube sites adjacent to the second portion of the 300 Area uranium plume that shows relatively elevated concentrations, the March 2004 tube results appear to be consistent with adjacent groundwater results, although concentrations are not as high as along the segment just upstream. The origin for uranium along this segment of shoreline is believed to be located in the vicinity of the 307 trench. 
Table 2.1. Maximum Uranium Concentrations ( $\mu \mathrm{g} / \mathrm{L})$ at 300 Area Wells

\begin{tabular}{|c|c|c|c|c|c|c|c|c|c|c|c|c|c|c|}
\hline Well Name & Unit & 1992 & 1993 & 1994 & 1995 & 1996 & 1997 & 1998 & 1999 & 2000 & 2001 & 2002 & 2003 & 2004 \\
\hline \multicolumn{15}{|c|}{ Wells that monitor the top of the unconfined aquifer } \\
\hline $399-1-1$ & TU & & & & & & & & & & & 62 & 53 & 66 \\
\hline $399-1-2$ & TU & & 21 & 28 & & 52 & 35 & 10 & 16 & 7 & 6 & 47 & 10 & 13 \\
\hline $399-1-3$ & TU & & & & 53 & & & 134 & 153 & 100 & 65 & & & \\
\hline 399-1-4 & $\mathrm{TU}$ & & 5 & & 14 & & & & & & & & & \\
\hline 399-1-5 & TU & ND & 110 & 120 & & 105 & 80 & 51 & 48 & (a) & (a) & (a) & (a) & (a) \\
\hline 399-1-6 & TU & 23 & 7 & 9 & 9 & 13 & 11 & 8 & 9 & & & & 9 & 7 \\
\hline 399-1-7 & $\mathrm{TU}$ & 160 & 110 & 131 & & 248 & 329 & 132 & 153 & 95 & 72 & 90 & 80 & 67 \\
\hline 399-1-10A & TU & 152 & 110 & 71 & 75 & 85 & 144 & 75 & 61 & 53 & 43 & 235 & 178 & 67 \\
\hline 399-1-11 & $\mathrm{TU}$ & 48 & 21 & 31 & 33 & 102 & 47 & & & & 9 & 37 & 28 & 16 \\
\hline 399-1-12 & $\mathrm{TU}$ & 83 & 44 & 50 & 60 & 83 & 53 & 22 & 23 & 16 & 17 & 40 & 22 & 21 \\
\hline 399-1-13A & TU & 6 & 6 & & 7 & 14 & & & & & & & & \\
\hline $399-1-14 \mathrm{~A}$ & TU & 6 & 6 & 7 & 8 & 20 & 13 & 6 & 8 & 6 & 6 & & & \\
\hline 399-1-15 & $\mathrm{TU}$ & 5 & 6 & 4 & & 7 & & & & & & 7 & 7 & 6 \\
\hline 399-1-16A & TU & 156 & 145 & 87 & 165 & 123 & 137 & 124 & 115 & 136 & 100 & 94 & 86 & 88 \\
\hline 399-1-17A & TU & 111 & 43 & 71 & 247 & 300 & 313 & 248 & 166 & 126 & 64 & 70 & 70 & 56 \\
\hline 399-1-18A & TU & 5 & 5 & 6 & 5 & 6 & 7 & 6 & 6 & 6 & 6 & & 7 & 7 \\
\hline 399-1-19 & $\mathrm{TU}$ & & & ND & 198 & 271 & (a) & (a) & (a) & (a) & (a) & (a) & (a) & (a) \\
\hline 399-1-21A & $\mathrm{TU}$ & 82 & 46 & 23 & 24 & 63 & 101 & 36 & 32 & 18 & 12 & 47 & 22 & 31 \\
\hline 399-2-1 & $\mathrm{TU}$ & 48 & 46 & 58 & 159 & 196 & 230 & 252 & 232 & & 169 & 149 & 79 & 61 \\
\hline 399-2-2 & $\mathrm{TU}$ & ND & 110 & 150 & 160 & 262 & 358 & 225 & 322 & 205 & 144 & 137 & 66 & 86 \\
\hline 399-2-3 & $\mathrm{TU}$ & 42 & $\mathrm{ND}$ & 118 & 147 & 68 & & & & & & & & \\
\hline 399-3-1 & $\mathrm{TU}$ & & & 53 & 81 & 56 & 189 & 219 & 285 & 234 & 198 & & & \\
\hline 399-3-2 & $\mathrm{TU}$ & 15 & 26 & 22 & 25 & & & & & & & & & \\
\hline 399-3-3 & $\mathrm{TU}$ & 10 & & 16 & & 18 & 27 & & 17 & 12 & & & & \\
\hline 399-3-6 & TU & & & 17 & 33 & 46 & 34 & 20 & 12 & 11 & & 22 & 14 & 14 \\
\hline 399-3-9 & $\mathrm{TU}$ & 27 & & 22 & & & & & & & & & & \\
\hline 399-3-10 & $\mathrm{TU}$ & 14 & 26 & 20 & 63 & 36 & 74 & 92 & 84 & 140 & 181 & 141 & 127 & 92 \\
\hline \begin{tabular}{|l|}
$399-3-11$ \\
\end{tabular} & TU & 24 & 38 & 97 & 130 & & 66 & 35 & 48 & 28 & 23 & 85 & 42 & 107 \\
\hline 399-3-12 & $\mathrm{TU}$ & 32 & 37 & 39 & & 76 & 78 & 29 & 37 & & 15 & 78 & 33 & 20 \\
\hline 399-4-1 & $\mathrm{TU}$ & 16 & 14 & 17 & 20 & 37 & 54 & 26 & 20 & 16 & 18 & 24 & 20 & 16 \\
\hline 399-4-7 & TU & 59 & 51 & 53 & 43 & 29 & 69 & 68 & 73 & 73 & 63 & & & \\
\hline 399-4-9 & $\mathrm{TU}$ & 50 & ND & 39 & & 74 & 128 & 128 & 163 & 152 & 110 & 46 & 104 & 83 \\
\hline $399-4-10$ & TU & 56 & 70 & 55 & 38 & & & & & & 94 & 94 & & 91 \\
\hline 399-4-11 & TU & 13 & 17 & & 24 & 40 & 51 & 23 & 20 & 16 & 17 & & & \\
\hline 399-4-12 & $\mathrm{TU}$ & 25 & 25 & 23 & 21 & 40 & 43 & 32 & 37 & 22 & 21 & 24 & 22 & 22 \\
\hline 399-5-1 & $\mathrm{TU}$ & 7 & 6 & 5 & 5 & 12 & 10 & 7 & 9 & 7 & 7 & & & \\
\hline 399-6-1 & $\mathrm{TU}$ & 10 & 9 & & & & & & & & & & & \\
\hline 399-8-1 & $\mathrm{TU}$ & 5 & 5 & 5 & 5 & 12 & 17 & 8 & 5 & 5 & 5 & & & \\
\hline 399-8-3 & $\mathrm{TU}$ & 5 & 5 & & & 14 & & & & & & & & \\
\hline 399-8-4 & $\mathrm{TU}$ & 3 & & & & & & & & & & & & \\
\hline 399-8-5A & $\mathrm{TU}$ & 11 & 11 & 7 & & 4 & 8 & 19 & 7 & 10 & 8 & 5 & 26 & 7 \\
\hline \begin{tabular}{|l|} 
699-S19-E13 \\
\end{tabular} & $\mathrm{TU}$ & 4 & 5 & 5 & 5 & & & & & & & & & \\
\hline \begin{tabular}{|l|} 
699-S19-E14 \\
\end{tabular} & TU & 6 & 4 & & & & & & & & & & & \\
\hline 699-S27-E12A & $\mathrm{TU}$ & & & & & & & 7 & 8 & 9 & & & & \\
\hline 699-S27-E14 & $\mathrm{TU}$ & & & & & 7 & 8 & 8 & 7 & 7 & 8 & 9 & 7 & 8 \\
\hline 699-S29-E12 & $\mathrm{TU}$ & & & 4 & & & & & 6 & & & & & \\
\hline \begin{tabular}{|l|}
$699-S 29-E 16 A$ \\
\end{tabular} & TU & 2 & 1 & 3 & 2 & 3 & 5 & 4 & & 5 & 4 & 3 & 4 & 4 \\
\hline 699-S30-E15A & $\mathrm{TU}$ & 2 & 3 & 2 & 2 & & & & 4 & 4 & 4 & 4 & 4 & 4 \\
\hline
\end{tabular}


Table 2.1. (contd)

\begin{tabular}{|c|c|c|c|c|c|c|c|c|c|c|c|c|c|c|}
\hline Well Name & Unit & 1992 & 1993 & 1994 & 1995 & 1996 & 1997 & 1998 & 1999 & 2000 & 2001 & 2002 & 2003 & 2004 \\
\hline \multicolumn{15}{|c|}{ Aquifer tubes at the shoreline that monitor the top of the unconfined aquifer } \\
\hline 300-3-3A-410cm & TU-S & & & & & & & & & & & & & 195 \\
\hline 300-3-3B-376cm & TU-S & & & & & & & & & & & & & 192 \\
\hline 300-SPR9A-19cm & TU-S & & & & & & & & & & & & & 107 \\
\hline 300-SPR9A-86cm & TU-S & & & & & & & & & & & & & 138 \\
\hline AT-3-1-D(1) & TU-S & & & & & & & & & & & & & 93 \\
\hline AT-3-1-M & TU-S & & & & & & & & & & & & & 100 \\
\hline AT-3-1-S & TU-S & & & & & & & & & & & & & 87 \\
\hline AT-3-2-M & TU-S & & & & & & & & & & & & & 88 \\
\hline AT-3-3-D & TU-S & & & & & & & & & & & & & 10 \\
\hline AT-3-3-M & TU-S & & & & & & & & & & & & & 183 \\
\hline AT-3-3-S & TU-S & & & & & & & & & & & & & 195 \\
\hline AT-3-4-S & TU-S & & & & & & & & & & & & & 241 \\
\hline AT-3-5-S & TU-S & & & & & & & & & & & & & 52 \\
\hline AT-3-6-S & TU-S & & & & & & & & & & & & & 85 \\
\hline AT-3-7-M & TU-S & & & & & & & & & & & & & 18 \\
\hline AT-3-8-S & TU-S & & & & & & & & & & & & & 18 \\
\hline \multicolumn{15}{|c|}{ Wells that monitor the bottom of the unconfined aquifer } \\
\hline 399-1-8 & $\mathrm{BU}$ & 110 & $\mathbf{0}$ & $\mathbf{0}$ & & & & & & & 3 & 17 & $41^{(\mathrm{b})}$ & 14 \\
\hline 399-1-10B & $\mathrm{BU}$ & $\mathbf{0}$ & ND & $\mathbf{0}$ & $\mathbf{0}$ & & $\mathbf{0}$ & $\mathbf{0}$ & $\mathbf{0}$ & $\mathbf{0}$ & $\mathbf{0}$ & $\mathbf{0}$ & 3 & 0.5 \\
\hline 399-1-13B & $\mathrm{BU}$ & $\mathbf{0}$ & $\mathbf{0}$ & $\mathbf{0}$ & ND & ND & & & & & & & & \\
\hline 399-1-14B & $\mathrm{BU}$ & $\mathbf{0}$ & $\mathbf{0}$ & ND & 1 & ND & $\mathbf{0}$ & & & & & & & \\
\hline 399-1-16B & $\mathrm{BU}$ & 5 & 5 & 6 & 9 & 3 & 14 & 15 & 14 & 14 & 15 & 14 & 14 & 14 \\
\hline 399-1-17B & $\mathrm{BU}$ & $\mathbf{0}$ & ND & $\mathbf{0}$ & ND & $\mathbf{0}$ & $\mathbf{0}$ & $\mathbf{0}$ & ND & 1 & 1 & 1 & 3 & 0.4 \\
\hline 399-1-18B & $\mathrm{BU}$ & & & & & $\mathbf{0}$ & $\mathbf{0}$ & $\mathbf{0}$ & ND & $\mathbf{0}$ & $\mathbf{0}$ & & $\mathbf{0}$ & 0.2 \\
\hline 399-1-21B & $\mathrm{BU}$ & $\mathbf{0}$ & $\mathbf{0}$ & $\mathbf{0}$ & & $\mathbf{0}$ & & & & & $\mathbf{0}$ & 1 & $\mathbf{0}$ & $49^{(\mathrm{b})}$ \\
\hline 699-S29-E16B & $\mathrm{BU}$ & $\mathbf{0}$ & $\mathbf{0}$ & $\mathbf{0}$ & $\mathbf{0}$ & & & & & & & & & \\
\hline \multicolumn{15}{|c|}{ Wells that monitor the uppermost confined aquifer } \\
\hline 399-1-9 & $\mathrm{C}$ & ND & $\mathbf{0}$ & 1 & & & & & & & & & & \\
\hline $399-1-16 C$ & $\mathrm{C}$ & ND & 6 & $\mathbf{0}$ & $\mathbf{0}$ & ND & & & & & & & & \\
\hline 399-8-5B & $\mathrm{C}$ & $\mathbf{0}$ & $\mathbf{0}$ & $\mathbf{0}$ & $\mathbf{0}$ & & & & & & & & & \\
\hline $399-8-5 \mathrm{C}$ & $\mathrm{C}$ & ND & $\mathbf{0}$ & $\mathbf{0}$ & ND & & & & & & & & & \\
\hline 699-S29-E16C & $\mathrm{C}$ & ND & $\mathbf{0}$ & $\mathbf{0}$ & $\mathbf{0}$ & & & & & & & & & \\
\hline $\begin{array}{l}\text { (a) Well out-of-s } \\
\text { (b) Values are un } \\
\text { Note: Maximum v } \\
\text { Blanks indicate "nc } \\
\text { BU = Bottom of } \\
\mathrm{C}=\text { First conf } \\
\text { EPA }=\text { U.S. Envi } \\
\text { ND = Not detec } \\
\text { TU = Top of un } \\
\text { TU-S }=\text { Top of un }\end{array}$ & $\begin{array}{l}\text { ice. } \\
\text { review } \\
\text { es for u } \\
\text { sults." } \\
\text { confine } \\
\text { d aquife } \\
\text { mental } \\
\text { fined ac } \\
\text { fined ac }\end{array}$ & 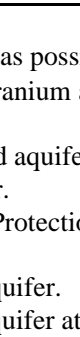 & $\begin{array}{l}\text { ble rep } \\
\text { t } 300 \mathrm{~A} \\
\text { shoreli }\end{array}$ & $\begin{array}{l}\text { rting en } \\
\text { rea wel }\end{array}$ & $\begin{array}{l}\text { ors. } \\
\text { s. Valu }\end{array}$ & in $b$ & in sh & ded cel & are be & w the & EPA & king & ter st & $\mathrm{d}(30 \mu \mathrm{g} / \mathrm{L})$ \\
\hline
\end{tabular}




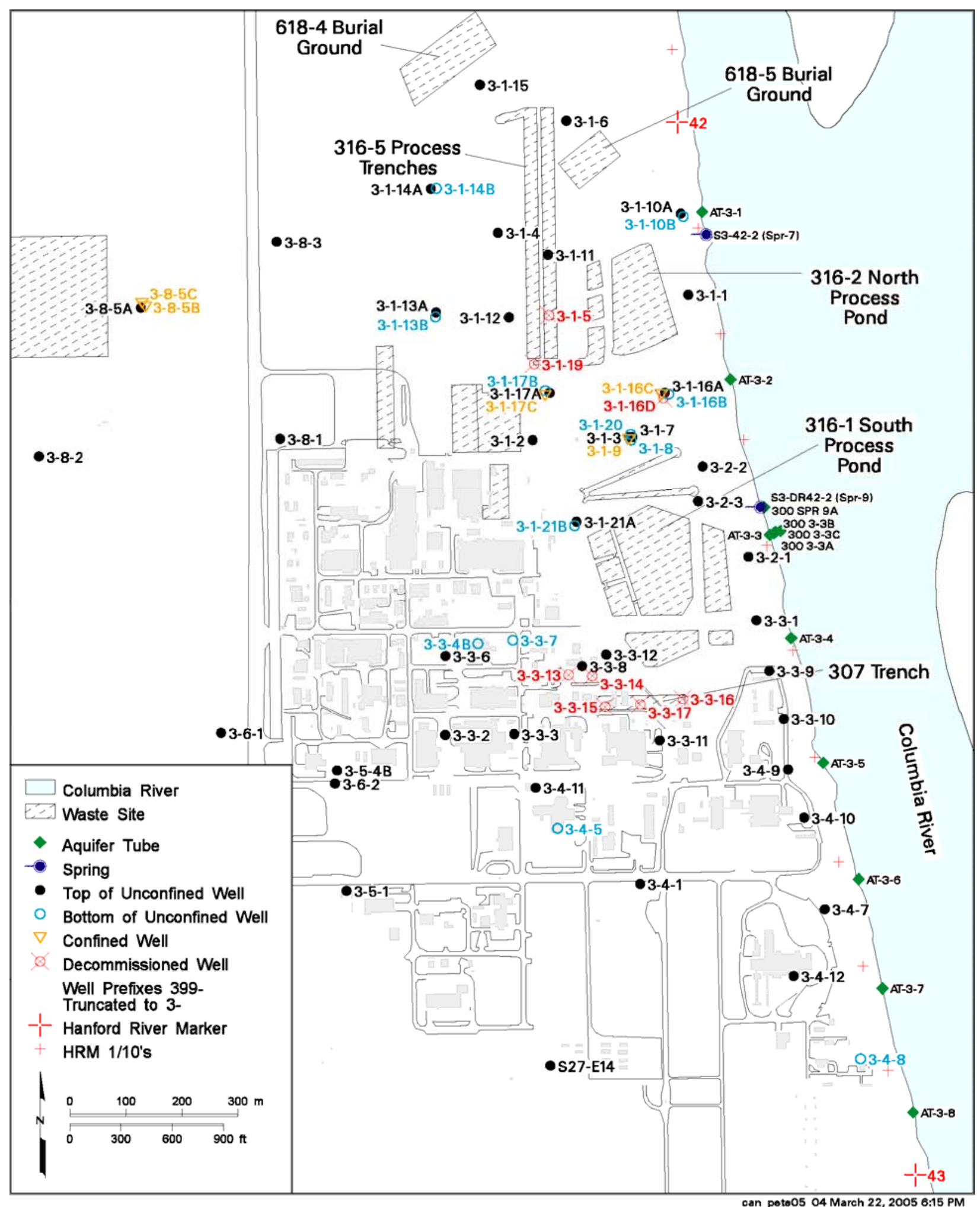

Figure 2.1. Location of 300 Area Wells 


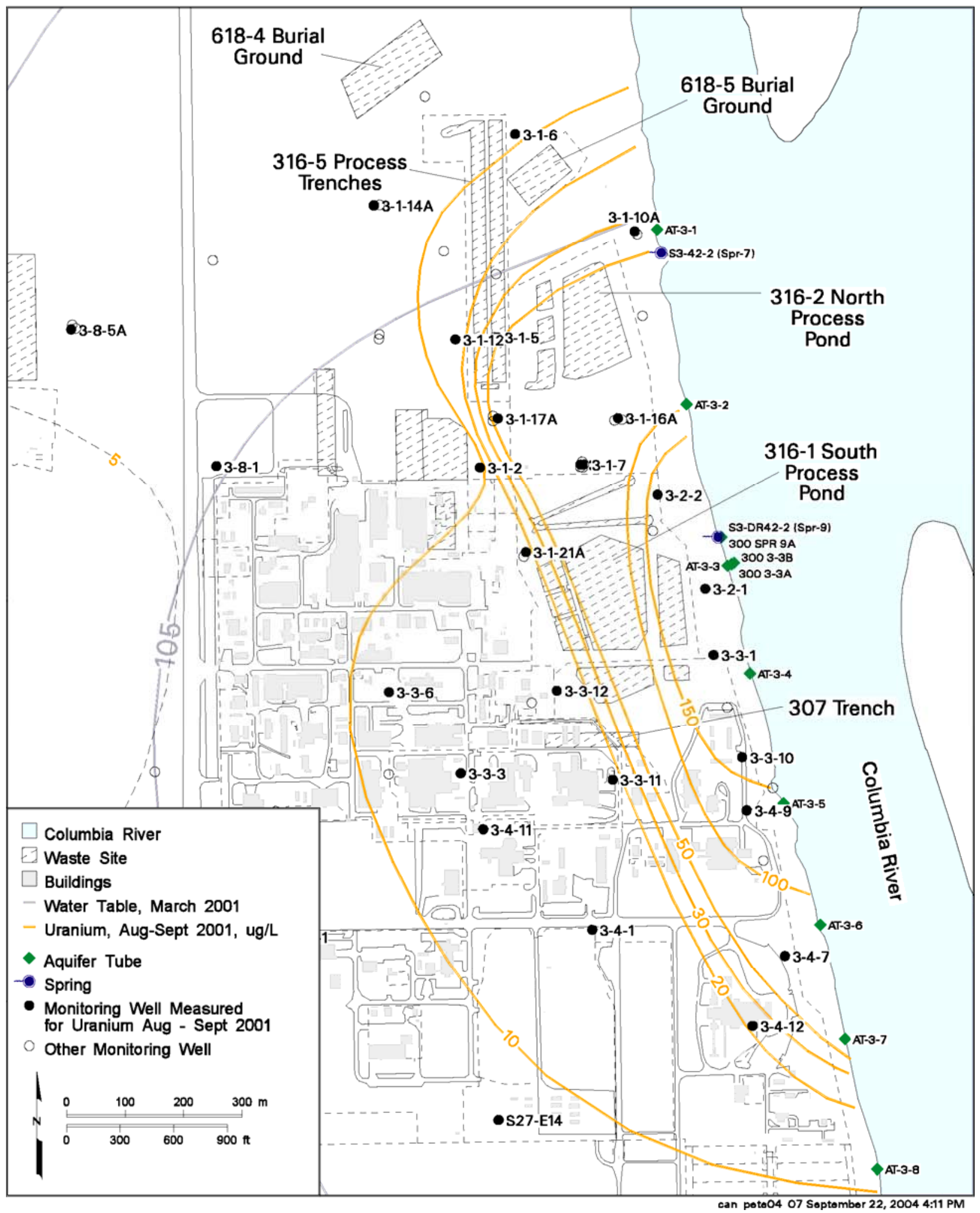

Figure 2.2. 300 Area Uranium Plume, August/September 2001 Conditions 


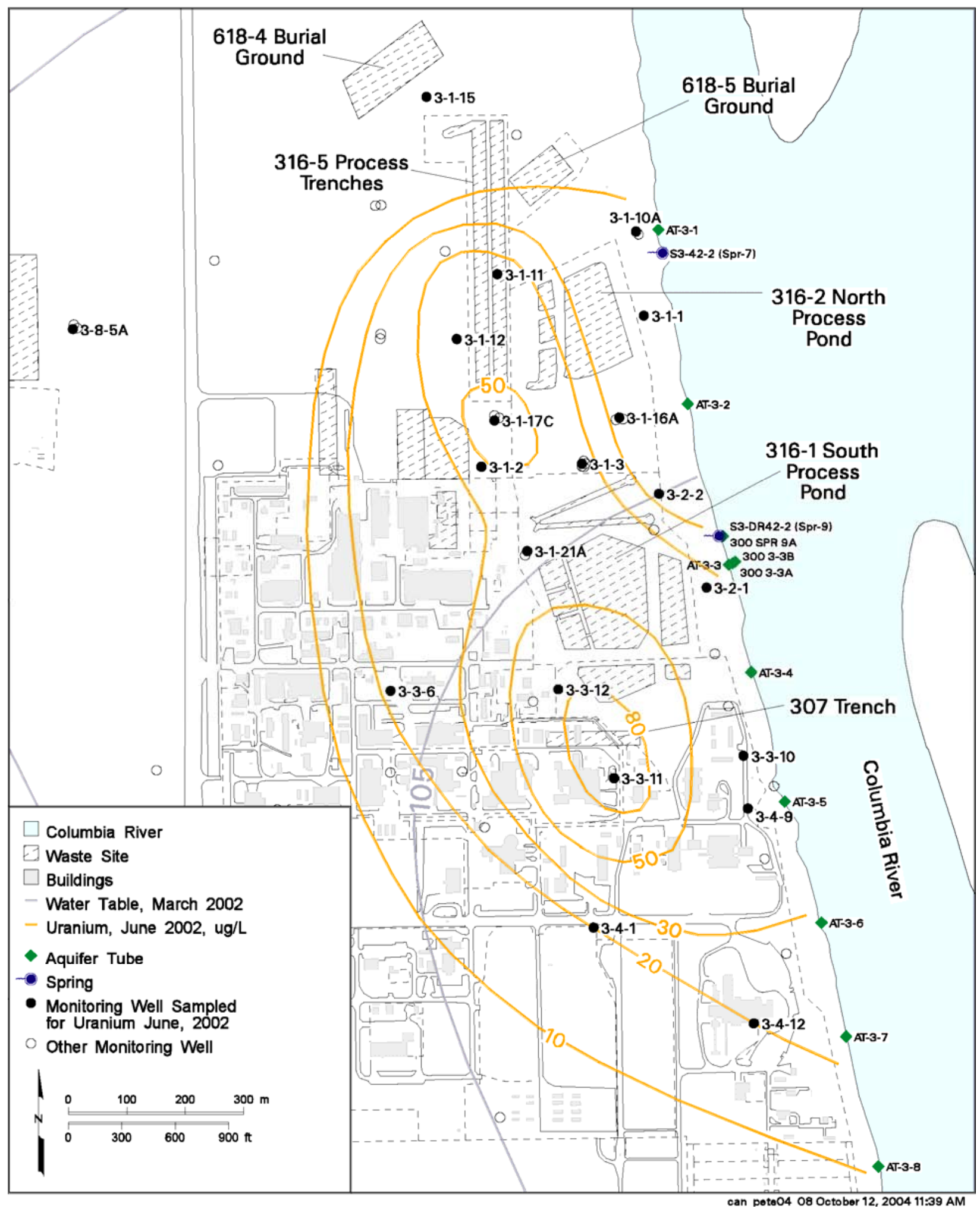

Figure 2.3. 300 Area Uranium Plume, June 2002 Conditions 


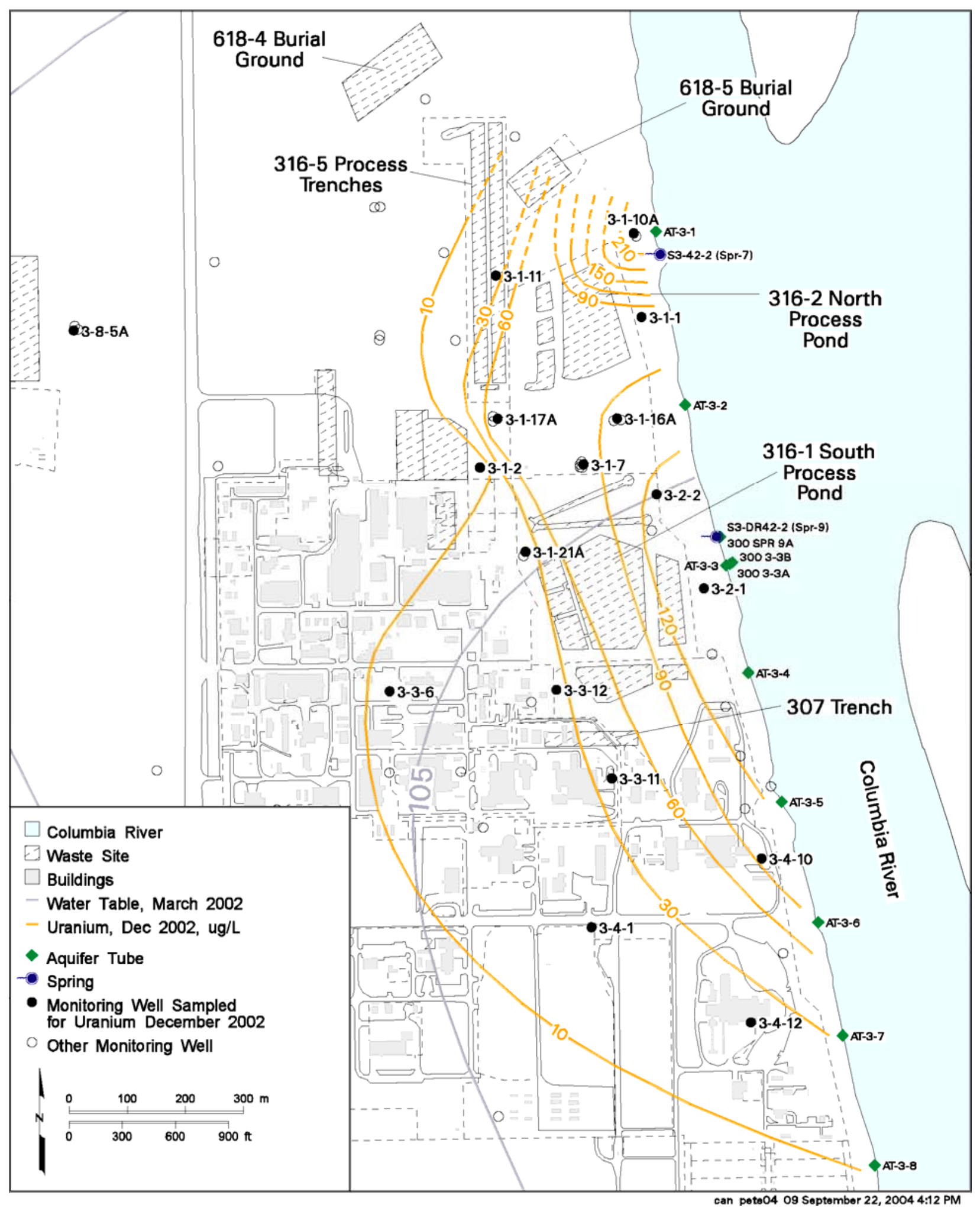

Figure 2.4. 300 Area Uranium Plume, December 2002 Conditions 


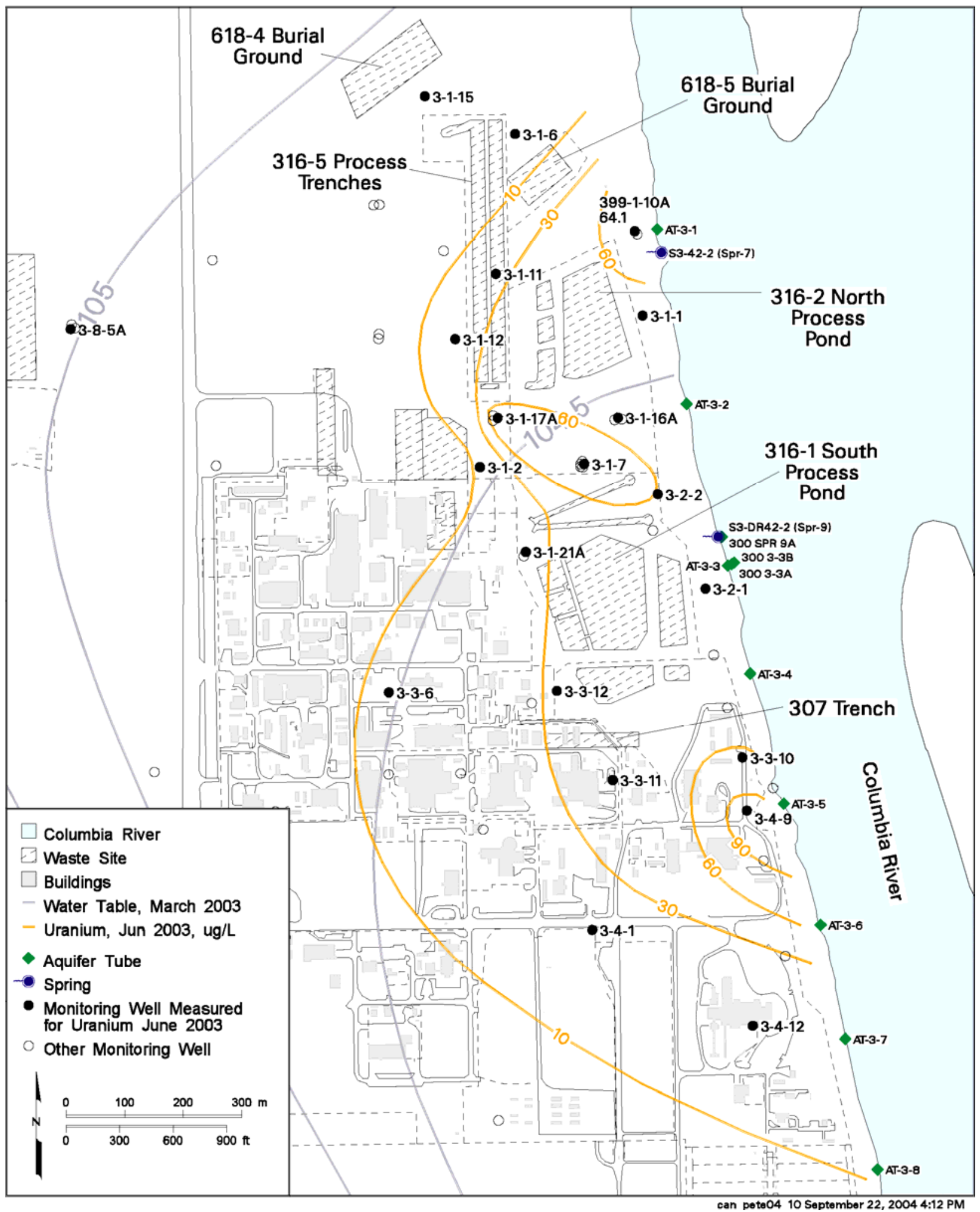

Figure 2.5. 300 Area Uranium Plume, June 2003 Conditions 


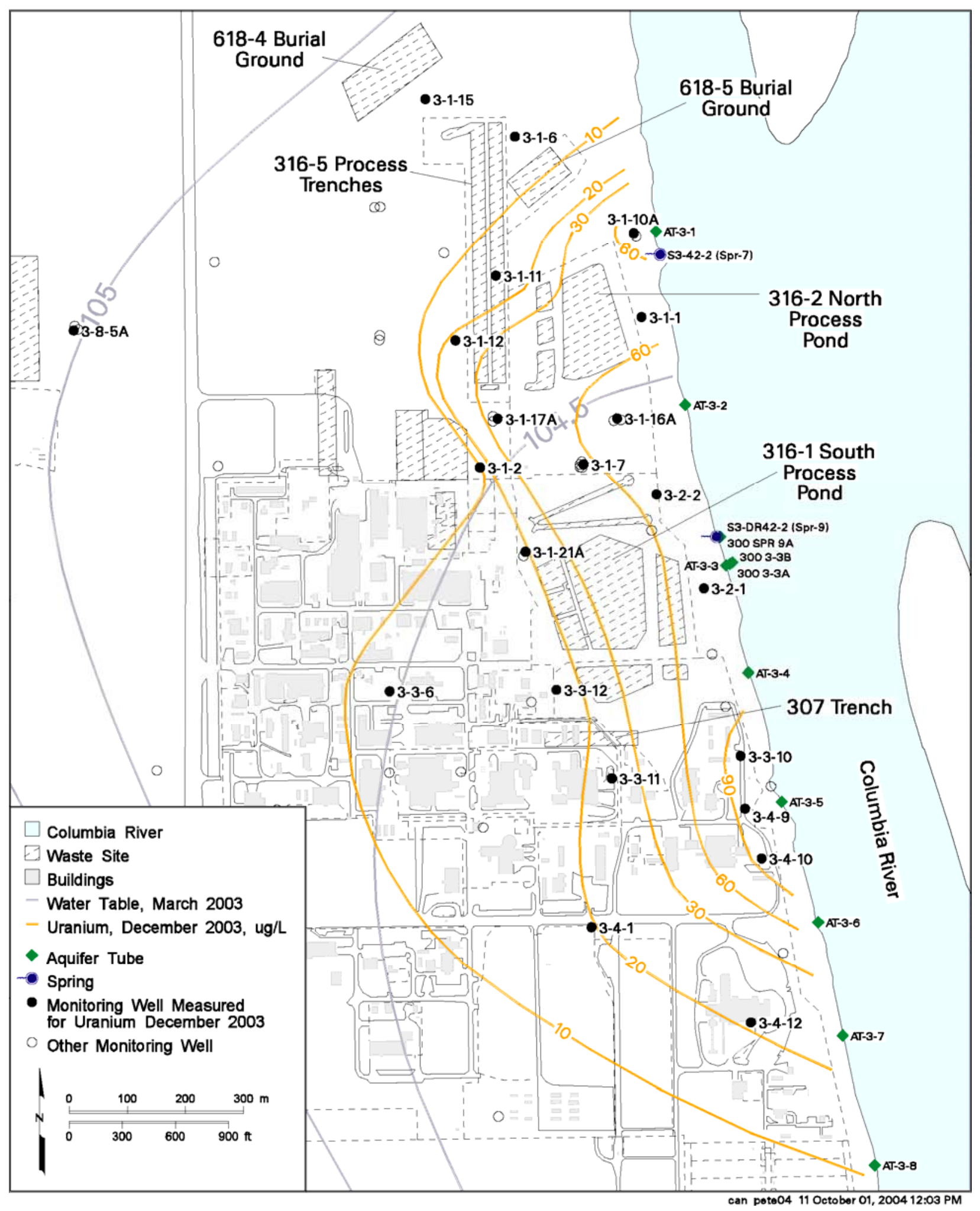

Figure 2.6. 300 Area Uranium Plume, December 2003 Conditions 


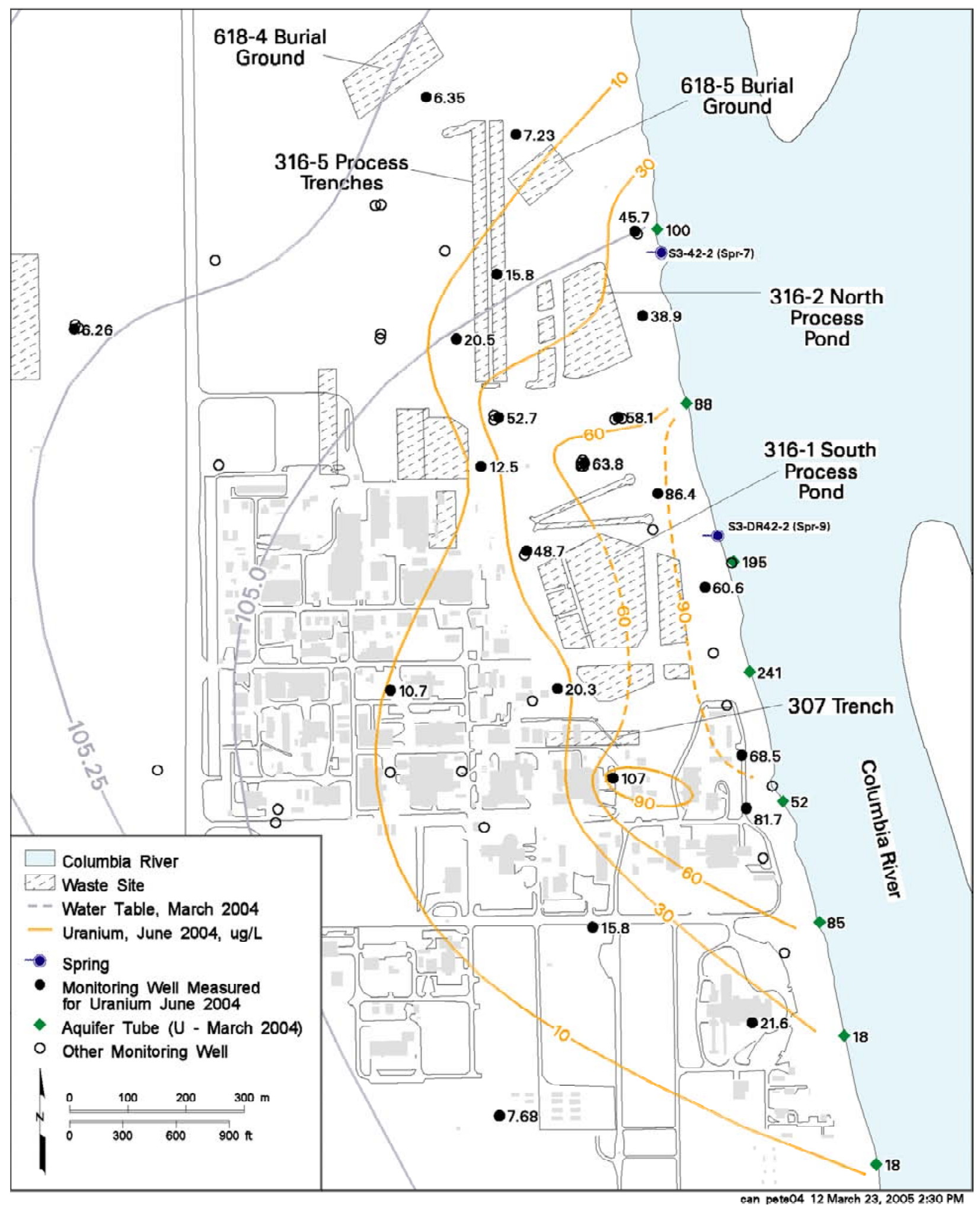

Figure 2.7. 300 Area Uranium Plume, June 2004 Conditions 


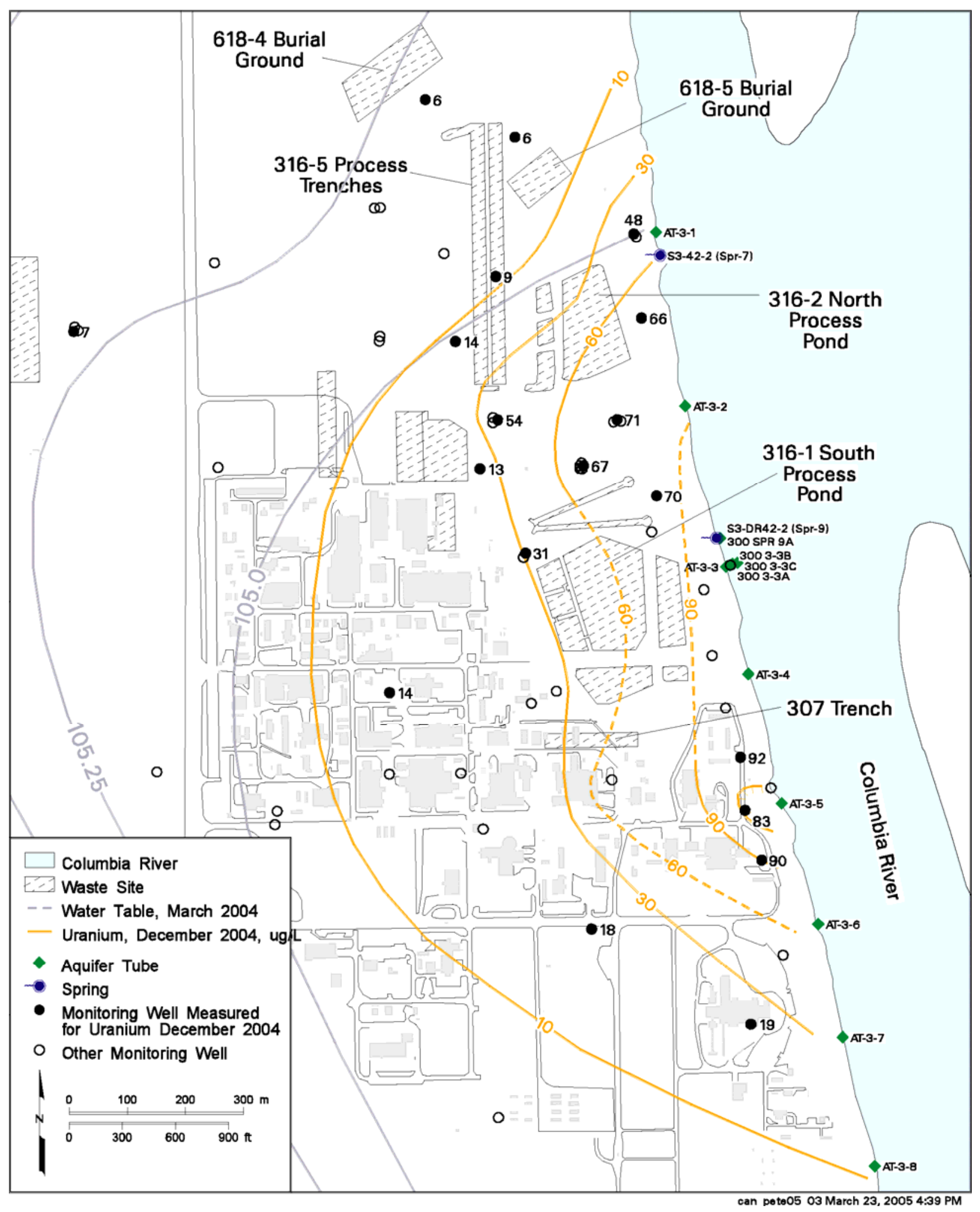

Figure 2.8. 300 Area Uranium, December 2004 


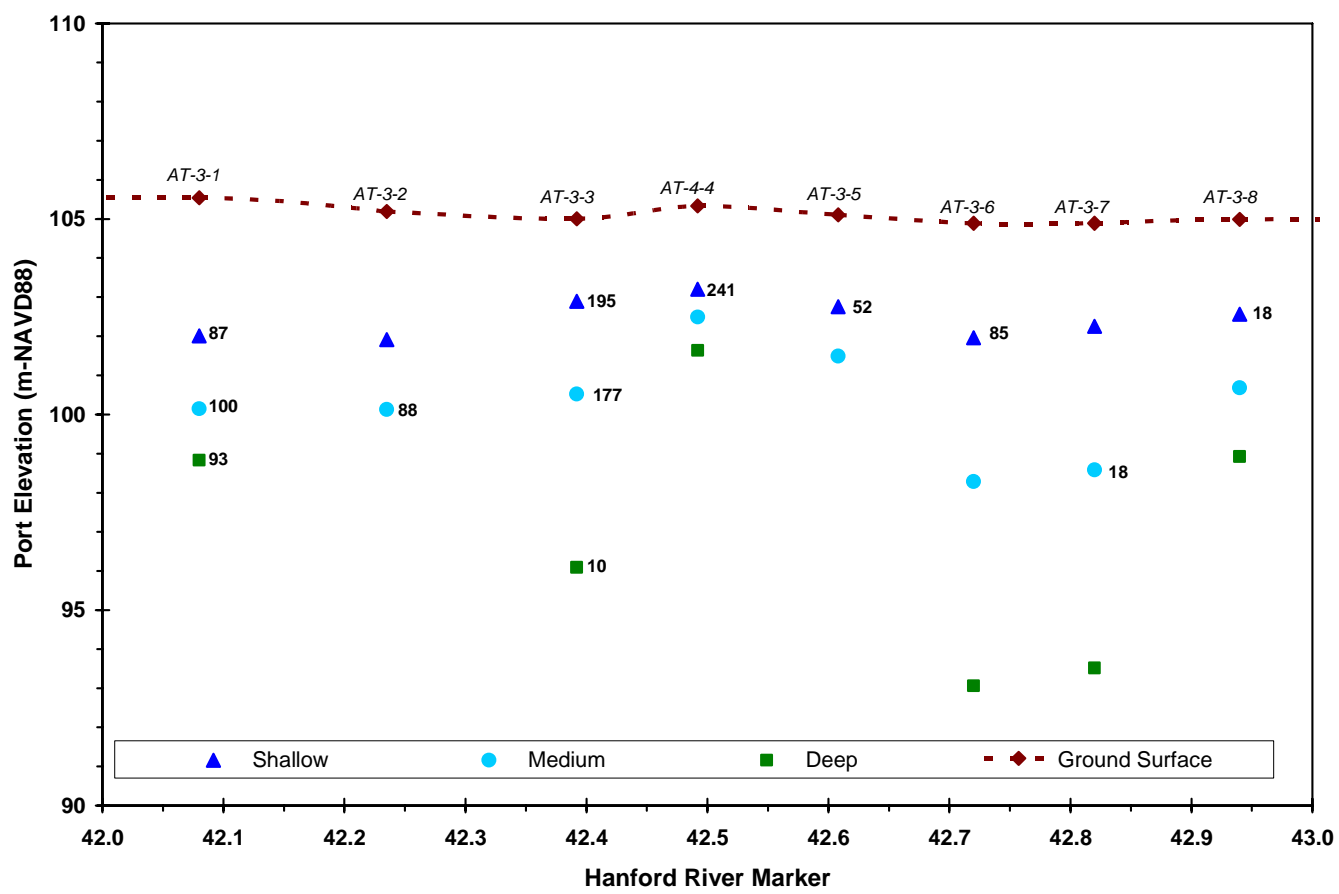

Figure 2.9. Uranium Concentrations at Aquifer Tubes Along 300 Area Shoreline (March 2004 Results) 
Table 2.2. Area, Volume, and Mass Estimates for the 300 Area Uranium Plume

\begin{tabular}{|c|c|c|c|c|c|c|c|c|c|c|c|c|}
\hline \multirow[b]{2}{*}{$\begin{array}{c}\text { Period } \\
\text { Represented }\end{array}$} & \multicolumn{7}{|c|}{ Segment Parameters: } & \multicolumn{5}{|c|}{ Plume Sub-Region Parameters: } \\
\hline & $\begin{array}{l}\text { Segment } \\
\text { (ug/L } \\
\text { contours) }\end{array}$ & $\begin{array}{c}\text { ARCINFO: } \\
\text { Segment } \\
\text { AREA } \\
\left(\mathrm{m}^{2}\right) \\
\end{array}$ & \begin{tabular}{|c|} 
Contam. \\
Thick- \\
ness \\
$(\mathrm{m})$ \\
\end{tabular} & $\begin{array}{l}\text { Segment: } \\
\text { Aquifer } \\
\text { Volume } \\
\text { (m3) }\end{array}$ & $\begin{array}{c}\text { Segment: GW } \\
\text { Volume @ } \\
27 \% \text { porosity } \\
\text { (m3) }\end{array}$ & $\begin{array}{c}\text { Segment: } \\
\text { Average } \\
\text { Uranium } \\
\text { Conc. } \\
\text { (ug/L) }\end{array}$ & $\begin{array}{l}\text { Segment: } \\
\text { Mass of } \\
\text { Uranium } \\
(\mathrm{kg})\end{array}$ & $\begin{array}{c}\text { Plume Sub- } \\
\text { Region }\end{array}$ & $\begin{array}{l}\text { Plume } \\
\text { Sub- } \\
\text { Region } \\
\text { Mass } \\
\text { (kg) }\end{array}$ & $\begin{array}{c}\text { Plume Sub- } \\
\text { Region Area } \\
(\mathrm{m} 2)\end{array}$ & $\begin{array}{c}\text { Sub- } \\
\text { Region } \\
\text { Area } \\
(\mathbf{k m 2}) \\
\end{array}$ & $\begin{array}{c}\text { Sub-Region } \\
\text { GW Volume } \\
\text { (m3) }\end{array}$ \\
\hline September 2001 & $10-20$ & 507,416 & 3.0 & $1,522,248$ & 411,007 & 15 & 6.2 & $>10$ & 35.6 & 943,737 & 0.94 & 764,427 \\
\hline September 2001 & $20-30$ & 73,018 & 3.0 & 219,054 & 59,145 & 25 & 1.5 & $>20$ & 29.4 & 436,321 & 0.44 & 353,420 \\
\hline September 2001 & $30-50$ & 53,711 & 3.0 & 161,132 & 43,506 & 40 & 1.7 & $>30$ & 27.9 & 363,302 & 0.36 & 294,275 \\
\hline September 2001 & $50-100$ & 194,718 & 3.0 & 584,155 & 157,722 & 75 & 11.8 & $>50$ & 26.2 & 309,592 & 0.31 & 250,769 \\
\hline September 2001 & $100-150$ & 47,361 & 3.0 & 142,084 & 38,363 & 125 & 4.8 & $>100$ & 14.4 & 114,873 & 0.11 & 93,047 \\
\hline September 2001 & $150-200$ & 67,512 & 3.0 & 202,536 & 54,685 & 175 & 9.6 & $>150$ & 9.6 & 67,512 & 0.07 & 54,685 \\
\hline June 2002 & $10-20$ & 350,507 & 3.3 & $1,139,148$ & 307,570 & 15 & 4.6 & $>10$ & 27.8 & $1,012,773$ & 1.01 & 888,708 \\
\hline June 2002 & $20-30$ & 246,129 & 3.3 & 799,919 & 215,978 & 25 & 5.4 & $>20$ & 23.2 & 662,265 & 0.66 & 581,138 \\
\hline June 2002 & $30-50$ & 292,726 & 3.3 & 951,361 & 256,867 & 40 & 10.3 & $>30$ & 17.8 & 416,136 & 0.42 & 365,160 \\
\hline June 2002 & $50-80(1)$ & 14,205 & 3.3 & 46,165 & 12,465 & 65 & 0.8 & $>50(1)$ & 7.5 & 123,410 & 0.12 & 108,292 \\
\hline June 2002 & $50-80(2)$ & 86,248 & 3.3 & 280,307 & 75,683 & 65 & 4.9 & $>50(2)$ & 6.7 & 109,205 & 0.11 & 95,828 \\
\hline June 2002 & $80-100$ & 22,957 & 3.3 & 74,610 & 20,145 & 90 & 1.8 & $>80$ & 1.8 & 22,957 & 0.02 & 20,145 \\
\hline December 2002 & $10-20$ & 224,792 & 3.0 & 674,376 & 182,082 & 15 & 2.7 & $>10$ & 37.3 & 862,593 & 0.86 & 698,700 \\
\hline December 2002 & $20-30$ & 204,523 & 3.0 & 613,570 & 165,664 & 25 & 4.1 & $>20$ & 34.5 & 637,801 & 0.64 & 516,618 \\
\hline December 2002 & $30-60$ & 113,094 & 3.0 & 339,281 & 91,606 & 45 & 4.1 & $>30$ & 30.4 & 433,277 & 0.43 & 350,954 \\
\hline December 2002 & $60-90$ & 154,724 & 3.0 & 464,172 & 125,327 & 75 & 9.4 & $>60$ & 26.3 & 320,183 & 0.32 & 259,348 \\
\hline December 2002 & $90-120(1)$ & 11,932 & 3.0 & 35,795 & 9,665 & 105 & 1.0 & $>90(1)$ & 16.9 & 165,459 & 0.17 & 134,022 \\
\hline December 2002 & $90-120(2)$ & 67,571 & 3.0 & 202,712 & 54,732 & 105 & 5.7 & $>90(2)$ & 15.9 & 153,528 & 0.15 & 124,357 \\
\hline December 2002 & $120-150(1)$ & 10,161 & 3.0 & 30,483 & 8,230 & 135 & 1.1 & $>120(1)$ & 10.1 & 85,957 & 0.09 & 69,625 \\
\hline December 2002 & $120-150(2)$ & 59,120 & 3.0 & 177,360 & 47,887 & 135 & 6.5 & $>120(2)$ & 9.0 & 75,796 & 0.08 & 61,395 \\
\hline December 2002 & $150-180$ & 7,456 & 3.0 & 22,368 & 6,039 & 165 & 1.0 & $>150$ & 2.5 & 16,676 & 0.02 & 13,507 \\
\hline December 2002 & $180-210$ & 5,540 & 3.0 & 16,619 & 4,487 & 195 & 0.9 & $>180$ & 1.5 & 9,220 & 0.01 & 7,468 \\
\hline December 2002 & $210-240$ & 3,680 & 3.0 & 11,040 & 2,981 & 225 & 0.7 & $>210$ & 0.7 & 3,680 & 0.00 & 2,981 \\
\hline June 2003 & $10-20$ & 290,691 & 3.3 & 944,746 & 255,081 & 15 & 3.8 & $>10$ & 26.3 & 867,976 & 0.87 & 761,649 \\
\hline June 2003 & $20-30$ & 158,395 & 3.3 & 514,782 & 138,991 & 25 & 3.5 & $>20$ & 22.5 & 577,285 & 0.58 & 506,568 \\
\hline June 2003 & $30-60$ & 336,399 & 3.3 & $1,093,297$ & 295,190 & 45 & 13.3 & $>30$ & 19.0 & 418,891 & 0.42 & 367,577 \\
\hline June 2003 & $60-90(1)$ & 14,679 & 3.3 & 47,708 & 12,881 & 75 & 1.0 & $>60(1)$ & 5.8 & 82,492 & 0.08 & 72,387 \\
\hline June 2003 & $60-90(2)$ & 27,363 & 3.3 & 88,929 & 24,011 & 75 & 1.8 & $>60(2)$ & 4.8 & 67,812 & 0.07 & 59,505 \\
\hline June 2003 & $60-90(3)$ & 27,874 & 3.3 & 90,592 & 24,460 & 75 & 1.8 & $>60(3)$ & 3.0 & 40,450 & 0.04 & 35,494 \\
\hline June 2003 & $90-120$ & 12,575 & 3.3 & 40,869 & 11,035 & 105 & 1.2 & $>90$ & 1.2 & 12,575 & 0.01 & 11,035 \\
\hline December 2003 & $10-20$ & 394,383 & 3.0 & $1,183,148$ & 319,450 & 15 & 4.8 & $>10$ & 23.6 & 869,575 & 0.87 & 704,355 \\
\hline December 2003 & $20-30$ & 151,470 & 3.0 & 454,409 & 122,691 & 25 & 3.1 & $>20$ & 18.8 & 475,192 & 0.48 & 384,905 \\
\hline December 2003 & $30-60$ (1) & 181,659 & 3.0 & 544,978 & 147,144 & 45 & 6.6 & $>30(1)$ & 15.7 & 323,722 & 0.32 & 262,215 \\
\hline December 2003 & $30-60(2)$ & 5,113 & 3.0 & 15,340 & 4,142 & 45 & 0.2 & $>30(2)$ & 9.1 & 142,063 & 0.14 & 115,071 \\
\hline December 2003 & $60-90$ & 112,759 & 3.0 & 338,278 & 91,335 & 75 & 6.9 & $>60$ & 8.9 & 136,950 & 0.14 & 110,929 \\
\hline December 2003 & $90-120$ & 24,190 & 3.0 & 72,571 & 19,594 & 105 & 2.1 & $>90$ & 2.1 & 24,190 & 0.02 & 19,594 \\
\hline
\end{tabular}


Table 2.2. (contd)

\begin{tabular}{|c|c|c|c|c|c|c|c|c|c|c|c|c|}
\hline \multirow[b]{2}{*}{$\begin{array}{c}\text { Period } \\
\text { Represented }\end{array}$} & \multicolumn{7}{|c|}{ Segment Parameters: } & \multicolumn{5}{|c|}{ Plume Sub-Region Parameters: } \\
\hline & $\begin{array}{l}\text { Segment } \\
\text { (ug/L } \\
\text { contours) }\end{array}$ & $\begin{array}{l}\text { ARCINFO: } \\
\text { Segment } \\
\text { AREA } \\
\left(\mathrm{m}^{2}\right) \\
\end{array}$ & $\begin{array}{c}\text { Contam. } \\
\text { Thick- } \\
\text { ness } \\
(m)\end{array}$ & $\begin{array}{l}\text { Segment: } \\
\text { Aquifer } \\
\text { Volume } \\
\text { (m3) }\end{array}$ & $\begin{array}{c}\text { Segment: GW } \\
\text { Volume @ } \\
27 \% \text { porosity } \\
\text { (m3) }\end{array}$ & $\begin{array}{c}\text { Segment: } \\
\text { Average } \\
\text { Uranium } \\
\text { Conc. } \\
\text { (ug/L) }\end{array}$ & $\begin{array}{l}\text { Segment: } \\
\text { Mass of } \\
\text { Uranium } \\
(\mathrm{kg})\end{array}$ & $\begin{array}{c}\text { Plume Sub- } \\
\text { Region }\end{array}$ & $\begin{array}{l}\text { Plume } \\
\text { Sub- } \\
\text { Region } \\
\text { Mass } \\
\text { (kg) }\end{array}$ & $\begin{array}{c}\text { Plume Sub- } \\
\text { Region Area } \\
(\mathrm{m} 2)\end{array}$ & $\begin{array}{c}\text { Sub- } \\
\text { Region } \\
\text { Area } \\
(\mathbf{k m 2}) \\
\end{array}$ & $\begin{array}{c}\text { Sub-Region } \\
\text { GW Volume } \\
\text { (m3) }\end{array}$ \\
\hline September 2001 & $10-20$ & 507,416 & 3.0 & $1,522,248$ & 411,007 & 15 & 6.2 & $>10$ & 35.6 & 943,737 & 0.94 & 764,427 \\
\hline September 2001 & $20-30$ & 73,018 & 3.0 & 219,054 & 59,145 & 25 & 1.5 & $>20$ & 29.4 & 436,321 & 0.44 & 353,420 \\
\hline September 2001 & $30-50$ & 53,711 & 3.0 & 161,132 & 43,506 & 40 & 1.7 & $>30$ & 27.9 & 363,302 & 0.36 & 294,275 \\
\hline September 2001 & $50-100$ & 194,718 & 3.0 & 584,155 & 157,722 & 75 & 11.8 & $>50$ & 26.2 & 309,592 & 0.31 & 250,769 \\
\hline September 2001 & $100-150$ & 47,361 & 3.0 & 142,084 & 38,363 & 125 & 4.8 & $>100$ & 14.4 & 114,873 & 0.11 & 93,047 \\
\hline September 2001 & $150-200$ & 67,512 & 3.0 & 202,536 & 54,685 & 175 & 9.6 & $>150$ & 9.6 & 67,512 & 0.07 & 54,685 \\
\hline June 2002 & $10-20$ & 350,507 & 3.3 & $1,139,148$ & 307,570 & 15 & 4.6 & $>10$ & 27.8 & $1,012,773$ & 1.01 & 888,708 \\
\hline June 2002 & $20-30$ & 246,129 & 3.3 & 799,919 & 215,978 & 25 & 5.4 & $>20$ & 23.2 & 662,265 & 0.66 & 581,138 \\
\hline June 2002 & $30-50$ & 292,726 & 3.3 & 951,361 & 256,867 & 40 & 10.3 & $>30$ & 17.8 & 416,136 & 0.42 & 365,160 \\
\hline June 2004 & $10-30$ & 456,106 & 3.3 & $1,482,345$ & 400,233 & 20 & 8.0 & $>10$ & 30.1 & 851,862 & 0.85 & 747,509 \\
\hline June 2004 & $30-60$ & 200,102 & 3.3 & 650,332 & 175,590 & 45 & 7.9 & $>30$ & 22.1 & 395,756 & 0.40 & 347,276 \\
\hline June 2004 & $60-90$ & 136,567 & 3.3 & 443,844 & 119,838 & 75 & 9.0 & $>60$ & 14.2 & 195,654 & 0.20 & 171,686 \\
\hline June 2004 & $90-110(1)$ & 52,070 & 3.3 & 169,227 & 45,691 & 100 & 4.6 & $>90(1)$ & 5.2 & 59,087 & 0.06 & 51,848 \\
\hline June 2004 & $90-110(2)$ & 7,017 & 3.3 & 22,804 & 6,157 & 100 & 0.6 & $>90(2)$ & 0.6 & 7,017 & 0.01 & 6,157 \\
\hline December 2004 & $10-30$ & 549,485 & 3.0 & $1,648,455$ & 445,083 & 20 & 8.9 & $>10$ & 30.5 & 951,658 & 0.95 & 770,843 \\
\hline December 2004 & $30-60$ & 165,737 & 3.0 & 497,212 & 134,247 & 45 & 6.0 & $>30$ & 21.6 & 402,173 & 0.40 & 325,760 \\
\hline December 2004 & $60-90(1)$ & 171,852 & 3.0 & 515,557 & 139,200 & 75 & 10.4 & $>60(1)$ & 15.6 & 236,436 & 0.24 & 191,513 \\
\hline December 2004 & $60-90(2)$ & 5,288 & 3.0 & 15,863 & 4,283 & 75 & 0.3 & $>60(2)$ & 5.1 & 64,584 & 0.06 & 52,313 \\
\hline December 2004 & $90-110$ & 59,296 & 3.0 & 177,888 & 48,030 & 100 & 4.8 & $>90$ & 4.8 & 59,296 & 0.06 & 48,030 \\
\hline
\end{tabular}


Table 2.3. Summary of Uranium Plume Parameters

\begin{tabular}{|c|c|c|c|c|c|c|}
\hline \multirow[b]{2}{*}{$\begin{array}{c}\text { Period } \\
\text { Represented }\end{array}$} & \multicolumn{3}{|c|}{ >30 $\mu \mathrm{g} / \mathrm{L}$ Portion of Plume: } & \multicolumn{3}{|c|}{$>10 \mu \mathrm{g} / \mathrm{L}$ Portion of Plume: } \\
\hline & $\begin{array}{l}\text { Area of } \\
\text { Plume } \\
\left(\mathrm{km}^{2}\right)\end{array}$ & $\begin{array}{c}\text { Volume of } \\
\text { Water } \\
\left(\mathrm{m}^{3}\right)\end{array}$ & $\begin{array}{l}\text { Mass of } \\
\text { Uranium } \\
(\mathrm{kg})\end{array}$ & $\begin{array}{l}\text { Area of } \\
\text { Plume } \\
\left(\mathrm{km}^{2}\right)\end{array}$ & $\begin{array}{c}\text { Volume of } \\
\text { Water } \\
\left(\mathrm{m}^{3}\right)\end{array}$ & $\begin{array}{c}\text { Mass of } \\
\text { Uranium } \\
\text { (kg) }\end{array}$ \\
\hline September 2001 & 0.36 & 294,275 & 27.9 & 0.94 & 764,427 & 35.6 \\
\hline June 2002 & 0.42 & 365,160 & 17.8 & 1.01 & 888,708 & 27.8 \\
\hline December 2002 & 0.43 & 350,954 & 30.4 & 0.86 & 698,700 & 37.3 \\
\hline June 2003 & 0.42 & 367,577 & 19.0 & 0.87 & 761,649 & 26.3 \\
\hline December 2003 & 0.46 & 377,286 & 24.8 & 0.87 & 704,355 & 23.6 \\
\hline June 2004 & 0.40 & 347,276 & 19.2 & 0.85 & 747,509 & 29.2 \\
\hline December 2004 & 0.40 & 325,760 & 21.6 & 0.95 & 770,843 & 30.5 \\
\hline \multicolumn{7}{|c|}{$\begin{array}{l}\text { Assumptions: } \\
\text { 1. Contaminated thickness: } 3.0 \text { meters (December) and } 3.3 \text { meters (June). } \\
\text { 2. Effective porosity estimated at } 27 \% \text {. } \\
\text { 3. Mass is estimated using mid-point concentration between contours. }\end{array}$} \\
\hline
\end{tabular}

\subsubsection{Cis-1,2-Dichloroethene}

The maximum concentrations of cis-1,2-dichloroethene are generally well below the drinking water standard (70 $\mu \mathrm{g} / \mathrm{L})$ or non-detected at all 300 Area wells and shoreline monitoring sites (Table 2.4). The exception occurs at well 399-1-16B, which monitors the bottom of the unconfined aquifer between the 300 Area process trenches, the presumed source, and the Columbia River. Detections of cis-1,2dichloroethene occur at several other wells that monitor the bottom of the unconfined aquifer near the 300 Area process trenches, and also at the aquifer tube site located along the downgradient flow path from well 399-1-16B (tube site AT-3-3). These detections are at very low concentrations, i.e., at less than $3 \mu \mathrm{g} / \mathrm{L}$. The concentration trend at well 399-1-16B has remained essentially constant since 1992, and in the range 120 to $190 \mu \mathrm{g} / \mathrm{L}$.

Groundwater flow at the bottom of the unconfined aquifer is probably much slower than at the top, which may contribute to the persistence of this constituent at this location. The origin for cis-1,2dichloroethene is not precisely known; it may have been included in liquid effluent discharged to the 300 Area process trenches, or it may be a degradation product of another organic compound in the discharge, e.g., tetrachloroethene (Lindberg and Chou 2001). Values for cis-1,2-dichloroethene (total), which were obtained during the initial limited field investigation for 300-FF-5, are shown in Table 2.5.

\subsubsection{Trichloroethene}

The maximum concentrations of trichloroethene are generally below the drinking water standard (5 $\mu \mathrm{g} / \mathrm{L})$ at all 300 Area wells and at aquifer tube sites along the shoreline (Table 2.6), with two exceptions for recent sampling events:

- Values above the standard were observed at well 399-1-7, located between the 300 Area process trenches and Columbia River, during 2004 (5.4 $\mu \mathrm{g} / \mathrm{L})$ and $2003(7.2 \mu \mathrm{g} / \mathrm{L})$. 
- A value of $6.8 \mu \mathrm{g} / \mathrm{L}$ was also observed during 2004 at aquifer tube site AT-3-3, which is located along the downgradient groundwater flow path from the process trenches and well 399-1-7.

Trichloroethene was introduced to 300 Area groundwater via discharge to the 300 Area process trenches, and it also migrates into the 300 Area from sources to the southwest of the 300 Area (Lindberg and Peterson 2004). Where trichloroethene was present in groundwater above the drinking water standard in the past, concentrations have generally decreased with time. For example, see trends revealed in Table 2.6 for wells 399-2-2, 399-4-12, and 399-1-16B. (Note: A result of $28 \mu \mathrm{g} / \mathrm{L}$ at an unconfined aquifer well (699-S29-E12) to the southwest of the 300 Area, and of $22 \mu \mathrm{g} / \mathrm{L}$ at confined aquifer well 399-1-16C, are believed to be outliers and not representative of aquifer conditions.)

\subsubsection{Other Constituents of Potential Concern}

Tetrachloroethene - Maximum values for tetrachloroethene at all 300 Area wells and shoreline aquifer tube sites are well below the $5-\mu \mathrm{g} / \mathrm{L}$ drinking water standard (i.e., they are less than $1 \mu \mathrm{g} / \mathrm{L}$ or non-detected) during recent years (Table 2.7). Tetrachloroethene degrades to cis-1,2-dichloroethene and trichloroethene in a reducing environment.

Strontium-90 - This radionuclide, with a half-life of 28 years, has been detected at a concentration above the 8-pCi/L drinking water standard once during monitoring since 1992, and only at well 399-3-11, which is located near the 307 trench (Table 2.8; see also Figure 2.6 for well locations). Strontium-90 concentrations at well 399-3-11 have remained essentially constant since the exceedance noted in 1995, with the most recent values between 3 and $4 \mathrm{pCi} / \mathrm{L}$. Where detected at other wells during the remedial investigation in the 1990s, the concentrations were less than $5 \mathrm{pCi} / \mathrm{L}$.

Tritium - Tritium migrates into the 300 Area from sources to the northwest, as part of the site-wide plume associated with waste sites in the 200 East Area (200-PO-1 Operable Unit). All maximum values since 1992 for all 300 Area wells and aquifer tube sites are below the 20,000-pCi/L drinking water standard (Table 2.9), with the exception of one possible outlier value at well 399-3-12 in 1996.

Nitrate - Maximum concentrations for nitrate exceed the 45-mg/L drinking water standard in several wells located at the southwest perimeter of the 300 Area (Table 2.10). The standard is also exceeded at two aquifer tube sites located at the downstream (south) end of the 300 Area shoreline (see Figure 2.6 for well locations). While some nitrate may have entered the aquifer from 300 Area sources in the past (e.g., septic systems), the current distribution is believed to be dominated by contaminated groundwater that migrates into the 300 Area from industrial and agricultural sources located to the southwest. 
Table 2.4. Maximum Cis-1,2-Dichloroethene Concentrations ( $\mu \mathrm{g} / \mathrm{L})$ at 300 Area Wells

\begin{tabular}{|c|c|c|c|c|c|c|c|c|c|c|c|c|c|c|}
\hline Well Name & Unit & 1992 & 1993 & 1994 & 1995 & 1996 & 1997 & 1998 & 1999 & 2000 & 2001 & 2002 & 2003 & 2004 \\
\hline \multicolumn{15}{|c|}{ Wells that monitor the top of the unconfined aquifer } \\
\hline $399-1-1$ & TU & & & & & & & & & & & ND & ND & ND \\
\hline $399-1-2$ & TU & & $\mathrm{ND}$ & $\mathrm{ND}$ & ND & & & & & & & ND & ND & ND \\
\hline $399-1-3$ & $\mathrm{TU}$ & & & & ND & & & ND & ND & ND & ND & & & \\
\hline $399-1-4$ & TU & & ND & & ND & & & & & & & & & \\
\hline 399-1-6 & $\mathrm{TU}$ & & ND & $\mathrm{ND}$ & ND & & & & & & & & ND & ND \\
\hline 399-1-7 & $\mathrm{TU}$ & & & & & & & & & & ND & 0.6 & 0.7 & 0.5 \\
\hline 399-1-10A & $\mathrm{TU}$ & & & $\mathrm{ND}$ & ND & ND & 0.0 & ND & ND & 0.4 & ND & ND & 0.2 & ND \\
\hline 399-1-11 & TU & & & ND & ND & ND & & & & & ND & ND & ND & ND \\
\hline 399-1-12 & $\mathrm{TU}$ & & & $\mathrm{ND}$ & ND & 0.2 & & & & & & 0.3 & 0.2 & ND \\
\hline 399-1-14A & $\mathrm{TU}$ & & & $\mathrm{ND}$ & ND & ND & ND & ND & ND & ND & ND & & & \\
\hline 399-1-15 & $\mathrm{TU}$ & & & & & & & & & & & ND & ND & ND \\
\hline 399-1-16A & $\mathrm{TU}$ & & & $\mathrm{ND}$ & ND & 0.2 & 0.3 & 0.7 & 0.3 & 0.6 & 0.2 & 0.5 & 0.4 & 0.4 \\
\hline 399-1-17A & $\mathrm{TU}$ & & & $\mathrm{ND}$ & $\mathrm{ND}$ & ND & 0.1 & 5.0 & 0.2 & ND & ND & ND & 0.2 & 0.3 \\
\hline 399-1-18A & $\mathrm{TU}$ & & & $\mathrm{ND}$ & ND & ND & ND & ND & ND & ND & ND & & ND & ND \\
\hline 399-1-19 & $\mathrm{TU}$ & & & ND & 0.3 & & (a) & (a) & (a) & (a) & (a) & (a) & (a) & (a) \\
\hline 399-1-21A & $\mathrm{TU}$ & & ND & $\mathrm{ND}$ & ND & & ND & ND & ND & ND & ND & ND & ND & ND \\
\hline 399-2-1 & $\mathrm{TU}$ & & & $\mathrm{ND}$ & ND & ND & ND & ND & 0.4 & & ND & 0.4 & 0.1 & 0.1 \\
\hline $399-2-2$ & $\mathrm{TU}$ & & 0.3 & 0.8 & 0.2 & ND & 0.4 & 0.8 & 0.7 & ND & 0.6 & 0.7 & 0.6 & 0.6 \\
\hline 399-2-3 & $\mathrm{TU}$ & & 0.2 & 0.6 & ND & ND & & & & & & & & \\
\hline 399-3-1 & $\mathrm{TU}$ & & & 0.3 & ND & ND & & & & & & & & \\
\hline 399-3-2 & $\mathrm{TU}$ & & & $\mathrm{ND}$ & ND & & ND & ND & ND & ND & & ND & ND & 0.2 \\
\hline 399-3-3 & $\mathrm{TU}$ & & & $\mathrm{ND}$ & & ND & $\mathrm{ND}$ & & $\mathrm{ND}$ & ND & & & & \\
\hline 399-3-6 & $\mathrm{TU}$ & & & ND & ND & ND & ND & ND & ND & ND & & 0.5 & ND & ND \\
\hline 399-3-9 & $\mathrm{TU}$ & & & ND & & & & & & & & & & \\
\hline 399-3-10 & $\mathrm{TU}$ & & & $\mathrm{ND}$ & ND & $\mathrm{ND}$ & $\mathrm{ND}$ & ND & $\mathrm{ND}$ & ND & ND & ND & ND & ND \\
\hline 399-3-11 & $\mathrm{TU}$ & & & $\mathrm{ND}$ & ND & ND & ND & ND & ND & ND & ND & ND & ND & ND \\
\hline 399-3-12 & $\mathrm{TU}$ & & ND & $\mathrm{ND}$ & & & & & & & & ND & ND & ND \\
\hline 399-4-1 & $\mathrm{TU}$ & & & $\mathrm{ND}$ & ND & ND & ND & ND & ND & ND & ND & ND & ND & ND \\
\hline 399-4-7 & $\mathrm{TU}$ & & & & ND & ND & & & & ND & ND & & & \\
\hline 399-4-9 & $\mathrm{TU}$ & & ND & $\mathrm{ND}$ & & & & & ND & ND & ND & ND & 0.1 & ND \\
\hline $399-4-10$ & $\mathrm{TU}$ & & ND & $\mathrm{ND}$ & ND & & & & ND & ND & ND & ND & & ND \\
\hline 399-4-11 & $\mathrm{TU}$ & & & $\mathrm{ND}$ & ND & ND & & & 0.4 & ND & ND & ND & $\mathrm{ND}$ & \\
\hline $399-4-12$ & $\mathrm{TU}$ & & & & ND & & ND & ND & ND & ND & ND & ND & ND & ND \\
\hline 399-5-1 & $\mathrm{TU}$ & & ND & ND & ND & ND & ND & ND & ND & ND & ND & & & \\
\hline 399-5-4B & $\mathrm{TU}$ & & & & ND & ND & ND & ND & ND & ND & ND & ND & ND & ND \\
\hline 399-6-1 & $\mathrm{TU}$ & & & & ND & ND & ND & ND & ND & ND & & & & \\
\hline 399-6-2 & $\mathrm{TU}$ & & & & ND & ND & $\mathrm{ND}$ & ND & $\mathrm{ND}$ & ND & ND & ND & $\mathrm{ND}$ & \\
\hline 399-8-1 & $\mathrm{TU}$ & & & $\mathrm{ND}$ & ND & ND & ND & ND & ND & ND & ND & & & \\
\hline 399-8-5A & $\mathrm{TU}$ & & ND & $\mathrm{ND}$ & & ND & ND & ND & ND & ND & ND & ND & ND & ND \\
\hline 699-S19-E13 & $\mathrm{TU}$ & ND & & & $\mathrm{ND}$ & & & & & & & & & \\
\hline 699-S19-E14 & $\mathrm{TU}$ & & $\mathrm{ND}$ & & & & & & & & & & & \\
\hline 699-S27-E12A & $\mathrm{TU}$ & & & & & & & ND & ND & ND & ND & ND & ND & ND \\
\hline 699-S27-E14 & $\mathrm{TU}$ & ND & ND & $\mathrm{ND}$ & ND & ND & ND & ND & ND & ND & ND & ND & ND & ND \\
\hline 699-S28-E13A & $\mathrm{TU}$ & & & & & & & ND & ND & ND & ND & ND & ND & ND \\
\hline 699-S29-E12 & $\mathrm{TU}$ & ND & ND & $\mathrm{ND}$ & ND & ND & $\mathrm{ND}$ & ND & ND & & & ND & ND & ND \\
\hline 699-S29-E13A & $\mathrm{TU}$ & & & & & & & ND & $\mathrm{ND}$ & ND & ND & ND & ND & ND \\
\hline 699-S29-E16A & $\mathrm{TU}$ & & $\mathrm{ND}$ & $\mathrm{ND}$ & ND & ND & ND & ND & & ND & ND & ND & ND & ND \\
\hline 699-S30-E15A & $\mathrm{TU}$ & ND & & & & & & & & & & & & \\
\hline
\end{tabular}


Table 2.4. (contd)

\begin{tabular}{|c|c|c|c|c|c|c|c|c|c|c|c|c|c|c|}
\hline Well Name & Unit & 1992 & 1993 & 1994 & 1995 & 1996 & 1997 & 1998 & 1999 & 2000 & 2001 & 2002 & 2003 & 2004 \\
\hline \multicolumn{15}{|c|}{ Aquifer tubes at the shoreline that monitor the top of the unconfined aquifer } \\
\hline AT-3-1-D(1) & TU-S & & & & & & & & & & & & & ND \\
\hline AT-3-2-M & TU-S & & & & & & & & & & & & & ND \\
\hline AT-3-3-M & TU-S & & & & & & & & & & & & & 0.2 \\
\hline AT-3-4-S & TU-S & & & & & & & & & & & & & ND \\
\hline AT-3-5-S & TU-S & & & & & & & & & & & & & ND \\
\hline AT-3-6-S & TU-S & & & & & & & & & & & & & ND \\
\hline AT-3-7-M & TU-S & & & & & & & & & & & & & ND \\
\hline AT-3-8-S & TU-S & & & & & & & & & & & & & ND \\
\hline \multicolumn{15}{|c|}{ Wells that monitor the bottom of the unconfined aquifer } \\
\hline $399-1-8$ & BU & & 0.3 & 0.2 & & & & & & & ND & ND & 0.4 & 0.1 \\
\hline 399-1-10B & BU & ND & ND & ND & ND & ND & ND & ND & ND & 0.3 & ND & ND & ND & 0.2 \\
\hline 399-1-13B & BU & ND & ND & ND & & & & & & & & & & \\
\hline 399-1-14B & $\mathrm{BU}$ & ND & ND & ND & ND & & ND & & & & & & & \\
\hline $399-1-16 B$ & BU & 120.0 & & 130.0 & 147.0 & 170.0 & 190.0 & 180.0 & 180.0 & 170.0 & 190.0 & 160.0 & 160.0 & 150.0 \\
\hline 399-1-17B & $\mathrm{BU}$ & & & 1.5 & 2.8 & 0.8 & 3.0 & 3.0 & 4.4 & 4.7 & 3.3 & 1.4 & 2.8 & 3.3 \\
\hline 399-1-18B & $\mathrm{BU}$ & & & & & ND & ND & ND & ND & ND & ND & & ND & ND \\
\hline $399-1-21 B$ & $\mathrm{BU}$ & & ND & ND & ND & & & & & & ND & ND & ND & ND \\
\hline 699-S29-E16B & $\mathrm{BU}$ & & ND & ND & ND & & & & & & & & & \\
\hline \multicolumn{15}{|c|}{ Wells that monitor the uppermost confined aquifer } \\
\hline $399-1-9$ & C & & ND & ND & & & & & & & & & & \\
\hline $399-1-16 C$ & $\mathrm{C}$ & 60.0 & & & ND & ND & & & & & & & & \\
\hline $399-8-5 B$ & $\mathrm{C}$ & & ND & ND & ND & & & & & & & & & \\
\hline $399-8-5 C$ & $\mathrm{C}$ & & ND & ND & ND & & & & & & & & & \\
\hline 699-S29-E16C & $\mathrm{C}$ & & ND & ND & ND & & & & & & & & & \\
\hline \multicolumn{15}{|c|}{$\begin{array}{l}\text { (a) Well out-of-service. } \\
\text { Note: Maximum values for cis-1,2-dichloroethene at } 300 \text { Area wells. Values in bold in shaded cells are below the EPA drinking water } \\
\text { standard }(70 \mu \mathrm{g} / \mathrm{L}) \text {. Blanks indicate "no results." } \\
\text { BU }=\text { Bottom of unconfined aquifer. } \\
\mathrm{C}=\text { First confined aquifer. } \\
\text { EPA }=\text { U.S. Environmental Protection Agency. } \\
\text { ND }=\text { Not detected. } \\
\text { TU }=\text { Top of unconfined aquifer. } \\
\text { TU-S= Top of unconfined aquifer at shoreline. }\end{array}$} \\
\hline
\end{tabular}


Table 2.5. Maximum Total 1,2-Dichloroethene Concentrations ( $\mu \mathrm{g} / \mathrm{L})$ at 300 Area Wells

\begin{tabular}{|c|c|c|c|c|c|c|c|c|c|c|c|c|c|c|}
\hline Well Name & Unit & 1992 & 1993 & 1994 & 1995 & 1996 & 1997 & 1998 & 1999 & 2000 & 2001 & 2002 & 2003 & 2004 \\
\hline \multicolumn{15}{|c|}{ Wells that monitor the top of the unconfined aquifer } \\
\hline $399-1-5$ & TU & ND & ND & ND & & & & & & (a) & (a) & (a) & (a) & (a) \\
\hline $399-1-6$ & $\mathrm{TU}$ & ND & & & & & & & & & & & & \\
\hline $399-1-7$ & TU & ND & ND & ND & & & & & & & & & & \\
\hline $399-1-10 \mathrm{~A}$ & $\mathrm{TU}$ & ND & ND & & & & & & & & & & & \\
\hline $399-1-11$ & $\mathrm{TU}$ & ND & ND & & & & & & & & & & & \\
\hline 399-1-12 & $\mathrm{TU}$ & ND & ND & & & & & & & & & & & \\
\hline 399-1-13A & $\mathrm{TU}$ & ND & ND & & & & & & & & & & & \\
\hline $399-1-14 \mathrm{~A}$ & $\mathrm{TU}$ & ND & ND & & & & & & & & & & & \\
\hline 399-1-15 & $\mathrm{TU}$ & ND & $\mathrm{ND}$ & & & & & & & & & & & \\
\hline 399-1-16A & $\mathrm{TU}$ & ND & 31.0 & & & & & & & & & & & \\
\hline 399-1-17A & $\mathrm{TU}$ & ND & ND & & & & & & & & & & & \\
\hline 399-1-18A & $\mathrm{TU}$ & ND & ND & & & & & & & & & & & \\
\hline $399-1-21 \mathrm{~A}$ & $\mathrm{TU}$ & ND & ND & ND & & & & & & & & & & \\
\hline $399-2-1$ & $\mathrm{TU}$ & ND & ND & & & & & & & & & & & \\
\hline $399-2-2$ & $\mathrm{TU}$ & ND & $\mathrm{ND}$ & $\mathrm{ND}$ & & & & & & & & & & \\
\hline $399-2-3$ & $\mathrm{TU}$ & ND & & & & & & & & & & & & \\
\hline 399-3-2 & $\mathrm{TU}$ & ND & ND & ND & & & & & & & & & & \\
\hline 399-3-3 & $\mathrm{TU}$ & ND & & & & & & & & & & & & \\
\hline 399-3-9 & $\mathrm{TU}$ & ND & & & & & & & & & & & & \\
\hline 399-3-10 & $\mathrm{TU}$ & ND & ND & & & & & & & & & & & \\
\hline 399-3-11 & $\mathrm{TU}$ & ND & ND & ND & & & & & & & & & & \\
\hline 399-3-12 & $\mathrm{TU}$ & ND & $\mathrm{ND}$ & ND & & & & & & & & & & \\
\hline $399-4-1$ & $\mathrm{TU}$ & ND & ND & & & & & & & & & & & \\
\hline $399-4-7$ & $\mathrm{TU}$ & ND & ND & ND & & & & & & & & & & \\
\hline 399-4-9 & $\mathrm{TU}$ & ND & & & & & & & & & & & & \\
\hline 399-4-10 & $\mathrm{TU}$ & ND & ND & $\mathrm{ND}$ & & & & & & & & & & \\
\hline 399-4-11 & $\mathrm{TU}$ & ND & ND & & & & & & & & & & & \\
\hline 399-4-12 & $\mathrm{TU}$ & ND & $\mathrm{ND}$ & ND & & & & & & & & & & \\
\hline 399-5-1 & $\mathrm{TU}$ & ND & ND & & & & & & & & & & & \\
\hline 399-6-1 & $\mathrm{TU}$ & ND & ND & & & & & & & & & & & \\
\hline 399-8-1 & $\mathrm{TU}$ & ND & ND & & & & & & & & & & & \\
\hline $399-8-2$ & $\mathrm{TU}$ & ND & & & & & & & & & & & & \\
\hline 399-8-3 & $\mathrm{TU}$ & ND & ND & & & & & & & & & & & \\
\hline 399-8-4 & $\mathrm{TU}$ & ND & & & & & & & & & & & & \\
\hline $399-8-5 A$ & $\mathrm{TU}$ & ND & ND & & & & & & & & & & & \\
\hline 699-S19-E14 & $\mathrm{TU}$ & ND & & & & & & & & & & & & \\
\hline 699-S27-E12A & $\mathrm{TU}$ & & & & & ND & ND & ND & & & & & & \\
\hline 699-S27-E14 & $\mathrm{TU}$ & ND & ND & & & & & & & & & & & \\
\hline 699-S28-E13A & $\mathrm{TU}$ & & & & & ND & ND & $\mathrm{ND}$ & & & & & & \\
\hline 699-S29-E12 & $\mathrm{TU}$ & & ND & & & ND & & & & & & & & \\
\hline 699-S29-E13A & $\mathrm{TU}$ & & & & & ND & ND & ND & & & & & & \\
\hline 699-S29-E16A & $\mathrm{TU}$ & ND & & & & & & & & & & & & \\
\hline 699-S30-E15A & $\mathrm{TU}$ & ND & & & & & & & & & & & & \\
\hline
\end{tabular}


Table 2.5. (contd)

\begin{tabular}{|c|c|c|c|c|c|c|c|c|c|c|c|c|c|c|}
\hline Well Name & Unit & 1992 & 1993 & 1994 & 1995 & 1996 & 1997 & 1998 & 1999 & 2000 & 2001 & 2002 & 2003 & 2004 \\
\hline \multicolumn{15}{|c|}{ Wells that monitor the bottom of the unconfined aquifer } \\
\hline $399-1-8$ & BU & ND & & & & & & & & & & & & \\
\hline 399-1-10B & $\mathrm{BU}$ & ND & & & & & & & & & & & & \\
\hline 399-1-13B & BU & ND & & & & & & & & & & & & \\
\hline $399-1-14 B$ & BU & ND & & & & & & & & & & & & \\
\hline $399-1-16 B$ & $\mathrm{BU}$ & 130.0 & 180.0 & & & & & & & & & & & \\
\hline 399-1-17B & BU & 6.0 & 5.1 & & & & & & & & & & & \\
\hline 399-1-18B & $\mathrm{BU}$ & ND & & & & & & & & & & & & \\
\hline 399-1-21B & $\mathrm{BU}$ & ND & & & & & & & & & & & & \\
\hline 699-S29-E16B & BU & ND & & & & & & & & & & & & \\
\hline \multicolumn{15}{|c|}{ Wells that monitor the uppermost confined aquifer } \\
\hline $399-1-9$ & $\mathrm{C}$ & ND & & & & & & & & & & & & \\
\hline $399-1-16 C$ & $\mathrm{C}$ & ND & 4.0 & ND & & & & & & & & & & \\
\hline 399-1-17C & $\mathrm{C}$ & ND & & & & & & & & & & & & \\
\hline 399-1-18C & C & ND & & & & & & & & & & & & \\
\hline 399-8-5B & $\mathrm{C}$ & ND & & & & & & & & & & & & \\
\hline $399-8-5 C$ & $\mathrm{C}$ & ND & & & & & & & & & & & & \\
\hline 699-S29-E16C & $\mathrm{C}$ & ND & & & & & & & & & & & & \\
\hline \multicolumn{15}{|c|}{$\begin{array}{l}\text { (a) Well out-of-service. } \\
\text { Note: Maximum values for 1,2-dichloroethene (total) at } 300 \text { Area wells. Values in bold in shaded cells are below the EPA drinking water } \\
\text { standard }(100 \mu \mathrm{g} / \mathrm{L}) \text {. Blanks indicate “no results.” } \\
\text { BU }=\text { Bottom of unconfined aquifer. } \\
\mathrm{C} \quad=\text { First confined aquifer. } \\
\text { EPA }=\text { U.S. Environmental Protection Agency. } \\
\text { ND }=\text { Not detected. } \\
\text { TU }=\text { Top of unconfined aquifer. } \\
\text { TU-S }=\text { Top of unconfined aquifer at shoreline. }\end{array}$} \\
\hline
\end{tabular}


Table 2.6. Maximum Trichloroethene Concentrations ( $\mu \mathrm{g} / \mathrm{L})$ at 300 Area Wells

\begin{tabular}{|c|c|c|c|c|c|c|c|c|c|c|c|c|c|c|}
\hline Well Name & Unit & 1992 & 1993 & 1994 & 1995 & 1996 & 1997 & 1998 & 1999 & 2000 & 2001 & 2002 & 2003 & 2004 \\
\hline \multicolumn{15}{|c|}{ Wells that monitor the top of the unconfined aquifer } \\
\hline $399-1-1$ & $\mathrm{TU}$ & & & & & & & & & & & ND & 0.3 & ND \\
\hline $399-1-2$ & TU & & 0.6 & 0.7 & ND & & & & & & & 2.8 & 0.2 & 0.2 \\
\hline $399-1-3$ & TU & & & & 0.6 & & & 2.0 & 0.5 & 0.6 & 0.9 & & & \\
\hline $399-1-4$ & TU & & 0.2 & & ND & & & & & & & & & \\
\hline $399-1-5$ & $\mathrm{TU}$ & $\mathrm{ND}$ & $\mathrm{ND}$ & ND & & & & & & (a) & (a) & (a) & (a) & (a) \\
\hline 399-1-6 & $\mathrm{TU}$ & $\mathrm{ND}$ & $\mathrm{ND}$ & ND & ND & & & & & & & & ND & ND \\
\hline 399-1-7 & $\mathrm{TU}$ & 1.0 & 3.9 & 4.0 & & & & & & & 1.3 & 2.3 & 1.1 & 5.4 \\
\hline 399-1-10A & $\mathrm{TU}$ & $\mathrm{ND}$ & ND & ND & ND & ND & 0.1 & 0.3 & ND & ND & ND & ND & ND & ND \\
\hline 399-1-11 & $\mathrm{TU}$ & $\mathrm{ND}$ & $\mathrm{ND}$ & ND & ND & 0.4 & & & & & ND & ND & ND & ND \\
\hline 399-1-12 & $\mathrm{TU}$ & $\mathrm{ND}$ & 1.2 & 0.3 & 0.2 & 0.5 & & & & & & 0.4 & 0.5 & 0.3 \\
\hline 399-1-13A & $\mathrm{TU}$ & ND & ND & & & & & & & & & & & \\
\hline 399-1-14A & $\mathrm{TU}$ & $\mathrm{ND}$ & $\mathrm{ND}$ & ND & ND & $\mathrm{ND}$ & ND & ND & ND & ND & ND & & & \\
\hline 399-1-15 & $\mathrm{TU}$ & $\mathrm{ND}$ & $\mathrm{ND}$ & & & & & & & & & ND & ND & $\mathrm{ND}$ \\
\hline 399-1-16A & $\mathrm{TU}$ & 3.0 & 2.7 & 1.1 & 0.9 & 0.9 & 1.0 & 1.0 & 0.6 & 0.7 & 0.5 & 0.6 & 0.6 & 0.5 \\
\hline 399-1-17A & $\mathrm{TU}$ & 0.0 & $\mathrm{ND}$ & 0.5 & 0.6 & 0.5 & 0.4 & 2.0 & 0.4 & 0.3 & 0.5 & 0.5 & 0.4 & 0.3 \\
\hline 399-1-18A & $\mathrm{TU}$ & ND & ND & ND & ND & ND & ND & ND & $\mathrm{ND}$ & ND & ND & & ND & ND \\
\hline 399-1-19 & $\mathrm{TU}$ & & & 0.2 & 1.4 & & (a) & (a) & (a) & (a) & (a) & (a) & (a) & (a) \\
\hline 399-1-21A & $\mathrm{TU}$ & 3.0 & 2.4 & 1.3 & 0.6 & & 0.8 & 3.0 & 0.9 & 1.2 & 1.4 & 1.0 & 0.4 & 0.6 \\
\hline 399-2-1 & $\mathrm{TU}$ & 3.0 & 1.7 & 0.9 & 0.7 & 1.2 & 1.0 & $\mathrm{ND}$ & 2.0 & & 1.4 & 2.1 & 1.0 & 1.0 \\
\hline 399-2-2 & $\mathrm{TU}$ & 5.0 & 2.5 & 7.0 & 2.3 & 2.0 & 1.9 & 4.0 & 3.0 & 5.3 & 2.7 & 4.2 & 1.5 & 1.8 \\
\hline 399-2-3 & $\mathrm{TU}$ & $\mathrm{ND}$ & 1.1 & 3.3 & 0.4 & $\mathrm{ND}$ & & & & & & & & \\
\hline 399-3-1 & $\mathrm{TU}$ & & & 2.3 & 0.9 & $\mathrm{ND}$ & & & & & & & & \\
\hline 399-3-2 & $\mathrm{TU}$ & 6.0 & 2.0 & 3.2 & 1.8 & & 0.7 & 0.8 & 1.0 & 0.6 & & 1.4 & 3.7 & 1.6 \\
\hline 399-3-3 & $\mathrm{TU}$ & 6.0 & & 3.9 & & & 4.0 & & 2.0 & 2.7 & & & & \\
\hline 399-3-6 & $\mathrm{TU}$ & & & 2.9 & 1.5 & 1.1 & 0.8 & 1.0 & 1.0 & 0.9 & & 2.1 & 1.4 & 1.2 \\
\hline 399-3-9 & $\mathrm{TU}$ & 1.0 & & 1.9 & & & & & & & & & & \\
\hline 399-3-10 & $\mathrm{TU}$ & 2.0 & 1.4 & 0.7 & 0.6 & 0.6 & 1.0 & 2.0 & 1.0 & 1.1 & 1.1 & 1.4 & 1.1 & 0.5 \\
\hline 399-3-11 & $\mathrm{TU}$ & 4.0 & 3.0 & 3.1 & 1.3 & 1.2 & 2.0 & 2.0 & 1.0 & 1.5 & 2.2 & 1.4 & 2.1 & 1.8 \\
\hline 399-3-12 & $\mathrm{TU}$ & 6.0 & 4.0 & 6.1 & & & & & & & & 1.4 & 2.0 & 1.4 \\
\hline 399-4-1 & $\mathrm{TU}$ & 7.0 & 4.0 & 4.4 & 3.2 & 2.4 & 3.4 & 2.0 & 5.0 & 2.3 & 2.5 & 4.3 & 2.7 & 2.1 \\
\hline 399-4-7 & $\mathrm{TU}$ & 4.0 & 5.0 & 2.0 & 3.1 & 1.4 & & & & 1.8 & 2.5 & & & \\
\hline 399-4-9 & $\mathrm{TU}$ & 3.0 & 2.2 & 1.7 & & & & & 1.0 & 1.2 & 1.1 & 0.5 & 1.2 & 1.1 \\
\hline 399-4-10 & $\mathrm{TU}$ & 3.0 & 2.7 & 1.2 & 0.6 & & & & 2.0 & 1.3 & 1.2 & 1.1 & & 0.8 \\
\hline 399-4-11 & $\mathrm{TU}$ & 4.0 & 3.0 & 3.3 & ND & 0.7 & & & 1.0 & 1.7 & 1.9 & 1.9 & 1.9 & \\
\hline 399-4-12 & $\mathrm{TU}$ & 7.0 & 6.0 & 7.0 & 5.0 & 3.0 & 3.6 & 3.0 & 4.0 & 2.7 & 3.3 & 3.8 & 3.5 & 3.7 \\
\hline 399-5-1 & $\mathrm{TU}$ & $\mathrm{ND}$ & 0.7 & 2.6 & 0.7 & 1.2 & 5.0 & 4.0 & 3.0 & 1.8 & 2.1 & & & \\
\hline 399-5-4B & $\mathrm{TU}$ & & & & 0.9 & 0.7 & 0.8 & ND & 0.8 & 0.8 & 1.0 & 1.1 & 1.0 & 0.9 \\
\hline 399-6-1 & $\mathrm{TU}$ & 2.0 & 2.0 & & 0.2 & $\mathrm{ND}$ & 0.5 & 0.8 & 0.6 & 0.6 & & & & \\
\hline 399-6-2 & $\mathrm{TU}$ & & & & 1.6 & 0.8 & 0.8 & 1.0 & 1.0 & 0.7 & 1.4 & 1.4 & 1.5 & \\
\hline 399-8-1 & $\mathrm{TU}$ & $\mathrm{ND}$ & 2.0 & 1.7 & 1.1 & $\mathrm{ND}$ & 0.8 & 0.7 & 0.6 & 0.5 & 0.7 & & & \\
\hline 399-8-2 & $\mathrm{TU}$ & ND & & & & & & & & & & & & \\
\hline 399-8-3 & $\mathrm{TU}$ & 1.0 & $\mathrm{ND}$ & & & & & & & & & & & \\
\hline 399-8-4 & $\mathrm{TU}$ & $\mathrm{ND}$ & & & & & & & & & & & & \\
\hline 399-8-5A & $\mathrm{TU}$ & 2.0 & 2.0 & 1.6 & & 0.7 & 0.7 & ND & 0.6 & 0.4 & 0.5 & 0.6 & 0.5 & 1.4 \\
\hline 699-S19-E13 & $\mathrm{TU}$ & $\mathrm{ND}$ & & & ND & & & & & & & & & \\
\hline 699-S19-E14 & $\mathrm{TU}$ & $\mathrm{ND}$ & $\mathrm{ND}$ & & & & & & & & & & & \\
\hline 699-S27-E12A & $\mathrm{TU}$ & & & & & 6.0 & 5.0 & 6.0 & 3.0 & 2.5 & 2.6 & 2.1 & 1.9 & 1.6 \\
\hline 699-S27-E14 & $\mathrm{TU}$ & 0.8 & 1.2 & 1.4 & 0.2 & 0.5 & 1.0 & 2.0 & 0.8 & 1.1 & 0.9 & 1.2 & 0.8 & 0.6 \\
\hline
\end{tabular}


Table 2.6. (contd)

\begin{tabular}{|c|c|c|c|c|c|c|c|c|c|c|c|c|c|c|}
\hline Well Name & Unit & 1992 & 1993 & 1994 & 1995 & 1996 & 1997 & 1998 & 1999 & 2000 & 2001 & 2002 & 2003 & 2004 \\
\hline 699-S28-E13A & TU & & & & & ND & 1.0 & 2.0 & 0.8 & 0.9 & 0.9 & 0.5 & 0.6 & 0.4 \\
\hline 699-S29-E12 & TU & 0.4 & ND & ND & 28.0 & ND & 0.8 & 2.0 & 1.0 & & & ND & 0.2 & 0.4 \\
\hline 699-S29-E13A & TU & & & & & ND & ND & ND & ND & ND & ND & ND & ND & ND \\
\hline 699-S29-E16A & $\mathrm{TU}$ & ND & ND & ND & ND & 0.1 & ND & ND & & ND & ND & $\mathrm{ND}$ & ND & ND \\
\hline 699-S30-E15A & $\mathrm{TU}$ & 0.1 & & & & & & & & & & & & \\
\hline \multicolumn{15}{|c|}{ Aquifer tubes at the shoreline that monitor the top of the unconfined aquifer } \\
\hline AT-3-1-D(1) & TU-S & & & & & & & & & & & & & $\mathrm{ND}$ \\
\hline AT-3-2-M & TU-S & & & & & & & & & & & & & 0.3 \\
\hline AT-3-3-M & TU-S & & & & & & & & & & & & & 6.8 \\
\hline AT-3-4-S & TU-S & & & & & & & & & & & & & 0.7 \\
\hline AT-3-5-S & TU-S & & & & & & & & & & & & & 0.5 \\
\hline AT-3-6-S & TU-S & & & & & & & & & & & & & 1.3 \\
\hline AT-3-7-M & TU-S & & & & & & & & & & & & & 2.1 \\
\hline AT-3-8-S & TU-S & & & & & & & & & & & & & 1.2 \\
\hline \multicolumn{15}{|c|}{ Wells that monitor the bottom of the unconfined aquifer } \\
\hline 399-1-8 & BU & 2.0 & 3.0 & 1.5 & & & & & & & ND & $\mathrm{ND}$ & 4.0 & 0.2 \\
\hline 399-1-10B & $\mathrm{BU}$ & ND & ND & ND & ND & ND & ND & ND & ND & ND & ND & ND & ND & ND \\
\hline 399-1-13B & $\mathrm{BU}$ & ND & ND & ND & & & & & & & & & & \\
\hline 399-1-14B & $\mathrm{BU}$ & ND & ND & ND & ND & & ND & & & & & & & \\
\hline 399-1-16B & $\mathrm{BU}$ & 16.0 & 11.0 & 5.4 & 6.0 & 2.8 & 10.0 & 8.0 & 6.0 & 3.5 & 3.1 & 2.8 & 3.3 & 2.8 \\
\hline 399-1-17B & $\mathrm{BU}$ & ND & $\mathrm{ND}$ & ND & ND & ND & 0.0 & ND & ND & ND & ND & ND & ND & ND \\
\hline 399-1-18B & $\mathrm{BU}$ & ND & & & & ND & $\mathrm{ND}$ & ND & ND & ND & ND & & $\mathrm{ND}$ & $\mathrm{ND}$ \\
\hline 399-1-21B & $\mathrm{BU}$ & ND & ND & ND & 0.4 & & & & & & ND & $\mathrm{ND}$ & ND & 0.4 \\
\hline 699-S29-E16B & $\mathrm{BU}$ & ND & $\mathrm{ND}$ & ND & ND & & & & & & & & & \\
\hline \multicolumn{15}{|c|}{ Wells that monitor the uppermost confined aquifer } \\
\hline 399-1-9 & $\mathrm{C}$ & ND & ND & ND & & & & & & & & & & \\
\hline 399-1-16C & $\mathrm{C}$ & 22.0 & ND & ND & ND & ND & & & & & & & & \\
\hline 399-1-17C & $\mathrm{C}$ & ND & & & & & & & & & & & & \\
\hline $399-1-18 C$ & $\mathrm{C}$ & ND & & & & & & & & & & & & \\
\hline 399-8-5B & $\mathrm{C}$ & ND & ND & ND & ND & & & & & & & & & \\
\hline 399-8-5C & $\mathrm{C}$ & ND & ND & ND & ND & & & & & & & & & \\
\hline 699-S29-E16C & $\mathrm{C}$ & ND & ND & ND & ND & & & & & & & & & \\
\hline \multicolumn{15}{|c|}{$\begin{array}{l}\text { (a) Well out-of-service. } \\
\text { Note: Maximum values for trichloroethene at } 300 \text { Area wells. Values in bold in shaded cells are below the EPA drinking water standard (5 } \\
\mu \mathrm{g} / \mathrm{L}) . \text { Blanks indicate "no results." } \\
\mathrm{BU}=\text { Bottom of unconfined aquifer. } \\
\mathrm{C}=\text { First confined aquifer. } \\
\mathrm{ND}=\text { Not detected. } \\
\mathrm{TU}=\text { Top of unconfined aquifer. } \\
\text { TU-S= Top of unconfined aquifer at shoreline. }\end{array}$} \\
\hline
\end{tabular}


Table 2.7. Maximum Tetrachloroethene Concentrations $(\mu \mathrm{g} / \mathrm{L})$ at 300 Area Wells

\begin{tabular}{|c|c|c|c|c|c|c|c|c|c|c|c|c|c|c|}
\hline Well Name & Unit & 1992 & 1993 & 1994 & 1995 & 1996 & 1997 & 1998 & 1999 & 2000 & 2001 & 2002 & 2003 & 2004 \\
\hline \multicolumn{15}{|c|}{ Wells that monitor the top of the unconfined aquifer } \\
\hline $399-1-1$ & $\mathrm{TU}$ & & & & & & & & & & & ND & ND & ND \\
\hline $399-1-2$ & $\mathrm{TU}$ & & 0.2 & 0.6 & ND & & & & & & & ND & ND & ND \\
\hline $399-1-3$ & $\mathrm{TU}$ & & & & ND & & & 16.0 & 0.9 & ND & ND & & & \\
\hline $399-1-4$ & $\mathrm{TU}$ & & ND & & 0.8 & & & & & & & & & \\
\hline $399-1-5$ & $\mathrm{TU}$ & ND & ND & ND & & & & & & (a) & (a) & (a) & (a) & (a) \\
\hline $399-1-6$ & $\mathrm{TU}$ & ND & ND & ND & ND & & & & & & & & ND & ND \\
\hline 399-1-7 & $\mathrm{TU}$ & ND & ND & ND & & & & & & & ND & $\mathrm{ND}$ & 0.2 & 0.2 \\
\hline 399-1-10A & $\mathrm{TU}$ & ND & ND & 0.8 & 0.1 & 0.2 & 0.2 & 8.0 & 1.0 & ND & ND & ND & ND & ND \\
\hline 399-1-11 & $\mathrm{TU}$ & ND & 0.6 & 0.6 & 0.1 & 0.9 & & & & & ND & $\mathrm{ND}$ & ND & ND \\
\hline 399-1-12 & $\mathrm{TU}$ & ND & 0.5 & 0.7 & 0.2 & 0.8 & & & & & & ND & ND & ND \\
\hline 399-1-13A & $\mathrm{TU}$ & ND & ND & & & & & & & & & & & \\
\hline 399-1-14A & $\mathrm{TU}$ & ND & 0.3 & 0.7 & 0.4 & ND & 0.5 & 0.9 & 0.9 & ND & ND & & & \\
\hline 399-1-15 & $\mathrm{TU}$ & ND & ND & & & & & & & & & ND & ND & ND \\
\hline 399-1-16A & $\mathrm{TU}$ & ND & 0.6 & 0.6 & 0.5 & 1.6 & 1.0 & 17.0 & 4.0 & ND & ND & ND & ND & ND \\
\hline 399-1-17A & $\mathrm{TU}$ & 0.0 & ND & 0.6 & 0.6 & 0.9 & 1.2 & 38.0 & 4.0 & 0.6 & ND & ND & ND & 0.1 \\
\hline 399-1-18A & $\mathrm{TU}$ & ND & $\mathrm{ND}$ & $\mathrm{ND}$ & ND & ND & ND & ND & ND & ND & ND & & ND & ND \\
\hline 399-1-19 & $\mathrm{TU}$ & & & 0.1 & 0.2 & & (a) & (a) & (a) & (a) & (a) & (a) & (a) & (a) \\
\hline 399-1-21A & $\mathrm{TU}$ & ND & 0.2 & 0.3 & ND & & ND & 1.0 & ND & ND & ND & ND & ND & ND \\
\hline 399-2-1 & $\mathrm{TU}$ & ND & ND & 0.7 & 0.1 & ND & 0.3 & 7.0 & 0.9 & & ND & $\mathrm{ND}$ & ND & ND \\
\hline $399-2-2$ & $\mathrm{TU}$ & ND & 0.3 & 0.5 & 0.5 & ND & ND & 18.0 & 1.0 & ND & ND & ND & ND & ND \\
\hline 399-2-3 & $\mathrm{TU}$ & ND & ND & 0.4 & 0.1 & ND & & & & & & & & \\
\hline 399-3-1 & $\mathrm{TU}$ & & & 0.3 & ND & $\mathrm{ND}$ & & & & & & & & \\
\hline 399-3-2 & $\mathrm{TU}$ & ND & ND & 0.5 & 0.3 & & ND & ND & ND & ND & & $\mathrm{ND}$ & 0.4 & 0.2 \\
\hline 399-3-3 & $\mathrm{TU}$ & ND & & 0.4 & & 0.3 & 0.3 & & ND & ND & & & & \\
\hline 399-3-6 & $\mathrm{TU}$ & & & 0.5 & 0.1 & 0.1 & ND & 0.2 & ND & ND & & 0.4 & 0.6 & 0.4 \\
\hline 399-3-9 & $\mathrm{TU}$ & ND & & 0.2 & & & & & & & & & & \\
\hline 399-3-10 & $\mathrm{TU}$ & ND & ND & 0.5 & ND & ND & 0.5 & 3.0 & 0.7 & ND & ND & $\mathrm{ND}$ & ND & ND \\
\hline 399-3-11 & $\mathrm{TU}$ & ND & ND & 0.3 & 0.1 & 0.1 & ND & 0.4 & ND & ND & ND & ND & 0.3 & ND \\
\hline 399-3-12 & $\mathrm{TU}$ & ND & 0.2 & 0.3 & & & & & & & & ND & 0.3 & ND \\
\hline 399-4-1 & $\mathrm{TU}$ & ND & ND & 0.8 & 0.2 & 0.2 & 0.4 & 0.5 & 0.4 & ND & 0.4 & 0.4 & 0.5 & 0.4 \\
\hline \begin{tabular}{|l|}
$399-4-7$ \\
\end{tabular} & $\mathrm{TU}$ & ND & ND & ND & ND & 0.2 & & & & ND & ND & & & \\
\hline 399-4-9 & $\mathrm{TU}$ & ND & 0.3 & 0.2 & & & & & 1.0 & ND & ND & ND & 0.2 & ND \\
\hline 399-4-10 & $\mathrm{TU}$ & ND & 0.3 & ND & ND & & & & 1.0 & ND & ND & ND & & ND \\
\hline 399-4-11 & $\mathrm{TU}$ & ND & ND & 0.4 & ND & ND & & & ND & ND & ND & 0.4 & 0.6 & \\
\hline $399-4-12$ & $\mathrm{TU}$ & ND & ND & ND & 0.2 & & 0.3 & 0.4 & 0.4 & ND & ND & ND & 0.5 & 0.3 \\
\hline $399-5-1$ & $\mathrm{TU}$ & ND & ND & $\mathrm{ND}$ & ND & 0.2 & ND & ND & ND & ND & ND & & & \\
\hline $399-5-4 B$ & $\mathrm{TU}$ & & & & ND & 0.3 & 0.2 & ND & ND & ND & ND & ND & 0.2 & ND \\
\hline 399-6-1 & $\mathrm{TU}$ & ND & $\mathrm{ND}$ & & ND & ND & ND & $\mathrm{ND}$ & ND & ND & & & & \\
\hline $399-6-2$ & $\mathrm{TU}$ & & & & ND & 0.3 & 0.1 & $\mathrm{ND}$ & ND & ND & ND & $\mathrm{ND}$ & 0.2 & \\
\hline 399-8-1 & $\mathrm{TU}$ & ND & ND & ND & ND & ND & ND & ND & ND & ND & ND & & & \\
\hline $399-8-2$ & $\mathrm{TU}$ & 4.0 & & & & & & & & & & & & \\
\hline 399-8-3 & $\mathrm{TU}$ & ND & ND & & & & & & & & & & & \\
\hline 399-8-4 & $\mathrm{TU}$ & 2.0 & & & & & & & & & & & & \\
\hline 399-8-5A & $\mathrm{TU}$ & ND & 0.2 & 0.2 & & ND & ND & 0.2 & ND & ND & ND & ND & 0.3 & 0.5 \\
\hline 699-S19-E13 & $\mathrm{TU}$ & ND & & & ND & & & & & & & & & \\
\hline 699-S19-E14 & $\mathrm{TU}$ & ND & ND & & & & & & & & & & & \\
\hline 699-S27-E12A & $\mathrm{TU}$ & & & & & ND & $\mathrm{ND}$ & ND & ND & ND & ND & $\mathrm{ND}$ & ND & ND \\
\hline 699-S27-E14 & $\mathrm{TU}$ & ND & ND & ND & ND & 0.1 & ND & ND & ND & ND & ND & $\mathrm{ND}$ & ND & ND \\
\hline
\end{tabular}


Table 2.7. (contd)

\begin{tabular}{|c|c|c|c|c|c|c|c|c|c|c|c|c|c|c|}
\hline Well Name & Unit & 1992 & 1993 & 1994 & 1995 & 1996 & 1997 & 1998 & 1999 & 2000 & 2001 & 2002 & 2003 & 2004 \\
\hline 699-S28-E13A & $\mathrm{TU}$ & & & & & ND & ND & $\mathrm{ND}$ & ND & ND & ND & ND & $\mathrm{ND}$ & ND \\
\hline 699-S29-E12 & TU & ND & ND & ND & ND & ND & ND & 0.5 & ND & & & ND & ND & ND \\
\hline 699-S29-E13A & TU & & & & & ND & ND & ND & ND & ND & ND & ND & ND & ND \\
\hline 699-S29-E16A & $\mathrm{TU}$ & ND & ND & $\mathrm{ND}$ & ND & 0.2 & ND & ND & & ND & ND & ND & ND & ND \\
\hline 699-S30-E15A & $\mathrm{TU}$ & 0.2 & & & & & & & & & & & & \\
\hline \multicolumn{15}{|c|}{ Aquifer tubes at the shoreline that monitor the top of the unconfined aquifer } \\
\hline AT-3-1-D(1) & TU-S & & & & & & & & & & & & & ND \\
\hline AT-3-2-M & TU-S & & & & & & & & & & & & & ND \\
\hline AT-3-3-M & TU-S & & & & & & & & & & & & & ND \\
\hline AT-3-4-S & TU-S & & & & & & & & & & & & & ND \\
\hline AT-3-5-S & TU-S & & & & & & & & & & & & & ND \\
\hline AT-3-6-S & TU-S & & & & & & & & & & & & & ND \\
\hline AT-3-7-M & TU-S & & & & & & & & & & & & & ND \\
\hline AT-3-8-S & TU-S & & & & & & & & & & & & & ND \\
\hline \multicolumn{15}{|c|}{ Wells that monitor the bottom of the unconfined aquifer } \\
\hline 399-1-8 & $\mathrm{BU}$ & $\mathrm{ND}$ & 0.3 & 0.3 & & & & & & & ND & ND & $\mathrm{ND}$ & ND \\
\hline 399-1-10B & $\mathrm{BU}$ & ND & ND & $\mathrm{ND}$ & ND & ND & $\mathrm{ND}$ & ND & ND & ND & ND & $\mathrm{ND}$ & ND & ND \\
\hline 399-1-13B & $\mathrm{BU}$ & $\mathrm{ND}$ & $\mathrm{ND}$ & ND & & & & & & & & & & \\
\hline 399-1-14B & $\mathrm{BU}$ & ND & ND & ND & ND & & ND & & & & & & & \\
\hline 399-1-16B & $\mathrm{BU}$ & 0.4 & $\mathrm{ND}$ & 0.4 & ND & ND & ND & 1.0 & 0.4 & ND & ND & ND & ND & ND \\
\hline 399-1-17B & $\mathrm{BU}$ & $\mathrm{ND}$ & ND & ND & ND & 0.2 & ND & ND & ND & ND & ND & ND & ND & ND \\
\hline 399-1-18B & $\mathrm{BU}$ & ND & & & & ND & ND & ND & ND & ND & ND & & ND & ND \\
\hline 399-1-21B & $\mathrm{BU}$ & ND & ND & ND & ND & & & & & & ND & ND & ND & ND \\
\hline 699-S29-E16B & $\mathrm{BU}$ & ND & ND & ND & ND & & & & & & & & & \\
\hline \multicolumn{15}{|c|}{ Wells that monitor the uppermost confined aquifer } \\
\hline 399-1-9 & $\mathrm{C}$ & $\mathrm{ND}$ & ND & ND & & & & & & & & & & \\
\hline $399-1-16 \mathrm{C}$ & $\mathrm{C}$ & ND & ND & ND & ND & ND & & & & & & & & \\
\hline $399-1-17 \mathrm{C}$ & $\mathrm{C}$ & $\mathrm{ND}$ & & & & & & & & & & & & \\
\hline $399-1-18 \mathrm{C}$ & $\mathrm{C}$ & $\mathrm{ND}$ & & & & & & & & & & & & \\
\hline 399-8-5B & $\mathrm{C}$ & ND & ND & ND & ND & & & & & & & & & \\
\hline $399-8-5 \mathrm{C}$ & $\mathrm{C}$ & ND & ND & ND & ND & & & & & & & & & \\
\hline 699-S29-E16C & $\mathrm{C}$ & ND & ND & ND & ND & & & & & & & & & \\
\hline \multicolumn{15}{|c|}{$\begin{array}{l}\text { (a) Well out-of-service. } \\
\text { Note: Maximum values for tetrachloroethene at } 300 \text { Area wells. Values in bold in shaded cells are below the EPA drinking water standard } \\
(5 \mu \mathrm{g} / \mathrm{L}) . \text { Blanks indicate "no results." } \\
\mathrm{BU}=\text { Bottom of unconfined aquifer. } \\
\mathrm{C} \quad=\text { First confined aquifer. } \\
\text { EPA }=\text { U.S. Environmental Protection Agency. } \\
\text { ND }=\text { Not detected. } \\
\text { TU }=\text { Top of unconfined aquifer. } \\
\text { TU-S }=\text { Top of unconfined aquifer at shoreline. }\end{array}$} \\
\hline
\end{tabular}


Table 2.8. Maximum Strontium-90 Concentrations (pCi/L) at 300 Area Wells

\begin{tabular}{|c|c|c|c|c|c|c|c|c|c|c|c|c|c|c|}
\hline "Well Name & "Unit & 1992 & 1993 & 1994 & 1995 & 1996 & 1997 & 1998 & 1999 & 2000 & 2001 & 2002 & 2003 & 2004 \\
\hline \multicolumn{15}{|c|}{ Wells that monitor the top of the unconfined aquifer } \\
\hline $399-1-5$ & $\mathrm{TU}$ & 1.0 & & & & & & & & (a) & (a) & (a) & (a) & (a) \\
\hline $399-1-6$ & TU & ND & & & & & & & & & & & & \\
\hline $399-1-7$ & TU & 0.7 & & & & & & & & & & & & \\
\hline 399-1-10A & TU & ND & & & & & & & & & & & & \\
\hline $399-1-11$ & TU & 2.0 & & & & & & & & & & & & \\
\hline 399-1-13A & TU & ND & & & & & & & & & & & & \\
\hline 399-1-14A & $\mathrm{TU}$ & ND & & & & & & & & & & & & \\
\hline $399-1-15$ & TU & ND & & & & & & & & & & & & \\
\hline 399-1-16A & $\mathrm{TU}$ & ND & & & & & & & & & & & & \\
\hline $399-1-17 A$ & $\mathrm{TU}$ & 1.3 & 2.1 & 0.9 & 1.6 & 0.8 & & & & & & & & \\
\hline 399-1-18A & TU & ND & & & & & & & & & & & & \\
\hline $399-1-21 \mathrm{~A}$ & $\mathrm{TU}$ & ND & & & & & & & & & & & & \\
\hline $399-2-1$ & $\mathrm{TU}$ & ND & & & & & & & & & & & & \\
\hline $399-2-2$ & $\mathrm{TU}$ & ND & & & & & & & & & & & & \\
\hline $399-2-3$ & $\mathrm{TU}$ & ND & & & & & & & & & & & & \\
\hline $399-3-2$ & $\mathrm{TU}$ & ND & & & & & & & & & & & & \\
\hline 399-3-3 & $\mathrm{TU}$ & 4.6 & & & & & & & & & & & & \\
\hline 399-3-11 & $\mathrm{TU}$ & ND & & ND & 8.7 & 3.0 & 3.1 & 3.6 & 4.1 & 4.1 & 3.6 & 3.4 & 4.0 & 3.4 \\
\hline 399-3-12 & $\mathrm{TU}$ & ND & & & & & & & & & & & & \\
\hline $399-4-1$ & $\mathrm{TU}$ & ND & & & & & & & & & & & & \\
\hline $399-4-9$ & $\mathrm{TU}$ & ND & & & & & & & & & & & & \\
\hline $399-4-10$ & $\mathrm{TU}$ & 0.3 & & & & & & & & & & & & \\
\hline $399-4-11$ & TU & ND & & 0.2 & & 0.0 & & & & & & & & \\
\hline $399-4-12$ & $\mathrm{TU}$ & ND & & & & & & & & & & & & \\
\hline 399-5-1 & $\mathrm{TU}$ & ND & & & & & & & & & & & & \\
\hline 399-6-1 & TU & ND & & & & & & & & & & & & \\
\hline 399-8-1 & TU & ND & & & & & & & & & & & & \\
\hline 399-8-2 & $\mathrm{TU}$ & ND & & & & & & & & & & & & \\
\hline 399-8-3 & $\mathrm{TU}$ & ND & & & & & & & & & & & & \\
\hline 399-8-4 & TU & ND & & & & & & & & & & & & \\
\hline $399-8-5 A$ & TU & ND & & & & & & & & & & & & \\
\hline 699-S19-E13 & $\mathrm{TU}$ & & & & & & & & ND & ND & ND & & ND & $\mathrm{ND}$ \\
\hline 699-S19-E14 & $\mathrm{TU}$ & ND & & & & & & & & & & & & \\
\hline 699-S29-E16A & $\mathrm{TU}$ & ND & & & & & & & & & & & & \\
\hline 699-S30-E15A & $\mathrm{TU}$ & ND & & & & & & & & & & & & \\
\hline \multicolumn{15}{|c|}{ Wells that monitor the bottom of the unconfined aquifer } \\
\hline $399-1-8$ & BU & 4.1 & & & & & & & & & & & & \\
\hline 399-1-10B & $\mathrm{BU}$ & ND & & & & & & & & & & & & \\
\hline 399-1-13B & $\mathrm{BU}$ & ND & & & & & & & & & & & & \\
\hline 399-1-14B & BU & ND & & & & & & & & & & & & \\
\hline 399-1-16B & $\mathrm{BU}$ & ND & & & & & & & & & & & & \\
\hline 399-1-18B & $\mathrm{BU}$ & ND & & & & & & & & & & & & \\
\hline 399-1-21B & $\mathrm{BU}$ & ND & & & & & & & & & & & & \\
\hline 699-S29-E16B & $\mathrm{BU}$ & ND & & & & & & & & & & & & \\
\hline
\end{tabular}


Table 2.8. (contd)

\begin{tabular}{|c|c|c|c|c|c|c|c|c|c|c|c|c|c|c|}
\hline Well Name & Unit & 1992 & 1993 & 1994 & 1995 & 1996 & 1997 & 1998 & 1999 & 2000 & 2001 & 2002 & 2003 & 2004 \\
\hline \multicolumn{15}{|c|}{ Wells that monitor the uppermost confined aquifer } \\
\hline $399-1-9$ & $\mathrm{C}$ & ND & & & & & & & & & & & & \\
\hline $399-1-16 C$ & $\mathrm{C}$ & ND & & & & & & & & & & & & \\
\hline $399-1-17 \mathrm{C}$ & $\mathrm{C}$ & ND & & & & & & & & & & & & \\
\hline $399-1-18 \mathrm{C}$ & $\mathrm{C}$ & ND & & & & & & & & & & & & \\
\hline 399-8-5B & C & ND & & & & & & & & & & & & \\
\hline $399-8-5 \mathrm{C}$ & $\mathrm{C}$ & ND & & & & & & & & & & & & \\
\hline 699-S29-E16C & $\mathrm{C}$ & ND & & & & & & & & & & & & \\
\hline $\begin{array}{l}\text { (a) Well out-o } \\
\text { Note: Maximu } \\
\mathrm{pCi} / \mathrm{L}) \text {. Blanks } \\
\mathrm{BU}=\text { Botton } \\
\mathrm{C}=\text { First c } \\
\mathrm{EPA}=\text { U.S. } \\
\mathrm{ND}=\text { Not de } \\
\mathrm{TU}=\text { Top of } \\
\mathrm{TU}-\mathrm{S}=\text { Top of }\end{array}$ & $\begin{array}{l}\text { vice. } \\
\text { lues fo } \\
\text { ate "nc } \\
\text { nconfi } \\
\text { ed aqu } \\
\text { nment } \\
\text { nfined } \\
\text { nfined }\end{array}$ & $\begin{array}{l}\text { stronti, } \\
\text { esults.' } \\
\text { ed aqui } \\
\text { er. } \\
\text { Protect } \\
\text { quifer. } \\
\text { quifer }\end{array}$ & n-90 at & 00 Are & wells. & Values i & bold ir & shaded & ells are & elow tl & EPA & inking & ater sta & dard (8 \\
\hline
\end{tabular}


Table 2.9. Maximum Tritium Concentrations (pCi/L) at 300 Area Wells

\begin{tabular}{|c|c|c|c|c|c|c|c|c|c|c|c|c|c|c|}
\hline Well Name & Unit & 1992 & 1993 & 1994 & 1995 & 1996 & 1997 & 1998 & 1999 & 2000 & 2001 & 2002 & 2003 & 2004 \\
\hline \multicolumn{15}{|c|}{ Wells that monitor the top of the unconfined aquifer } \\
\hline 399-1-1 & $\mathrm{TU}$ & & & & & 1,218 & & & & & & & & \\
\hline $399-1-2$ & $\mathrm{TU}$ & & 6,950 & 10,000 & 11,200 & 11,243 & & 11,200 & & & & & & \\
\hline $399-1-3$ & TU & & & & 1,080 & & & 12,100 & & & & & & \\
\hline 399-1-4 & $\mathrm{TU}$ & & 10,600 & & 11,775 & & & & & & & & & \\
\hline 399-1-5 & $\mathrm{TU}$ & 6,904 & & & & & & & & (a) & (a) & (a) & (a) & (a) \\
\hline 399-1-6 & $\mathrm{TU}$ & & 10,600 & 11,300 & 12,700 & & & & & & & & & \\
\hline $399-1-7$ & $\mathrm{TU}$ & 2,290 & & & & & & & & & & & & \\
\hline 399-1-10A & $\mathrm{TU}$ & 10,000 & 10,200 & 10,800 & 12,149 & 657 & 10,800 & 12,300 & & & & & & \\
\hline 399-1-11 & $\mathrm{TU}$ & 10,300 & 10,500 & 10,900 & 12,700 & 12,525 & 11,000 & & & & & & & \\
\hline $399-1-12$ & $\mathrm{TU}$ & 9,030 & 9,630 & 9,640 & 12,100 & 11,497 & 10,900 & 12,300 & & & & & & \\
\hline 399-1-13A & $\mathrm{TU}$ & 8,270 & & 9,980 & 11,500 & 11,271 & & & 12,900 & 8,700 & 4,780 & & & \\
\hline 399-1-14A & $\mathrm{TU}$ & 10,000 & 10,000 & 10,700 & 12,600 & 13,501 & 11,600 & 11,900 & 12,800 & 11,600 & 8,190 & & & \\
\hline 399-1-15 & $\mathrm{TU}$ & 10,300 & & 11,900 & & & & & & & & & & \\
\hline 399-1-16A & $\mathrm{TU}$ & 8,240 & 8,920 & 9,160 & 12,400 & 2,255 & 12,300 & 11,600 & & & & & & \\
\hline 399-1-17A & $\mathrm{TU}$ & 373 & 702 & 1,940 & 11,800 & 10,952 & 12,300 & 11,300 & 11,900 & 11,600 & 8,870 & 8,910 & 9,750 & 9,090 \\
\hline 399-1-18A & $\mathrm{TU}$ & 11,300 & 11,100 & 11,500 & 13,801 & 12,672 & 14,100 & 13,300 & & & & & & \\
\hline 399-1-19 & $\mathrm{TU}$ & & & 473 & 10,297 & & (a) & (a) & (a) & (a) & (a) & (a) & (a) & (a) \\
\hline $399-1-21 \mathrm{~A}$ & $\mathrm{TU}$ & 4,130 & 4,560 & 3,970 & 3,151 & 7,671 & 8,960 & 9,170 & 9,650 & 8,420 & 2,070 & 7,280 & 6,410 & 3,460 \\
\hline $399-2-1$ & $\mathrm{TU}$ & 2,390 & 6,500 & 4,510 & 9,159 & 385 & 8,190 & 11,400 & & & & & & \\
\hline $399-2-2$ & $\mathrm{TU}$ & & 3,890 & 5,710 & 12,200 & & & & & & & & & \\
\hline $399-2-3$ & $\mathrm{TU}$ & & 4,980 & 5,210 & 7,115 & 523 & & & & & & & & \\
\hline 399-3-1 & $\mathrm{TU}$ & & & 3,250 & 8,035 & $\mathbf{0}$ & & & & & & & & \\
\hline $399-3-2$ & $\mathrm{TU}$ & & & 104 & 11 & 2,570 & 870 & ND & & & & & & \\
\hline 399-3-3 & $\mathrm{TU}$ & 1,075 & & 763 & & 575 & 801 & & & & & & & \\
\hline 399-3-6 & $\mathrm{TU}$ & & & $\mathbf{0}$ & 749 & 4,300 & 2,100 & 925 & 583 & 893 & ND & 295 & 302 & ND \\
\hline 399-3-9 & $\mathrm{TU}$ & 2,370 & & 2,200 & & & & & & & & & & \\
\hline $399-3-10$ & $\mathrm{TU}$ & 2,270 & 2,650 & 3,790 & 4,510 & 686 & 5,460 & 7,560 & & & & & & \\
\hline 399-3-11 & $\mathrm{TU}$ & 4,634 & & 2,220 & 4,204 & 7,258 & 4,820 & 4,060 & 8,430 & 3,900 & ND & 3,610 & 1,240 & 637 \\
\hline $399-3-12$ & $\mathrm{TU}$ & & 3,370 & 3,320 & & 20,006 & 6,500 & 5,590 & 9,170 & & 636 & 5,040 & 2,210 & 845 \\
\hline 399-4-1 & $\mathrm{TU}$ & 921 & & 167 & 319 & 1,850 & 1,390 & 831 & 1,420 & 785 & ND & 971 & $\mathrm{ND}$ & ND \\
\hline 399-4-7 & $\mathrm{TU}$ & 3,380 & & 2,010 & 2,230 & 4,080 & 5,870 & 4,150 & 7,090 & 7,180 & 3,880 & 1,330 & 3,340 & \\
\hline 399-4-9 & $\mathrm{TU}$ & 4,200 & 3,910 & 2,490 & & & 8,790 & 9,430 & 9,710 & 9,230 & 4,590 & 548 & 5,830 & 6,700 \\
\hline $399-4-10$ & $\mathrm{TU}$ & 5,030 & 3,310 & 2,340 & 3,890 & 768 & & & & & & & & \\
\hline 399-4-11 & $\mathrm{TU}$ & 997 & & 151 & 138 & 5,090 & 2,930 & 920 & 1,860 & 420 & ND & & & \\
\hline $399-4-12$ & $\mathrm{TU}$ & 1,887 & & 145 & 254 & 3,660 & 2,640 & 805 & 2,650 & 1,000 & & 635 & 250 & 407 \\
\hline $399-5-1$ & $\mathrm{TU}$ & & 43 & $\mathbf{0}$ & $\mathbf{0}$ & 52 & 26 & 28 & 43 & 46 & 31 & & & \\
\hline 399-5-4B & $\mathrm{TU}$ & & & & & 22 & 22 & 19 & 107 & 29 & 29 & 46 & 53 & 27 \\
\hline 399-6-1 & $\mathrm{TU}$ & & & & & 43 & 55 & 28 & & & & & & \\
\hline 399-6-2 & $\mathrm{TU}$ & & & & & 28 & 26 & 24 & & & & & & \\
\hline 399-8-1 & $\mathrm{TU}$ & 34 & & 17 & ND & 3,250 & 461 & ND & ND & ND & ND & & & \\
\hline 399-8-2 & $\mathrm{TU}$ & ND & & & & & & & & & & & & \\
\hline 399-8-3 & $\mathrm{TU}$ & 412 & & 716 & 733 & 4,700 & 4,890 & 2,230 & & & & & & \\
\hline 399-8-4 & $\mathrm{TU}$ & ND & & & & & & & & & & & & \\
\hline 399-8-5A & $\mathrm{TU}$ & & $\mathbf{0}$ & 166 & & ND & 17 & 34 & 12 & 26 & 15 & 118 & 74 & 33 \\
\hline 699-S19-E13 & $\mathrm{TU}$ & 11,600 & 12,200 & 13,300 & 13,300 & 11,728 & 14,500 & 13,400 & 13,100 & 13,100 & 12,100 & 12,100 & 11,900 & 11,000 \\
\hline 699-S19-E14 & $\mathrm{TU}$ & 11,770 & 12,100 & 13,600 & 15,300 & 12,210 & 16,000 & 16,000 & & & 16,000 & & & 15,100 \\
\hline 699-S27-E12A & $\mathrm{TU}$ & & & & & & & 27 & & & & & & \\
\hline 699-S27-E14 & $\mathrm{TU}$ & 0 & 119 & 124 & 29 & 33 & 27 & 25 & 50 & 31 & 37 & 27 & 67 & 36 \\
\hline
\end{tabular}


Table 2.9. (contd)

\begin{tabular}{|c|c|c|c|c|c|c|c|c|c|c|c|c|c|c|}
\hline Well Name & Unit & 1992 & 1993 & 1994 & 1995 & 1996 & 1997 & 1998 & 1999 & 2000 & 2001 & 2002 & 2003 & 2004 \\
\hline 699-S29-E12 & TU & 74 & 206 & 105 & 3 & 24 & 21 & 30 & & & & & & \\
\hline 699-S29-E13A & TU & & & & & & & & 348 & 83 & & & & \\
\hline 699-S29-E16A & TU & & 120 & 86 & 78 & 2,030 & 69 & 81 & 71 & 86 & 73 & 76 & 93 & 394 \\
\hline 699-S30-E15A & $\mathrm{TU}$ & 191 & 293 & ND & 73 & 69 & 72 & 52 & 60 & 56 & 76 & 46 & 92 & 97 \\
\hline \multicolumn{15}{|c|}{ Aquifer tubes at the shoreline that monitor the top of the unconfined aquifer } \\
\hline 300-3-3A-410cm & TU-S & & & & & & & & & & & & & 8,840 \\
\hline 300-3-3B-376cm & TU-S & & & & & & & & & & & & & 8,340 \\
\hline 300SPR 9A-19cm & TU-S & & & & & & & & & & & & & 8,860 \\
\hline 300SPR 9A-86cm & TU-S & & & & & & & & & & & & & 10,000 \\
\hline AT-3-3-M & TU-S & & & & & & & & & & & & & 9,320 \\
\hline AT-3-3-S & TU-S & & & & & & & & & & & & & 9,340 \\
\hline \multicolumn{15}{|c|}{ Wells that monitor the bottom of the unconfined aquifer } \\
\hline $399-1-8$ & BU & & 3,590 & 2,740 & & & & & & & & & & \\
\hline 399-1-10B & $\mathrm{BU}$ & 20 & 37 & 113 & $\mathbf{0}$ & ND & ND & 78 & 13 & & 46 & ND & $\mathrm{ND}$ & ND \\
\hline 399-1-13B & $\mathrm{BU}$ & 12 & 71 & 75 & $\mathbf{0}$ & ND & & & & & & & & \\
\hline 399-1-14B & $\mathrm{BU}$ & $\mathbf{0}$ & $\mathbf{0}$ & 161 & $\mathbf{0}$ & ND & ND & & & & & & & \\
\hline 399-1-16B & $\mathrm{BU}$ & 404 & 358 & 625 & 575 & ND & 969 & 1,240 & & & & 690 & 750 & \\
\hline 399-1-17B & $\mathrm{BU}$ & 77 & ND & ND & 0 & ND & ND & ND & & & & & & \\
\hline 399-1-18B & $\mathrm{BU}$ & & & & & & & 72 & 6 & & 11 & ND & ND & ND \\
\hline 399-1-21B & $\mathrm{BU}$ & & 11 & 40 & 85 & ND & & & & & & & & \\
\hline 699-S29-E16B & $\mathrm{BU}$ & & $\mathbf{0}$ & ND & $\mathrm{ND}$ & ND & 11 & 37 & ND & 14 & ND & 10 & 1 & 14 \\
\hline \multicolumn{15}{|c|}{ Wells that monitor the uppermost confined aquifer } \\
\hline $399-1-9$ & $\mathrm{C}$ & & 27 & 44 & & & & & & & & & & \\
\hline $399-1-16 C$ & $\mathrm{C}$ & & & & $\mathbf{0}$ & & & & & & ND & ND & & 10 \\
\hline $399-1-17 C$ & $\mathrm{C}$ & & & & & ND & & & & & & & & \\
\hline $399-1-18 C$ & $\mathrm{C}$ & & & & & & & & & & 11 & 14 & 14 & \\
\hline $399-8-5 B$ & $\mathrm{C}$ & ND & $\mathbf{0}$ & 96 & $\mathbf{0}$ & & & & & & ND & & & \\
\hline $399-8-5 \mathrm{C}$ & $\mathrm{C}$ & ND & $\mathbf{0}$ & 231 & 62 & & & & & & ND & & & \\
\hline 699-S29-E16C & $\mathrm{C}$ & & $\mathbf{0}$ & ND & $\mathrm{ND}$ & ND & ND & & ND & 9 & & 13 & 2 & \\
\hline \multicolumn{15}{|c|}{$\begin{array}{l}\text { (a) Well out-of-service. } \\
\text { Note: Maximum values for tritium at } 300 \text { Area wells. Values in bold in shaded cells are below the EPA drinking water standard }(20,000 \mathrm{pCi} / \mathrm{L}) \\
\text { Blanks indicate "no results." } \\
\mathrm{BU}=\text { Bottom of unconfined aquifer. } \\
\mathrm{C} \quad=\text { First confined aquifer. } \\
\text { EPA }=\text { U.S. Environmental Protection Agency. } \\
\mathrm{ND}=\text { Not detected. } \\
\mathrm{TU}=\text { Top of unconfined aquifer. } \\
\text { TU-S }=\text { Top of unconfined aquifer at shoreline. }\end{array}$} \\
\hline
\end{tabular}


Table 2.10. Maximum Nitrate Concentrations $(\mu \mathrm{g} / \mathrm{L})$ at 300 Area Wells

\begin{tabular}{|c|c|c|c|c|c|c|c|c|c|c|c|c|c|c|}
\hline Well Name & Unit & 1992 & 1993 & 1994 & 1995 & 1996 & 1997 & 1998 & 1999 & 2000 & 2001 & 2002 & 2003 & 2004 \\
\hline \multicolumn{15}{|c|}{ Wells that monitor the top of the unconfined aquifer } \\
\hline $399-1-1$ & TU & & & & & & & & & & & 31 & 10 & 20 \\
\hline $399-1-2$ & TU & & 20 & 22 & & & & & & & & 27 & 27 & 25 \\
\hline $399-1-3$ & TU & & & & 8 & & & 29 & & & & & & \\
\hline $399-1-4$ & $\mathrm{TU}$ & & 21 & & 21 & & & & & & & & & \\
\hline $399-1-5$ & $\mathrm{TU}$ & 21 & & & & & & & & (a) & (a) & (a) & (a) & (a) \\
\hline $399-1-6$ & $\mathrm{TU}$ & 22 & 22 & 23 & 23 & & & & & & & & 30 & 30 \\
\hline 399-1-7 & $\mathrm{TU}$ & 3 & 6 & 15 & & & & & & & & & & \\
\hline 399-1-10A & $\mathrm{TU}$ & 21 & 21 & 20 & 13 & & 25 & 27 & 26 & & 27 & 32 & 19 & 26 \\
\hline 399-1-11 & $\mathrm{TU}$ & 22 & 20 & 24 & & & & & & & & & & \\
\hline 399-1-12 & $\mathrm{TU}$ & 19 & 21 & 22 & & & & & & & & & & \\
\hline 399-1-13A & $\mathrm{TU}$ & 19 & & 22 & 21 & & & & & & & & & \\
\hline 399-1-14A & $\mathrm{TU}$ & 20 & 20 & 24 & 22 & & 28 & 27 & 29 & 25 & 22 & & & \\
\hline \begin{tabular}{|l|}
$399-1-15$ \\
\end{tabular} & $\mathrm{TU}$ & 21 & 21 & 24 & & & & & & & & & & \\
\hline 399-1-16A & $\mathrm{TU}$ & 21 & 20 & & 21 & & 28 & 28 & 27 & & 26 & 26 & 10 & 26 \\
\hline 399-1-17A & $\mathrm{TU}$ & 4 & 4 & 12 & 25 & 28 & 29 & 31 & 40 & 27 & 27 & 26 & 27 & 28 \\
\hline 399-1-18A & $\mathrm{TU}$ & 22 & 23 & 25 & 23 & & 28 & 29 & 31 & & 27 & 29 & 29 & 27 \\
\hline 399-1-19 & $\mathrm{TU}$ & & & 2 & 19 & & (a) & (a) & (a) & (a) & (a) & (a) & (a) & (a) \\
\hline $399-1-21 \mathrm{~A}$ & $\mathrm{TU}$ & 17 & 21 & 12 & 22 & & 28 & 25 & 27 & 24 & 21 & 23 & & 24 \\
\hline 399-2-1 & $\mathrm{TU}$ & 10 & 17 & 11 & & & 26 & 27 & & & & & & \\
\hline $399-2-2$ & $\mathrm{TU}$ & 15 & 10 & 21 & 24 & & & & & & & & & \\
\hline 399-2-3 & $\mathrm{TU}$ & 19 & 19 & 19 & 25 & 2 & & & & & & & & \\
\hline 399-3-1 & $\mathrm{TU}$ & & & 18 & 22 & 1 & & & & & & & & \\
\hline $399-3-2$ & $\mathrm{TU}$ & 19 & & 23 & 17 & 15 & 22 & 8 & & & & & & \\
\hline 399-3-3 & $\mathrm{TU}$ & 11 & & 16 & & 23 & 29 & & & & & & & \\
\hline 399-3-6 & $\mathrm{TU}$ & & & 18 & 19 & 19 & 29 & 26 & 27 & 24 & & 27 & 25 & 28 \\
\hline 399-3-9 & $\mathrm{TU}$ & 17 & 18 & 21 & & & & & & & & & & \\
\hline 399-3-10 & $\mathrm{TU}$ & 15 & 15 & 20 & & & & & & & & & & \\
\hline 399-3-11 & $\mathrm{TU}$ & 16 & & 16 & 25 & 23 & & & 26 & 24 & & 21 & 25 & 26 \\
\hline \begin{tabular}{|l|}
$399-3-12$ \\
\end{tabular} & $\mathrm{TU}$ & 15 & 18 & 15 & & 28 & 26 & 22 & 29 & & 20 & 24 & 24 & 24 \\
\hline 399-4-1 & $\mathrm{TU}$ & 14 & 18 & 16 & 21 & 22 & & & 26 & 27 & 25 & 24 & 26 & 24 \\
\hline 399-4-7 & $\mathrm{TU}$ & 15 & 14 & 17 & 15 & & & & & & & & & \\
\hline 399-4-9 & $\mathrm{TU}$ & 17 & 16 & 17 & & & & & 35 & 26 & 20 & 5 & 24 & 22 \\
\hline \begin{tabular}{|l|}
$399-4-10$ \\
\end{tabular} & $\mathrm{TU}$ & 16 & 15 & 19 & 21 & & & & & & & & & \\
\hline 399-4-11 & $\mathrm{TU}$ & 15 & & 19 & 19 & 23 & 27 & 26 & 27 & 25 & 25 & & & \\
\hline 399-4-12 & $\mathrm{TU}$ & 16 & & 18 & 23 & 23 & 28 & 28 & 26 & 26 & 27 & 28 & 34 & 27 \\
\hline $399-5-1$ & $\mathrm{TU}$ & 66 & 81 & 92 & 57 & 35 & 113 & 99 & 112 & 100 & 89 & 90 & 97 & \\
\hline 399-5-4B & $\mathrm{TU}$ & & & & & 23 & 32 & 42 & & & & & & \\
\hline 399-6-1 & $\mathrm{TU}$ & 33 & & & & 24 & 34 & 39 & & & & & & \\
\hline 399-6-2 & $\mathrm{TU}$ & & & & & 26 & 39 & 42 & & & & & & \\
\hline 399-8-1 & $\mathrm{TU}$ & 20 & 23 & 19 & 23 & 20 & & & & & & & & \\
\hline 399-8-2 & $\mathrm{TU}$ & 15 & & & & & & & & & & & & \\
\hline 399-8-3 & $\mathrm{TU}$ & 12 & & 15 & 14 & & & & & & & & & \\
\hline 399-8-4 & $\mathrm{TU}$ & 16 & & & & & & & & & & & & \\
\hline 399-8-5A & $\mathrm{TU}$ & 24 & 26 & 25 & & 24 & 28 & 36 & 34 & 30 & 24 & 23 & 29 & 29 \\
\hline \begin{tabular}{|l|} 
699-S19-E13 \\
\end{tabular} & $\mathrm{TU}$ & 24 & 24 & 24 & 23 & 26 & 28 & 29 & 30 & 30 & 27 & 29 & 26 & 29 \\
\hline 699-S19-E14 & $\mathrm{TU}$ & 24 & 23 & 25 & 26 & & & & & & 32 & & & 35 \\
\hline 699-S27-E12A & $\mathrm{TU}$ & & & & & 141 & & 114 & 111 & 120 & 100 & 95 & 117 & 129 \\
\hline \begin{tabular}{|l|}
$699-S 27-E 14$ \\
\end{tabular} & $\mathrm{TU}$ & 22 & 25 & 21 & 28 & 41 & 63 & 58 & 70 & 61 & 55 & 62 & 63 & 70 \\
\hline
\end{tabular}


Table 2.10. (contd)

\begin{tabular}{|c|c|c|c|c|c|c|c|c|c|c|c|c|c|c|}
\hline Well Name & Unit & 1992 & 1993 & 1994 & 1995 & 1996 & 1997 & 1998 & 1999 & 2000 & 2001 & 2002 & 2003 & 2004 \\
\hline 699-S28-E13A & TU & & & & & 42 & & & 57 & 62 & 56 & 62 & 75 & 85 \\
\hline 699-S29-E12 & TU & 28 & 40 & 27 & 36 & 46 & 43 & 64 & 66 & & & 66 & 76 & 91 \\
\hline 699-S29-E13A & TU & & & & & ND & & & 44 & 40 & 47 & 66 & 75 & 76 \\
\hline 699-S29-E16A & TU & 18 & 22 & 23 & 18 & & & 40 & & 35 & 48 & 36 & 67 & 71 \\
\hline 699-S30-E15A & TU & 16 & 24 & 20 & 19 & 22 & 25 & 42 & 49 & 55 & 56 & 66 & 65 & 73 \\
\hline \multicolumn{15}{|c|}{ Aquifer tubes at the shoreline that monitor the top of the unconfined aquifer } \\
\hline AT-3-1-D(1) & TU-S & & & & & & & & & & & & & 27 \\
\hline AT-3-2-M & TU-S & & & & & & & & & & & & & 26 \\
\hline AT-3-3-M & TU-S & & & & & & & & & & & & & 27 \\
\hline AT-3-4-S & TU-S & & & & & & & & & & & & & 26 \\
\hline AT-3-5-S & TU-S & & & & & & & & & & & & & 13 \\
\hline AT-3-6-S & TU-S & & & & & & & & & & & & & 24 \\
\hline AT-3-7-M & TU-S & & & & & & & & & & & & & 54 \\
\hline AT-3-8-S & TU-S & & & & & & & & & & & & & 67 \\
\hline \multicolumn{15}{|c|}{ Wells that monitor the bottom of the unconfined aquifer } \\
\hline $399-1-8$ & $\mathrm{BU}$ & 3 & 7 & 5 & & & & & & & & & & \\
\hline 399-1-10B & $\mathrm{BU}$ & ND & ND & ND & ND & & & & & & & & & \\
\hline 399-1-13B & $\mathrm{BU}$ & ND & $\mathbf{0}$ & $\mathrm{ND}$ & ND & & & & & & & & & \\
\hline 399-1-14B & $\mathrm{BU}$ & 1 & ND & ND & ND & & & & & & & & & \\
\hline 399-1-16B & $\mathrm{BU}$ & ND & 1 & & 1 & & 2 & & & & & & & \\
\hline $399-1-17 B$ & $\mathrm{BU}$ & ND & & & $\mathbf{0}$ & & & & & & & & & \\
\hline 399-1-18B & $\mathrm{BU}$ & 1 & & & & & & & & & & & & \\
\hline $399-1-21 B$ & $\mathrm{BU}$ & 1 & 1 & ND & ND & & & & & & & & & \\
\hline 699-S29-E16B & $\mathrm{BU}$ & ND & $\mathbf{0}$ & $\mathbf{0}$ & $\mathbf{0}$ & & & & & & & & & \\
\hline \multicolumn{15}{|c|}{ Wells that monitor the uppermost confined aquifer } \\
\hline $399-1-9$ & $\mathrm{C}$ & ND & $\mathbf{0}$ & $\mathbf{0}$ & & & & & & & & & & \\
\hline $399-1-16 C$ & $\mathrm{C}$ & ND & & & $\mathbf{0}$ & & & & & & & & & \\
\hline 399-1-17C & $\mathrm{C}$ & ND & & & & $\mathbf{0}$ & & & & & & & & \\
\hline $399-1-18 C$ & $\mathrm{C}$ & 1 & & & & & & & & & & & & \\
\hline 399-8-5B & $\mathrm{C}$ & 1 & $\mathbf{0}$ & ND & $\mathbf{0}$ & & & & & & & & & \\
\hline $399-8-5 \mathrm{C}$ & $\mathrm{C}$ & 1 & $\mathrm{ND}$ & ND & ND & & & & & & & & & \\
\hline 699-S29-E16C & $\mathrm{C}$ & ND & $\mathbf{0}$ & $\mathrm{ND}$ & $\mathbf{0}$ & & & & & & & & & \\
\hline \multicolumn{15}{|c|}{$\begin{array}{l}\text { (a) Well out-of-service. } \\
\text { Note: Maximum values for nitrate at } 300 \text { Area wells. Values in bold in shaded cells are below the EPA drinking water standard (45 mg/L). } \\
\text { Blanks indicate "no results." } \\
\text { BU = Bottom of unconfined aquifer. } \\
\text { C }=\text { First confined aquifer. } \\
\text { EPA }=\text { U.S. Environmental Protection Agency. } \\
\text { ND }=\text { Not detected. } \\
\text { TU }=\text { Top of unconfined aquifer. } \\
\text { TU-S }=\text { Top of unconfined aquifer at shoreline. }\end{array}$} \\
\hline
\end{tabular}




\subsection{8-11 Burial Ground Contaminants of Concern or Potential Concern}

Groundwater beneath the 618-11 burial ground, the northernmost outlying sub-region of the 300-FF-5 Operable Unit, contains contaminants associated with the site-wide plume, which originates in the 200 East Area (200-PO-1 Operable Unit). Release of tritium from materials in the burial ground has also affected groundwater, creating a plume of limited extent (Figure 2.10), but relatively high concentrations that exceed standards. The timing and mechanism causing the groundwater impact is not fully known. The principal burial ground release that has impacted groundwater is tritium, presumably as the result of out-gassing of tritium from irradiated lithium target material that was disposed to the burial ground. Several other constituents have exceeded the EPA drinking water standards in groundwater near the 61811 burial ground or are useful indicators of contamination and are, therefore, carried as COPC for the operable unit.

\subsubsection{Tritium}

Tritium concentrations at downgradient monitoring wells close to the 618-11 burial ground show a decreasing trend since the occurrence of peak values in 2000 (Table 2.11). The highest current concentration $(\sim 2.3$ million $\mathrm{pCi} / \mathrm{L})$ still greatly exceeds the drinking water standard. Relatively constant or gradually increasing trends are observed at wells along the downgradient flow path from the burial ground, suggesting dispersal of a "pulse" possibly created some time prior to 2000. Concentration trends for key plume tracking wells are shown in Figure 2.11. The general shape of the tritium plume has remained nearly constant since the first maps were drawn in 2000.

\subsubsection{Other Contaminants of Potential Concern}

Gross Beta - Recent results for gross beta at most plume monitoring wells are below the $50-\mathrm{pCi} / \mathrm{L}$ drinking water standard (Table 2.11). Elevated gross beta results have been observed at three sites close to the east perimeter fence of the burial ground: well 699-12-2C (maximum value of $98 \mathrm{pCi} / \mathrm{L}$ in 2002); well 699-13-3A (maximum value of 84 pCi/L in 2001); and borehole C3265 (maximum value of $271 \mathrm{pCi} / \mathrm{L}$ in 2000). These gross beta levels seem too high to be accounted for by tritium, which is a weak beta emitter. Technetium-99, which is associated with the site-wide plume, is the only other beta emitting radionuclide known to be present in the area (see description below).

Uranium - Uranium concentrations in monitoring wells at the 618-11 burial ground are all well below the $30-\mu \mathrm{g} / \mathrm{L}$ drinking water standard (Table 2.11), and probably reflect natural background levels in the sediment. There is no evidence indicating uranium releases from the burial ground.

Technetium-99 - The only other known beta emitter in the 618-11 burial ground area is technetium-99. The few results available (Table 2.12) suggest maximum concentrations in recent years of $\sim 320 \mathrm{pCi} / \mathrm{L}$, which could account for the gross beta values.

Technetium-99 migrates into the area via the site-wide plume but at low concentrations in recent years. For example, concentrations near the 316-4 cribs/618-10 burial ground sub-region, which is near the leading edge of the site-wide plume, currently fall in the range of 20 to $40 \mathrm{pCi} / \mathrm{L}$. The possibility exists that the few relatively results near $618-11$ burial ground (i.e., $\sim 320 \mathrm{pCi} / \mathrm{L}$; Table 2.12) represent small patches of higher concentrations that migrated into the area during earlier periods, perhaps during the 1970s and 1980s, when gross beta data indicate the possibility of a site-wide techneticium-99 plume 
in the range of 900 to $1,800 \mathrm{pCi} / \mathrm{L}$ at wells (699-26-15A and 699-20-20) located northwest of the burial ground. These higher concentrations persist in the area because of relatively low transmissivity sediment in the sub-region.

Gross Alpha - Gross alpha concentrations, which typically reflect uranium, fall below the 15-pCi/L drinking water standard in wells monitoring the 618-11 burial ground (Table 2.12), with one possible exception. A recent sample from well 699-13-3A, located adjacent to the east side fence of the burial ground, yielded a result of $15.4 \mathrm{pCi} / \mathrm{L}$ during 2004. This value is somewhat higher than the wellestablished historical trend in the 2 to $9 \mathrm{pCi} / \mathrm{L}$ range for the well, and may be an outlier.

Nitrate - Nitrate exceeds the 45-mg/L drinking water standard at several wells in the 618-11 burial ground monitoring network (Table 2.12). The source for the nitrate is unknown, but is likely to be related to the site-wide plume. Higher values persist longer in this sub-region for the same reasons as technetium-99, i.e., isolated pockets in relatively low-transmissivity sediment.

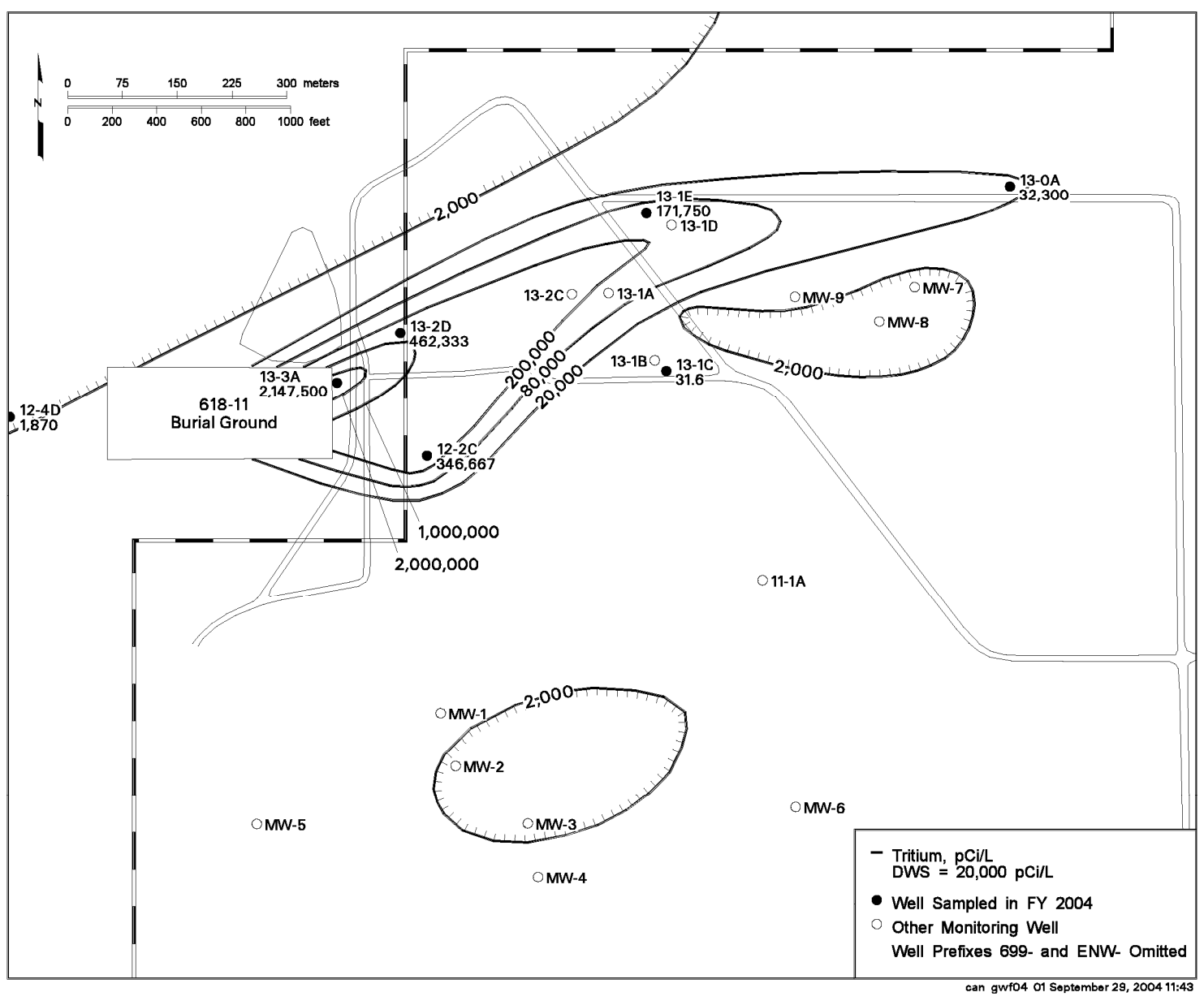

Figure 2.10. Tritium Plume Downgradient of 618-11 Burial Ground, 2004 Conditions 

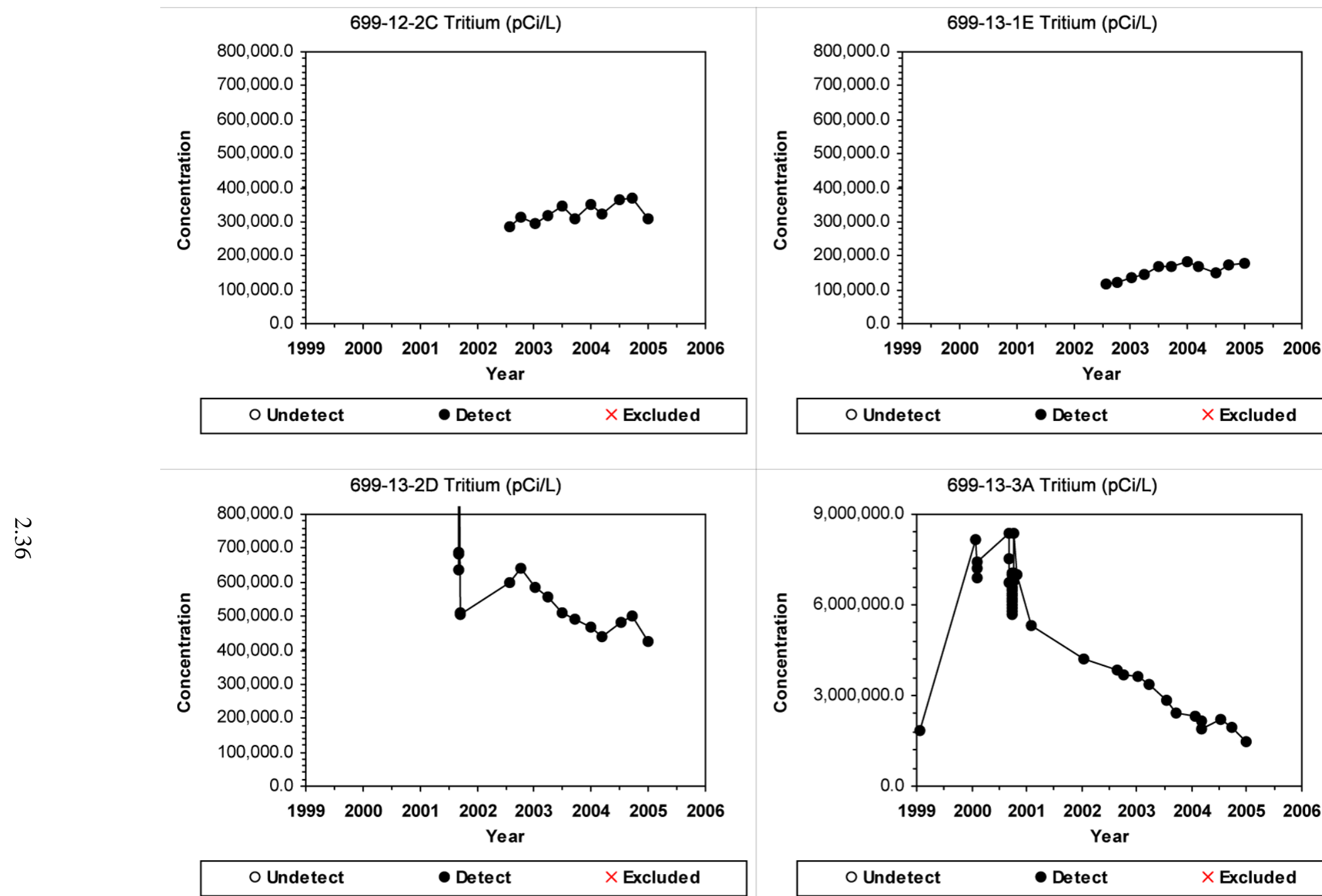

\begin{tabular}{|c|c|c|}
\hline O Undetect & - Detect & $\times$ Excluded \\
\hline
\end{tabular}

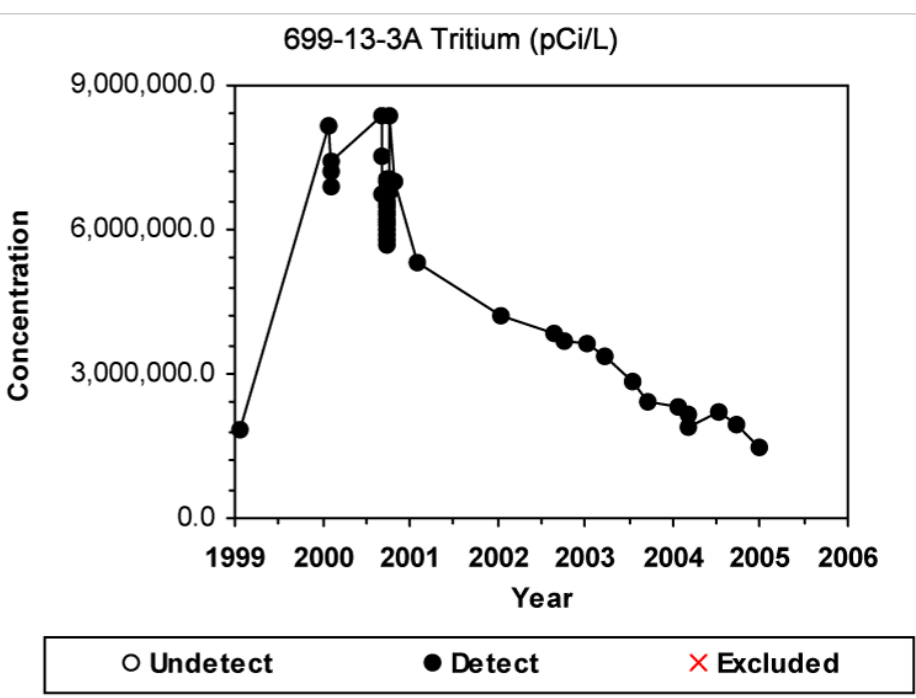

Figure 2.11. Tritium Concentrations at Wells Near the 618-11 Burial Ground 
Table 2.11. Maximum Concentrations for Contaminants of Potential Concern at 618-11 Sub-Region Wells: Group A

\begin{tabular}{|c|c|c|c|c|c|c|c|c|c|c|c|c|}
\hline Well Name & Unit & 1994 & 1995 & 1996 & 1997 & 1998 & 1999 & 2000 & 2001 & 2002 & 2003 & 2004 \\
\hline \multicolumn{13}{|c|}{ Tritium (pCi/L; MCL = 20,000) } \\
\hline $699-12-2 C$ & $\mathrm{TU}$ & & & & & & & & & 313,000 & 353,000 & 368,000 \\
\hline 699-12-4D & $\mathrm{TU}$ & & & & & & & 2,220 & 2,010 & & & 1,870 \\
\hline 699-13-0A & $\mathrm{TU}$ & & & & & & & & 21,600 & 35,500 & 36,400 & 35,000 \\
\hline 699-13-1A & $\mathrm{TU}$ & & & & & & & 23,300 & 16,400 & & & 139,000 \\
\hline 699-13-1B & $\mathrm{TU}$ & & & & & & & 32,400 & & & & \\
\hline 699-13-1C & $\mathrm{TU}$ & 770 & ND & & & & & ND & 25 & & 32 & \\
\hline 699-13-1D & $\mathrm{TU}$ & & & & & & & & 116,000 & & & \\
\hline 699-13-1E & $\mathrm{TU}$ & & & & & & & & & 123,000 & 184,000 & 179,000 \\
\hline 699-13-2C & $\mathrm{TU}$ & & & & & & & & 196,000 & & & \\
\hline 699-13-2D & $\mathrm{TU}$ & & & & & & & & $1,390,000$ & 639,000 & 587,000 & 502,000 \\
\hline 699-13-3A & $\mathrm{TU}$ & & & & & & $1,860,000$ & $8,380,000$ & $5,290,000$ & $4,230,000$ & $3,620,000$ & $2,320,000$ \\
\hline 699-14-E6S & $\mathrm{TU}$ & & ND & & & & & & & & & \\
\hline C3252 & $\mathrm{TU}$ & & & & & & & & 2,770 & & & \\
\hline C3255 & $\mathrm{TU}$ & & & & & & & & 1,040 & & & \\
\hline C3264 & $\mathrm{TU}$ & & & & & & & 6,510 & & & & \\
\hline C3265 & $\mathrm{TU}$ & & & & & & & $1,550,000$ & & & & \\
\hline ENW-MW-7 & $\mathrm{TU}$ & & & & & & & 509 & & & & \\
\hline ENW-MW-8 & $\mathrm{TU}$ & & & & & & & 351 & & & & \\
\hline ENW-MW-9 & $\mathrm{TU}$ & & & & & & & 4,580 & & & & \\
\hline ENW-MW-31 & $\mathrm{TU}$ & & & & & & & ND & & & & \\
\hline ENW-MW-32 & $\mathrm{TU}$ & & & & & & & ND & & & & \\
\hline \multicolumn{13}{|c|}{ Gross Beta $(\mathrm{pCi} / \mathrm{L} ; \mathrm{MCL}=50)$} \\
\hline 699-12-2C & $\mathrm{TU}$ & & & & & & & & & 98 & 86 & 81 \\
\hline 699-12-4D & $\mathrm{TU}$ & & 9 & 11 & & & & 9 & & & & \\
\hline 699-13-0A & $\mathrm{TU}$ & & & & & & & & 30 & 12 & 12 & 11 \\
\hline 699-13-1A & $\mathrm{TU}$ & & 33 & 21 & & & & 10 & 11 & & & 22 \\
\hline $699-13-1 \mathrm{~B}$ & $\mathrm{TU}$ & & 27 & 29 & & & & 18 & & & & \\
\hline $699-13-1 C$ & $\mathrm{TU}$ & & & & & & & 7 & 7 & & 4 & \\
\hline 699-13-1D & $\mathrm{TU}$ & & & & & & & & 20 & & & \\
\hline 699-13-1E & $\mathrm{TU}$ & & & & & & & & & 25 & 23 & 23 \\
\hline 699-13-2C & $\mathrm{TU}$ & & & & & & & & 33 & & & \\
\hline 699-13-2D & $\mathrm{TU}$ & & & & & & & & 25 & 41 & 37 & 31 \\
\hline 699-13-3A & $\mathrm{TU}$ & & 14 & 19 & 18 & 25 & 30 & 38 & 84 & 23 & 20 & 20 \\
\hline C3252 & $\mathrm{TU}$ & & & & & & & & 22 & & & \\
\hline C3255 & $\mathrm{TU}$ & & & & & & & & 26 & & & \\
\hline C3264 & $\mathrm{TU}$ & & & & & & & ND & & & & \\
\hline C3265 & $\mathrm{TU}$ & & & & & & & 271 & & & & \\
\hline ENW-MW-7 & $\mathrm{TU}$ & & & & & & & 6 & & & & \\
\hline ENW-MW-8 & $\mathrm{TU}$ & & & & & & & 8 & & & & \\
\hline ENW-MW-9 & $\mathrm{TU}$ & & & & & & & 22 & & & & \\
\hline ENW-MW-31 & $\mathrm{TU}$ & & & & & & & 7 & & & & \\
\hline ENW-MW-32 & $\mathrm{TU}$ & & & & & & & 8 & & & & \\
\hline
\end{tabular}


Table 2.11. (contd)

\begin{tabular}{|c|c|c|c|c|c|c|c|c|c|c|c|c|}
\hline Well Name & Unit & 1994 & 1995 & 1996 & 1997 & 1998 & 1999 & 2000 & 2001 & 2002 & 2003 & 2004 \\
\hline \multicolumn{13}{|c|}{ Uranium $(\mu \mathrm{g} / \mathrm{L} ; \mathrm{MCL}=30)$} \\
\hline $699-12-2 C$ & $\mathrm{TU}$ & & & & & & & & & 10 & 11 & 10 \\
\hline 699-12-4D & $\mathrm{TU}$ & & 6 & 5 & & & & 6 & & & & \\
\hline 699-13-0A & $\mathrm{TU}$ & & & & & & & & & 6 & 6 & 6 \\
\hline 699-13-1A & $\mathrm{TU}$ & & 6 & 5 & & & & 4 & & & & \\
\hline 699-13-1B & $\mathrm{TU}$ & & 8 & 10 & & & & 10 & & & & \\
\hline $699-13-1 \mathrm{E}$ & $\mathrm{TU}$ & & & & & & & & & 8 & 9 & 9 \\
\hline 699-13-2D & $\mathrm{TU}$ & & & & & & & & & 11 & 11 & 11 \\
\hline 699-13-3A & $\mathrm{TU}$ & & 8 & 10 & 9 & 9 & 12 & 11 & 11 & 10 & 10 & 10 \\
\hline ENW-MW-7 & $\mathrm{TU}$ & & & & & & & 2 & & & & \\
\hline ENW-MW-9 & $\mathrm{TU}$ & & & & & & & 31 & & & & \\
\hline \multicolumn{13}{|c|}{$\begin{array}{l}\text { Note: Maximum values for constituent at } 618-11 \text { burial ground wells. Values in bold in shaded cells are below the EPA drinking water standard } \\
\text { (value units). Blanks indicate "no results." } \\
\text { BU = Bottom of unconfined aquifer. } \\
\text { C }=\text { First confined aquifer. } \\
\text { EPA }=\text { U.S. Environmental Protection Agency. } \\
\text { MCL }=\text { Maximum contaminant level. } \\
\text { ND }=\text { Not detected. } \\
\text { TU }=\text { Top of unconfined aquifer. } \\
\text { TU-S }=\text { Top of unconfined aquifer at shoreline. }\end{array}$} \\
\hline
\end{tabular}


Table 2.12. Maximum Concentrations for Contaminants of Potential Concern at 618-11 Sub-Region Wells: Group B

\begin{tabular}{|c|c|c|c|c|c|c|c|c|c|c|c|c|c|c|}
\hline Well Name & Unit & 1992 & 1993 & 1994 & 1995 & 1996 & 1997 & 1998 & 1999 & 2000 & 2001 & 2002 & 2003 & 2004 \\
\hline \multicolumn{15}{|c|}{ Technetium-99 (pCi/L; MCL = 900) } \\
\hline $699-12-2 C$ & $\mathrm{TU}$ & & & & & & & & & & & & 319 & \\
\hline 699-12-4D & TU & & & & & & & & & ND & & & & \\
\hline 699-13-0A & $\mathrm{TU}$ & & & & & & & & & & ND & & & \\
\hline $699-13-1 \mathrm{~A}$ & TU & & & & & & & & & 30 & & & & \\
\hline 699-13-1B & $\mathrm{TU}$ & & & & & & & & & 28 & & & & \\
\hline $699-13-1 C$ & TU & & & & & & & & & ND & & & & \\
\hline 699-13-1D & $\mathrm{TU}$ & & & & & & & & & & ND & & & \\
\hline $699-13-2 C$ & $\mathrm{TU}$ & & & & & & & & & & 9 & & & \\
\hline 699-13-2D & $\mathrm{TU}$ & & & & & & & & & & 10 & & & \\
\hline 699-13-3A & $\mathrm{TU}$ & & & & & & & & & 124 & & & 12 & 26 \\
\hline C3252 & $\mathrm{TU}$ & & & & & & & & & & ND & & & \\
\hline C3255 & TU & & & & & & & & & & ND & & & \\
\hline C3264 & TU & & & & & & & & & ND & & & & \\
\hline C3265 & $\mathrm{TU}$ & & & & & & & & & 332 & & & & \\
\hline ENW-MW-7 & $\mathrm{TU}$ & & & & & & & & & ND & & & & \\
\hline ENW-MW-8 & $\mathrm{TU}$ & & & & & & & & & ND & & & & \\
\hline ENW-MW-9 & $\mathrm{TU}$ & & & & & & & & & ND & & & & \\
\hline ENW-MW-31 & $\mathrm{TU}$ & & & & & & & & & ND & & & & \\
\hline ENW-MW-32 & TU & & & & & & & & & ND & & & & \\
\hline \multicolumn{15}{|c|}{ Gross Alpha (pCi/L; MCL = 15) } \\
\hline $699-12-2 \mathrm{C}$ & $\mathrm{TU}$ & & & & & & & & & . & & 6 & 8 & 9 \\
\hline 699-12-4D & $\mathrm{TU}$ & & & & 4 & 4 & & & & 3 & & & & \\
\hline 699-13-0A & $\mathrm{TU}$ & & & & & & & & & & ND & 7 & 6 & 4 \\
\hline $699-13-1 \mathrm{~A}$ & $\mathrm{TU}$ & & & & 5 & 4 & & & & 4 & 3 & & & 4 \\
\hline $699-13-1 B$ & $\mathrm{TU}$ & & & & 6 & 5 & & & & 6 & & & & \\
\hline 699-13-1C & $\mathrm{TU}$ & & & & & & & & & ND & $\mathrm{ND}$ & & ND & \\
\hline 699-13-1D & $\mathrm{TU}$ & & & & & & & & & & ND & & & \\
\hline $699-13-1 \mathrm{E}$ & $\mathrm{TU}$ & & & & & & & & & & & 6 & 5 & 6 \\
\hline $699-13-2 \mathrm{C}$ & $\mathrm{TU}$ & & & & & & & & & & $\mathrm{ND}$ & & & \\
\hline 699-13-2D & $\mathrm{TU}$ & & & & & & & & & & 4 & 7 & 8 & 6 \\
\hline 699-13-3A & $\mathrm{TU}$ & & & & 5 & 8 & 5 & 8 & 5 & 9 & 2 & 5 & 8 & 9 \\
\hline C3252 & $\mathrm{TU}$ & & & & & & & & & & 10 & & & \\
\hline C3255 & $\mathrm{TU}$ & & & & & & & & & & $\mathrm{ND}$ & & & \\
\hline C3264 & $\mathrm{TU}$ & & & & & & & & & ND & & & & \\
\hline C3265 & $\mathrm{TU}$ & & & & & & & & & ND & & & & \\
\hline ENW-MW-7 & $\mathrm{TU}$ & & & & & & & & & ND & & & & \\
\hline ENW-MW-8 & $\mathrm{TU}$ & & & & & & & & & 6 & & & & \\
\hline ENW-MW-9 & $\mathrm{TU}$ & & & & & & & & & 23 & & & & \\
\hline ENW-MW-31 & $\mathrm{TU}$ & & & & & & & & & ND & & & & \\
\hline ENW-MW-32 & $\mathrm{TU}$ & & & & & & & & & 2 & & & & \\
\hline
\end{tabular}


Table 2.12. (contd)

\begin{tabular}{|c|c|c|c|c|c|c|c|c|c|c|c|c|c|c|}
\hline "Well Name & Unit & 1992 & 1993 & 1994 & 1995 & 1996 & 1997 & 1998 & 1999 & 2000 & 2001 & 2002 & 2003 & 2004 \\
\hline \multicolumn{15}{|c|}{ Nitrate $(\mu \mathrm{g} / \mathrm{L}$; MCL $=45)$} \\
\hline 699-12-2C & TU & & & & & & & & & & & 88 & 134 & 111 \\
\hline 699-12-4D & $\mathrm{TU}$ & & & & 26 & 25 & & & & 28 & 30 & & & 28 \\
\hline 699-13-0A & TU & & & & & & & & & & & 17 & 20 & 18 \\
\hline 699-13-1A & TU & & & & 48 & 24 & & & & 7 & 5 & & & 14 \\
\hline 699-13-1B & TU & & & & 43 & 61 & & & & 41 & & & & \\
\hline 699-13-1C & TU & 1 & & 0 & ND & & & & & $\mathbf{0}$ & $\mathrm{ND}$ & & $\mathbf{0}$ & \\
\hline 699-13-1E & $\mathrm{TU}$ & & & & & & & & & & & 43 & 53 & 60 \\
\hline 699-13-2D & $\mathrm{TU}$ & & & & & & & & & & & 54 & 66 & 64 \\
\hline 699-13-3A & TU & & & & 36 & 39 & & & & 103 & & 78 & 100 & 101 \\
\hline C3264 & $\mathrm{TU}$ & & & & & & & & & 35 & & & & \\
\hline C3265 & $\mathrm{TU}$ & & & & & & & & & 48 & & & & \\
\hline ENW-MW-7 & TU & & & & & & & & & 4 & & & & \\
\hline ENW-MW-8 & $\mathrm{TU}$ & & & & & & & & & 2 & & & & \\
\hline ENW-MW-9 & $\mathrm{TU}$ & & & & & & & & & 149 & & & & \\
\hline ENW-MW-31 & TU & & & & & & & & & $\mathbf{0}$ & & & & \\
\hline ENW-MW-32 & $\mathrm{TU}$ & & & & & & & & & $\mathbf{0}$ & & & & \\
\hline \multicolumn{15}{|c|}{$\begin{array}{l}\text { Note: Maximum values for constituent at 618-11 burial ground wells. Values in bold in shaded cells are below the EPA drinking water standard } \\
\text { (value units). Blanks indicate "no results." } \\
\text { BU = Bottom of unconfined aquifer. } \\
\mathrm{C}=\text { First confined aquifer. } \\
\text { EPA }=\text { U.S. Environmental Protection Agency. } \\
\text { MCL }=\text { Maximum contaminant level. } \\
\text { ND }=\text { Not detected. } \\
\text { TU }=\text { Top of unconfined aquifer. } \\
\text { TU-S }=\text { Top of unconfined aquifer at shoreline. }\end{array}$} \\
\hline
\end{tabular}




\subsection{6-4 Cribs/618-10 Burial Ground Contaminants of Potential Concern}

The second outlying sub-region contains the 316-4 cribs and 618-10 burial ground, where the COPC are uranium and tributyl phosphate. Groundwater was affected near these waste sites primarily during the early operating years, i.e., 1950s and 1960s, and are believed to be mostly associated with discharges to the 316-4 cribs. Solvents containing uranium were discharged to the open-bottom cribs, thus

contaminating the vadose zone and the underlying groundwater. Refurbishment of a monitoring well near the cribs in 1995 apparently remobilized some of the vadose zone contamination and caused increases in uranium and volatile organic compounds to appear in groundwater at that time.

Monitoring to date has not revealed evidence that groundwater beneath the 618-10 burial ground has been affected by releases from buried materials, as has occurred at the 618-11 burial ground to the north. A soil gas survey was conducted in September 2002 along the downgradient perimeter fence to determine if evidence for tritium was present and to help with positioning two new monitoring wells, which would augment the existing well network (Williams et al. 2003). High concentrations of helium-3, which would indicate tritium, were not found. Constituents associated with the leading edge of the site-wide plume that originated in the 200 East Area, including relatively low levels of tritium, are present in the vicinity of the 316-4/618-10 waste sites.

\subsubsection{Uranium}

Most monitoring results are below the $30-\mu \mathrm{g} / \mathrm{L}$ drinking water standard, but still above levels assumed to represent natural background conditions, i.e., 5 to $8 \mu \mathrm{g} / \mathrm{L}$ (Table 2.13). An exception is one recent result from new well 699-S6-E4L. This well is located along the downgradient side of the 618-10 burial ground and near the 316-4 crib, where remedial actions began during October 2004. The gradually increasing trend at this well (Figure 2.12) started prior to excavation activities, so remobilization associated with such activities as excavating of application of dust control water seems unlikely.

More frequent monitoring at wells 699-S6-E4L and 699-S6-E4A is being conducted as remediation of the 316-4 cribs proceeds (excavation remains open in March 2005). Soil samples collected during the excavation of the 316-4 cribs in October 2004, and again in December 2004 after the excavation was extended, revealed relatively high concentrations of uranium.

\subsubsection{Tributyl Phosphate}

This volatile organic compound was detected in groundwater during refurbishment of well 699-S6-E4A in 1995 at relatively high concentrations (reference), which have since decreased significantly (Table 2.13). The occurrence is localized at the well. Soil samples collected during the excavation of the 316-4 cribs in October 2004 revealed relatively high concentrations of tributyl phosphate. However, the compound is not very mobile in the environment, and is not expected to pose a threat of impacting groundwater. 


\subsubsection{Other Contaminants of Potential Concern}

Gross Alpha - All recent monitoring results are below the 15-pCi/L drinking water standard, although some alpha activity reflects the uranium in groundwater from past disposal to the 316-4 cribs (Table 2.14), in addition to natural uranium in the sediment.

Gross Beta - All recent monitoring results are below the $50-\mathrm{pCi} / \mathrm{L}$ drinking water standard (Table 2.14). Where gross beta is higher than natural background, the cause is attributed to technetium-99 brought to the area via the site-wide plume.

Nitrate, Technetium-99, and Tritium - These site-wide plume constituents are all currently present at concentrations below the drinking water standards (Table 2.15). Their presence reflects the site-wide plume from the 200 East Area (200-PO-1 Operable Unit), and each generally reveals a constant or declining concentration trend. 
Table 2.13. Maximum Concentrations for Contaminants of Potential Concern at 316-4/618-10 SubRegion Wells: Group A

\begin{tabular}{|c|c|c|c|c|c|c|c|c|c|c|c|c|c|c|}
\hline Well Name & Unit & 1992 & 1993 & 1994 & 1995 & 1996 & 1997 & 1998 & 1999 & 2000 & 2001 & 2002 & 2003 & 2004 \\
\hline \multicolumn{15}{|c|}{ Uranium $(\mu \mathrm{g} / \mathrm{L} ; \mathrm{MCL}=30)$} \\
\hline 699-S6-E4A & TU & & & & ND & 108 & 225 & 91 & 101 & 47 & 23 & 16 & 20 & 21 \\
\hline 699-S6-E4B & TU & & & & 5 & & & & & & & 6 & 6 & 6 \\
\hline 699-S6-E4CS & $\mathrm{TU}$ & & & & & & & 1 & $\mathbf{0}$ & $\mathbf{0}$ & $\mathbf{0}$ & & & \\
\hline 699-S6-E4CT & TU & & & & & 1 & & 2 & 2 & 1 & 1 & & & \\
\hline 699-S6-E4D & $\mathrm{TU}$ & 4 & 5 & 4 & 4 & & & 5 & 5 & 4 & 4 & 4 & 5 & 4 \\
\hline 699-S6-E4E & TU & & & & & & & & & & & 16 & & 12 \\
\hline 699-S6-E4K & $\mathrm{TU}$ & & & & & & & & & & & & 7 & 6 \\
\hline 699-S6-E4L & TU & & & & & & & & & & & & 29 & 35 \\
\hline 699-S6-E14A & TU & & & & & 5 & & & & & & & & \\
\hline 699-S11-E12A & TU & & & & & ND & & & & & & & & \\
\hline 699-S11-E12AP & TU & & & & $\mathbf{0}$ & ND & & & & & & & & \\
\hline B2764 & TU & & & & & 10 & & & & & & & & \\
\hline \multicolumn{15}{|c|}{ Tributyl Phosphate ( $\mu \mathrm{g} / \mathrm{L}$; MCL not established) } \\
\hline 699-S6-E4A & $\mathrm{TU}$ & & & & & 1,500 & 700 & 540 & 720 & 200 & 54 & 22 & 24 & \\
\hline 699-E4E & TU & & & & & & & & & & & & & ND \\
\hline 699-S6-E4K & $\mathrm{TU}$ & & & & & & & & & & & & ND & $\mathrm{ND}$ \\
\hline 699-S6-E4L & TU & & & & & & & & & & & & ND & $\mathrm{ND}$ \\
\hline B2763 & $\mathrm{TU}$ & & & & & ND & & & & & & & & \\
\hline B2764 & $\mathrm{TU}$ & & & & & ND & & & & & & & & \\
\hline \multicolumn{15}{|c|}{$\begin{array}{l}\text { Note: Maximum values for constituent at 618-11 burial ground wells. Values in bold in shaded cells are below the EPA drinking water standard } \\
\text { (value units). Blanks indicate "no results." } \\
\text { BU = Bottom of unconfined aquifer. } \\
\text { C }=\text { First confined aquifer. } \\
\text { EPA }=\text { U.S. Environmental Protection Agency. } \\
\text { MCL }=\text { Maximum contaminant level. } \\
\text { ND }=\text { Not detected. } \\
\text { TU }=\text { Top of unconfined aquifer. } \\
\text { TU-S }=\text { Top of unconfined aquifer at shoreline. }\end{array}$} \\
\hline
\end{tabular}



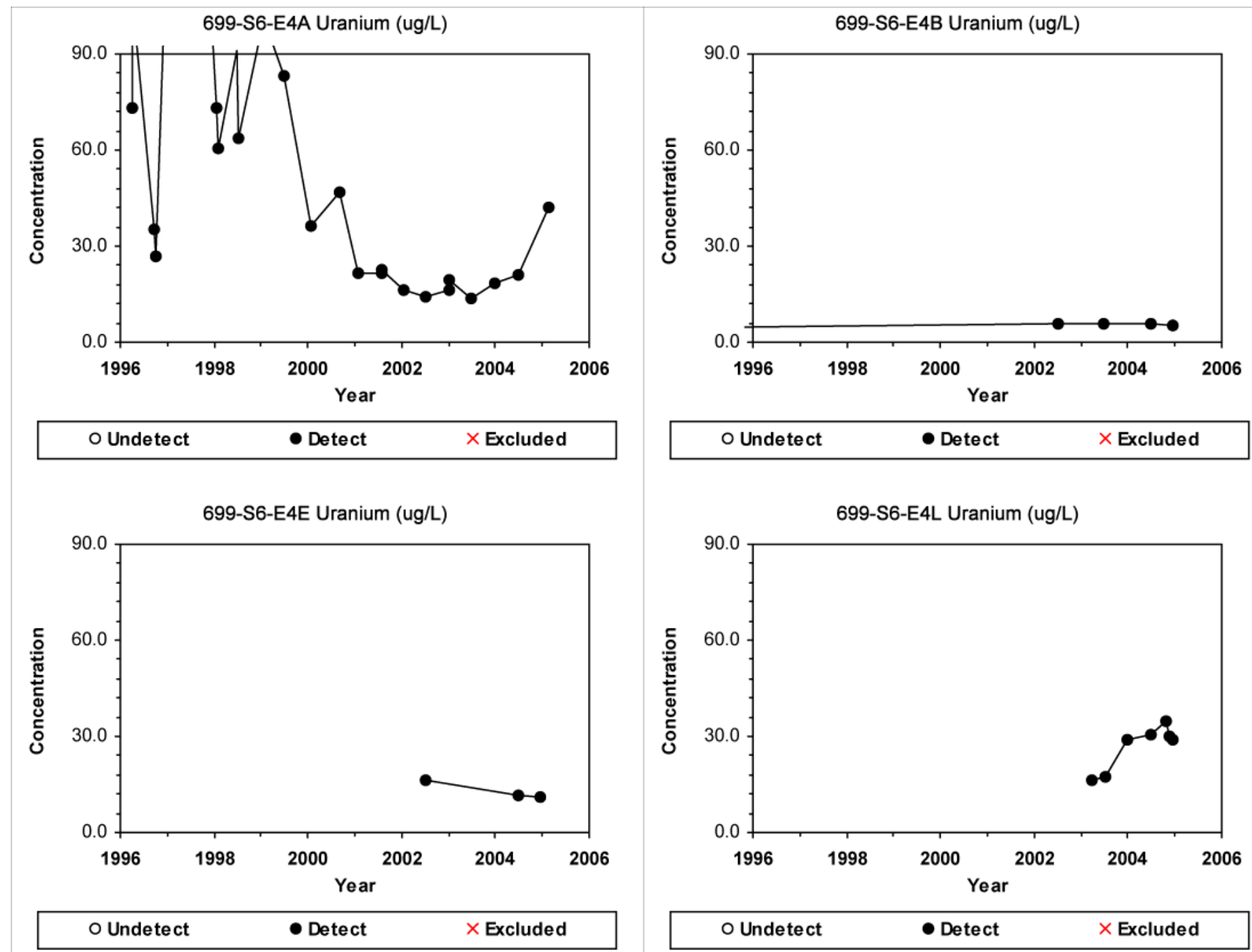

Figure 2.12. Uranium Concentrations at Wells Near the 316-4 Cribs Excavation Site 
Table 2.14. Maximum Observed Concentrations for Contaminants of Potential Concern at 316-4/618-10 Sub-Region Wells: Group B

\begin{tabular}{|c|c|c|c|c|c|c|c|c|c|c|c|c|c|c|}
\hline Well Name & Unit & 1992 & 1993 & 1994 & 1995 & 1996 & 1997 & 1998 & 1999 & 2000 & 2001 & 2002 & 2003 & 2004 \\
\hline \multicolumn{15}{|c|}{ Gross Alpha (pCi/L; MCL = 15) } \\
\hline 699-S3-E12 & $\mathrm{TU}$ & & & & & & & 2 & & ND & 3 & & $\mathrm{ND}$ & ND \\
\hline 699-S6-E4A & TU & & & & 319 & 15 & 112 & 42 & 45 & 23 & 10 & 8 & 14 & 15 \\
\hline 699-S6-E4B & TU & & & & 3 & & & & & & 6 & 3 & 3 & 4 \\
\hline 699-S6-E4D & $\mathrm{TU}$ & 5 & 5 & 4 & 4 & & 2 & 3 & ND & 4 & 4 & 3 & 4 & 3 \\
\hline 699-S6-E4E & $\mathrm{TU}$ & & & & & & & & & & & 10 & & 11 \\
\hline 699-S6-E4K & $\mathrm{TU}$ & & & & & & & & & & & & 4 & 6 \\
\hline 699-S6-E4L & $\mathrm{TU}$ & & & & & & & & & & & & 25 & 24 \\
\hline 699-S6-E14A & $\mathrm{TU}$ & & & & & 4 & 2 & 3 & & & 4 & & & 5 \\
\hline 699-S11-E12A & $\mathrm{TU}$ & & & & & 3 & 4 & 6 & & & & & & \\
\hline 699-S11-E12AP & $\mathrm{TU}$ & & & & & & ND & ND & & ND & & & & \\
\hline B2763 & $\mathrm{TU}$ & & & & & 7 & & & & & & & & \\
\hline B2764 & $\mathrm{TU}$ & & & & & 7 & & & & & & & & \\
\hline \multicolumn{15}{|c|}{ Gross Beta $(\mathrm{pCi} / \mathrm{L} ; \mathrm{MCL}=\mathbf{5 0})$} \\
\hline 699-S3-E12 & $\mathrm{TU}$ & & & & & & & 14 & & 12 & 16 & & 12 & 10 \\
\hline 699-S6-E4A & $\mathrm{TU}$ & & & & 258 & 31 & 50 & 74 & 47 & 29 & 24 & 20 & 18 & 22 \\
\hline 699-S6-E4B & $\mathrm{TU}$ & & & & 16 & & & & & & 19 & 16 & 16 & 18 \\
\hline 699-S6-E4D & TU & 21 & 18 & 16 & 16 & & 22 & 18 & 16 & 17 & 16 & 14 & 15 & 15 \\
\hline 699-S6-E4E & $\mathrm{TU}$ & & & & & & & & & & & 19 & & 17 \\
\hline 699-S6-E4K & $\mathrm{TU}$ & & & & & & & & & & & & 17 & 16 \\
\hline 699-S6-E4L & $\mathrm{TU}$ & & & & & & & & & & & & 22 & 26 \\
\hline 699-S6-E14A & $\mathrm{TU}$ & & & & & 7 & 8 & 9 & & & 9 & & & 9 \\
\hline 699-S11-E12A & $\mathrm{TU}$ & & & & & 10 & 13 & 12 & & & & & & \\
\hline 699-S11-E12AP & $\mathrm{TU}$ & & & & & & 8 & 8 & & 6 & & & & \\
\hline B2763 & $\mathrm{TU}$ & & & & & 30 & & & & & & & & \\
\hline B2764 & $\mathrm{TU}$ & & & & & 18 & & & & & & & & \\
\hline \multicolumn{15}{|c|}{$\begin{array}{l}\text { Note: Maximum values for constituent at 316-4/618-10 waste sites wells. Values in bold in shaded cells are below the EPA drinking water } \\
\text { standard (value units). Blanks indicate "no results." } \\
\text { BU = Bottom of unconfined aquifer. } \\
\text { C }=\text { First confined aquifer. } \\
\text { EPA }=\text { U.S. Environmental Protection Agency. } \\
\text { MCL }=\text { Maximum contaminant level. } \\
\text { ND }=\text { Not detected. } \\
\text { TU }=\text { Top of unconfined aquifer. } \\
\text { TU-S }=\text { Top of unconfined aquifer at shoreline. }\end{array}$} \\
\hline
\end{tabular}


Table 2.15. Maximum Concentrations for Site-Wide Plume Constituents at 316-4/618-10 Sub-Region Wells: Group C

\begin{tabular}{|c|c|c|c|c|c|c|c|c|c|c|c|c|c|c|}
\hline Well Name & Unit & 1992 & 1993 & 1994 & 1995 & 1996 & 1997 & 1998 & 1999 & 2000 & 2001 & 2002 & 2003 & 2004 \\
\hline \multicolumn{15}{|c|}{ "Nitrate $(\mathrm{mg} / \mathrm{L} ; \mathrm{MCL}=45)$} \\
\hline 699-S3-E12 & $\mathrm{TU}$ & 24 & 22 & 24 & 21 & & & 29 & & 21 & 27 & & 10 & 8 \\
\hline 699-S6-E4A & $\mathrm{TU}$ & & & & 5 & & & 29 & 25 & 27 & 27 & 27 & 28 & 29 \\
\hline 699-S6-E4B & $\mathrm{TU}$ & 19 & 18 & 20 & 20 & 18 & & & & & 27 & & & 27 \\
\hline 699-S6-E4CS & TU & & & & & 0 & & & & & & & & \\
\hline 699-S6-E4CT & $\mathrm{TU}$ & & & & & $\mathbf{0}$ & & & & & & & & \\
\hline 699-S6-E4D & $\mathrm{TU}$ & 26 & 25 & 26 & 24 & 24 & & & & & & & & \\
\hline 699-S6-E4E & $\mathrm{TU}$ & & & & & & & & & & & & & 24 \\
\hline 699-S6-E4K & $\mathrm{TU}$ & & & & & & & & & & & & 28 & 29 \\
\hline 699-S6-E4L & $\mathrm{TU}$ & & & & & & & & & & & & 39 & 49 \\
\hline 699-S6-E14A & $\mathrm{TU}$ & 5 & 6 & 7 & 6 & 6 & & & & & 6 & & & 6 \\
\hline 699-S11-E12A & $\mathrm{TU}$ & 22 & & 22 & 21 & & & 25 & & & & & & \\
\hline 699-S11-E12AP & $\mathrm{TU}$ & & ND & ND & $\mathbf{0}$ & $\mathbf{0}$ & & & & $\mathbf{0}$ & & & & ND \\
\hline B2763 & $\mathrm{TU}$ & & & & & 26 & & & & & & & & \\
\hline B2764 & $\mathrm{TU}$ & & & & & 29 & & & & & & & & \\
\hline \multicolumn{15}{|c|}{ Technetium-99 (pCi/L; MCL = 900) } \\
\hline 699-S3-E12 & $\mathrm{TU}$ & & & 17 & 19 & & & 36 & & 32 & 21 & & 21 & 18 \\
\hline 699-S6-E4A & $\mathrm{TU}$ & & & & & & & & & & & & 28 & \\
\hline 699-S6-E4B & $\mathrm{TU}$ & & & & & 25 & & & & & & & & \\
\hline 699-S6-E4D & $\mathrm{TU}$ & & & 34 & 34 & 32 & & & & & & & 20 & 22 \\
\hline 699-S6-E4K & $\mathrm{TU}$ & & & & & & & & & & & & 39 & 30 \\
\hline 699-S6-E4L & $\mathrm{TU}$ & & & & & & & & & & & & 33 & 36 \\
\hline 699-S6-E14A & $\mathrm{TU}$ & & & & & $\mathbf{0}$ & & & & & & & & \\
\hline 699-S11-E12A & $\mathrm{TU}$ & & & 11 & & 12 & & & & & & & & \\
\hline 699-S11-E12AP & $\mathrm{TU}$ & & & $\mathbf{0}$ & & & & & & & & & & \\
\hline B2763 & $\mathrm{TU}$ & & & & & 32 & & & & & & & & \\
\hline B2764 & $\mathrm{TU}$ & & & & & 24 & & & & & & & & \\
\hline \multicolumn{15}{|c|}{ Tritium (pCi/L; MCL = 20,000) } \\
\hline 699-S3-E12 & $\mathrm{TU}$ & 12,400 & 16,200 & 18,000 & 21,006 & 14,800 & 23,100 & 22,500 & & & & & 7,960 & 6,700 \\
\hline 699-S6-E4A & $\mathrm{TU}$ & & & & & & 28,700 & 26,700 & 24,000 & 21,300 & 20,200 & 19,400 & 17,100 & 15,500 \\
\hline 699-S6-E4B & $\mathrm{TU}$ & 27,500 & 28,600 & 29,300 & 26,400 & 24,387 & & & & & 19,700 & 17,100 & 17,000 & 16,800 \\
\hline 699-S6-E4CS & $\mathrm{TU}$ & & & & & 2,520 & 1,060 & ND & 421 & ND & ND & & & \\
\hline 699-S6-E4CT & $\mathrm{TU}$ & & & & & 27,800 & 6,660 & 1,500 & 691 & 387 & ND & & & \\
\hline 699-S6-E4D & $\mathrm{TU}$ & 39,300 & 38,900 & 37,300 & 36,000 & 31,741 & 31,100 & 28,200 & 23,600 & 20,400 & 19,800 & 17,900 & 17,800 & 13,500 \\
\hline 699-S6-E4E & $\mathrm{TU}$ & & & & & & & & & & & 17,100 & & 15,700 \\
\hline 699-S6-E4K & $\mathrm{TU}$ & & & & & & & & & & & & 14,500 & 14,700 \\
\hline 699-S6-E4L & $\mathrm{TU}$ & & & & & & & & & & & & 15,600 & 14,700 \\
\hline 699-S6-E14A & $\mathrm{TU}$ & 112 & 112 & 415 & $\mathrm{ND}$ & 150 & $\mathrm{ND}$ & $\mathrm{ND}$ & & & ND & & & ND \\
\hline 699-S11-E12A & $\mathrm{TU}$ & 8,330 & & 11,400 & 14,200 & 12,874 & 12,000 & 13,500 & & & & & & \\
\hline 699-S11-E12AP & $\mathrm{TU}$ & & 15 & $\mathbf{0}$ & ND & ND & ND & 6 & & 8 & & 19 & & ND \\
\hline \multicolumn{15}{|c|}{$\begin{array}{l}\text { Note: Maximum values for constituent at 316-4/618-10 waste sites wells. Values in bold in shaded cells are below the EPA drinking water } \\
\text { standard (value units). Blanks indicate "no results." } \\
\text { BU = Bottom of unconfined aquifer. } \\
\text { C }=\text { First confined aquifer. } \\
\text { EPA }=\text { U.S. Environmental Protection Agency. } \\
\text { MCL = Maximum contaminant level. } \\
\text { ND = Not detected. } \\
\text { TU = Top of unconfined aquifer. } \\
\text { TU-S }=\text { Top of unconfined aquifer at shoreline. }\end{array}$} \\
\hline
\end{tabular}




\subsection{Proposed Updates to Lists for Contaminants of Concern or Potential Concern}

COC or COPC have been identified in several regulatory documents, including the ROD (EPA 1996), explanation of significant difference to the ROD (EPA 2000), and first 5-year review (EPA 2001) for the 300-FF-5 Operable Unit. Abundant monitoring data have been collected during the period of interim remedial action for these groundwater constituents, which were determined to be of concern during the remedial investigation for the 300 Area sub-region of the operable unit (DOE 1995), and by other investigations at the outlying sub-regions (e.g., Dresel et al. 2000).

The data trends covering approximately the past 12 years (i.e., since the initial remedial investigation) for these constituents have been evaluated with respect to remedial action objectives for the operable unit. $^{3}$ Because of changes in trends and concentrations, an updated classification of these constituents is proposed. The re-classification scheme considers three categories: (1) retain as a COC, (2) retain as a COPC, or (3) remove from previous list of COC or COPC.

Retaining a constituent as a COC indicates that concentrations exceed standards, and implies continued monitoring and characterization of plume behavior. Re-evaluation of the remedy is warranted because of uncertainty in predicted plume behavior, and/or the potential for human health or ecological risk from the plume.

Retaining a constituent as a COPC indicates that the level of contamination remains above standards, but that there is evidence for decreasing trends, which are likely to continue, or that concentrations are approaching the relevant standards and are likely to continue to decrease. If there is limited information on the risk posed to human health or the environment for the constituent, it will be retained as a COPC while observed at concentrations above standards.

Removing a constituent from the list of COC or COPC is proposed for those constituents that are consistently observed at concentrations below the relevant regulatory standards. "Consistency" implies monitoring data for a period of 5 years, i.e., the length of a 5-year ROD review cycle. A second reason for removing a constituent from the list of 300-FF-5 contaminants is that the constituent is part of the sitewide plume (i.e., 200-PO-1 Operable Unit), or plumes that approach the 300 Area from the southwest (1100-EM-1 Operable Unit), both of which co-mingle with 300-FF-5 Operable Unit groundwater in some areas. Those constituents will continue to be monitored but will not be retained on the list of COC or COPC for the 300-FF-5 Operable Unit.

\subsubsection{Area}

The current list of contaminants for the 300 Area sub-region includes COC uranium, cis-1,2dichloroethene, and trichloroethene; and COPC tetrachloroethene, strontium-90, tritium, and nitrate.

\subsubsection{Proposed Contaminants List for the 300 Area}

The following contaminants will be retained:

\footnotetext{
${ }^{3}$ Remedial action objectives are listed in EPA (1996) and summarized in Chapter 1 of this report.
} 
- COC: Uranium

- COPC: cis-1,2-dichloroethene

The following contaminants will no longer be included:

- COC:

- cis-1,2-dichloroethene (below standards for all wells except one; detected at one shoreline site at concentration well below standard)

- Trichloroethene (generally below standards for last 5 years, but some unexplained occurrences near shoreline at levels near standards)

- COPC:

- $\quad$ Tetrachloroethene (below standard for last 5 years)

- $\quad$ Strontium-90 (below standard for last 5 years; limited to one well)

- $\quad$ Tritium (below standard for last 5 years; assign to 200-PO-1 Operable Unit)

- $\quad$ Nitrate (generally below standard for last 5 years, except for plume migrating into 300 Area from southwest; assign to 1100-EM-1 Operable Unit)

\subsubsection{Discussion}

Uranium contamination in the 300 Area persists at concentrations above the remedial action goal, i.e., the EPA drinking water standard $(30 \mathrm{pCi} / \mathrm{L})$. While monitoring data shows that uranium concentrations have decreased over the last 10 years, re-evaluation of the interim remedy is necessary because the rate of decrease in uranium concentration is significantly different than the rate of decrease expected and used as a basis for the remedy selection in the ROD (EPA 1996). The focus of these re-evaluation efforts will be to better quantify the natural attenuation rate and factors that affect this rate and to assess natural attenuation and other more active remedial alternatives to select a preferred remediation alternative for the future.

Cis-1,2-dichloroethene is contaminating a very localized portion of the aquifer in the 300 Area at a concentration that is above the remedial action goal, i.e. the EPA drinking water standard $(70 \mu \mathrm{g} / \mathrm{L})$. While the cis-1,2-dichloroethene concentration is not decreasing, it is also not known to be affecting any receptors and may decrease in concentration in the future. A specific timeframe for the natural attenuation remedy of cis-1,2-dichloroethene was not defined in the ROD (EPA 1996). Because there is no known human health or environmental impact from this contaminant, and there appears to be no expansion of the extent of contamination, the natural attenuation remedy is performing as outlined in the ROD. Thus, while it is proposed that cis-1,2-dichloroethene be retained as a COPC, no re-evaluation of the interim remedy is needed. Cis-1,2-dichloroethene will continue to be monitored at the localized area surrounding the one well where it is present at concentrations above the drinking water standard.

\subsubsection{8-11 Burial Ground}

The current list of contaminants for the 618-11 burial ground includes COPC tritium, gross beta, technetium-99, gross alpha, uranium, and nitrate.

\subsubsection{Proposed Contaminants List for the 618-11 Burial Ground}

The following contaminants will be retained: 
- COC: None

- COPC: Tritium, gross beta, and nitrate

The following contaminants will no longer be included:

- COC: None

- COPC:

- $\quad$ Technetium-99 (below standard for last 5 years)

- $\quad$ Gross alpha (generally below standard for last 5 years; exceptions are suspected outliers)

- $\quad$ Uranium (below standard for last 5 years; detected at levels suggestive of natural background)

\subsubsection{Discussion}

Tritium is contaminating groundwater near the 618-11 burial ground at concentrations greatly exceeding the EPA drinking water standard $(20,000 \mathrm{pCi} / \mathrm{L})$. Although currently considered unlikely, tritium will be retained as a COPC because of the potential future need by Energy Northwest to use groundwater from this area. Data analysis and numerical modeling suggest that the tritium concentrations will decrease to below the drinking water standard within approximately 20 years, primarily because of radioactive decay (tritium has a half-life of 12.3 years) and dispersion. The plume is not expected to reach the river at concentrations that would be of concern at exposure locations associated with the groundwater/river interface

A specific timeframe for natural attenuation was not defined in the explanation of significant difference (EPA 2000), where tritium was identified as a COPC for the 300-FF-5 Operable Unit. However, current data and analysis indicate that the tritium plume will meet all of the remedial action objectives through natural attenuation. Thus, while it is recommended that tritium be retained as a COPC, no re-evaluation of the remedy for tritium is needed. Tritium will continue to be monitored in the groundwater near the 618-11 burial ground as part of this remedy.

There is some uncertainty as to the cause and origin for elevated gross beta values and nitrate. There is no evidence to link these constituents with releases from the 618-11 burial ground. However, both are associated with the site-wide plume that originated in the 200 East Area (200-PO-1 Operable Unit). The dilemma is that concentrations in the 618-11 sub-region seem too high for current conditions in the 
site-wide plume. The current explanation is that these anomalously high values represent former sitewide plume levels, and that they are preserved near 618-11 because of relatively low transmissivity of the aquifer in the area.

\subsubsection{6-4 Cribs and 618-10 Burial Ground}

The current list of contaminants for the 316-4/618-10 sub-region includes COPC uranium and tributyl phosphate.

\subsubsection{Proposed Contaminants List for the 316-4 Cribs and 618-10 Burial Ground}

The following contaminants will be retained:

- COC: None

- COPC: Uranium and tributyl phosphate

The following contaminants will no longer be included:

- COC: None

- COPC: None

\subsubsection{Discussion}

No changes are proposed to the list of COPC for this sub-region of the 300-FF-5 Operable Unit. Several near-term activities may provide reasons for changing the list, e.g., the excavation of the 316-4 cribs in October to December 2004, and the initiation of a limited field investigation (including qualitative risk assessment), which has not yet been completed for this sub-region. 


\subsection{Conceptual Model for Uranium at the 300 Area}

This Chapter describes certain aspects of the conceptual site model ${ }^{1}$ for the 300 Area portion of the 300-FF-5 Operable Unit, i.e., the characteristics of uranium contamination in the vadose zone and aquifer beneath the 300 Area. The emphasis is on (a) hydrogeologic framework that contains the uranium, and (b) processes that act to transport uranium through the environment or to control uranium's mobility. Some of the information presented in this Chapter provides updates to earlier investigations of the 300 Area (e.g., Lindberg and Bond 1979; Schalla et al. 1988) and the initial remedial investigation report for the 300-FF-5 Operable Unit (DOE 1995). Other information is the product of very recent investigations, such as research involving the geochemistry of uranium in the 300 Area environment (Zachara et al. 2005) and analyses of trends in the level of uranium contamination in groundwater.

Background on Uranium Problem at the $\mathbf{3 0 0}$ Area. Historical operations involving fuel fabrication and research activities at the 300 Area have caused contamination of engineered liquid waste disposal facilities, the underlying vadose zone, and the uppermost aquifer by uranium (Young et al. 1990; Young and Fruchter 1991; DeFord et al. 1994). The greatest impact to groundwater from disposal of waste containing uranium probably occurred during the 1950s and 1960s, when effluent was directed to the 307 trench, north and south process ponds, and during the 1970s and 1980s to the 300 Area process trenches (see Figure 1.2 for location map). Some uranium in the groundwater plume may also have been widely distributed in the past during periods of unusually high water-table conditions. As conditions returned to more normal levels, groundwater containing uranium was left behind in the less than fully saturated capillary fringe above the water table.

A groundwater plume has been present beneath the 300 Area since disposal operations started. Its persistence indicates some level of re-supply from the vadose zone via mechanisms not yet clearly defined, because the rate of groundwater movement appears to be sufficient to have moved the plume away from the area in the absence of a continuing source. Following excavation of the most contaminated waste disposal sites and adjacent soil during the period 1995 to 2004, some uranium remains in the underlying vadose zone. Uranium in groundwater is currently transported to the Columbia River under natural hydrologic flow conditions, where it discharges into the river system and is dispersed via additional transport pathways (e.g., the free-flowing stream, biota and food chain, and sediment). Uranium is also being removed from the aquifer via a water supply well that has provided water for aquarium operations at the 331 Building since 1982.

\footnotetext{
${ }^{1}$ The EPA guidance for conducting remedial investigations (EPA 1988, p. 2-7) indicates that a "conceptual site model should include known and suspected sources of contamination, types of contaminants and affected media, known and potential routes of migration, and known or potential human and environmental receptors.” The stated purpose in the guidance for developing and maintaining a conceptual site model is "...to evaluate potential risks to human health and the environment..." and to "...assist in the identification of potential remedial technologies."
} 
Uranium in the 300 Area environment represents an actual or potential risk via the following pathways:

- Radiation dose to humans at the ground surface

- Radiation dose and ingestion hazards to terrestrial biota

- Ingestion hazards to humans who use groundwater as drinking water

- Human and biotic exposure to uranium-contaminated water at the rivershore

- Uptake of uranium by aquatic organisms that use the interface as habitat

The risk associated with some of these pathways has been significantly reduced by source removal actions during the period 1995 to 2004. Human health risks associated with groundwater has been managed by institutional controls on groundwater use. Recent efforts have been undertaken to better define contaminant distribution in various media along the shoreline (e.g., Patton et al. 2003), and risk assessments are underway for the Columbia River Corridor, including the 300 Area segment (Weiss 2005).

Key Components of Conceptual Site Model for Uranium. Figure 3.1 is a generalized cross section showing a former liquid waste disposal facility, the uranium plume, and the Columbia River (adapted from Lindberg and Chou 2001, their Figure 5.1). For this report, five zones are identified as having significance for anticipating the distribution and mobility of uranium contamination:

- Zone 1 represents the waste site and adjacent soil that has been removed as part of source remedial actions. While initially a conduit for supplying uranium to the subsurface, no future impact to the groundwater will occur. Backfill and surface cover materials will influence the degree that natural precipitation or water from human activities (e.g., irrigation) will infiltrate.

- Zone 2 is the vadose zone between the deepest part of the source excavation and the capillary fringe associated with the groundwater table. Relatively high concentrations of uranium are likely to have migrated through this zone during operations. Limited sampling within and beneath excavated waste sites indicates that some amount of uranium remains sorbed to sediment in this zone.

- Zone 3 is a zone defined by the maximum elevation of the capillary fringe associated with the water table and the minimum water-table elevation. During periods of unusually high water-table elevations (because of high river-stage conditions), uranium-contaminated groundwater would move into the lower vadose zone. When the water table returned to normal, some uranium would have been left behind in pore fluid and retained on soil particles, thus remaining as a potential source for plume resupply if unusually high water table elevations return.

- Zone 4 is the uppermost hydrologic unit through which uranium migrates toward the river. During migration, dissolved uranium interacts with aquifer solids to sorb or desorb, depending on geochemical conditions.

- Zone 5 is a highly dynamic zone of interaction between groundwater and river water that infiltrates the banks and channel substrate to varying degrees, depending on river state. Geochemical conditions change rapidly within this zone because of the contrast in certain characteristics of groundwater and river water. Dilution of contaminants in groundwater typically occurs in this zone, prior to the ultimate discharge of groundwater into the river system. 


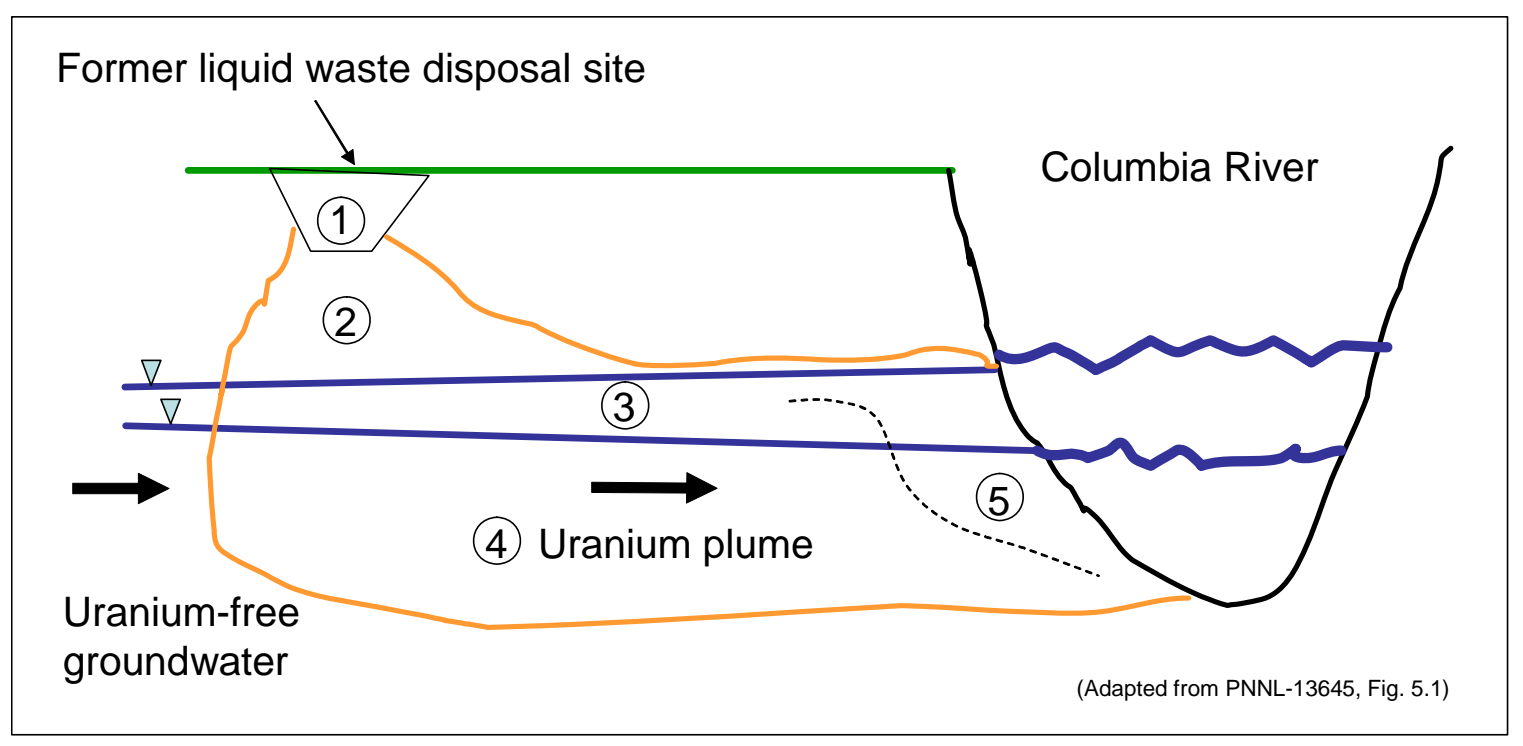

Figure 3.1. Diagram Illustrating Key Components of Conceptual Model for Uranium

Current Interim Remedial Action and Uranium. The heterogeneous stratigraphic characteristics and variable geochemical conditions within the area occupied by the uranium plume create challenges for computer simulations of plume behavior, which are used to predict future conditions and aid in designing technological solutions for lowering uranium concentrations. The features and processes in each of the zones described in Figure 3.1 influence the level of contamination and how it changes with time. Even though the basic elements of the uranium distribution and the plume's migration over time have been described in initial site models (e.g., DOE 1995), additional details on certain features and processes are needed to provide an appropriate technical basis for re-evaluating the current interim remedy. Key among these information needs is an improved estimate for the inventory of uranium in each zone.

In summary, the major issues associated with a conceptual site model for the uranium plume beneath the 300 Area involve (1) how much uranium from waste disposal operations remains in the environment; (2) how that amount varies with time and space; (3) if decreasing, when will the level reach criteria that are acceptable; and (4) is uranium causing an unacceptable impact to human health and biota. Currently, the phrase "acceptable criteria" means groundwater at concentrations lower than the EPA drinking water standard of $30 \mu \mathrm{g} / \mathrm{L}$. No water quality criteria for the protection of freshwater organisms have yet been listed for uranium, so the $30-\mu \mathrm{g} / \mathrm{L}$ value is used by default for groundwater at discharge locations associated with the Columbia River.

The following subsections contain descriptions for features and processes associated with uranium contamination in the 300 Area. The information provides the technical basis for moving forward with a re-evaluation of remedial action alternatives, and also keeps remedial action decision makers (e.g., DOE, EPA, and Washington State Department of Ecology [Ecology]) apprised of updated information regarding uranium contamination. These descriptions also establish a framework for numerical models that are used to estimate conditions away from points of observation and for predicting future conditions. The characteristics in each of the five zones shown in Figure 3.1 will determine the suitability of various technologies that could potentially be used to reduce uranium concentrations in groundwater. Finally, the combination of this conceptual site model for uranium, contaminant monitoring results, and exposure 
scenarios can be used to assess environmental impacts and to quantify risk associated with those impacts. These current and future activities are described in more detail in a work plan for the Phase III Focused Feasibility Study for the 300-FF-5 Operable Unit (DOE 2005).

\subsection{Hydrogeology of the 300 Area}

The hydrogeology of the 300 Area has been described in several previous reports (e.g., Lindberg and Bond 1979; Schalla et al. 1988; Gaylord and Poeter 1991; Swanson et al. 1992). Because no new boreholes have been completed since those reports were prepared, the observational data upon which to base a description remain essentially the same. However, new data management and visualization systems have improved the ability to use those data.

\subsubsection{Hydrogeologic Framework for the 300 Area}

Geologic descriptions are available from 67 boreholes in the 300 Area. All of these boreholes are deep enough to penetrate the contact between the Hanford and Ringold stratigraphic formations. ${ }^{2}$ Stratigraphic unit contacts inferred during three previous studies (Schalla et al. 1988; Swanson 1992; Cole et al. 2001) were reviewed and inconsistencies resolved when necessary. No new boreholes have been drilled since the previous studies were conducted. EarthVision geologic modeling and visualization software has been used to interpolate unit contacts between borehole locations and to create a threedimensional model of the hydrogeologic framework. Figure 3.2 provides an index to the three cross sections shown in Figures 3.3, 3.4, and 3.5, which are drawn through the model to illustrate the framework.

Highly transmissive Hanford formation gravels are found below the water table across portions of the 300 Area. The extent and thickness of saturated Hanford formation gravel vary as a consequence of changes in water-table elevation, which are caused by changes in the Columbia River stage. The saturated thickness of the Hanford formation in the 300 Area varies from 0 to 15 meters (0 to 49 feet). Aquifer pumping tests at five boreholes within the 300 Area reveal an average hydraulic conductivity of approximately 14,000 meters (45,932 feet) per day for saturated Hanford formation gravels. This indicates a highly transmissive hydrologic unit. The value is significantly higher than the average hydraulic conductivity for Hanford formation gravels elsewhere on the Hanford Site (i.e., approximately 2,000 meters (6,562 feet) per day.

Ringold Formation gravels below the water-table range in thickness from 15 to 50 meters (49 to 164 feet). Aquifer pumping tests at seven boreholes in the 300 Area suggest an average hydraulic conductivity of approximately 125 meters (410 feet) per day, which is again higher than the average values for Ringold gravels elsewhere on the Hanford Site but considerably less transmissive than the overlying Hanford unit. Relatively higher conductivities may exist in the upper part of the Ringold Formation (i.e., Unit E gravel), where most pumping tests have been performed.

\footnotetext{
${ }^{2}$ It is believed that most of the contaminated groundwater resides in the hydrologic unit formed by saturated Hanford sediment.
} 


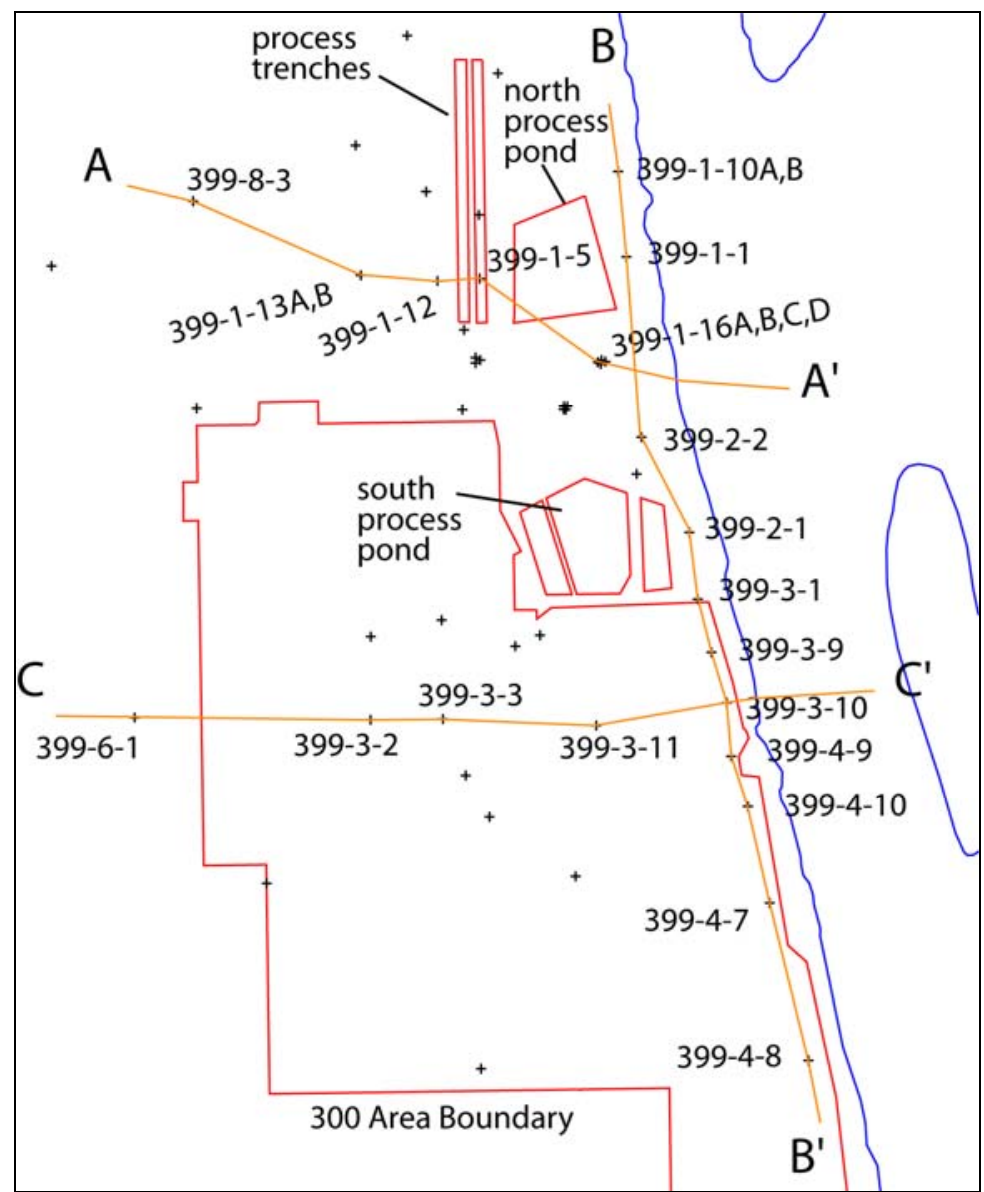

Figure 3.2. Index Map to Cross Sections of the 300 Area

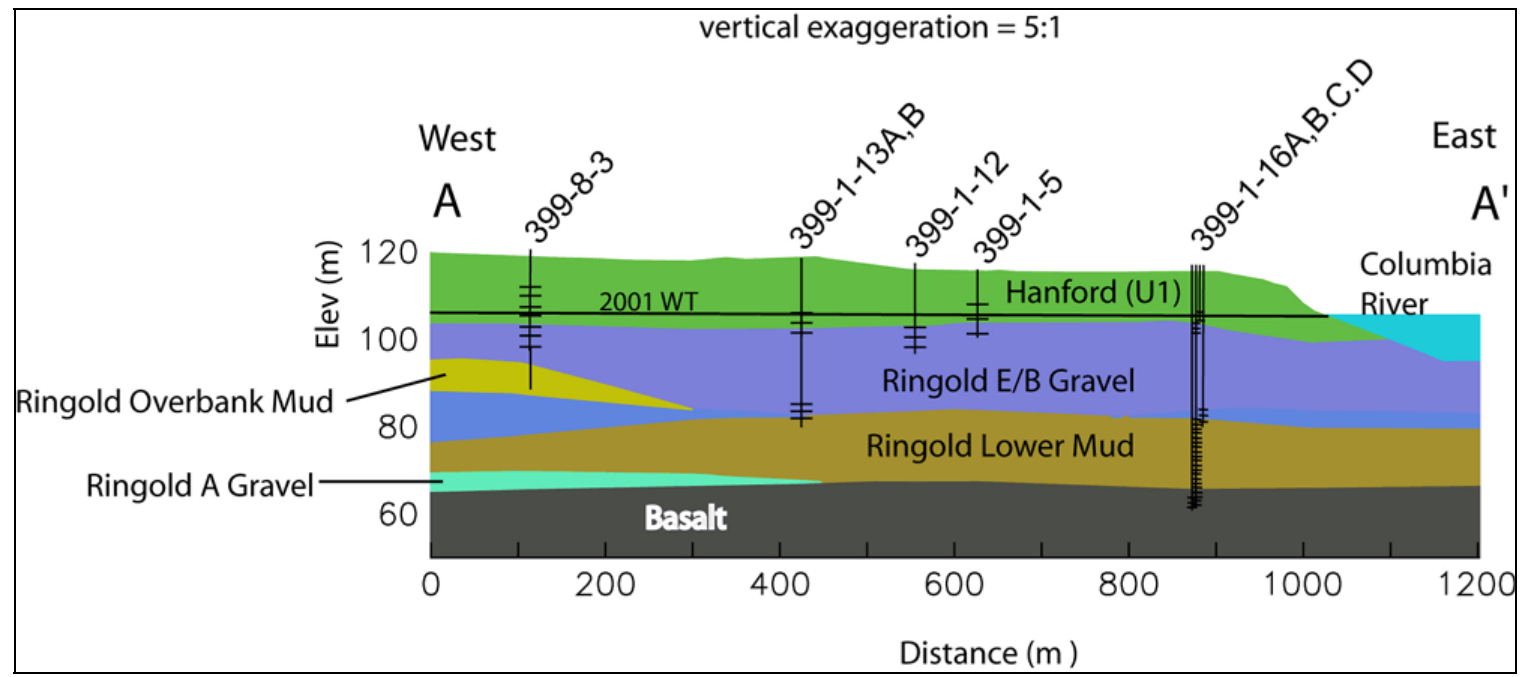

Figure 3.3. West-to-East Cross Section Along Flow Path from 300 Area Process Trenches to the Columbia River 


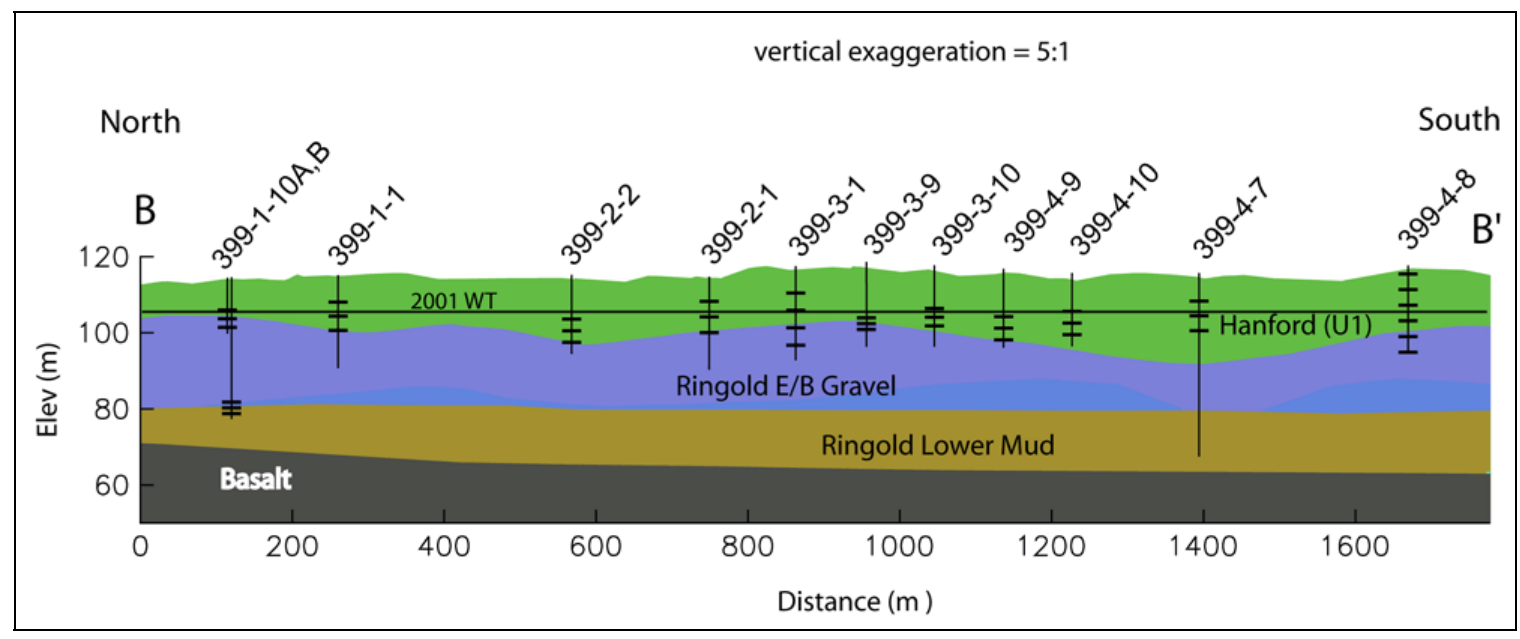

Figure 3.4. North-to-South Cross Section Along Shoreline Wells

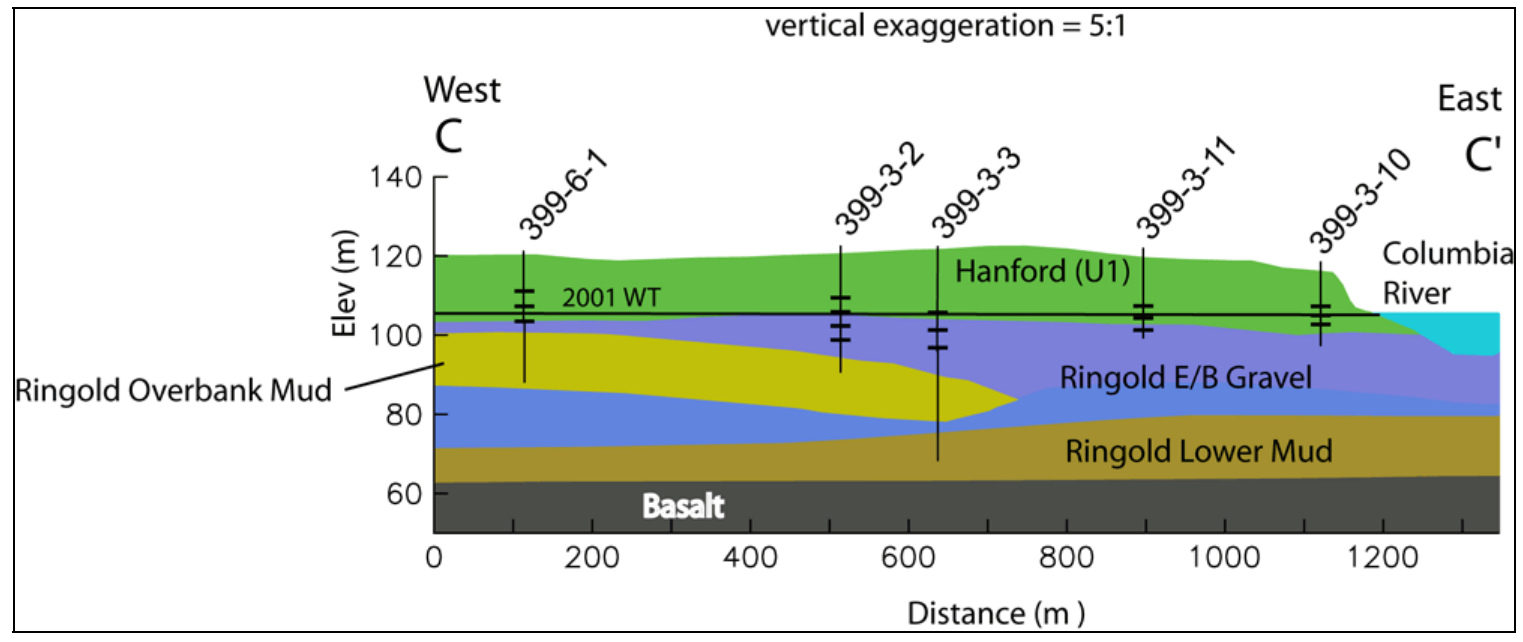

Figure 3.5. $\quad$ West-to-East Cross Section Through Central Portion of 300 Area

The EarthVision model establishes the hydrogeologic framework for three different simulation modeling tasks that are currently underway in 2005 for groundwater flow and transport beneath the 300 Area. Using the same hydrogeologic framework for each modeling task results in greater consistency in output from the various simulations.

\subsubsection{Groundwater Flow Conditions}

Groundwater flow beneath the 300 Area is generally directed toward the southeast. Groundwater appears to converge beneath the 300 Area, with flow coming into the 300 Area from the northwest, west, and southwest. The uppermost aquifer (Hanford formation) is highly transmissive because of open framework gravelly sediment, thus leading to high flow velocities (i.e., meters per day). However, because the hydraulic gradient that drives the flow varies with Columbia River stage, actual movement 
paths of water can be variable when viewed on short time scales, such as days or weeks. When viewed over seasons and years, the net flow and movement of contaminant plumes follows the generally southeasterly course.

Seasonal Variability in Water-Table Conditions. To better understand how this dynamic system might influence the dispersal pattern of contaminant plumes, hourly hydraulic head data were analyzed to (a) determine the predominant groundwater flow directions, and (b) assess variability in flow directions during the various seasons. The analysis used hourly measurements of hydraulic head made at 30 wells in the 300 Area during the period March 1992 through February 1993, using pressure transducers (Campbell and Newcomer 1992). Water-table elevation contour maps were prepared for March, May, June, September, and December 1992 (Figures 3.6, 3.7, 3.8, 3.9, and 3.10, respectively). The contours were based on 22 wells deemed most representative of unconfined aquifer (i.e., water table) conditions. The values contoured were averages of all hourly measurements made during a particular month.

The water-table maps for the various months reveal that the shape of the water table and, therefore, the inferred long-term groundwater flow pattern, appears to show little variation from season to season. ${ }^{3}$ The overall elevation of the water table is higher during the seasonal high river discharge that occurs in May and June. The aquifer apparently equilibrates rapidly to changes in river stage, which is expected given the high transmissibility of the stratigraphic units.

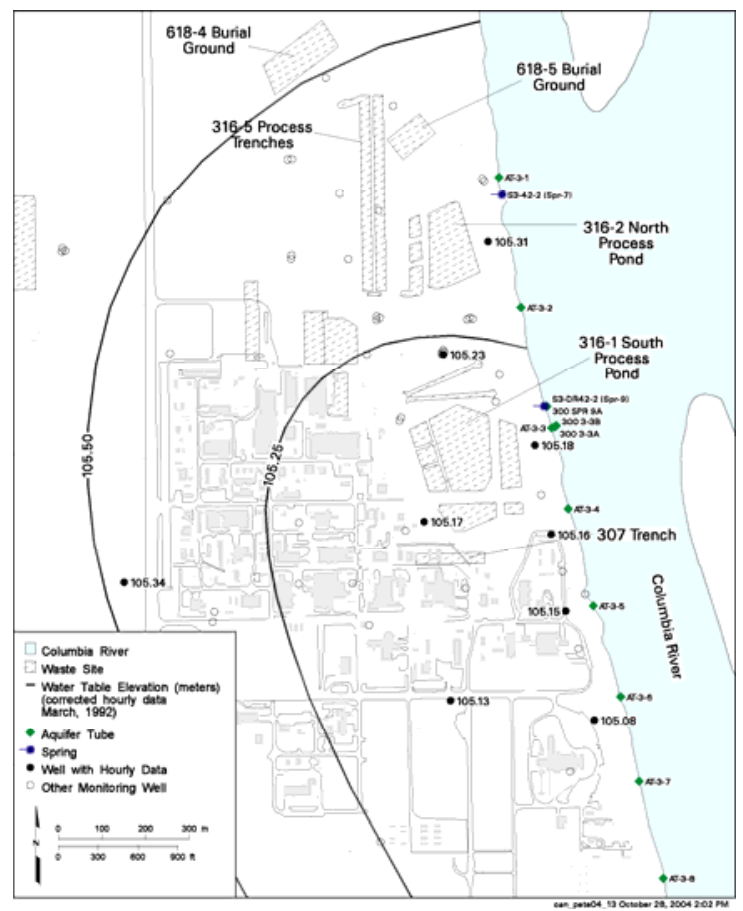

Figure 3.6. $\quad 300$ Area Water-Table Elevation, March 1992 (Averaged Hourly Data)

\footnotetext{
${ }^{3}$ Flow direction is generally perpendicular to elevation contours.
} 


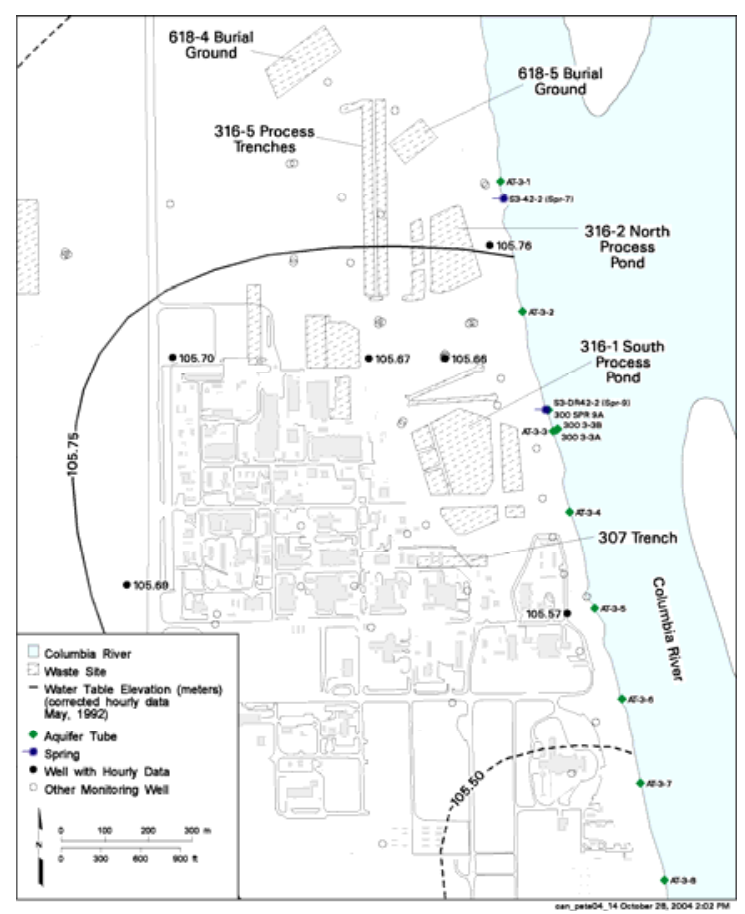

Figure 3.7. 300 Area Water-Table Elevation, May 1992 (Averaged Hourly Data)

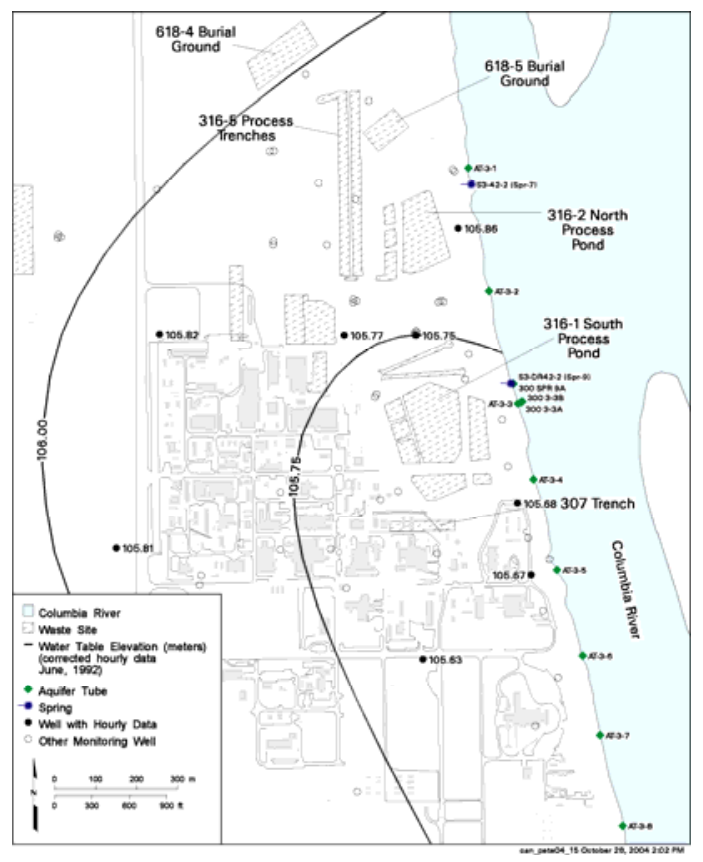

Figure 3.8. 300 Area Water-Table Elevation, June 1992 (Averaged Hourly Data) 


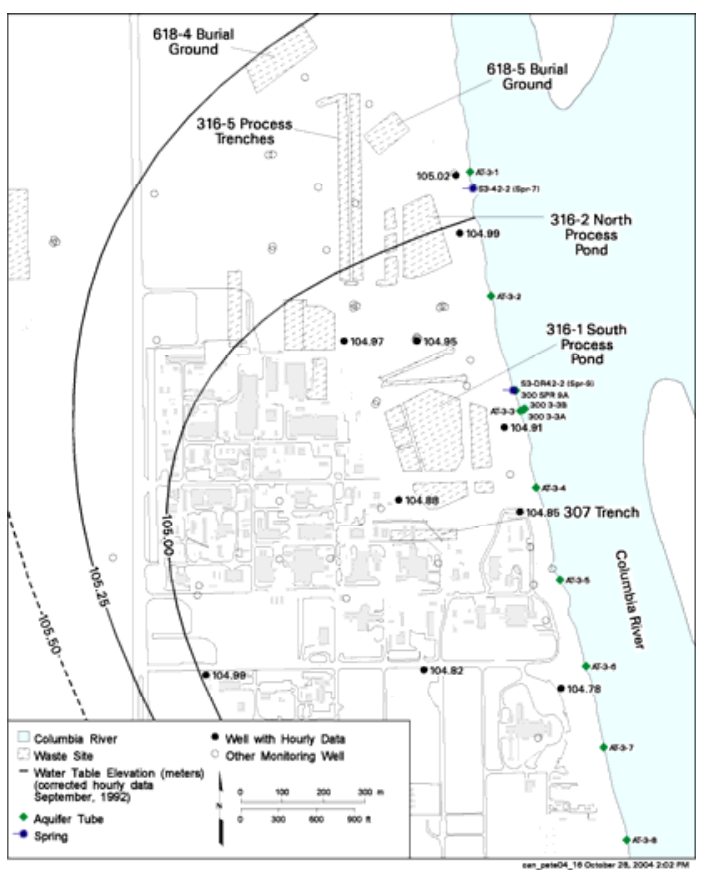

Figure 3.9. 300 Area Water-Table Elevation, September 1992 (Averaged Hourly Data)

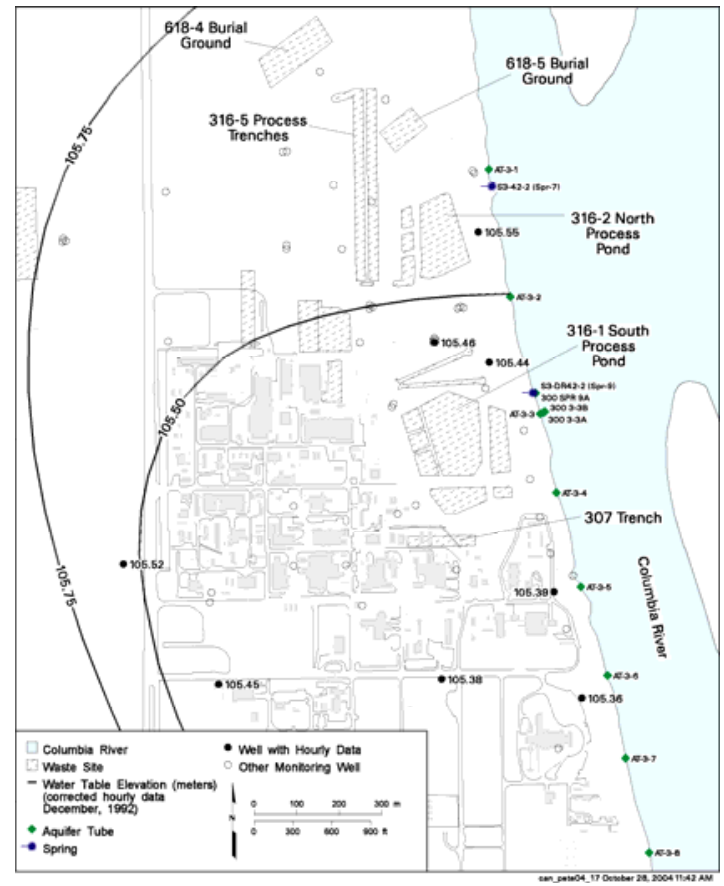

Figure 3.10. $\quad 300$ Area Water Table Elevation, December 1992 (Averaged Hourly Data) 
The fluctuating river stage causes corresponding fluctuations in water-table elevations but does not appear to have an appreciable affect on flow directions, at least when analyzed on a monthly scale.

Consequently, dispersal of contaminants from a particular source should follow a reasonably consistent path over the course of several years. The water-table maps indicate that the groundwater flow direction in the vicinity of the 300 Area process trenches, the last liquid waste disposal facility to receive uraniumbearing effluent, is generally to the south-southeast for all seasons.

Periodic reversal of the hydraulic gradient (i.e., directed inland from the river) occurs near the river when the stage is high, but this change is not readily apparent in the monthly averaged data. For example, during May 1992 (see Figure 3.7), the river stage generally increased throughout the month, yet the average flow pattern in the aquifer remained the same as for other months. The maps also suggest that along the shoreline to the south of the process trenches, river water may be continually entering the aquifer, flowing south along the shore, and then discharging back to the river. The highly transmissive Hanford unit is thicker along this section of shoreline, which would possibly enhance this exchange (see cross section in Figure 3.4).

The implication of having a fairly consistent long-term orientation of flow direction is that plume boundaries can be more accurately anticipated, especially when the source of the contaminant is also accurately known.

Because of the highly transmissive character of much of the uppermost hydrologic unit beneath the 300 Area (Hanford formation), the water table elevation responds quickly to fluctuations in stage of the adjacent Columbia River. Consequently, the hydraulic gradient steepness and orientation may vary dramatically over the short time periods associated with daily river fluctuations. This variability was visually represented by creating an animation of hourly contour maps for the 300 Area from water-level data collected between March 30 and September 26, 1992. The animation was prepared by M. P. Connelly at Westinghouse Hanford Company on January 11, 1993, and originally recorded as a video tape of the sequence as displayed on a computer screen. The original tape was subsequently digitized, and that file is available from R. E. Peterson. ${ }^{4}$ While the image quality of the animation is poor, the message regarding a highly dynamic water-table configuration is clear. Figure 3.11 shows the wells used to create the animation.

\subsection{Groundwater Flow and Transport Modeling}

Groundwater flow and uranium transport modeling are part of the planned work for the Phase III Feasibility Study for the 300-FF-5 Operable Unit (DOE 2005). Three-dimensional groundwater flow and transport models are being developed to predict the long-term characteristics of the uranium plume in 300 Area groundwater. When output from these models become available, they are expected to provide a significant amount of new information for the conceptual site model for uranium. The models will also play an important role in helping to select a remedial action alternative for the operable unit, and in evaluating potential remedial action technologies.

\footnotetext{
${ }^{4}$ R. E. Peterson, Pacific Northwest National Laboratory, Richland, Washington, 509-373-9020.
} 


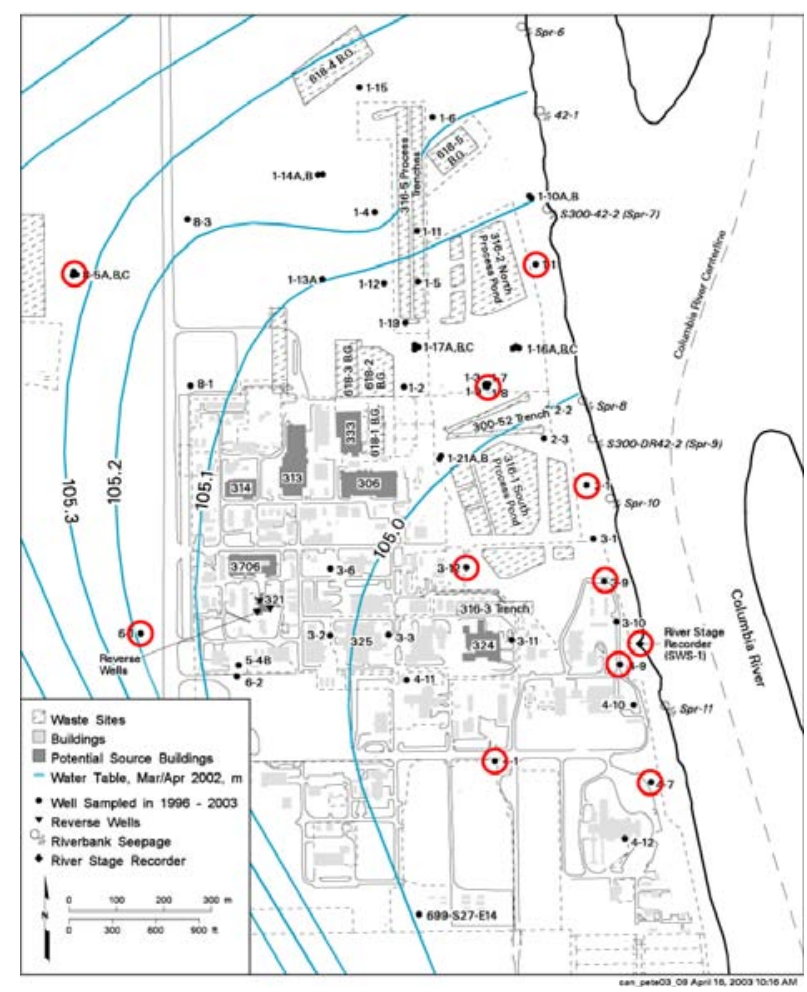

Figure 3.11. Wells Used in 1993 Animated Contour Maps

Model development is planned in three stages: (1) a hydraulic parameter estimation flow model using a historical time period with water level monitoring data available for estimating aquifer parameters and testing the model, (2) a prediction flow model with boundary conditions set based on potential future land use and other conditions, and (3) a uranium transport model that will use the current data on aquifer and vadose zone uranium concentrations, estimated hydraulic properties, and future conditions. All of these modeling efforts will use a common hydrogeologic framework, as described in Section 3.1. These models are combined saturated/unsaturated models for simulating the vadose zone and aquifer based on the STOMP code (White and Oostrom 2004, 2000; Oostrom et al. 2003).

\subsubsection{Hydraulic Parameter Estimation Model}

The hydraulic parameter estimation flow model is currently under development, with completion planned for the summer of 2005. The schedule for the additional numerical modeling tasks is shown in the work plan for the Phase III Feasibility Study (DOE 2005). The first model being developed is focused on estimating hydraulic properties of the Hanford and Ringold gravel units. Simulation results are being compared with extensive hourly water-level measurements collected from a network of 300 Area wells during the period December 1991 through March 1993 (Campbell 1994; Section 3.1.1 of this report). Three different hydraulic property distribution models are being tested: (1) single values for the main Hanford and Ringold gravel units, (2) main units sub-divided into zones with different properties, based on analysis of residuals), and (3) stochastic distribution of hydraulic properties, based on geostatistical analysis of physical property measurements from soil collected during well drilling in the area (e.g., Schalla et al. 1988; Swanson et al. 1992). A plan view of the grid that is currently being used for STOMP model simulations is depicted in Figure 3.12. 


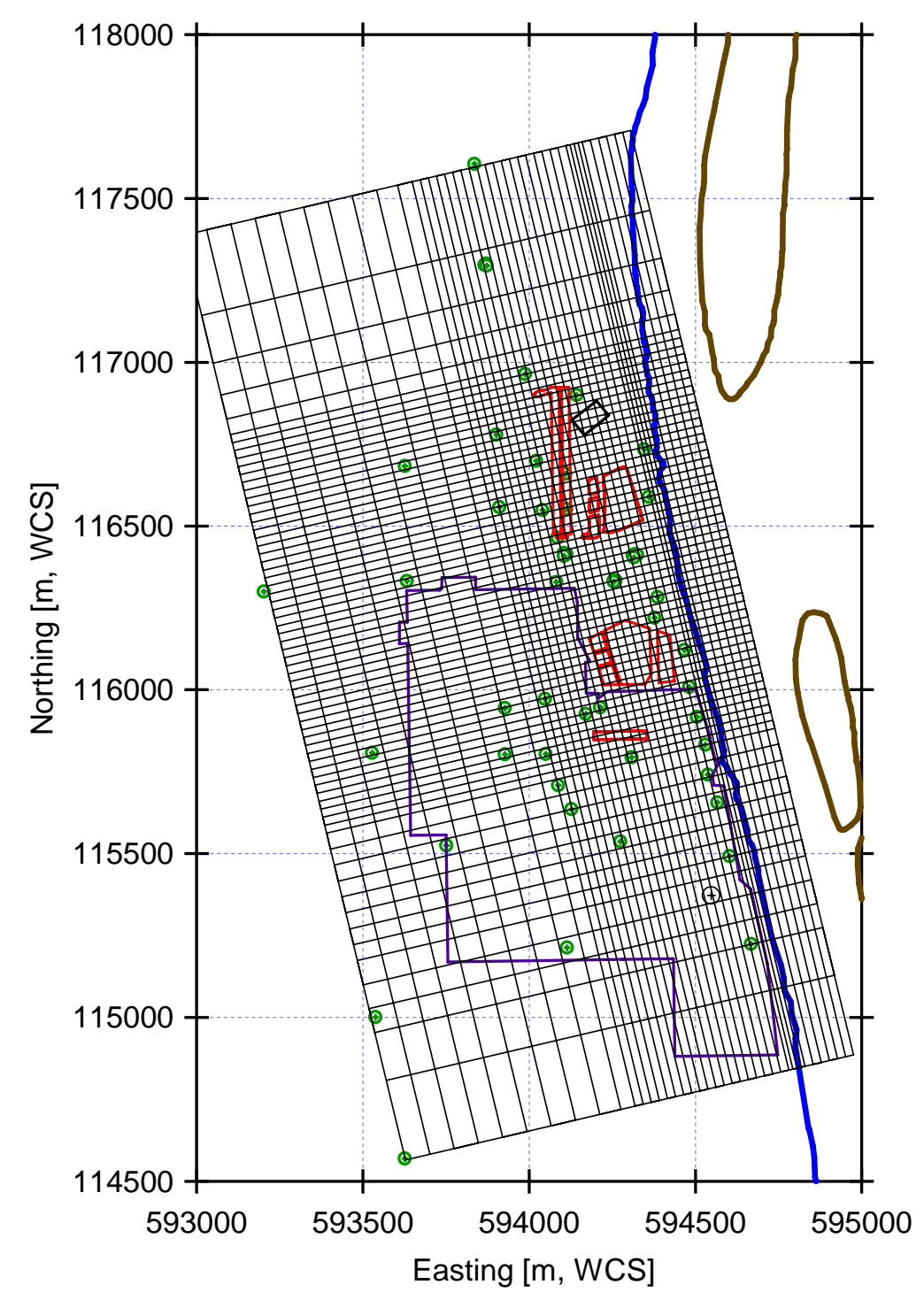

Figure 3.12. Plan View of STOMP Model Grid. Also shown are outlines of the 300 Area (purple lines), primary waste disposal areas (red lines), the western shoreline of the Columbia River (blue line), islands in the Columbia River (brown outlines), and miscellaneous wells (green circles). The black circled dot depicts the location of well 399-4-12.

The hydrogeologic framework developed using EarthVision for the 300 Area (see Section 3.1) is sampled at the three-dimensional finite difference STOMP model node locations to determine the hydrostratigraphic units for each node. Material properties include porosity, bulk density, hydraulic conductivity, and soil properties (e.g., Brooks-Corey parameters, residual saturation, relative permeability function). Initial values for hydraulic conductivity (1,500 meters [4,921 feet] per day for the Hanford formation and 150 meters [492 feet] per day for the Ringold Unit E gravels) were selected based on values used in the two-dimensional cross-section model. The hydraulic conductivity anisotropy was set at $0.1(\mathrm{Kz} / \mathrm{Kx})$. Initial values for porosity are $25 \%$ for the Hanford formation and $18 \%$ for the Ringold Formation. 
The east boundary of the model during the parameter estimation period is a specified head boundary condition set to hourly Columbia River stage measurements from a stage recorder in the 300 Area (SWS-1). The initial simulations use a constant average river gradient for nodes upstream and downstream of the SWS-1 along the boundary. Hydraulic heads are also specified along the western boundary of the model domain based on monthly average water-level measurements collected from three wells during the simulation period (wells 699-S29-E12, 699-S28-E12, and 399-8-2). An additional well is planned for the northwest corner of the model domain (see Figure 3.12) for the spring of 2005 to determine the hydraulic gradient along this portion of the model boundary and to refine the hydrostratigraphic units in this area with sparse well coverage. The north and south boundaries of the parameter estimation model are initially set to no-flow boundaries. Once estimates of hydraulic properties are narrowed, the hydraulic conductivities that are determined will be used to estimate water fluxes to be applied along these boundaries.

A flux of 55.4 millimeters (2.18 inches) per year is currently being applied for the upper boundary of the model to represent the long-term average natural groundwater recharge rate. This recharge rate is based on the analysis by Fayer and Walters (1995). During the parameter estimation period for the groundwater flow model (1991 to 1993), the discharges to the 316-5 process trenches are known to have impacted 300 Area groundwater. Estimated volumes of water discharged to the 316-5 process trenches are tabulated by Lindberg and Chou (2001, Table 3.2). The only known artificial withdrawal of groundwater that occurred during the model calibration period is pumping of well 399-4-12, which supplies water for the aquariums in the 331 Building.

\subsubsection{Prediction Flow Model}

This work element will develop a groundwater flow model for use in predicting future conditions, using the hydraulic properties determined from the parameter estimation process. This model will represent conditions in the future for recharge based on land use, other water sources (e.g., Richland Well Field), and water sinks (e.g., pumping). The three-dimensional finite difference STOMP grid and hydrostratigraphy will be the same as developed from the best-fit of the parameter estimation model. The east boundary of the model, which uses the Columbia River stage, would be cycled through the historical period since the operation of McNary Dam (1957) based on simulated river stages using a Columbia River model over this period (MASS1 see Richmond et al. 2000). Hydraulic heads for the west model boundary will be set based on relationships developed from analysis of the historical river stage and water-level measurements for wells along this boundary.

Simulated water fluxes and groundwater velocities will be calculated from the model used in the calibration process and in the prediction flow model. Values will be reported for instantaneous periods (e.g., hourly and daily) fluxes and longer term net and cumulative values. The results will focus on the aquifer/Columbia River interface on the east boundary of the model and will also provide model results of the regional groundwater fluxes into the model domain from the west boundary. The long-term net fluxes of groundwater to the river should be the same as the more-distant regional groundwater flux in the area. Groundwater velocities and stream lines with travel markers from the simulations will also be provided for selected time periods. Particle pathway (or stream lines) simulations utilizing the velocities from the groundwater flow model that provides for the best-fit to the observed hydraulic head data. The computational fluid dynamics add-in module for the Tecplot ${ }^{\mathrm{TM}}$ program will be used to generate visualizations of the particle tracks. 


\subsubsection{Uranium Transport Model}

The objective for the uranium transport work is to develop a model for use in predictions of future uranium concentrations under natural conditions and under the influence of various remediation technologies. The transport model will use the hydraulic properties estimated from the earlier flow modeling efforts described above and flow conditions determined for the prediction flow model. Initial conditions for uranium concentrations in the vadose zone and aquifer will be compiled to represent current plume conditions for the start of the uranium transport model simulations. In addition to developing the concentration initial conditions, other tasks involved in the development of the uranium transport model include developing a three-dimensional transport grid, determining the reactive transport mechanism to be used along with field-scale parameters appropriate for the three-dimension model, and running simulations for the identified MNA and remediation scenarios. These efforts are discussed briefly below.

Develop Uranium Initial Conditions. All available data on concentrations of uranium in the 300 Area vadose zone will be compiled and a three-dimensional conceptualization of the uranium distribution in the aquifer and vadose zone will be developed. For the aquifer, this effort will build on an initial representation of aqueous uranium concentrations based on groundwater monitoring data that was developed in 2003. It will also make use of geostatistical representations of uranium distributions in the aquifer that are being developed (Murray et al. 2004). For the vadose zone, chemical analysis of uranium concentrations in sediment samples collected from the 300 Area have been reported in Swanson et al. (1992), DOE (1994), Serne et al. (2002), and Zachara (2004). The total uranium in the aquifer and vadose zone will be calculated considering partitioning to soil. These sums will be compared to other available inventory estimates.

Construct Three-Dimensional Transport Grid. The three-dimensional uranium transport model will be developed from the three-dimensional flow model using the STOMP simulator. The new grid will be tested with conservative tracer simulations prior to use in uranium transport simulations.

Select/Implement Uranium Reactive Transport. This work element will assess the various uranium reactive transport mechanisms that are being studied at the laboratory scale and select one for use in the large-scale three-dimensional 300 Area flow and transport model. Reaction constants and other data requirements, such as concentrations of other important species, also need to be determined for use in the three-dimensional, field-scale simulations.

An equilibrium-based surface complexation model (SCM) has been developed to describe the sorption characteristics of uranium in 300 Area sediment (Davis et al. 2004). Although the SCM model has been shown to reproduce experimentally observed uranium sorption data quite well, in summarizing other results, Zachara et al. (2005) state that "U(VI) release from the sediment was found to be very slow and to require extensive water volumes for even partial removal of the sorbed U(VI) plume. U(VI) desorption was found to be a kinetic and not an equilibrium process." While the equilibrium-based SCM represents a significant improvement over simple Kd-based transport models, the SCM may not be entirely appropriate for regions in which rapid transients occur, such as the zone of interaction between the river and groundwater in the 300 Area. Zachara and co-workers are developing a kinetic, multi-rate model to describe their experimental results (Liu et al. 2004). The model uses a gamma distribution function to define the multiple rates. 
Future Prediction Uranium Transport Simulations. The objective for this work element is to use the appropriate transport model to evaluate natural attenuation processes and the performance of selected remedial action technologies. For each remedial action option, the appropriate model will need to be matched to the remedial option requirements. Natural attenuation will be simulated with the threedimensional uranium transport model, with initial concentrations in the aquifer and vadose zone set to values representing recent conditions. Long-term simulations will be conducted using groundwater flow conductions developed for the future prediction model. Some remedial options may require the threedimensional model for simulating processes such as complex pumping or injection strategies. Other remedial options may require simulations at a very fine spatial resolution (e.g., 1 meter [3.28 feet]), which would preclude the use of a large three-dimensional model for the entire 300 Area domain.

\subsection{Trends in Plume Parameters}

This section contains the results of a geostatistical analysis of uranium plume concentrations over time. The analysis was conducted to reveal trends in various plume parameters. Input values for this analysis are shown on the trend charts presented in the Appendix A. The time periods analyzed were: 1996 to 1998; 1998 to 2000; 2000 to 2002; and 2002 to 2004. Each time period is referred to by the midpoint year of the interval, i.e., 1997, 1999, 2001, and 2003.

\subsubsection{Geostatistical Methods to Characterize Plumes}

Geostatistical modeling and simulation of the spatial distribution of uranium concentrations were used to characterize the 300 Area uranium plume. Use of geostatistical methods has the advantage of greater objectivity when comparing representations of a contaminant plume for multiple time periods. A Monte Carlo approach was used to generate suites of realizations for the mass of uranium on a series of regular grids. Mass estimates were developed for several assumed plume thicknesses using Monte Carlo sampling of the estimated porosity distribution for Hanford formation sediment. Aggregate metrics were computed for the four time periods analyzed, including (a) total mass, (b) location of the center of mass, (c) area above the drinking water standard of $30 \mu \mathrm{g} / \mathrm{L}$, and (d) length of the Columbia River shoreline affected by the plume.

The geostatistical modeling included variogram analysis to define a mathematical model for the spatial continuity of the contaminant concentration data. The variogram is a measure of the average dissimilarity between pairs of points which are separated by a vector distance, as a function of that distance. Variables associated with geologic processes that vary spatially (e.g., contaminant concentrations within a groundwater plume), often display spatial continuity that can be identified by geostatistical analysis. If a variable exhibits spatial continuity, then points that are close to one another will have smaller differences and, therefore, lower variogram values than pairs of points that are separated by greater distances. In variogram analysis, models are fit to the experimental variograms that quantify the spatial continuity of the variable. Variogram models are required for geostatistical estimation (i.e., kriging) or simulation algorithms because it is rare that experimental variogram values will be available for all lag distances for which estimates or simulations may be desired. Spherical variogram models (Isaaks and Srivastava 1989) were fit to all variograms in this study. 
A geostatistical approach known as sequential Gaussian simulation was used to generate suites of realizations for the concentration of uranium on a regular grid. The sequential Gaussian simulation technique requires that the data follow a normal distribution. Because the raw uranium concentration data were not normally distributed, the sequential Gaussian simulation was performed on a normal score transform of the data. The normal score transform is a graphical transform that ensures the data fit a univariate normal distribution, while avoiding estimation bias problems that occur when using the logarithmic transform.

\subsubsection{Representative Concentrations Over Time for the Uranium Plume}

Representative uranium concentrations for the time periods were selected by reviewing all historical data for each 300 Area well, using the groundwater project's data viewer and evaluator (DaVE), which is a user interface with the Hanford Site groundwater database. Data deemed to be non-representative of long-term conditions were marked for exclusion from subsequent statistical calculations to determine average values. Minimum, maximum, and average values were calculated by DaVE for 2-year time intervals for each well, starting in 1974 and continuing to 2004. The averages (i.e., representative values) for each well, for each time period, are shown in Table 3.1. If no data existed for a well within a particular time interval, no representative value was assigned.

For the geostatistical analysis, the time period analyzed was initially limited to 1996 to 2004; subsequent work will extend the analysis back to 1986. Trend charts for each well were prepared that show (a) uranium results used, (b) non-detect results, (c) excluded results (i.e., outliers), and (d) the representative value assigned to each time interval (Appendix A). These charts were used to identify and modify obvious misrepresentations that might have occurred during the automated statistical summarizing of data.

The monitoring well locations where representative concentrations were available, and the concentration data class for each well and time period, are shown in Figure 3.13. The concentration characteristics for the four time periods are shown in Figure 3.14, using box plots. In a box plot, the longest bar represents the range in values; the top and bottom of the box represent the 75th and 25th percentiles of the data, respectively; the center of the notch represents the median value; and the vertical extent of the notch represents the $\sim 95 \%$ confidence interval for the median. A similar process was used to develop representative values for the water level in each well, for each time interval. The water-level elevation characteristics for the four time periods are shown in Figure 3.15, using box plots. 
Table 3.1. Representative Uranium Concentrations at Each 300 Area Well for Two-Year Intervals: 1974 to 2004

\begin{tabular}{|c|c|c|c|c|c|c|c|c|c|c|c|c|c|c|c|}
\hline Well Name & $\begin{array}{c}1974- \\
1976 \\
\end{array}$ & \begin{tabular}{|c|}
$1976-$ \\
1978 \\
\end{tabular} & $\begin{array}{c}1978- \\
1980 \\
\end{array}$ & $\begin{array}{c}1980- \\
1982 \\
\end{array}$ & $\begin{array}{c}1982- \\
1984 \\
\end{array}$ & $\begin{array}{c}1984- \\
1986 \\
\end{array}$ & $\begin{array}{c}1986- \\
1988 \\
\end{array}$ & $\begin{array}{c}1988- \\
1990 \\
\end{array}$ & \begin{tabular}{|c|}
$1990-$ \\
1992 \\
\end{tabular} & \begin{tabular}{|c|}
$1992-$ \\
1994 \\
\end{tabular} & $\begin{array}{c}1994- \\
1996 \\
\end{array}$ & $\begin{array}{c}1996- \\
1998 \\
\end{array}$ & $\begin{array}{c}1998- \\
2000 \\
\end{array}$ & $\begin{array}{l}2000- \\
2002 \\
\end{array}$ & $\begin{array}{l}2002 \\
2004 \\
\end{array}$ \\
\hline |399-1-1 & 132 & 79 & 23 & 24 & 27 & 28 & 23 & 21 & & & & & & & 44 \\
\hline 399-1-10A & & & & & & & 11 & 21 & 124 & 108 & 91 & 80 & 55 & 42 & 92 \\
\hline 399-1-11 & & & & & & & 58 & 74 & 102 & 30 & 23 & 79 & & 9 & 19 \\
\hline \begin{tabular}{|l|}
$399-1-12$ \\
\end{tabular} & & & & & & & 48 & 66 & 51 & 51 & 38 & 68 & 22 & 16 & 25 \\
\hline 399-1-13A & & & & & & & 14 & 14 & 21 & 6 & 7 & 14 & & & \\
\hline 399-1-14A & & & & & & & 18 & 18 & 19 & 6 & 6 & 16 & 8 & 6 & \\
\hline 399-1-15 & & & & & & & 8 & 9 & 5 & 5 & 4 & 7 & & & 6 \\
\hline 399-1-16A & & & & & & & 13 & 16 & 58 & 143 & 102 & 100 & 89 & 99 & 67 \\
\hline 399-1-17A & & & & & & & 65 & 162 & 176 & 17 & 221 & 405 & 130 & 79 & 55 \\
\hline \begin{tabular}{|l|}
$399-1-18 A$ \\
\end{tabular} & & & & & & & 4 & 5 & 5 & 4 & 5 & 6 & 6 & 6 & 6 \\
\hline 399-1-19 & & & & & & & 199 & 219 & & & 198 & 271 & & & \\
\hline 399-1-2 & & 41 & 11 & 28 & 26 & 30 & 17 & 26 & & 21 & 28 & 43 & 13 & 7 & 18 \\
\hline 399-1-21A & & & & & & & & & & 44 & 23 & 82 & 34 & 16 & 33 \\
\hline $399-1-3$ & 102 & 51 & 21 & 45 & 43 & 59 & 36 & 77 & & & 53 & & 144 & 82 & \\
\hline 399-1-4 & & 37 & 12 & 23 & 11 & 18 & 26 & 31 & & 5 & 12 & & & & \\
\hline 399-1-5 & & 44 & 14 & 25 & 19 & 29 & 37 & 62 & & 92 & 107 & 92 & 50 & & \\
\hline 399-1-6 & & 31 & 13 & 21 & 14 & 20 & 15 & 22 & & 15 & 9 & 12 & 8 & & 7 \\
\hline 399-1-7 & & & & & & & 38 & 70 & 57 & 110 & 131 & 289 & 143 & 79 & 66 \\
\hline 399-2-1 & & 77 & 18 & 11 & 11 & 11 & 13 & 13 & 18 & 39 & 69 & 178 & 242 & 169 & 77 \\
\hline $399-2-2$ & & 66 & 25 & 22 & 26 & 34 & 29 & 29 & & 98 & 141 & 296 & 274 & 175 & 70 \\
\hline $399-2-3$ & & 60 & 11 & 11 & 11 & 16 & 16 & 13 & & 42 & 133 & 68 & & & \\
\hline 399-3-1 & 71 & 66 & 28 & 15 & 12 & 18 & 12 & & & & 67 & 122 & 252 & 216 & \\
\hline \begin{tabular}{|l|}
$399-3-10$ \\
\end{tabular} & & 39 & 45 & 31 & 21 & 32 & 40 & 30 & 19 & 18 & 32 & 55 & 88 & 161 & 98 \\
\hline 399-3-11 & & 54 & 30 & 38 & 34 & 32 & 40 & 34 & & 29 & 67 & 66 & 40 & 24 & 35 \\
\hline 399-3-12 & & & & 50 & 39 & 61 & 46 & 31 & & 32 & 32 & 77 & 32 & 15 & 39 \\
\hline $399-3-2$ & & 17 & 15 & 18 & 15 & 18 & 13 & & & 21 & 22 & & & & \\
\hline 399-3-3 & & 22 & 12 & 15 & 11 & 21 & 14 & 9 & & 10 & 16 & 23 & 17 & 12 & \\
\hline 399-3-6 & & 4 & 10 & 14 & 14 & 20 & 18 & 14 & & & 25 & 38 & 16 & 11 & 16 \\
\hline 399-3-8 & & 60 & 27 & & & & & & & & & & & & \\
\hline 399-3-9 & & 101 & 35 & 25 & 17 & 17 & 24 & 20 & 18 & 26 & 22 & & & & \\
\hline $399-4-1$ & & 36 & 17 & 20 & 19 & 23 & 23 & 16 & 17 & 14 & 18 & 45 & 23 & 17 & 21 \\
\hline 399-4-10 & & 73 & 38 & 34 & 22 & 38 & 43 & 40 & & 58 & 42 & & & 94 & 94 \\
\hline 399-4-11 & & & & & & & 17 & 13 & 14 & 14 & 24 & 46 & 21 & 16 & \\
\hline \begin{tabular}{|l|}
$399-4-12$ \\
\end{tabular} & & & & & & & & & & 24 & 21 & 42 & 33 & 21 & 21 \\
\hline \begin{tabular}{|l|}
$399-4-7$ \\
\end{tabular} & & 86 & 45 & 43 & 35 & 46 & 49 & 38 & 34 & 45 & 43 & 49 & 71 & 68 & \\
\hline $399-4-9$ & & 54 & 34 & 24 & 26 & 30 & 39 & 33 & & 48 & 39 & 101 & 146 & 131 & 84 \\
\hline 399-5-1 & & 9 & 11 & 10 & 10 & 12 & 8 & 6 & & 6 & 5 & 11 & 8 & 7 & \\
\hline 399-6-1 & & 17 & 10 & 10 & 10 & 13 & 11 & 9 & & 9 & & & & & \\
\hline 399-8-1 & & 9 & 10 & 10 & 10 & 14 & 6 & 4 & 5 & 5 & 5 & 14 & 6 & 5 & \\
\hline $399-8-2$ & & 4 & 10 & 10 & 10 & 5 & 4 & 3 & 2 & & & & & & \\
\hline 399-8-3 & & 8 & 10 & 11 & 10 & 11 & 7 & 5 & 5 & 5 & & 14 & & & \\
\hline 399-8-4 & & & & 11 & 11 & 4 & 5 & 3 & & 3 & & & & & \\
\hline 399-8-5A & & & & & & & & & & 10 & 14 & 6 & 13 & 9 & 11 \\
\hline 699-S19-E13 & & 6 & 10 & 10 & 10 & 7 & 5 & 5 & 4 & 4 & 5 & & & & \\
\hline \begin{tabular}{|l|} 
699-S19-E14 \\
\end{tabular} & & & & & & & & & & 5 & & & & & \\
\hline 699-S27-E12A & & & & & & & & & & & & & 7 & 9 & \\
\hline 699-S27-E14 & & 10 & 10 & 10 & 10 & 9 & 8 & 5 & & & & 7 & 8 & 7 & 8 \\
\hline \begin{tabular}{|l|}
$699-S 29-E 12$ \\
\end{tabular} & & 6 & 10 & 10 & 10 & 11 & 5 & 3 & & & 4 & & 6 & & \\
\hline 699-S29-E16A & & & & & & & & & & 2 & 2 & 3 & 4 & 4 & 4 \\
\hline 699-S30-E15A & & 7 & 10 & 10 & 10 & 6 & 3 & 2 & 2 & 2 & 2 & & 4 & 4 & 4 \\
\hline
\end{tabular}



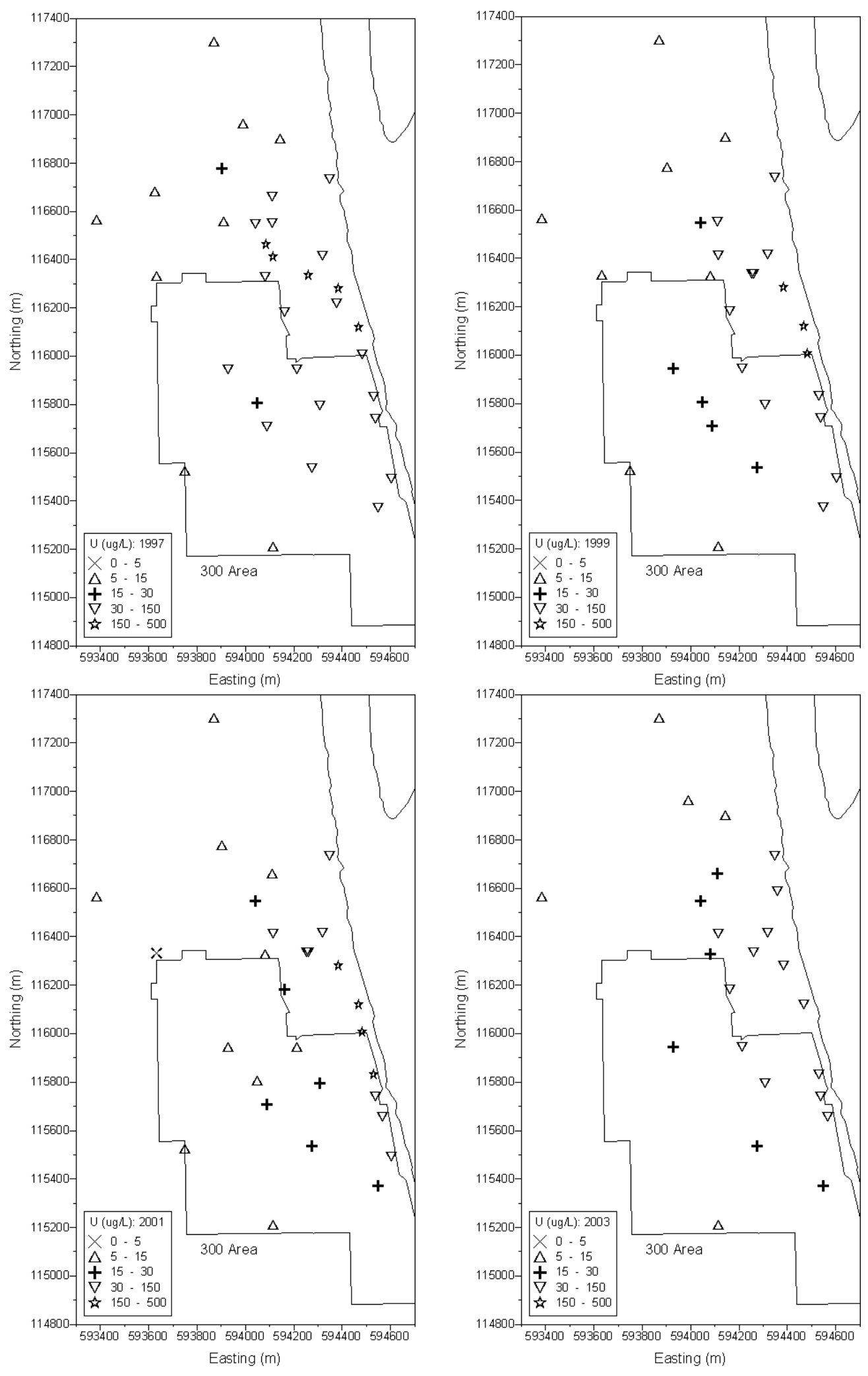

Figure 3.13. Maps Showing Locations for Representative Concentration Values and Data Classes for 300 Area Uranium Plume 


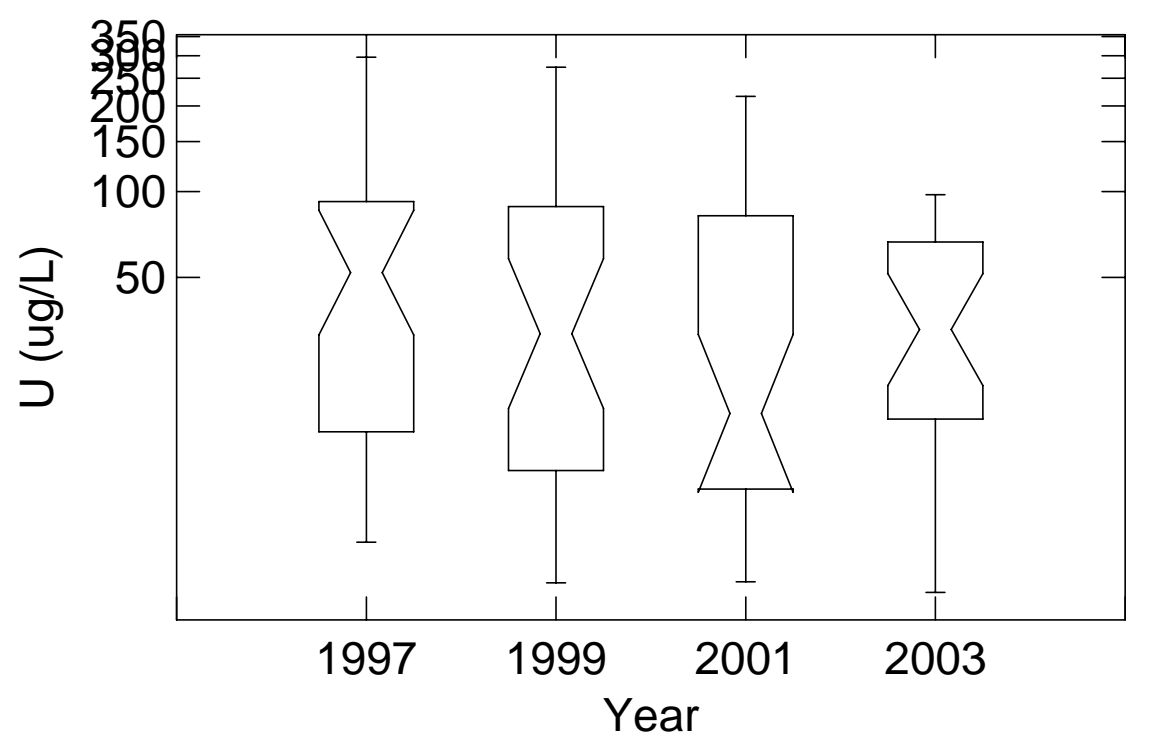

Figure 3.14. Box Plots for Uranium Concentration Data for Each Time Period

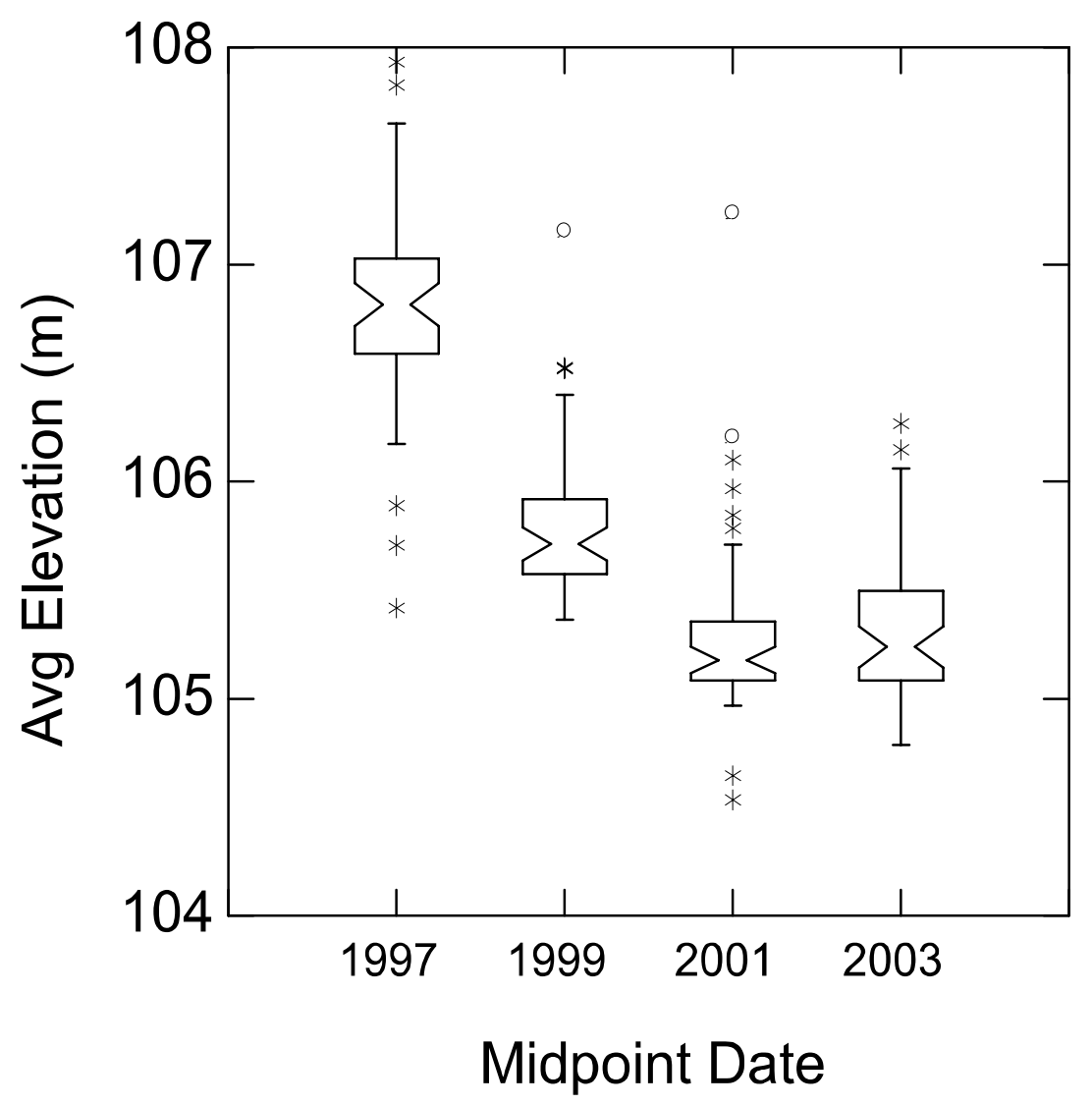

Figure 3.15. Box Plots Showing Average Water-Level Elevation 


\subsubsection{Principal Assumptions for the Geostatistical Analysis}

- The concentrations of uranium observed in groundwater at monitoring wells are representative of the level of uranium contamination in the aquifer.

- The concentration within the uranium plume is the same throughout the vertical extent of contamination; thicknesses of 2, 3, and 4 meters (6.56, 9.8, and 13 feet) were considered in the analysis.

- The dissolved uranium comprising the plume is primarily contained within saturated Hanford formation sediment. The effective porosity (i.e., pore space allowing groundwater movement) for saturated Hanford formation sediment was estimated to be $27 \%$.

- For the occasional fine-grained mud lenses that occur within the Hanford formation, effective porosity was considered to be zero (i.e., no significant amount of contamination is transported through these lenses because of their low hydraulic transmissivity).

\subsubsection{Results}

The distributions of median uranium concentrations for the four time periods, as determined from the suites of simulations, are shown in Figure 3.16. The shapes of the contoured areas shown in the figure are similar to contours of average values that are drawn by hand (see Figures 2.2 through 2.8). The probability that the concentration is above the drinking water standard for each time period is shown in Figure 3.17. The simulated total mass of dissolved uranium in the gridded area used for analysis of the 300 Area plume is shown for each time interval in Figure 3.18, using box plots. Total mass and variability, as judged by the range, maximum value, and 75th percentiles of the mass estimates shown in the box plots appear to have decreased since 1997.

The simulated plume area that exceeds the $30-\mu \mathrm{g} / \mathrm{L}$ drinking water standard is summarized in Figure 3.19, using box plots. The area decreases since 1997, but reverses trend for the most recent time interval. The simulated length of Columbia River shoreline impacted by the uranium plume, where it exceeds the drinking water standard, is summarized in Figure 3.20, again using box plots. The trend is similar to that for area, showing a decrease since 1997, but reversing trend for 2003.

The simulated values for (a) total dissolved uranium in the gridded area for three assumed thicknesses, (b) area of the plume exceeding the drinking water standard, and (c) average length of river shoreline impacted by the plume, are summarized in Table 3.2. The trends for each parameter reveal decreasing levels since 1997, although the rate of decrease appears to lessen during the most recent intervals. For the most recent time interval (2002 to 2004), simulation estimates are (a) total dissolved uranium in gridded area is 50 kilograms (110 pounds), assuming a 3-meter (9.84-foot) contaminated thickness, (b) area of the plume above the drinking water standard is $\sim 0.86$ square kilometers ( 0.33 square miles), and (c) the length of river shoreline impacted by the plume is $\sim 2,112$ meters (6,929 feet). 

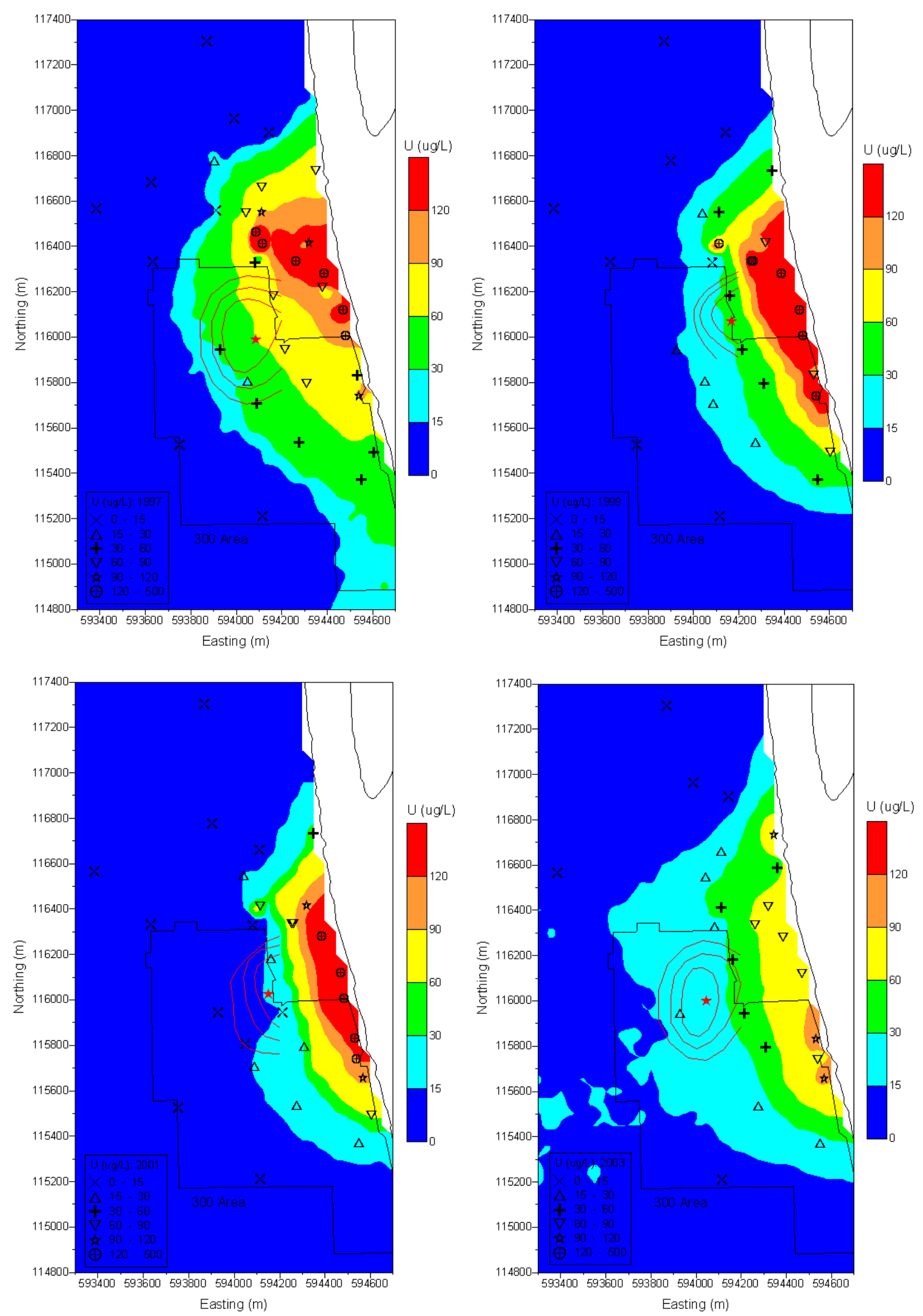

Figure 3.16. Median Concentration of Uranium in the 300 Area Plume for the Four Time Periods Simulated 

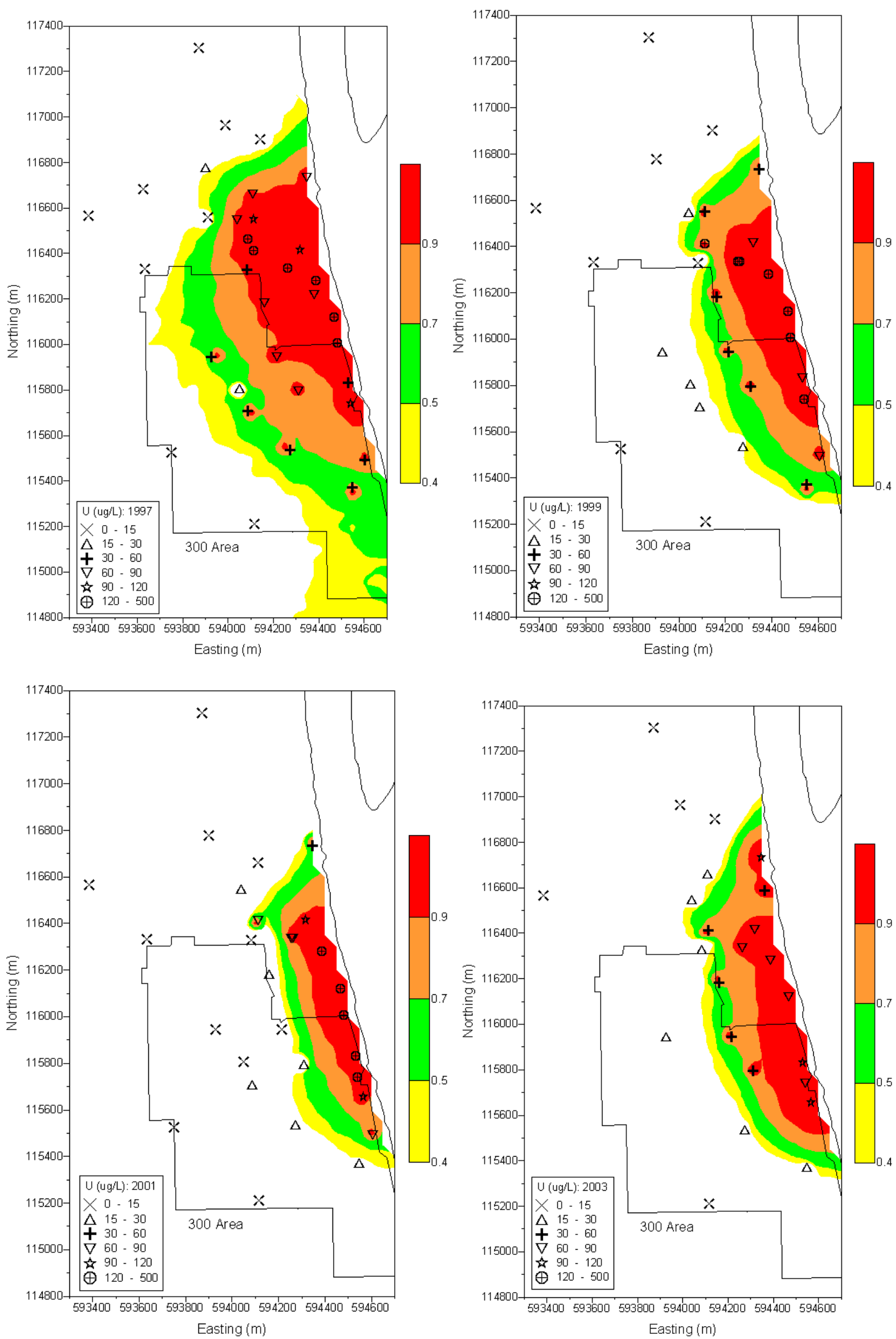

Figure 3.17. Probability of Exceeding the 30- $\mu \mathrm{g} / \mathrm{L}$ Drinking Water Standard for Uranium for the Four Periods Studied 


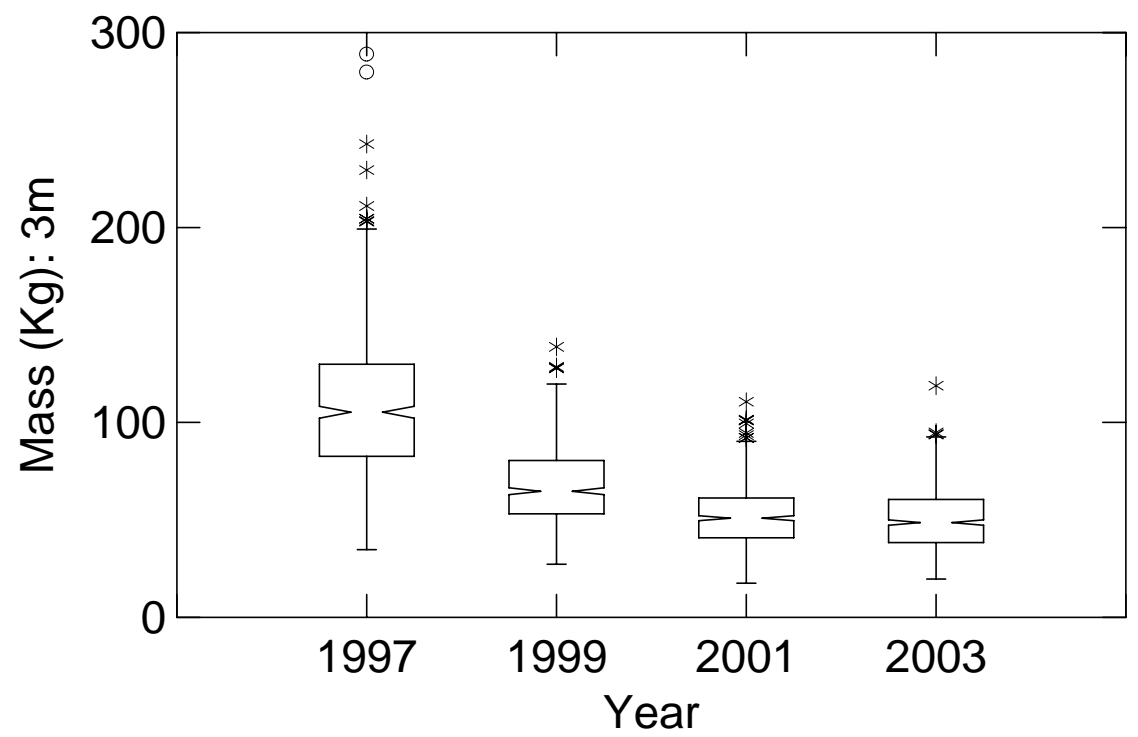

Figure 3.18. Box Plots Showing the Simulated Total Mass for the 300 Area Uranium Plume, Assuming a Thickness of 3 Meters

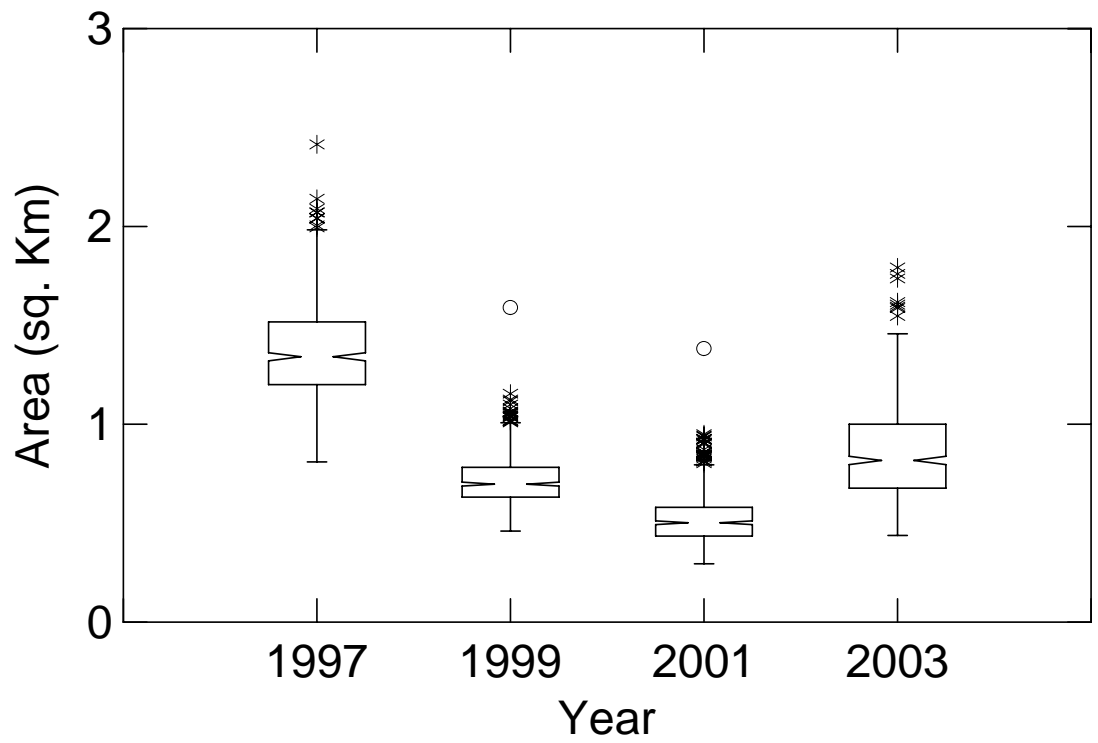

Figure 3.19. Box Plots of the Simulated Area Above the Drinking Water Standard for Each Time Period

\subsubsection{Discussion}

Analysis of the distribution of concentration data over time showed that most uranium plume parameters analyzed decreased with time for the four time periods, including the maximum concentration, the mean concentration, and the 75th percentile of the data (Table 3.3). For example, the maximum concentration decreased from 296 to $98 \mu \mathrm{g} / \mathrm{L}$ and the mean decreased from 77 to $41 \mu \mathrm{g} / \mathrm{L}$. However, the median uranium concentration and the 25th percentile showed a slightly different pattern with decreases from 1997 through 2001, but increases from 2001 to 2003. The median concentration decreased from 52 to 


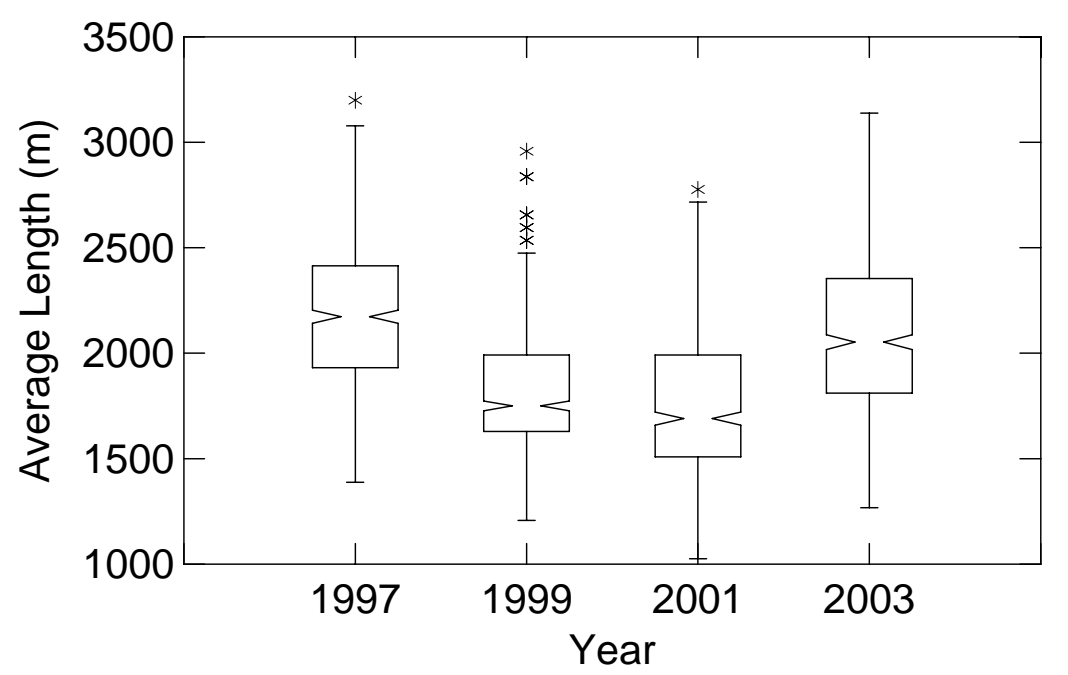

Figure 3.20. Box Plots of the Simulated Length of the Columbia River Shoreline Above the Drinking Water Standards for Each Time Period

$16 \mu \mathrm{g} / \mathrm{L}$ from 1997 through 2001, but then increased to $33 \mu \mathrm{g} / \mathrm{L}$ for the 2003 time period. One caveat for interpretation of the concentration data, based on this data set, is that the same wells were not sampled in all four time periods. This adds some uncertainty to the conclusions regarding trends.

A suite of 600 stochastic simulations of the uranium concentration were generated for each time period. The simulations were used to generate maps showing the median simulated value and the probability that the uranium concentration was above the $30 \mu \mathrm{g} / \mathrm{L}$ drinking water standard (Figures 3.16 and 3.17). The simulations were also used to calculate two statistics that are based directly on the mapped concentration values: (1) the area above the drinking water standard, and (2) the length of the Columbia River shoreline impacted by the plume at concentrations above the drinking water standard. The metrics for the area above the drinking water standard and the length of the shoreline above the drinking water standard tend to follow the pattern shown by the median concentrations in the raw data, i.e., an initial decrease in area above the drinking water standard from 1997 through 1999 and 2001, but then an increase in area above the drinking water standard from 2001 to 2003. The area above the drinking water standard in 2003 was similar to that seen in 1999, but not as high as the levels seen in 1997.

The stochastic simulations of concentration were transformed to Monte Carlo estimates for the mass of dissolved uranium in each grid cell. The mass estimates were based on three assumed plume thicknesses: 2, 3, and 4 meters (6.56, 9.8, and 13 feet), with a thickness of 3 meters (9.8 feet) being considered most representative. By summing over all grid cells in the study area, this process provided estimates for the mass of dissolved uranium for each time period, along with a measure of the uncertainty in those estimates. The mass estimates indicate substantial decreases in the mass of uranium from 1997 through 1999 and 2001 (i.e., from 108 to 52 kilograms [238 to 115 pounds), with the estimated mass remaining about the same in 2003 (50 kilograms [110 pounds]) as it was in 2001. Note that these values are for dissolved uranium in the entire gridded area, which is larger than the contaminant plume and includes natural background uranium. The mass estimates are therefore likely to be higher than estimates developed by other methods (see Chapter 2 on current characteristics). 
Table 3.2. Mass, Area, and Length of Impacted Shoreline for 300 Area Uranium Plume

\begin{tabular}{||c|c|c|c|c|c||}
\hline \hline & $\begin{array}{c}\text { Total Uranium } \\
\text { Mass: } 2 \mathrm{~m} \\
\text { Thickness } \\
(\mathrm{kg})\end{array}$ & $\begin{array}{c}\text { Total Uranium } \\
\text { Mass: } 3 \mathrm{~m} \\
\text { Thickness } \\
(\mathrm{kg})\end{array}$ & $\begin{array}{c}\text { Total Uranium } \\
\text { Mass: } 4 \mathrm{~m} \\
\text { Thickness } \\
(\mathrm{kg})\end{array}$ & $\begin{array}{c}\text { Area of Plume } \\
\text { Exceeding } \\
\text { Drinking } \\
\text { Water Standard } \\
\left(\mathrm{km}^{2}\right)\end{array}$ & $\begin{array}{c}\text { Average } \\
\text { Length of } \\
\text { Impacted } \\
\text { Shoreline } \\
\text { (m) })\end{array}$ \\
\hline \hline 1996 to 1998 & 73 & 108 & 137 & 1.37 & 2,173 \\
\hline 1998 to 2000 & 48 & 67 & 81 & 0.71 & 1,826 \\
\hline 2000 to 2002 & 39 & 52 & 61 & 0.53 & 1,760 \\
\hline 2002 to 2004 & 38 & 50 & 58 & 0.86 & 2,112 \\
\hline
\end{tabular}

Table 3.3. Statistical Summary of Representative Uranium Concentrations for Each Time Period

\begin{tabular}{|l|c|c|c|c||}
\hline \multicolumn{1}{|c|}{ Average Uranium $(\mu \mathrm{g} / \mathrm{L})$} & 1997 & 1999 & 2001 & 2003 \\
\hline \hline Mean & 77.39 & 64.49 & 53.40 & 40.50 \\
\hline Standard Error & 13.91 & 13.94 & 11.26 & 6.29 \\
\hline Median & 52.09 & 31.73 & 16.65 & 32.80 \\
\hline Standard Deviation & 81.12 & 77.59 & 61.66 & 31.47 \\
\hline Kurtosis & 2.05 & 1.62 & 0.57 & -1.14 \\
\hline Skewness & 1.65 & 1.58 & 1.28 & 0.56 \\
\hline Range & 290.54 & 269.28 & 211.75 & 93.74 \\
\hline Minimum & 5.86 & 4.22 & 4.25 & 3.90 \\
\hline Maximum & 296.40 & 273.50 & 216.00 & 97.64 \\
\hline Count & 34 & 31 & 30 & 25 \\
\hline Confidence Level of mean (95.0\%) & 28.30 & 28.46 & 23.02 & 12.99 \\
\hline \hline
\end{tabular}

\subsection{Geochemistry and Mobility of Uranium in 300 Area}

The persistence of a uranium plume in groundwater beneath the 300 Area has perplexed investigators who try to predict future conditions. This section discusses what is known about the geochemical controls on the mobility of uranium in the vadose zone and aquifer at the 300 Area. The new information is the product of research conducted during the past several years.

\subsubsection{Problem Statement}

Groundwater modeling work conducted during 1992 to 1993 indicated that the 300 Area uranium plume's maximum concentrations would decrease to the drinking water standard in 3 to 10 years, assuming no further input to groundwater from the vadose zone or other sources. As of 2003 to 2004, decreases in concentrations have occurred but not to levels at or below the drinking water standard. ${ }^{5}$ The

\footnotetext{
${ }^{5}$ The current drinking water standard is $30 \mu \mathrm{g} / \mathrm{L}$; the proposed standard in 1992 was $20 \mu \mathrm{g} / \mathrm{L}$
} 
assumption of no further input to the plume was an oversimplification, although the mechanisms responsible for such input are poorly understood.

Groundwater flow velocities in the 300 Area aquifer are relatively high compared to other Hanford Site regions. In the absence of re-supply of uranium from active liquid waste disposal activities, dispersion of the plume via discharge to the river would seem likely to happen in a relatively short period of time. This has not occurred, thus suggesting that geochemical processes play a significant role in retarding the movement of the plume and in maintaining uranium concentrations at certain levels.

\subsubsection{Geochemical Investigations}

Research involving the geochemistry and mobility of uranium in the subsurface at the 300 Area has been conducted during the past several years under the Hanford Site Remediation and Closure Science Project (formerly called Science and Technology) and DOE's Environmental Management Science Program. Principal objectives of this research include the following:

- Improve the conceptual model for the link between uranium in the vadose zone and the aquifer.

- Identify geochemical reactions controlling the solid/liquid distribution of uranium in the vadose zone and aquifer sediment, and measure descriptive thermodynamic and kinetic parameters.

- Develop a reactive transport simulator for use in predicting the future behavior of the groundwater plume.

This research is ongoing and continuing: the first objective is planned for completion by early spring 2005 to support the second 5-year review of the ROD for the 300-FF-5 Operable Unit. Information in Sections 3.4.3 and 3.4.4 is a preliminary description of that conceptual model. The second objective will be completed during 2006.

\subsubsection{Preliminary Geochemical Conceptual Model}

Waste from fuel fabrication, which contained copper and uranyl nitrate solutions, were typically acidic. Following discharge to infiltration ponds and trenches, the acidic nature of the waste promoted contaminant movement through the vadose zone and aquifer. Available groundwater data indicate somewhat lower $\mathrm{pH}$ values (i.e., more acidic) were present during operations in the 1970s and mid-1980s near the 300 Area process trenches. At times, the waste discharged to the ponds and trenches were neutralized by adding a base solution (e.g., sodium hydroxide). This was done to limit the migration of copper and uranium away from the waste disposal sites. Neutralization of waste caused precipitation and adsorption in the vadose zone (copper and uranium) and the aquifer (uranium). Over neutralization of the waste solutions occasionally occurred; this enhanced the mobility of the uranyl cation by creating uranyl carbonate complexes.

In the aquifer, a significant fraction of dissolved uranium adsorbed to fine-grained solids, which comprise a small fraction $(<8 \%)$ of the total sediment mass. During the peak fuel production years of the 1950s and 1960s, and again between 1975 and 1985, fuel-related liquid waste disposal caused extended periods when uranium concentrations in groundwater were elevated. These extended periods promoted diffusion of uranium into intraparticle sorption sites in aggregates of fine materials and weathered lithic 
fragments in the aquifer solids. Most, if not all, uranium that is sorbed to 300 Area sediment is hexavalent and present as the uranyl cation $\left(\mathrm{UO}_{2}{ }^{2+}\right)$, either in complexed, precipitated, or adsorbed forms.

The adsorption distribution coefficient $\left(\mathrm{K}_{\mathrm{d}}\right)$ for 300 Area sediment collected from several waste sites is highly variable. Estimates derived from this research indicate a range of 1 to 24 liters per kilogram for bulk sediment where the bicarbonate concentration approximates that of groundwater. Significantly higher distribution coefficients (e.g., >100 liters per kilogram) are observed for sediment containing precipitated uranium. Precipitated uranium generally occurs when the total uranium concentration of $<2$-millimeter- (0.08-inch-) size fraction of sediment exceeds 100 milligrams per kilogram.

Key variables that control the magnitude of the distribution coefficient include $\mathrm{pH}$; concentrations of calcium, bicarbonate, and uranium; $\mathrm{CO}_{2(\mathrm{~g})}$ partial pressure; aqueous speciation of uranium; mineral composition of solids; and sediment texture. The SCM developed as part of this research allows estimation of the distribution coefficient as a function of these variables for sediment and conditions where adsorption/desorption, and not precipitation/dissolution, is the primary retardation mechanism. The SCM may also be used directly in a reaction-based solute transport model, such as the Pacific Northwest National Laboratory STOMP code. The SCM predicts that the distribution coefficient will increase markedly (e.g., up to 10 times or more greater retardation) when low-carbonate water, such as river water, comes in contact with aquifer solids that are contaminated by the uranyl cation.

Uranium associated with sediment, whether in the form of precipitates or as adsorbed material, was released very slowly during tests on 300 Area samples, for several reasons:

- A significant fraction of sorbed uranium in near-surface sediment exists as a co-precipitate with calcite. (Note: Calcite precipitation was enhanced at the disposal sites when the acidic effluent was neutralized). Precipitates containing copper and phosphate Deeper in the vadose zone, a precipitate with copper and phosphate is observed. Solid carbonates dissolve slowly in the vadose zone and aquifer because the porewaters and groundwater are at or near thermodynamic saturation with calcite (assuming they are not mixed with river water in the zone of interaction near the river). The uranium incorporated in copper precipitates is slow to dissolve at the current $\mathrm{pH}$ of the 300 Area vadose zone.

- Adsorbed uranium occurs in phyllosilicate aggregates of waste and detrital origin that contain poorly crystalline iron oxides. Adsorption occurs to the surfaces of both clay minerals and iron oxides within the aggregates. The entrance and exit pathways to these aggregates are highly tortuous, and many pore volumes of liquid are required to leach the uranium from these aggregates.

The desorption/dissolution rates for uranium in the leaching experiments were controlled by the total sorbed uranium concentration, bicarbonate concentration in the leaching fluid, and by the fluid contact time. Under conditions that are assumed to approximate in situ (i.e., field) conditions, the maximum observed uranium concentration in porewater contacting uranium contaminate sediment was $95 \mu \mathrm{g} / \mathrm{L}$ for four samples studied from the deep vadose zone. Additional studies on this subject are underway. The observed half-lives for uranium adsorption/desorption and dissolution reactions were quite variable and material dependent but generally exceeded 50 hours. Uranium concentrations in groundwater may, therefore, be kinetically controlled and not at equilibrium.

Sorption/desorption kinetics are strongly influenced by the in situ sediment texture and depositional structures. Before laboratory results can be used to produce accurate predictions of transport in the field, 
additional work on scaling experimental results to field conditions must be accomplished. The experimental work performed to date has been on the <2.0-millimeter- (0.08-inch-) diameter fraction of sediment samples collected at only four waste site locations in the 300 Area. The $<2.0$-millimeter(0.08-inch-) size fraction represents $\sim 8 \%$ of the total sediment mass; $~ 75 \%$ of the total sediment mass is made up of smooth, rounded, cobbles with diameters $>12.5$ millimeters ( 0.49 inch).

\section{Implications of Research Results to the 300 Area Uranium Plume}

Residual uranium in the vadose zone beneath the remediated north and south process ponds shows no consistent trend with depth. It is estimated that $\sim 38 \%$ of the residual, sorbed uranium may be accessible to dissolution/desorption, but accessibility is highly variable because of heterogeneity in sediment characteristics and the chemical nature of sorbed uranium. Residual uranium exists in precipitated and adsorbed forms in sediment. Demarcation of these forms is difficult. Adsorbed uranium predominates in sediment with relatively lower amounts of total uranium (i.e., $<25$ ppm).

Experimental results suggest that the vadose zone beneath the remediated process ponds remain potential sources for uranium in groundwater, i.e., residual uranium is present and potentially mobile. Sorbed uranium in the lowermost vadose zone (i.e., capillary fringe region associated with the water table) is likely to exist in some areas and may also contribute to recharging the groundwater plume for many years. The remobilizing process is facilitated by fluctuations in water-table elevation-fluctuations that are caused by cycling of the Columbia River stage. The contribution associated with natural recharge is believed to be relatively small compared to the net flow of groundwater to the river. This would tend to reduce the impact of contaminated vadose zone pore water on groundwater quality. However, the situation might be different where much higher than expected aqueous concentrations were present in the vadose zone and/or there existed much more widespread contamination of the vadose zone than currently expected.

The degree of adsorption of uranium is partially controlled by the bicarbonate concentration of the interstitial fluids in sediment. Adsorption is less when bicarbonate concentrations are high (i.e., uranium is more mobile when more bicarbonate is present). The bicarbonate concentration of groundwater is significantly higher than that of river water. The infiltration of river water during high river stages lowers groundwater bicarbonate concentrations and increases the potential for uranium to adsorb onto near-river aquifer solids. This process is then reversed during periods of low river stage, i.e., the relatively higher concentrations of adsorbed uranium will desorb back into higher bicarbonate-containing groundwater that approaches the river, thus increasing near-river groundwater concentrations. A complex environment for adsorption/desorption cycling exists in the zone of interaction between groundwater and river water, where layering and mixing of the two water types occurs, along with highly irregular timing and duration of river stage cycles.

Experimental results indicated that uranium is adsorbed by vadose zone and aquifer solids more strongly than previously recognized. Travel times for adsorption/desorption fronts to move through the aquifer may be longer than previously estimated, perhaps by a factor ranging between 2 and 5 . Reactive transport modeling is underway to quantify these effects. Because significantly different uranium sorption properties were observed for sediment associated with the two sites sampled (north and south process ponds), reactive transport models developed to predict future plume conditions will need to include provisions to accommodate the strong heterogeneity in sediment characteristics that is present throughout the 300 Area. 
Selecting appropriate uranium transport codes for predicting plume behavior will also have to balance the rate of groundwater movement (advective flow) with the rates of exchange between aquifer-sorbed and dissolved uranium. Predicting how long the plume will persist above a certain concentration will continue to have uncertainty because of the very limited information on the sorbed uranium inventory in the vadose zone and aquifer.

\subsection{Key Aspects of Conceptual Model for 300 Area Uranium Plume}

The conceptual model for the uranium plume in the 300 Area has been updated with new information since the initial remedial investigation was performed in the early 1990s (DOE 1995). The new information comes from the accumulation of $\sim 12$ additional years of groundwater monitoring, laboratory investigations involving sediment contaminated by uranium, and computer simulation of groundwater movement and uranium transport. Descriptions of the hydrogeologic framework, groundwater flow characteristics, and controls on contaminant mobility have been updated, as appropriate, with emphasis on new information. Because some of the activities associated with enhancing the conceptual model are still in progress, periodic updates to the conceptual model are expected as new results and conclusions evolve.

Trends in Plume Characteristics. The principal causes for variability in uranium plume distribution patterns since the early 1990s are (a) removal of contaminated soils from the 300 Area process trenches, (b) cessation of liquid waste disposal to the ground, (c) large-scale source excavation activities, and (d) an unusually high water table during 1996 and 1997. During the most recent years, variability in concentration patterns appears to have lessened, with evidence suggesting a gradual downgradient migration of the higher concentration portions to the river. The highest concentrations observed currently are along the shoreline and probably reflect the last significant input from the soil beneath former major waste sites, such as the 316-5 process trenches. In the absence of additional disturbances such as the four described above, it appears likely that the plume will continue to exhibit relatively stable characteristics, including a general decline in observed concentrations. The causes for variability in the future may be restricted to extended periods of unusually high water table conditions and the dynamic interaction between river water and groundwater, as the result of river stage fluctuations.

Some characteristics, such as the general shape and extent, of the 300 Area uranium plume have remained fairly constant during the 1990s in spite of the perturbations described in the preceding paragraph. Estimates for plume volume and mass of dissolved uranium for the period 1998 to 2004 suggest that values have declined since 1998, although they appear nearly constant for the most recent several years. A generalization regarding this period is that concentrations are stabilizing at a level that is somewhat higher ( $10 \mu \mathrm{g} / \mathrm{L})$ than the current drinking water standard of $30 \mu \mathrm{g} / \mathrm{L}$. This suggests that the plume has achieved some degree of equilibrium with the hydrologic environment, i.e., a quasi-balance is being approached between the influx of clean groundwater to the area, some unknown degree of re-supply to the plume via release of uranium sequestered on vadose zone and/or aquifer solids, and the loss of uranium from the plume via river discharge and withdrawal from a water supply well.

Geochemical Controls on Mobility and Concentrations. Because of the persistence of the mapped uranium plume, in spite of the highly transmissive aquifer and absence of active waste disposal activities, geochemical processes are believed to exert a strong influence on the extent of contamination and concentrations in groundwater. The mobility of uranium and controls on dissolved concentrations are influenced by the geochemistry of the original waste effluent, the receiving sediment, and the compo- 
sition of pore fluids, all of which vary widely in the 300 Area environment. Recent laboratory investigations have shown that considerable heterogeneity exists, even within a single waste site. This compositional and spatial variability necessitates complex computer codes to design models for predicting plume behavior.

One working assumption in the conceptual model is that concentrations in groundwater were much higher during the 1950s and 1960s than they are today. The groundwater monitoring record is limited for those years, but when comprehensive monitoring did start during the early 1970s, the results suggested higher levels in previous years. When the first uranium-bearing effluent releases impacted groundwater, presumably at concentrations higher than currently, some uranium would have sorbed onto aquifer solids. Under conditions of lower concentrations, the sorbed uranium would tend to desorb, though probably at lower rates. This process may explain the persistence in some areas of the plume of relatively constant concentrations, i.e., as clean groundwater moves into the 300 Area, uranium desorbs from aquifer solids to maintain a certain concentration level. This process would continue until the uranium sequestered on aquifer solids is exhausted.

A second working assumption in the conceptual model is that uranium in groundwater during the 1950s and 1960s may have been moved upward into the vadose zone during an unusually high watertable event (first suggested by Lindberg and Bond 1979). As the water table returned to normal, residual moisture containing uranium would have been left behind in an extended capillary fringe zone. Some uranium would have sorbed to sediment in this zone. During subsequent periods of high water table conditions (e.g., 1996 and 1997), this uranium then desorbs into groundwater that has concentrations lower than during the earlier event. There is ample evidence in the monitoring record to show that uranium concentrations at many wells increase when the water table is raised above typical elevations.

Some re-supply of the plume may also be occurring beneath former liquid waste disposal sites, suggesting that the lower vadose zone beneath these sites contains uranium that may be mobilized by water-table fluctuations and/or is being driven downward by infiltrating moisture from the surface. The portions of the mapped plume at the south end of the former 300 Area process trenches, and in the vicinity of the 307 trenches (not yet remediated), show relatively higher concentrations during high water-table conditions than in other portions of the plume.

As the groundwater plume approaches the Columbia River, the geochemical environment changes because of infiltrating river water. During high river stage, river water moves inland and mixes and/or layers with groundwater, creating a zone of interaction. Uranium concentrations are lowered because of dilution by the uranium-free river water. The diluted mixture ultimately discharges back into the river via riverbank springs and upwelling through the riverbed substrate. Because river water is lower in bicarbonate content than groundwater (bicarbonate content influences the rate at which uranium sorbs and desorbs onto sediment), an additional process may act to sequester uranium in the zone of 
interaction. At lower bicarbonate concentrations, the tendency is for greater sorption onto solids, i.e., less mobility of uranium than in pure groundwater. While this process is theoretically possible, its significance to plume behavior has not yet been established.

Implications for Technologies to Reduce Concentrations. The current goal for groundwater in the operable unit is to have uranium concentrations below the drinking water standard ( $30 \mu \mathrm{g} / \mathrm{L})$. This goal may be achieved by a combination of source removal activities, dispersal of the plume via natural groundwater flow, and use of engineering solutions. Several significant uncertainties in the conceptual model for uranium currently hinder the selection of an appropriate engineering solution:

- Groundwater and uranium mass balance for the aquifer beneath the 300 Area and rate of pore volume exchange.

- Inventory and mobility of uranium in the vadose zone beneath waste sites, the extended capillary fringe zone ("smear zone"), and on aquifer solids.

- Rates and mechanisms by which the inventory is re-supplying the observed plume.

The groundwater mass balance issue is being investigated by constructing a three-dimensional model of groundwater flow in the 300 Area. Model output will include estimates for the volume of water entering and leaving the region beneath the 300 Area, and will include a provision for the significant volume of water withdrawn at the 331 Building for aquarium supply. The capability to include uranium transport calculations will be added to the model, and will incorporate the best-available information on uranium sorption/desorption rates (DOE 2005). Output will provide additional insight on remaining source inventories, i.e., how much remains in the environment to be addressed by the engineered solution.

The inventory of uranium potentially sequestered in the extended capillary fringe and beneath former liquid waste disposal sites can be better estimated by additional field observations. A borehole investigation has been proposed that will, at a minimum, provide data on the vertical distribution of uranium in the lower vadose zone and capillary fringe. The number of boreholes and their spacing will be designed to produce representative values for the major portion of the plume and beneath suspected hot spots (e.g., southern end of former 316-5 process trenches and 307 trench).

Finally, the considerable laboratory research that has investigated the sorption/desorption characteristics of uranium and 300 Area sediment has revealed a great amount of new information (e.g., Serne et al. 2002; Zachara et al. 2005). Unfortunately, one finding is that the geochemically controlled rates associated with uranium mobility are highly variable because of the heterogeneous natures of the waste effluent and the receiving sediment. So unless the subsurface environment can be mapped at a level of detail that is likely to be impractical, predictions for uranium transport in the vadose zone and aquifer will always include a level of uncertainty that will introduce some subjectivity into subsequent decisions based on the modeling output. 


\subsection{Conceptual Model for Tritium at 618-11 Burial Ground}

Development of a detailed conceptual site model for the hydrogeology and contaminant distribution in the vicinity of the 618-11 burial ground is an essential element of evaluating of the future impact of the tritium plume on potential receptors, which include users of groundwater downgradient of the burial ground and ecological receptors in the Columbia River. The following sections provide a background for development of the conceptual site model, which formed the basis for the local scale groundwater flow and contaminant transport model being developed to support this effort.

\subsection{Hydrogeologic Conceptual Model}

The 618-11 burial ground and the Energy Northwest nuclear power plant complex are constructed on suprabasalt sediment of Miocene to Pleistocene age. The stratigraphic column includes in ascending order from oldest to most recent, the Columbia River Basalt Group, Ringold Formation coarse-grained facies of the Cold Creek unit, and Hanford formation. In addition, a thin, regionally discontinuous veneer of Holocene alluvium and eolian sediment overlies the principal geologic units. Lindsey (1995) describes the regional geology of the Hanford Site. The hydrogeologic description of the Hanford Site is provided in Hartman (2000).

The three-dimensional representation of major hydrogeologic units in the vicinity of the 618-11 burial ground was based on information from borehole logs (see borehole locations in Figure 4.1) and on knowledge of the depositional environment at the site. The hydrogeologic interpretation was also influenced by information on the movement of tritium from the burial ground. Hydrogeologic units were delineated to reflect differences in hydraulic properties of sediment such as effective porosity and hydraulic conductivity. These properties are related to sediment texture, which is a function of grain-size distribution, sorting, and degree of consolidation/cementation. In developing the model, an effort was made to identify major textural units that influence groundwater flow directions and contaminant transport.

\subsubsection{Sedimentary Geologic Units}

Major sedimentary units that overlie basalt in the vicinity of the 618-11 burial ground are, in ascending order, the Ringold Formation, the coarse-grained facies of the Cold Creek unit, and the Hanford formation (informal name). These are described below.

Ringold Formation. After the last major eruption of basalt, the fluvial-lacustrine Ringold Formation was deposited in generally east-west trending valleys by the ancestral Columbia River and its tributaries. Fluvial deposits of the Ringold Formation include gravel and associated sand and silt deposited by the migrating river system and overbank sand, silt, and clay deposits that resulted from flooding beyond the influence of the main river channels. The fluvial units are separated by relatively thick layers of laminated mud with minor sand that were deposited periodically when the river channels were blocked causing lakes to develop. 


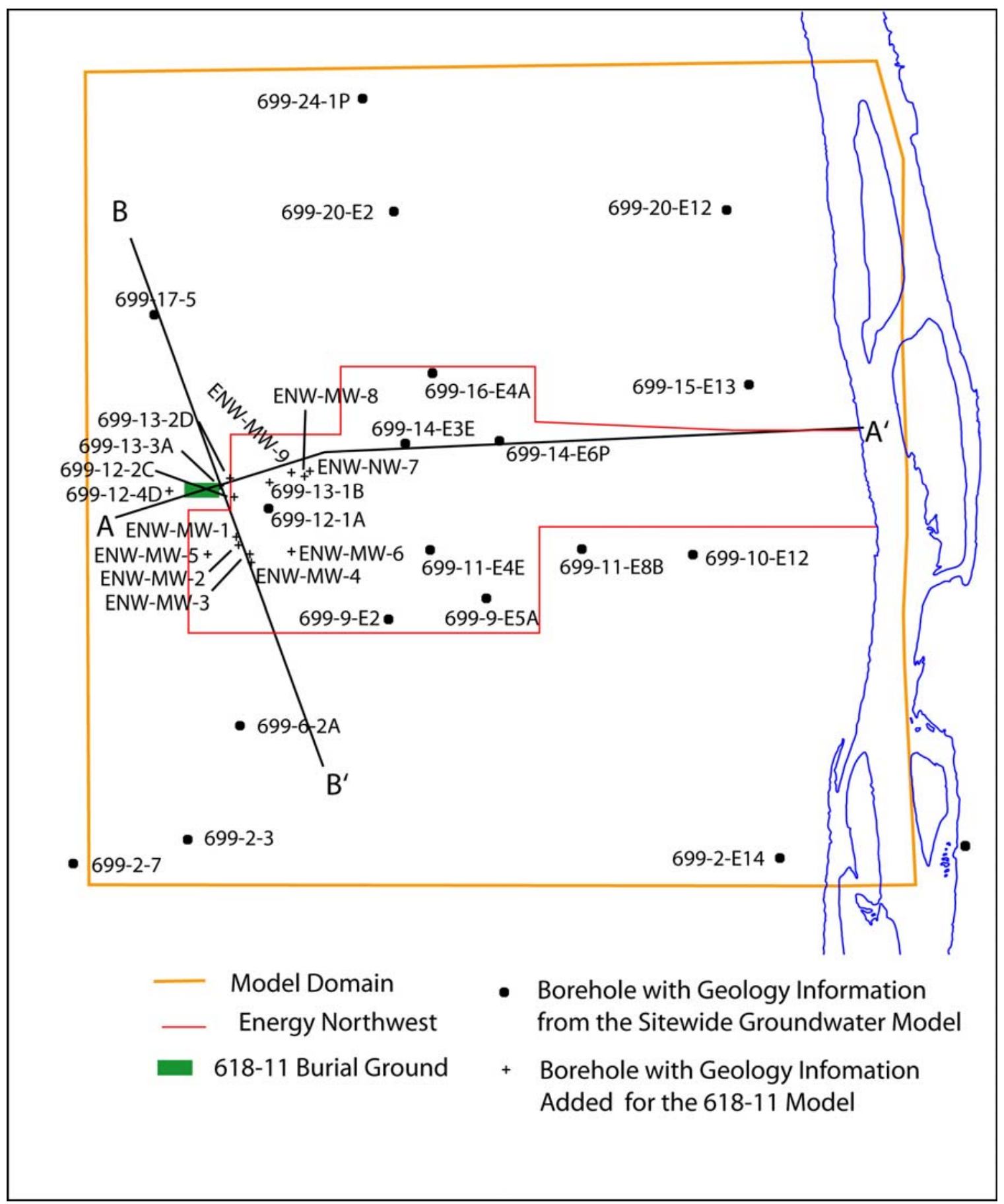

Figure 4.1. Location of Boreholes Used to Define the Hydrogeologic Conceptual Model

The alternating fluvial and lacustrine deposits within the Ringold Formation form distinct hydrogeologic units that have been identified in boreholes and correlated over distances of several kilometers. Identification of these hydrogeologic units was based on the facies associations defined by Lindsey (1995). These facies associations reflect differences in geologic characteristics and depositional environment that affect groundwater flow properties. Based on the distribution of dominant facies, three informal members of the Ringold Formation were defined by Lindsey (1995). 
Ringold sediments on the Hanford Site are dominated by the lowermost "member of Wooded Island." This member is divided into fluvial gravel dominated units designated, in ascending order, as A, B, C, D, and $\mathrm{E}$ by Lindsey (1995). The gravel and sand units are vertically separated by mud-dominated overbank and lacustrine deposits, including the extensive unit referred to as the Ringold lower mud that stratigraphically lies above gravel unit A.

The "member of Taylor Flat" lies stratigraphically above the unit E gravel and is dominated by fluvial sands and overbank fines. This member, referred to as the upper Ringold unit, has been removed from most of the central and southern portions of the Hanford Site by post-Ringold erosion. The upper Ringold unit is not present at the burial ground but is found between the burial ground and the Columbia River.

Cold Creek Unit. The coarse-grained facies of the Cold Creek unit consists of rounded clastsupported pebble- to cobble-sized gravel. It is generally coarser and less consolidated than the Ringold Formation and has a lower percentage of basalt clasts than the Hanford formation. It generally displays hydraulic conductivity that is greater than the underlying Ringold gravels, but less than the overlying Hanford formation. The coarse grained facies of the Cold Creek unit represents mainstream alluvial deposits of the ancestral Columbia-Clearwater-Salmon River system (DOE 2002c). Deposition occurred after a period of down-cutting into the Ringold Formation and may define the former course of the Columbia River southeastward through Gable Gap. The facies is narrow through Gable Gap and widens across the east-central portion of the Hanford Site, just north of the 618-11 burial ground. In some areas, some or all of the Cold Creek unit was subsequently removed during ice age flooding that deposited the Hanford formation.

Hanford Formation. The informally named Hanford formation sediment are generally coarser and less consolidated than the Ringold Formation. The Hanford formation was deposited by a series of cataclysmic floods that inundated the Pasco Basin during the last ice age, beginning as early as 2.5 million years ago (DOE 2002c). The last major flood sequence is dated at about 13,000 years ago by the presence of Mount St. Helens "S" tephra interbedded with the flood deposits. The number and timing of cataclysmic floods continues to be debated, but may be as high as 100 (DOE 2002c). The largest and most frequent floods came from glacial Lake Missoula in northwest Montana. Cataclysmic floodwaters entering the Pasco Basin quickly became impounded behind Wallula Gap, which was too restrictive for the volume of water involved. Floodwaters formed temporary lakes, which lasted only a few weeks or less.

The floods caused massive erosion of both earlier sediment and the basalt bedrock (DOE 2002c). They also resulted in rapid deposition of flood-borne sediment (Hanford formation) in low-lying areas. Cobbles, gravels, and coarse sand were deposited in the main flood channels with finer sand and silt being deposited on the fringes. Hanford formation sediment are continuous across the model area. However, in some places, the water table lies below the bottom of the Hanford formation.

Hanford formation sediment in the vicinity of the 618-11 burial ground predominately belong to the gravel dominated facies. Facies classifications and depositional environments of the Hanford formation are discussed in DOE (2002c). Gravel-dominated strata consist of coarse-grained sand and up to boulder sized clast-supported gravel. The gravels can have an open matrix with large pore spaces and very high permeability. Lenticular sand and silt beds are intercalated throughout the facies. Gravel clasts are generally dominated by basalt (50\% to $80 \%$ ). The gravel-dominated facies was deposited by high-energy floodwaters in or immediately adjacent to the main channel cataclysmic floodways (Reidel et al. 1992). 
Because of their coarse texture and lack of consolidation/cementation, the Hanford formation gravels are much more permeable than the Ringold sediment and tend to dominate groundwater flow where they exist below the water table.

Hydrogeologic Framework. Cross sections showing the hydrogeologic units relative to the 2001 water table are shown in Figures 4.2 and 4.3. Figure 4.4 shows the saturated thickness of the Hanford formation based on the 2001 water table. Data points used in defining the bottom of the Hanford formation are shown. Contours of the tritium plume measured in 2003 are also shown on the figure. The saturated Hanford formation gravels north of the burial ground appear to reflect a channel formed during the ice-age floods.

Beneath the 618-11 burial ground, the water table is below the bottom of the Hanford formation and in the less permeable Ringold Unit E gravel. In the vicinity of the burial ground, this unit is approximately 60 meters (196.8 feet) thick. However, during a field investigation conducted in FY 2001, ${ }^{1}$ a

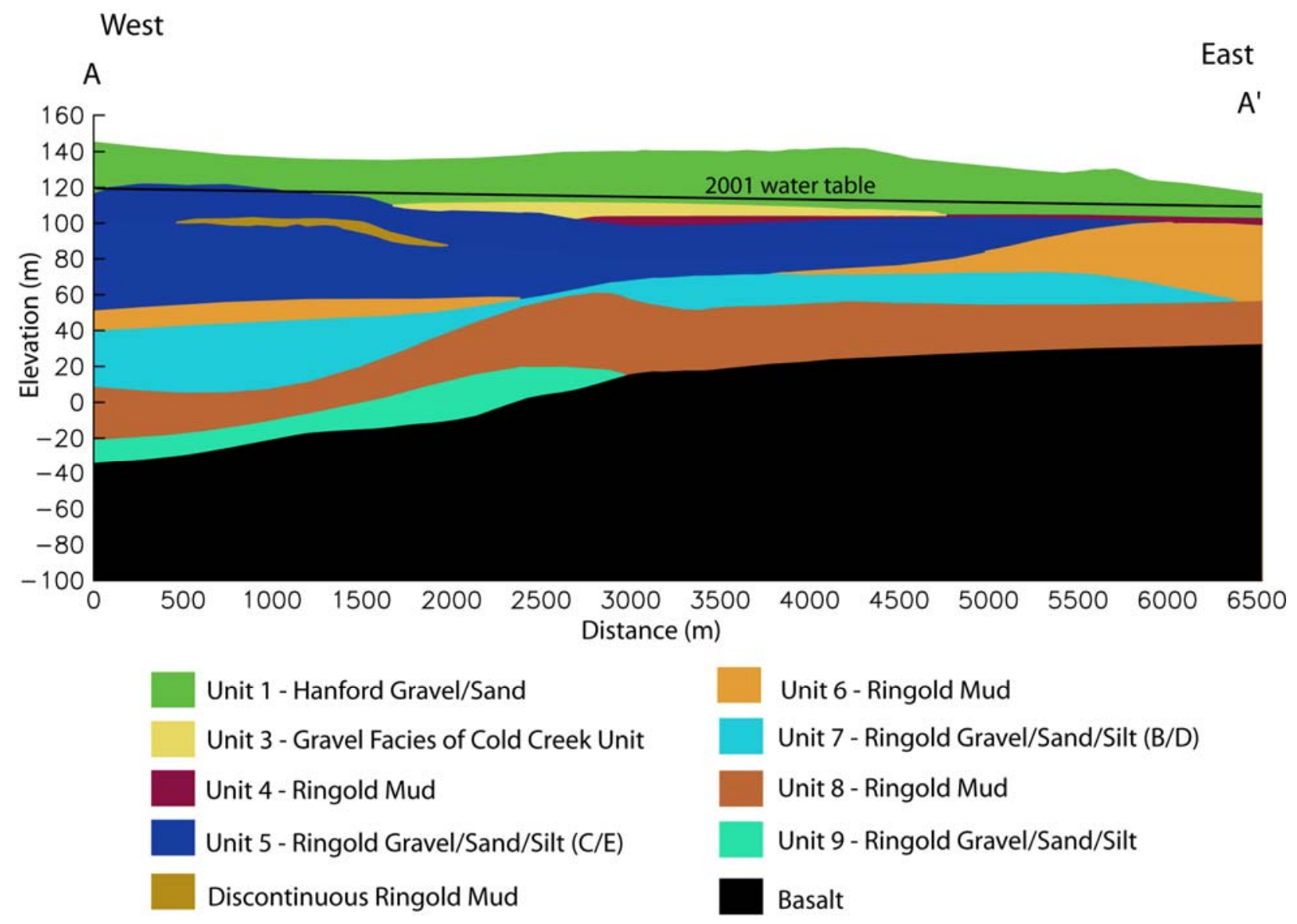

Figure 4.2. West-to-East Cross Section through the Model Showing Hydrogeologic Units (traverse shown on Figure 4.1)

\footnotetext{
${ }^{1}$ Letter report from Bechtel Hanford, Inc. to U.S. Department of Energy, Tritium Groundwater Investigation at the 618-11 Burial Ground, September 2001, dated September 28, 2001. Prepared by JV Borghese, WJ McMahon, and RW Ovink (CH2M HILL Hanford, Inc.) for Bechtel Hanford, Inc., Richland, Washington.
} 

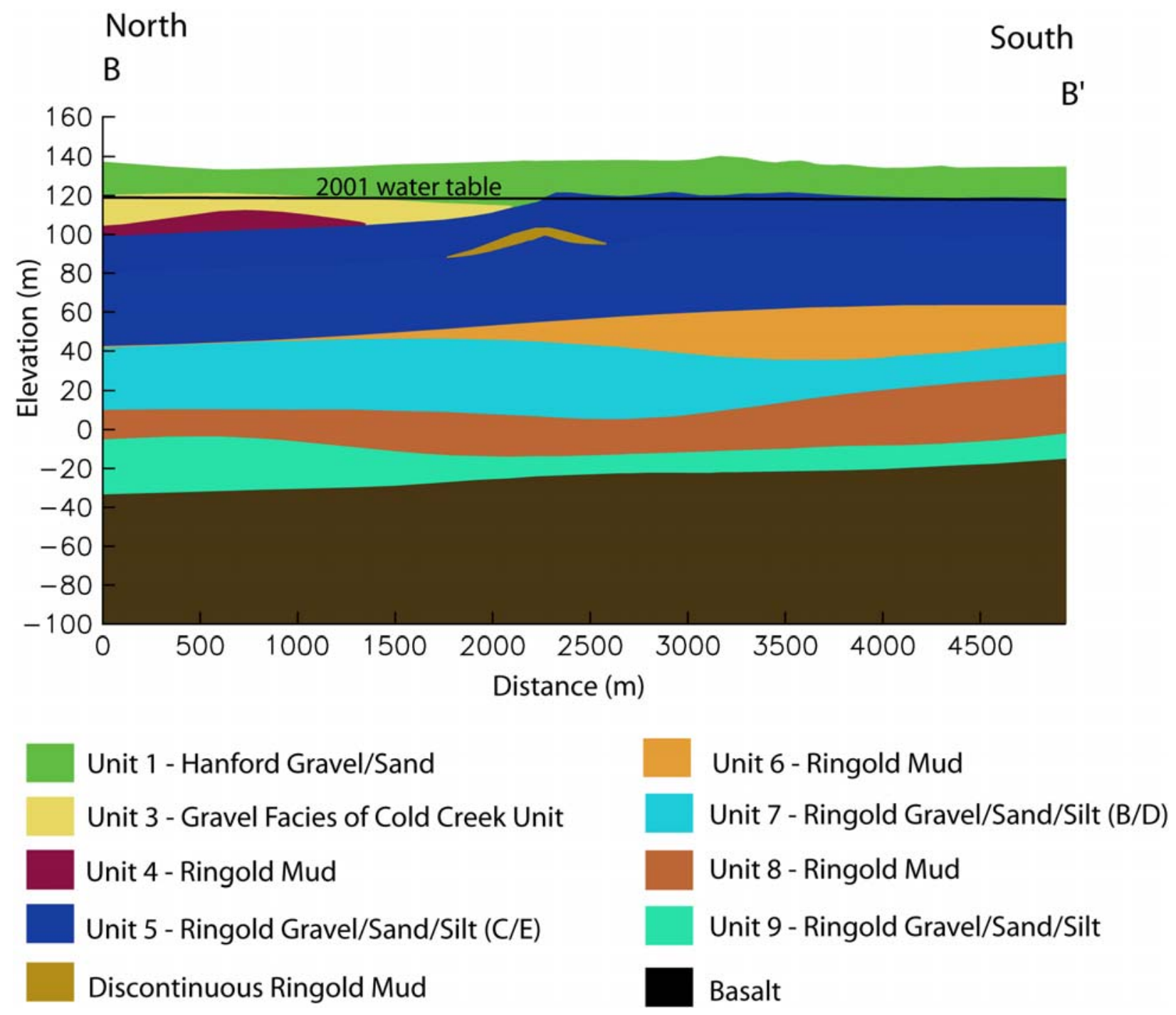

Figure 4.3. North-to-South Cross Section through the Model Showing Hydrogeologic Units (traverse shown on Figure 4.1)

Ringold mud unit was encountered during the installation of well 699-13-2D at a depth of approximately 14 meters (46 feet) below the water table. Because relatively few wells in this area are deep enough to encounter this unit, the areal extent of this feature is not well characterized. Based on geologic logs from other wells in the area, two of which show a similar feature (wells 699-13-1A and 699-13-1B), this mud unit is thought to be a relatively discontinuous feature (see Figures 4.2 and 4.3).

\subsubsection{Hydraulic Properties of Hydrogeologic Units}

Hydraulic properties including both horizontal and vertical hydraulic conductivity $\left(\mathrm{K}_{\mathrm{h}}\right.$ and $\left.\mathrm{K}_{\mathrm{v}}\right)$, storativity $(\mathrm{S})$, and specific yield $\left(\mathrm{S}_{\mathrm{y}}\right)$ are an important component of the hydrogeologic conceptual model. Hydraulic conductivity controls the rate of groundwater flow through a unit thickness of the aquifer at a given hydraulic gradient. Storativity and specific yield determine the change in water-table elevation that will occur in response to a change in the volume of water stored in the aquifer. 


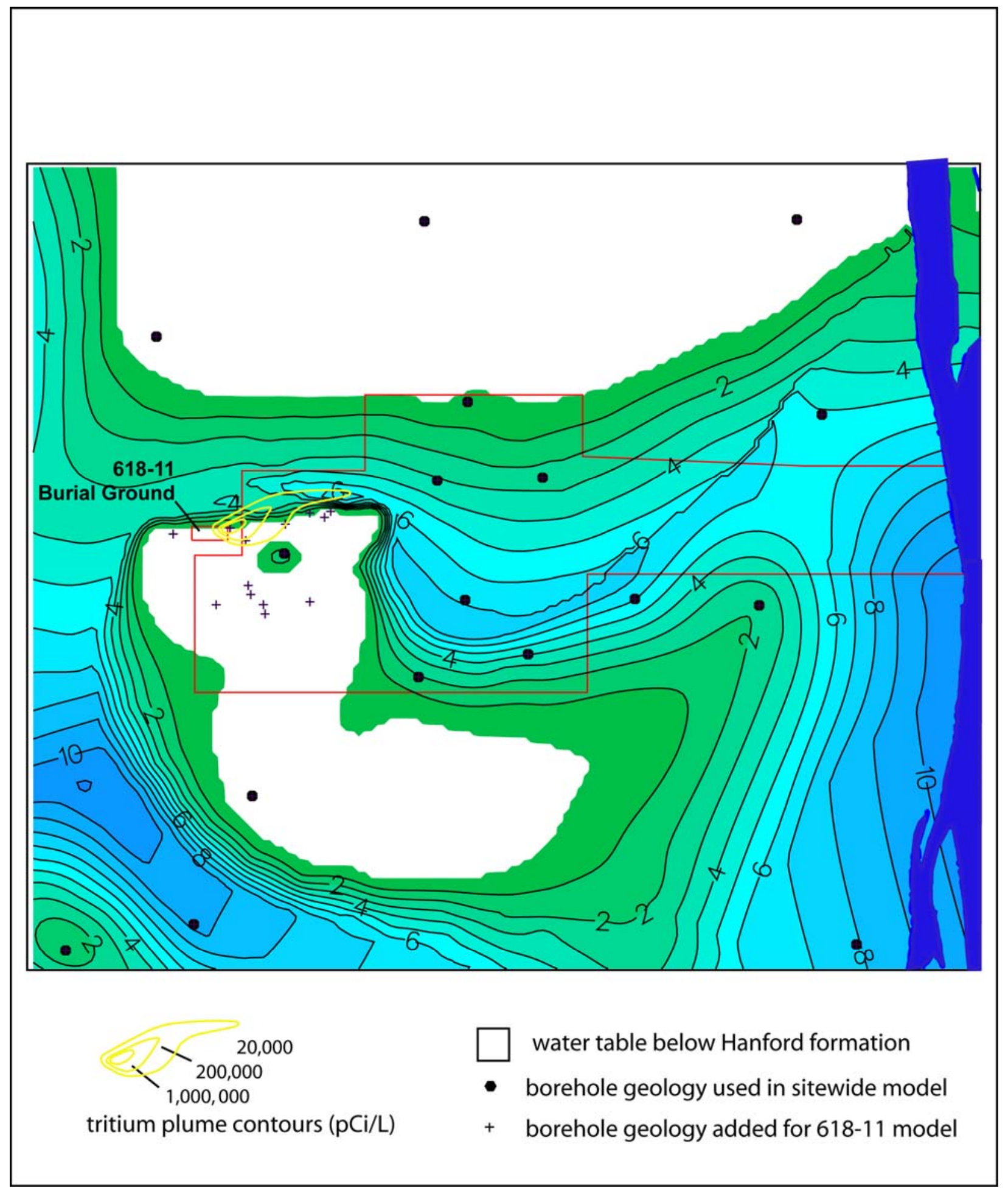

Figure 4.4. Hanford Formation Saturated Thickness (m) Based on the 2001 Water Table 
Hydraulic property data for the Hanford Site unconfined aquifer have been derived mainly from aquifer pumping tests and, in a few cases, from laboratory permeameter tests. These results have been documented in dozens of published and unpublished reports over the past 50 years. A summary of available data for the unconfined aquifer was provided in DOE (1988) and an updated summary was provided in Thorne and Newcomer (1992) together with an evaluation of selected pumping test analyses.

Hydraulic property measurements from aquifer pumping tests are not available from the wells in the immediate vicinity of the 618-11 burial ground. Across the Hanford Site, aquifer tests indicate that the $\mathrm{K}_{\mathrm{h}}$ of the Hanford formation varies between approximately 1 to 10,000 meters (3.28 to 32,808 feet) per day (Thorne and Newcomer 1992; DOE 1988). However, the maximum hydraulic conductivity that can be measured by an aquifer test is limited by the well efficiency and flow rate that can be pumped with available equipment. As a result, the upper limit of $\mathrm{K}_{\mathrm{h}}$ for coarse gravel flood deposits of the Hanford formation is probably greater than the values interpreted from existing field tests. The ratio of $\mathrm{K}_{\mathrm{h}}$ to $\mathrm{K}_{\mathrm{v}}$ (vertical anisotropy) for the Hanford formation has been measured in a few multiple well pumping tests at values of 0.2 to 0.5 , but it may approach 1.0 in relatively clean gravel zones where stratified layers of finer-grained material are not present. Ringold gravel units consist of sand to muddy sandy gravel with varying degrees of consolidation and/or cementation. Measured $K_{h}$ values of these units vary within the range of about 0.1 to 200 meters ( 0.32 to 656 feet) per day. The average $K_{h}$ value measured from aquifer pumping tests in the Ringold gravel is about 20 meters (65.6 feet) per day. However, the geometric mean is about 7.5 meters (24.6 feet) per day. A few multiple well aquifer tests suggest vertical anisotropy is in the range of 0.01 to 0.1 . Therefore, the range of $K_{v}$ is estimated at about 0.001 to 20 meters $(0.003$ to 65.6 feet) per day.

Storativity and specific yield can be calculated from multiple-well aquifer tests. Specific yield values from these tests ranged from 0.11 to 0.2 for the Ringold Formation gravels and from 0.2 to 0.37 for the Hanford formation. The average specific yield was 0.15 for the Ringold gravels and 0.27 for the Hanford formation. The only test conducted in the coarse grained facies of the Cold Creek unit resulted in a specific yield of 0.15 . However, some of the test results are highly uncertain because of the effects of non-ideal test conditions, such as partially penetrating wells and aquifer heterogeneity. Such conditions generally have a more significant effect on the determination of storage properties than on the determination of transmissivity. Moench (1994) demonstrated that these conditions can affect specific yield values calculated from type-curve analysis of aquifer pumping tests, and usually result in the calculated values being low.

\subsection{8-11 Tritium Plume Conceptual Model}

The 618-11 burial ground received waste between March 1962 and December 1967 (Demiter and Greenhalgh 1997). The site consists of three trenches, two to five large-diameter caissons, and fifty vertical pipe storage units. The site covers an area of 3.5 hectares (8.6 acres) and is located approximately 300 meters (1,000 feet) west of Energy Northwest Plant 2 (WNP-2). The trenches are 274.3 meters (900 feet) long by 15.2 meters (50 feet) wide. The vertical pipe units are five 208-liter (55-gallon) drums welded together end-to-end and are approximately 4.6 meters (15 feet) long by 55.9 centimeters (22 inches) in diameter. The caissons are 2.4-meter- (8-foot-) diameter metal pipe, 3 meters (10 feet) long, buried vertically 4.6 meters ( 15 feet) below grade, connected to the surface by 
offset 91.4-centimeter- (36-inch-) diameter pipe with a dome-type cap. All vertical pipe units and caissons were capped with concrete and covered with native sediment as they were filled. The bottoms of each vertical pipe unit and caisson are open.

Waste was sent to the 618-11 burial ground from the 324, 325 and 327 Building hot cells and the Plutonium Recycle Test Reactor in the 300 Area. Inventories of the waste do not specifically mention that tritium was disposed to the burial ground, though hydrogen gas (a possible misnomer) was identified. Shortly after the site was closed, it was covered with 1.2 meters (4 feet) of soil. In 1983, the surface of the site was stabilized with an additional 0.6 meter ( 2 feet) of clean material and planted with wheatgrass. The bottoms of the trenches and caissons are estimated to be approximately 9.1 meters (30 feet) below grade, while the bottoms of the vertical pipe units are estimated to be 6.4 meters ( 21 feet) below grade. The site perimeter is fenced and marked with concrete markers. Plants in the area show no obvious signs of vegetative stress that would indicate radiological or chemical constituent uptake from either the waste site or the unplanned releases that have occurred at the site.

The mechanisms controlling tritium release from the 618-11 burial ground are not well understood or have not been well characterized and thus, development of a detailed conceptual model of historic release(s) from the site is not possible. There are two components of contaminant transport between the 618-11 waste forms and the water table including (1) release of contaminants from containment structures (i.e., vertical storage pipes and caissons) and (2) migration of contamination through the vadose zone to the water table. Factors controlling transport for the first component include waste form and container integrity, condition of the containment structures, and transport mechanisms within the unsaturated zone. The primary driver controlling transport of contaminants through the vadose zone and into the unconfined aquifer is surface recharge. Although surface recharge on the Hanford Site is relatively low, with recharge estimates in the vicinity of the 618-11 burial ground on the low end of the estimate range of values for the site (5 to 10 millimeters [0.2 to 0.4 inch] per year; Fayer and Walters 1995), episodic occurrences of elevated recharge may provide a means for increase infiltration through the vadose zone and pulsed release of tritium to groundwater. Examples of increased recharge events include application of water during establishment of wheatgrass groundcover at the site following the 1983 stabilization effort and atypical winter weather conditions resulting in significant quantities of snow cover, and in some cases rain of snow events, that significantly increase short-term natural recharge rates.

The 618-11 tritium plume was first identified in January 1999 and its nature and extent were further characterized during subsequent field investigations (Dresel et al. 2000) and letter reports. ${ }^{2,3}$ Characterization and monitoring data collected through the fourth quarter of FY 2001 form the basis for the conceptual model of initial tritium distribution at the site and are being used as initial conditions in a numerical modeling evaluation of the fate and transport of tritium contaminated groundwater from the 618-11 burial ground (Figure 4.5). It should be noted that groundwater contamination from the 200 East Area extends

\footnotetext{
${ }^{2}$ Letter report from Bechtel Hanford, Inc. to U.S. Department of Energy, Tritium Groundwater Investigation at the 618-11 Burial Ground, September 2001, dated September 28, 2001. Prepared by JV Borghese, WJ McMahon, and RW Ovink (CH2M HILL Hanford, Inc.) for Bechtel Hanford, Inc., Richland, Washington.

${ }^{3}$ Letter Report from PE Dresel and MP Bergeron (Pacific Northwest National Laboratory) to KM Thompson and MJ Furman (U.S. Department of Energy, Richland, Washington), Evaluation of the Impact of Tritium Contamination in Groundwater from the 618-11 Burial Ground at the Hanford Site, dated December 20, 2001.
} 


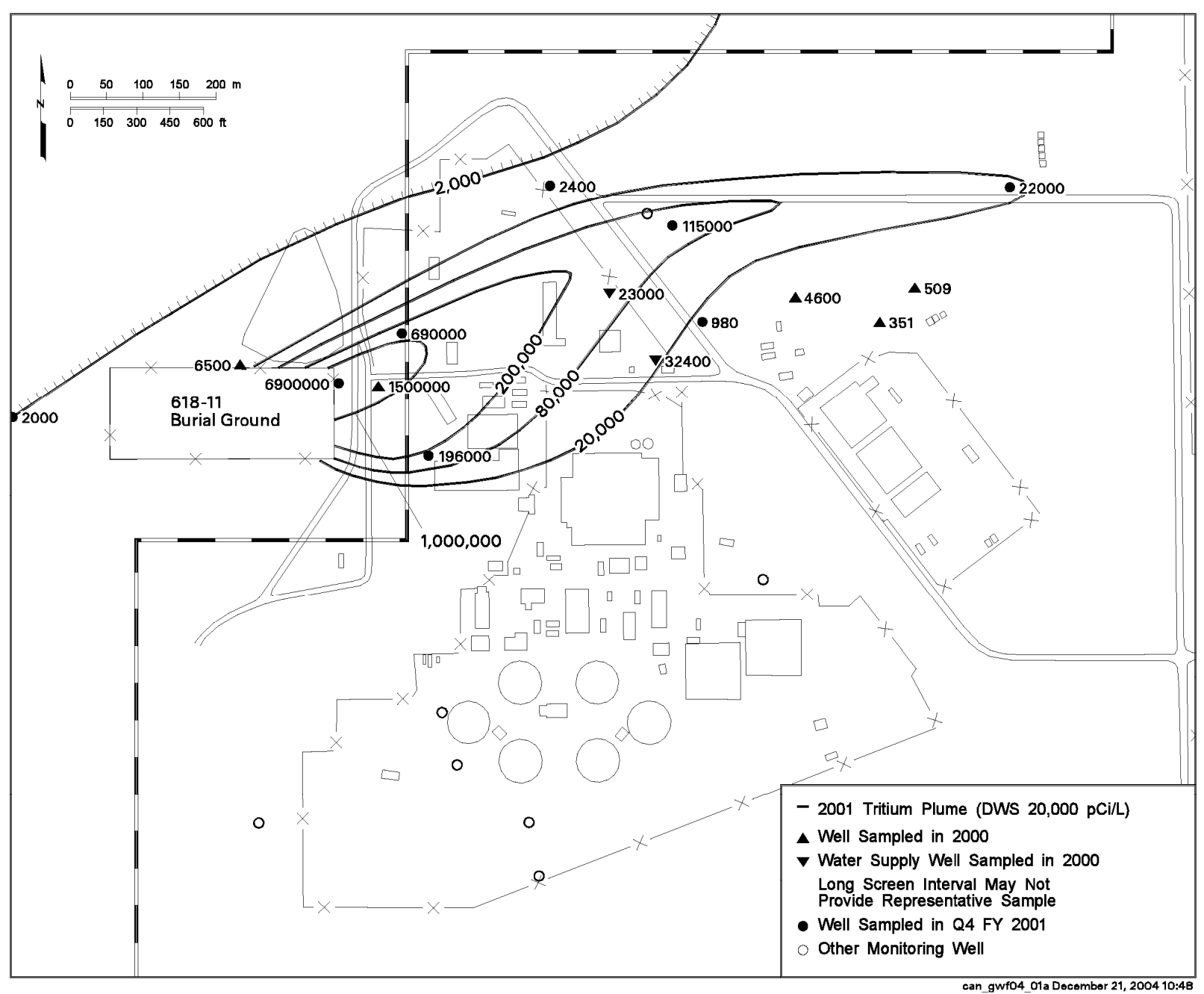

Figure 4.5. Tritium Plume Downgradient of 618-11 Burial Ground, 2001 Conditions

over a large portion of the Hanford Site to areas adjacent to the 618-11 burial ground. However, an area of anomalously low tritium has been noted east of the burial ground when compared to the surrounding plume from the 200 East Area. This local area tritium distribution, which simplifies delineation of the higher concentration tritium plume associated with the burial ground, is thought to be the result of hydrogeologic controls that effect groundwater flow in the area.

As discussed previously, the mechanisms controlling tritium release from the 618-11 burial ground are not well understood or have not been well characterized; therefore, development of a detailed conceptual model of historic release(s) from the site is not possible. Due to these limitations, a release history from the burial ground was not incorporated into the numerical model. Instead, initial conditions were developed based on the distribution of tritium in the groundwater in 2001. The tritium distribution was developed from measured concentrations in wells and knowledge of the geologic structure of the aquifer.

Beneath the 618-11 burial ground, the water table is in the Ringold Formation, and the Hanford formation is completely above the water table. However, the tritium plume moves into saturated Hanford 
formation and Cold Creek unit gravels immediately downgradient of the burial site (Figure 4.4). As the tritium migrates northward into the area with saturated Hanford formation gravels, the plume appears to move eastward at a higher rate than observed within Ringold formation sediment. Given the difference in hydraulic properties between these two units, this type of response would be expected.

Only limited information is available on the vertical distribution of tritium contamination in the vicinity of the 618-11 burial ground. Borghese et al. ${ }^{4}$ collected samples at discrete depths in borehole C3254, completed as monitoring well 699-13-2D (Figure 4.1) and analyzed for tritium. Results are shown in Table 4.1. The higher concentration of tritium deeper in the aquifer is thought to be a result of downward movement of groundwater in response to pumping during construction of the Washington Public Power Supply System (WPPSS-1) power plant. ${ }^{4}$ Based on this information, tritium concentration in the Ringold formation was assumed to be twice as high from 5 to 10 meters (16.4 to 32.8 feet) below the water table as it is in the upper 5 meters ( 16.4 feet) of the aquifer. The concentration from 10 to 15 meters ( 32.8 to 49.2 feet) below the water table was assumed to be the same as in the upper 5 meters (16.4 feet). Concentration was assumed to be 0 more than 15 meters (49.2 feet) below the water table. This distribution was only applied for the Ringold gravel portion of the aquifer. In the saturated portion of the Hanford formation and Cold Creek unit, the tritium distribution was assumed to be constant with depth over the upper 15 meters (49.2 feet) of the aquifer. A three-dimensional view of the initial condition plume implemented in the 618-11 model is shown in Figure 4.6.

Table 4.1. Tritium Measured at Discrete Depths in Borehole C3254

\begin{tabular}{|c|c||}
\hline $\begin{array}{c}\text { Depth Below Water Table } \\
(\mathrm{m})\end{array}$ & Tritium (pCi/L) \\
\hline 0.1 & 690,000 \\
\hline \hline 2.7 & 634,000 \\
\hline 5.7 & $1,330,000$ \\
\hline 11.4 & $1,300,000$ \\
\hline 12.9 & 503,000 \\
\hline
\end{tabular}

\subsection{8-11 Tritium Plume Trends}

The general shape of the tritium plume has remained nearly constant since the first maps were drawn in 2000. A comparison of conditions in 2001 and 2004 are provided in Figures 4.5 and 4.7, respectively. Both groundwater concentration data and mapped geologic controls (i.e., the Hanford/Ringold contact) were considered during development of these tritium plume maps. Tritium concentrations near the 618-11 burial ground (well 699-13-3A) show a decreasing trend since the occurrence of peak values in 2000 (Figure 4.8; see Figure 1.3 for well location map). Current levels ( 2.3M pCi/L) still greatly exceed drinking water standards. The decrease in concentration observed close to the source cannot be entirely encountered for by radioactive decay indicating that transport processes are impacting tritium concentrations and suggesting dispersal of a "pulse" release that was first observed in 1999; no tritium

\footnotetext{
${ }^{4}$ Letter report from Bechtel Hanford, Inc., to U.S. Department of Energy, Tritium Groundwater Investigation at the 618-11 Burial Ground, September 2001, dated September 28, 2001. Prepared by JV Borghese, WJ McMahon, and RW Ovink (CH2M HILL Hanford, Inc.) for Bechtel Hanford, Inc., Richland, Washington.
} 
data was available immediately downgradient of the burial ground prior to this date. Relatively constant or gradually increasing trends are observed at wells along the downgradient flow path from the burial ground (e.g., wells 699-13-1E and 699-13-0A), indicating a relatively slow downgradient migration of the tritium plume (Figures 4.9 and 4.10, respectively).

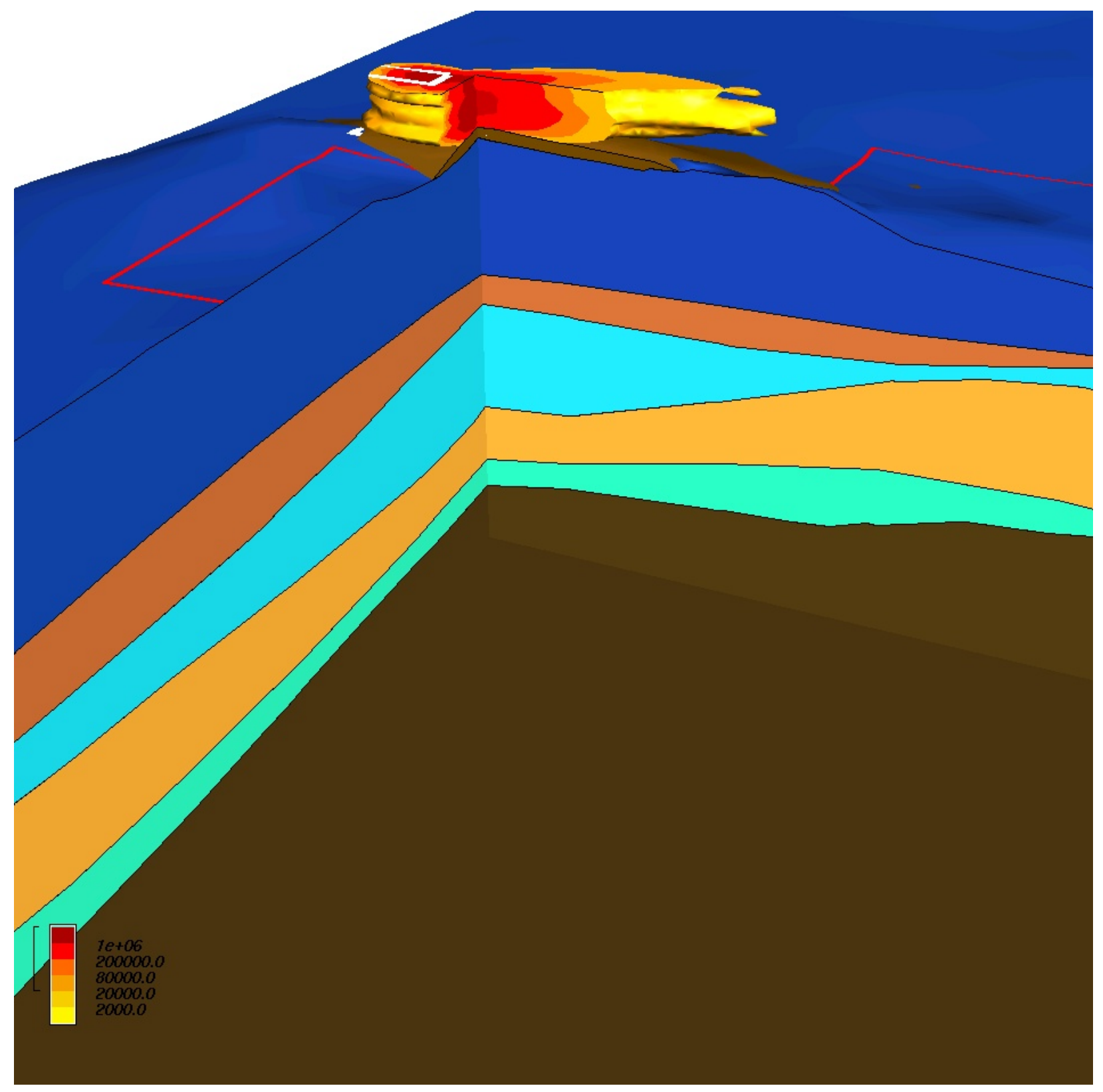

Figure 4.6. Initial Condition Tritium Plume Implemented for the 618-11 Model Compared to Hydrogeologic Layering 


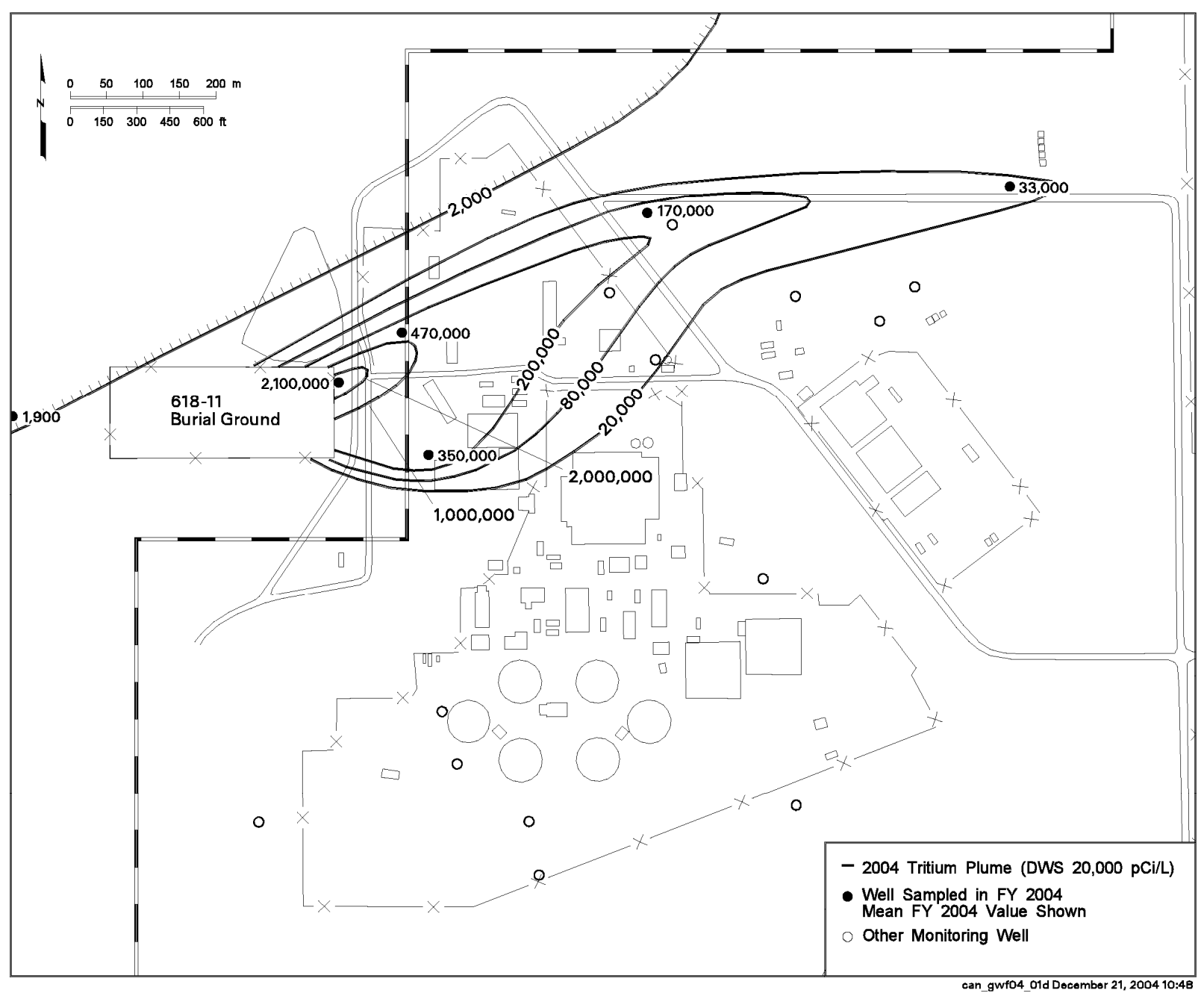

Figure 4.7. Tritium Plume Downgradient of 618-11 Burial Ground, 2004 Conditions 


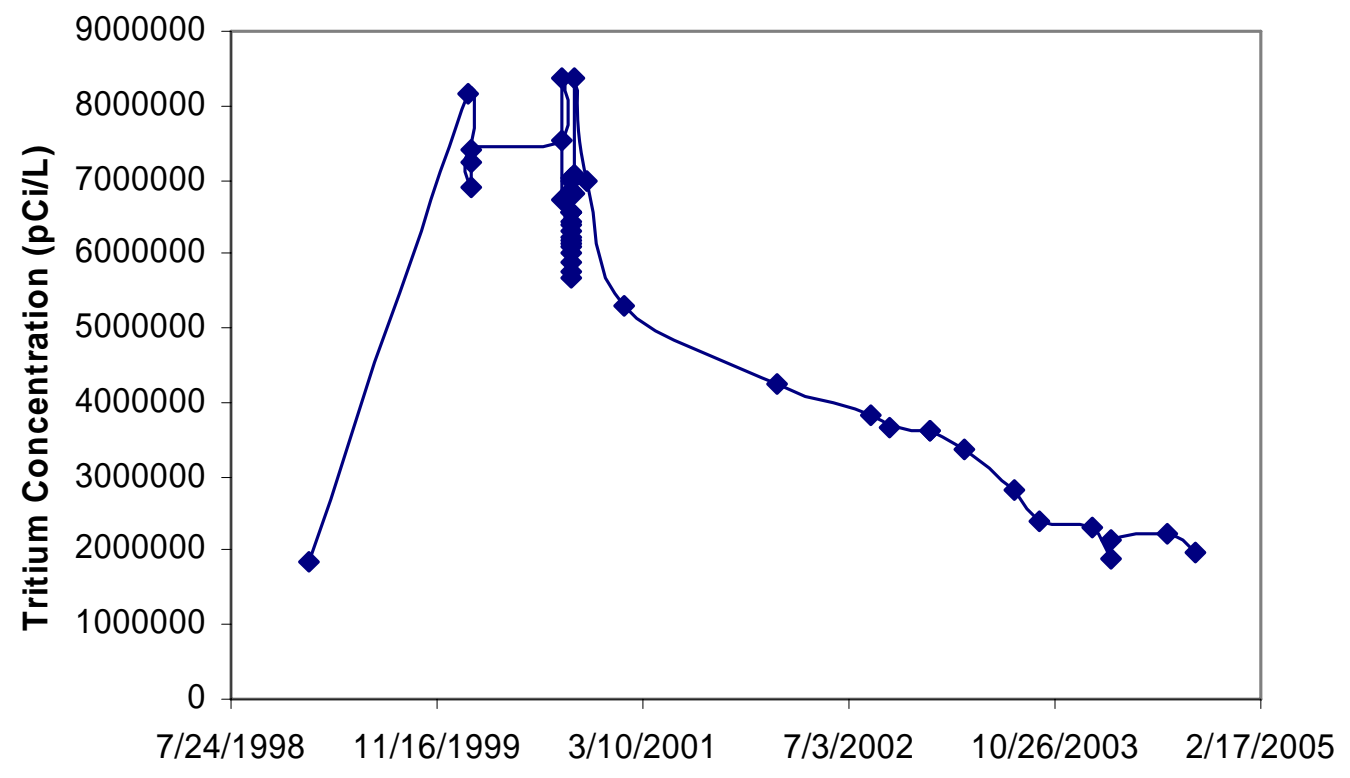

Figure 4.8. Tritium Concentration Trend Plot for Monitoring Well 699-13-3A

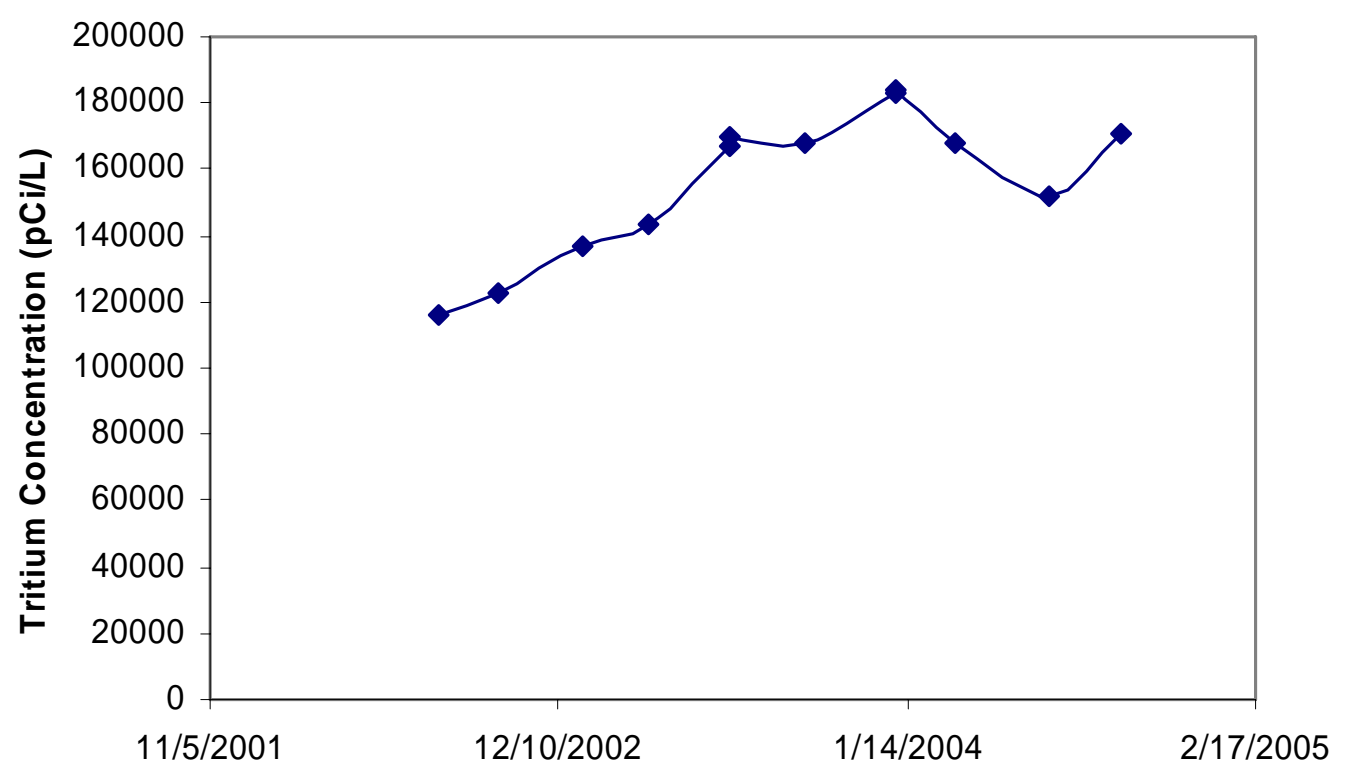

Figure 4.9. Tritium Concentration Trend Plot for Monitoring Well 699-13-1E 


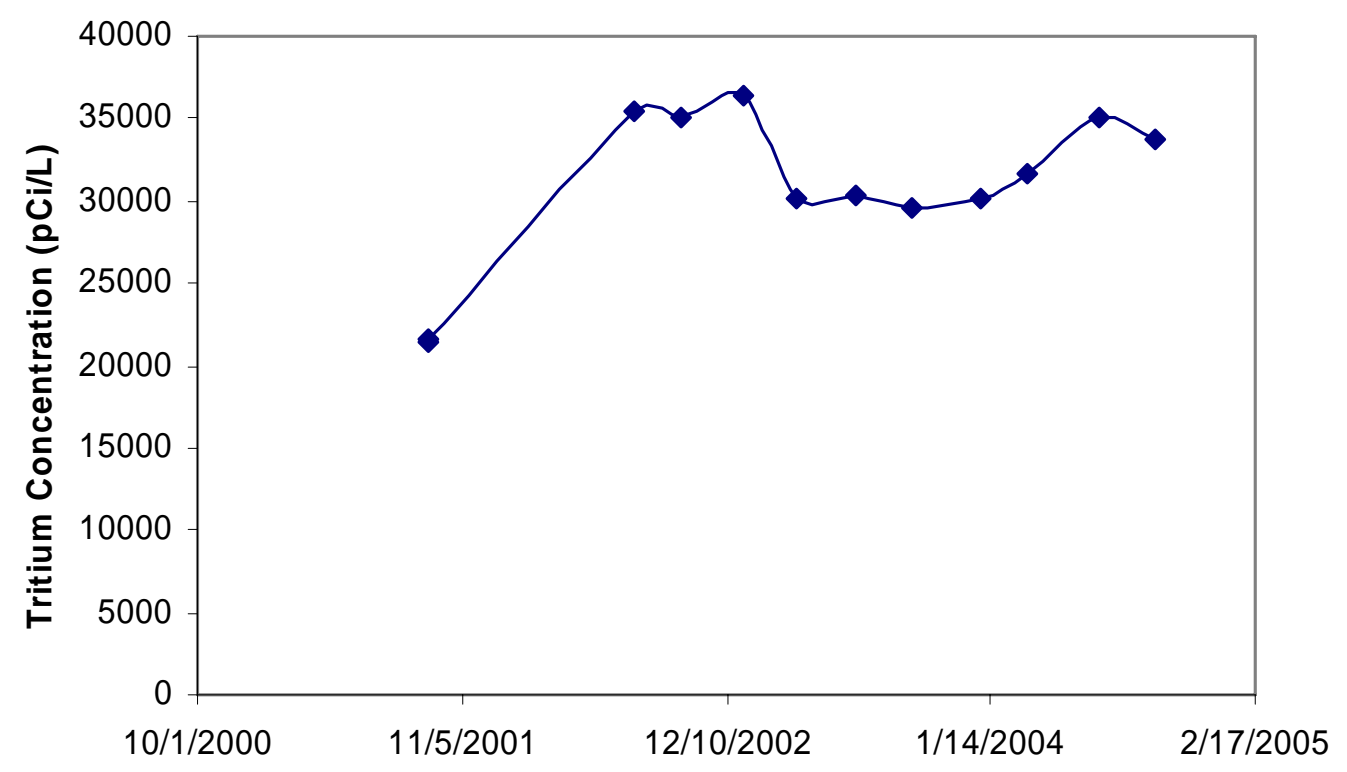

Figure 4.10. Tritium Concentration Trend Plot for Monitoring Well 699-13-0A

\subsection{Groundwater Flow and Transport Modeling}

The local scale 618-11 burial ground model is a refinement of the regional scale Hanford groundwater model (Vermeul et al. 2003). The regional grid covering the local scale model region was extracted and used as the starting point for the refinement process. Two sequential, subgrid refinements of the grid were performed. The inner-most refinement encompasses the initial extent of the 618-11 burial ground tritium plume. Figure 4.11 shows the refined, local scale grid that was used in this modeling effort. The grid spacing of the regional groundwater model corresponds to the large square grids that measure 250 meters (820 feet) on a side. The region of highest refinement has grid spacing of 28 meters (92 feet). The complete model consists of 4759 surface nodes and 4702 elements. The subsurface domain was divided into 27 layers, with the upper regions represented by 5-meter (16.4-foot) thick sublayers.

Boundary conditions were developed to approximate groundwater flow conditions through and downgradient of the site over the simulation period (2001 through 2076). Average Columbia River stage was used to define the east held-head boundary condition that remained fixed throughout the simulation period. During development of the local scale model, the north and south model boundaries were located such that, based on available water-level contour maps, groundwater flow was parallel to the model boundaries. This placement allowed for the north and south boundaries of the model to be represented by a no-flow boundary condition. The west boundary of the model was set as held head, based on observed heads in 2001 at the start of the simulation period. Although a declining head boundary condition based on predicted water-level decline from the System Assessment Capability regional groundwater flow model was developed for this modeling effort, it was not used during this initial modeling effort. Subsequent model runs will incorporate this declining head boundary condition. Although the declining head boundary condition was not implemented in time for inclusion in this report, the constant head solution provides a conservative estimate of tritium transport at the site since the declining head 
boundary will act to decrease tritium transport by (1) lowering the gradient through the burial ground and (2) decreasing the thickness and areal extent of saturated Hanford and Cold Creek gravels (i.e., the higher permeability formations).

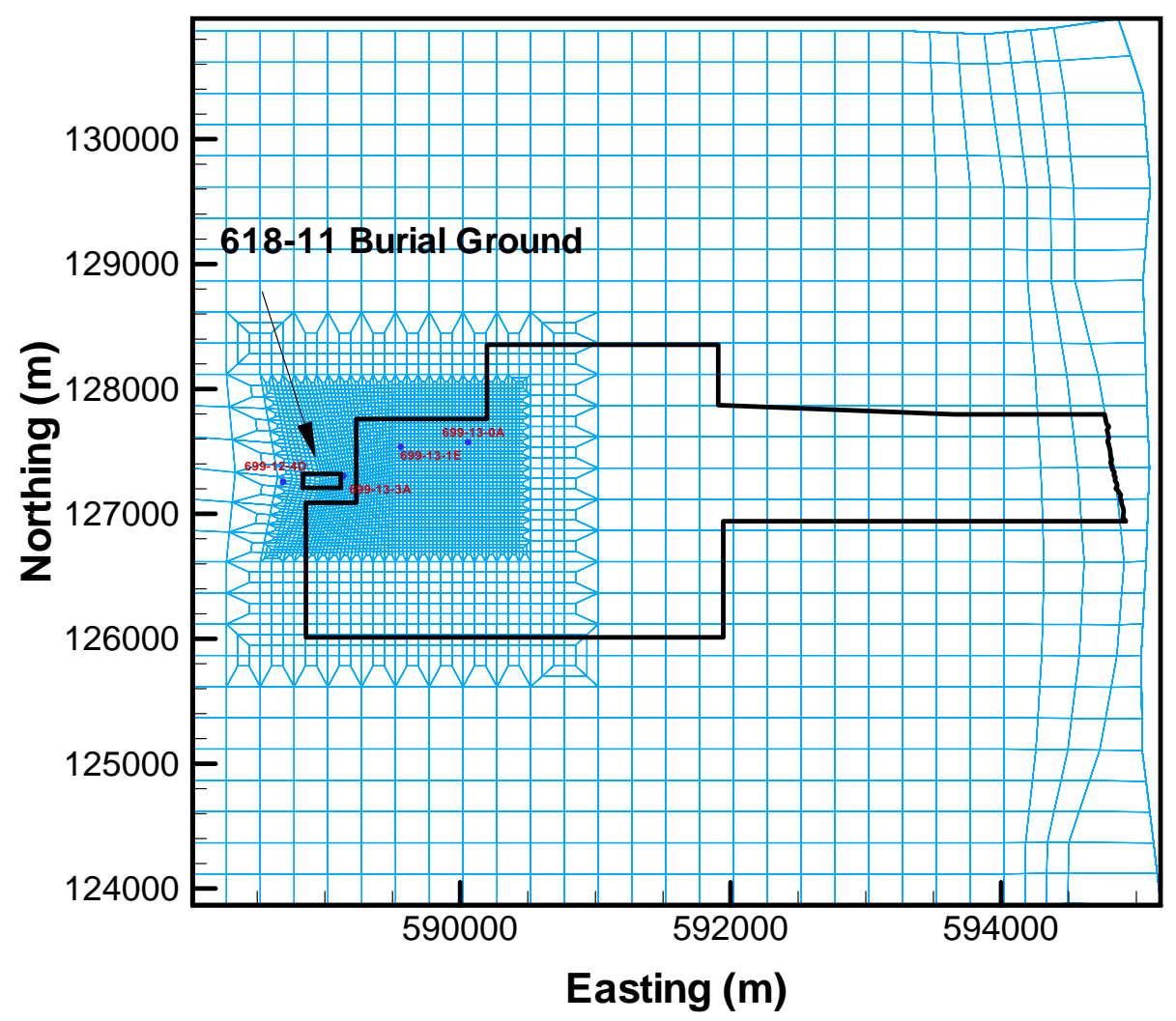

Figure 4.11. 618-11 Burial Ground Model Grid

Preliminary tritium transport simulations indicate that mechanisms associated with dilution, dispersion, and radioactive decay have in the past, and will continue, to attenuate the tritium plume at the 618-11 burial ground and limit the risk associated with exposure to the primary receptors (i.e., the Columbia River and Energy Northwest water supply wells). Prior to running forward predictive simulations over the full period of interest, model predictions at several locations downgradient of the burial ground were compared with observed concentrations to evaluate model fit. This process was conducted in an iterative fashion, with the distribution of tritium residuals (i.e., the difference between observed and simulated values) guiding the development of alternate conceptual models that would address unacceptably high residual values and ultimately provide a reasonable fit to the observed tritium concentration trend data. Results from the developed model are provided below.

A comparison of simulated and observed tritium concentration at two downgradient monitoring well locations is provided in Figures 4.12 and 4.13. As indicated, the model provides a reasonable representation of the tritium concentration trend both immediately downgradient of the site (well 699-13-3A) and near the leading edge of the plume (well 699-13-0A). This relatively good match increases confidence in both the site conceptual model and predictive simulations of tritium fate and transport. 


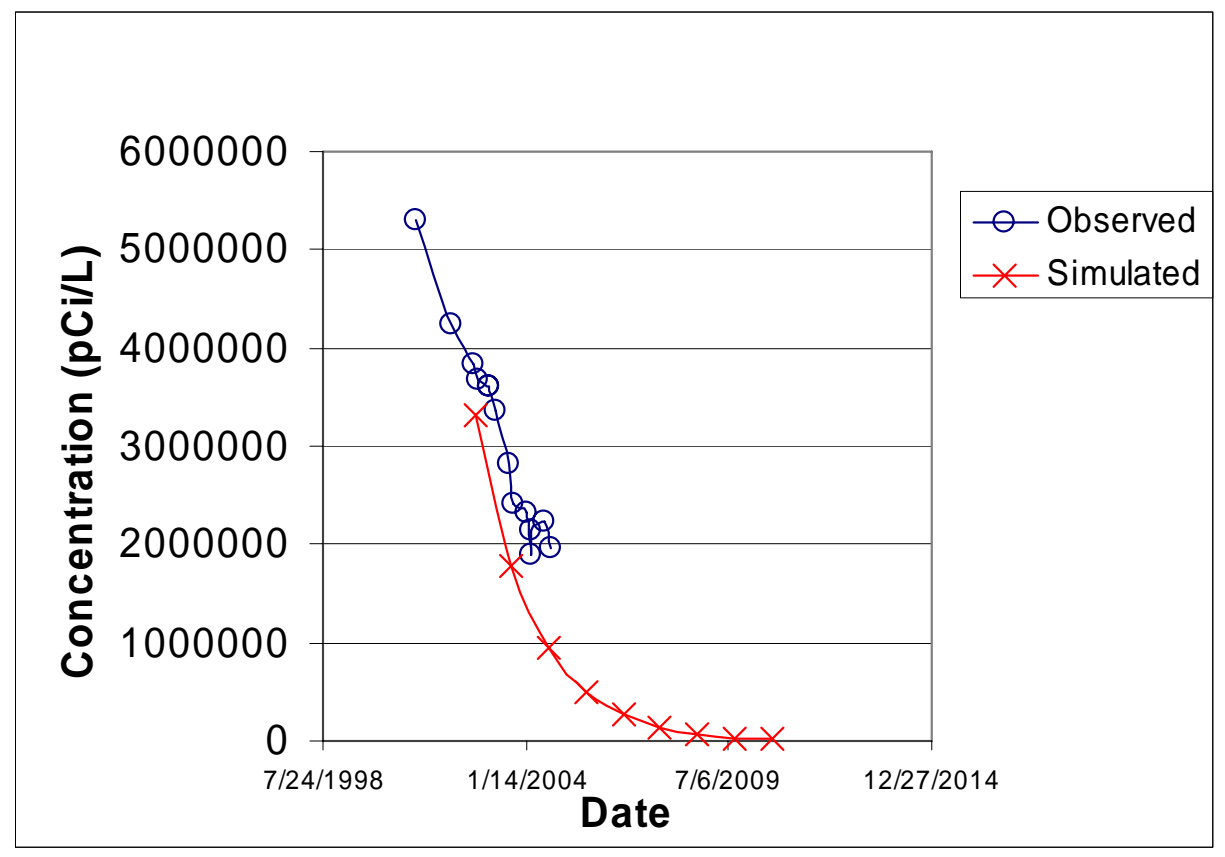

Figure 4.12. Comparison of Simulated and Observed Tritium Concentration at Well 699-13-3A

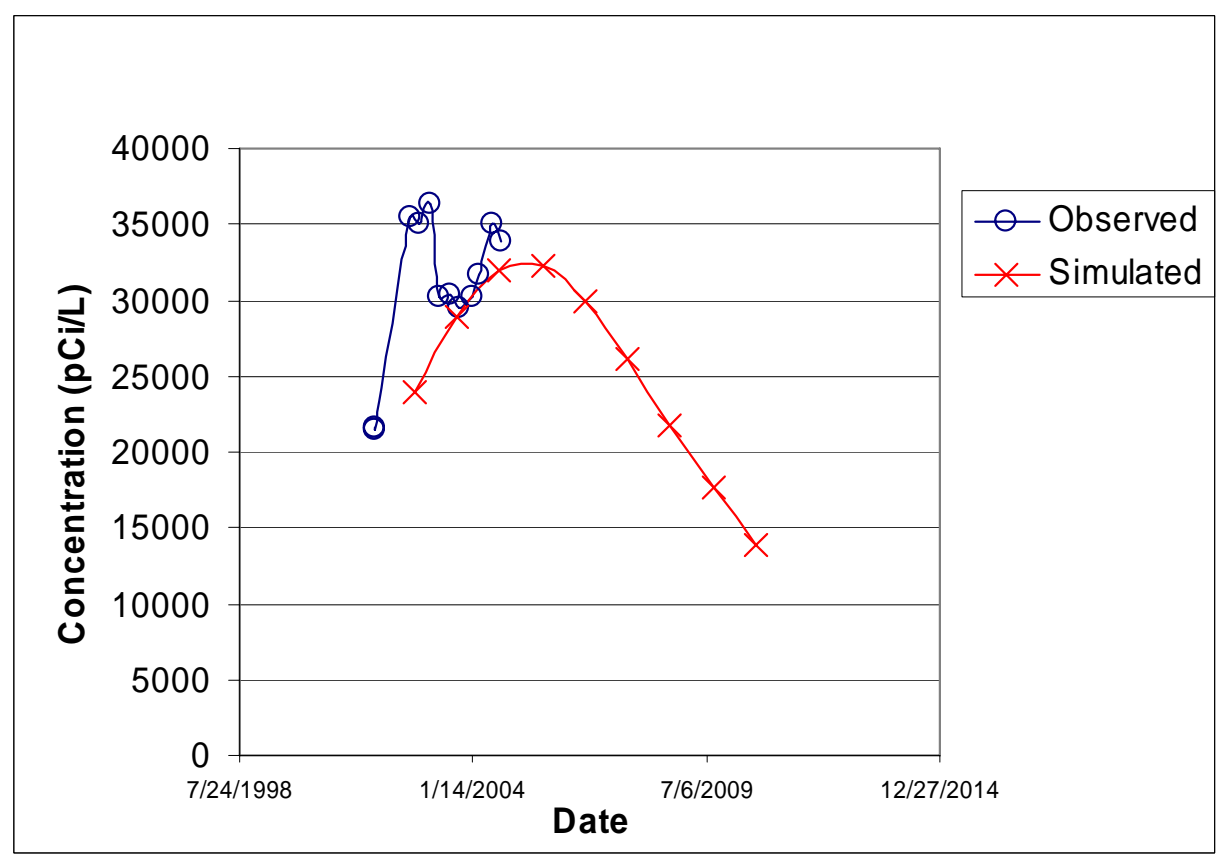

Figure 4.13. Comparison of Simulated and Observed Tritium Concentration at Well 699-13-0A

A plot of maximum concentration with time over the full model domain is shown in Figure 4.14. As indicated, the maximum tritium concentration declines to below the drinking water standard of 20,000 pCi/L by 2024. Tritium concentration with time at the two primary receptor locations (i.e., the Columbia River and Energy Northwest water supply wells, MW-31 and -32) is provided in Figures 4.15 and 4.16, respectively. Tritium from the 618-11 burial ground is not expected to migrate to either of these 
locations at high enough concentrations to pose a significant risk. These simulations do not consider pumping from the Energy Northwest water supply wells that, if sustained at high rates for long periods of time, could increase the observed concentration at this location. Figures 4.17 through 4.20 provide a time series sequence of tritium concentration contour plots starting in 2001 and extending through 2027 when maximum tritium concentrations within the plume have decreased to below drinking water standards.
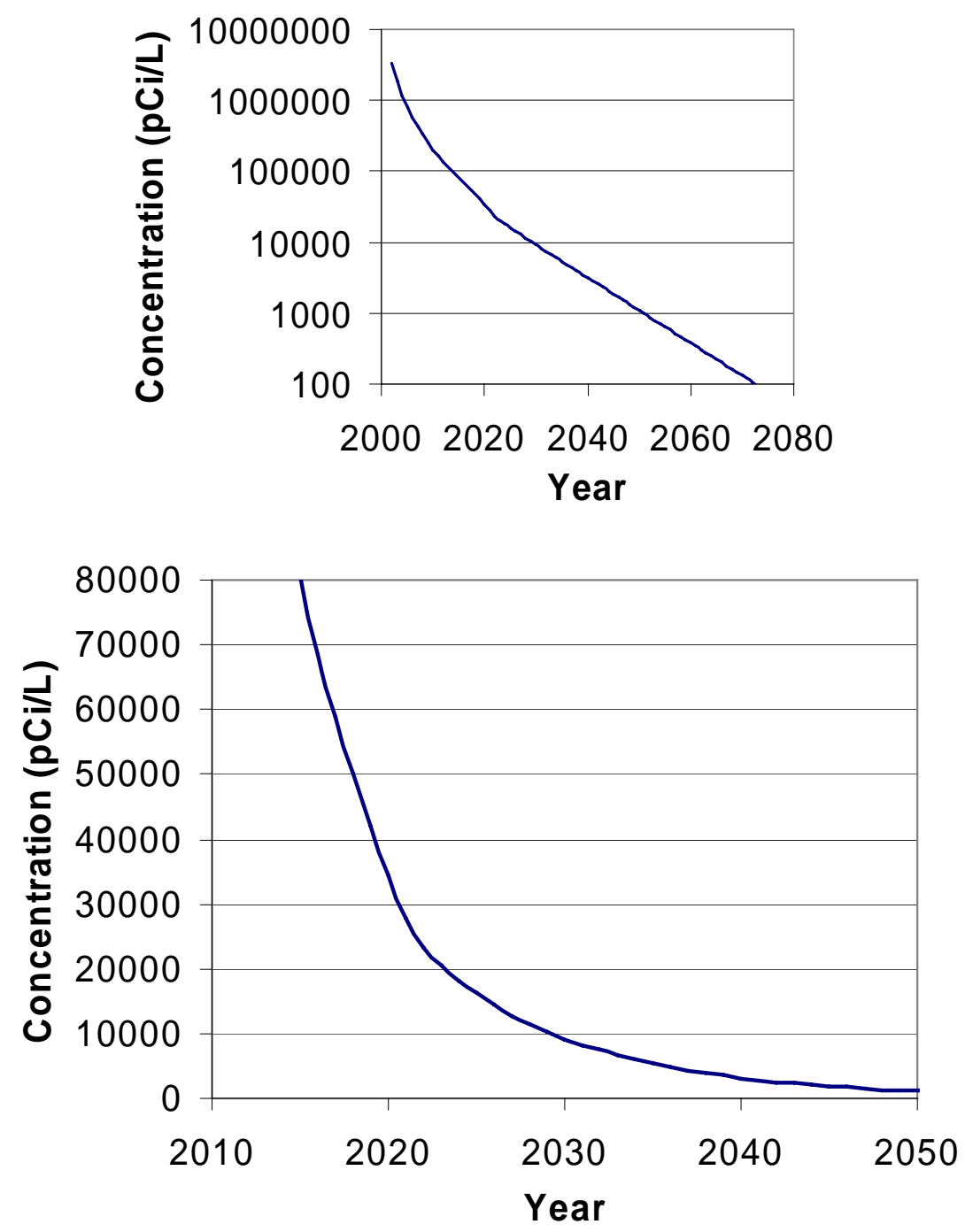

Figure 4.14. Maximum Predicted Concentration throughout the 618-11 Tritium Plume 


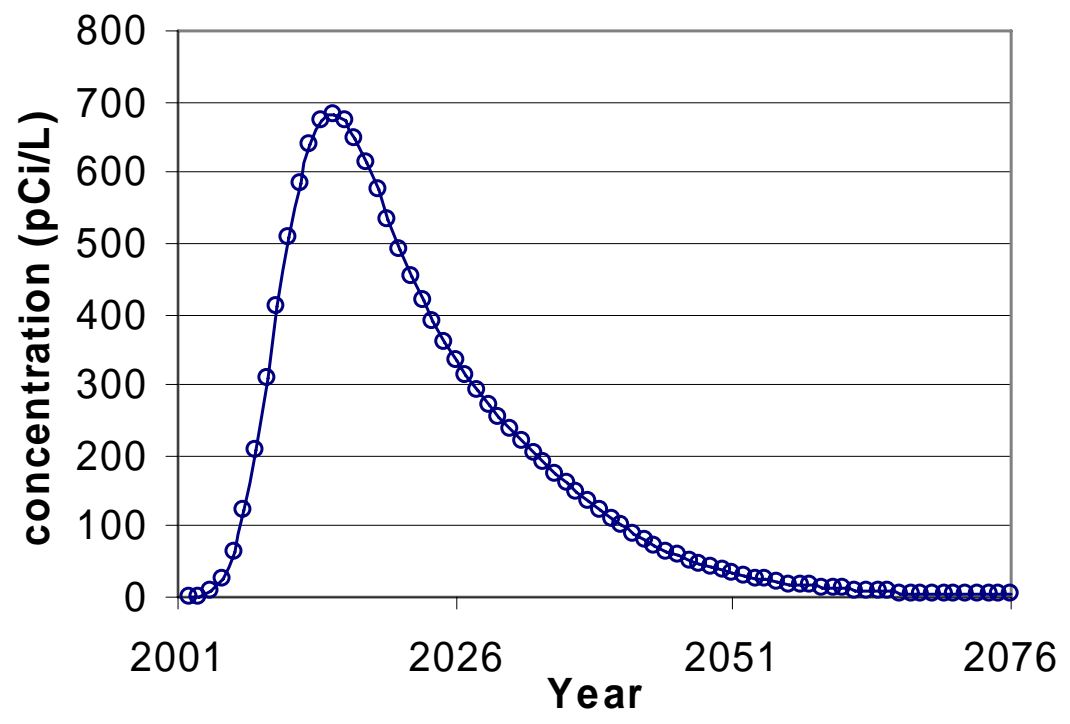

Figure 4.15. Predicted Tritium Concentration at the Columbia River Boundary

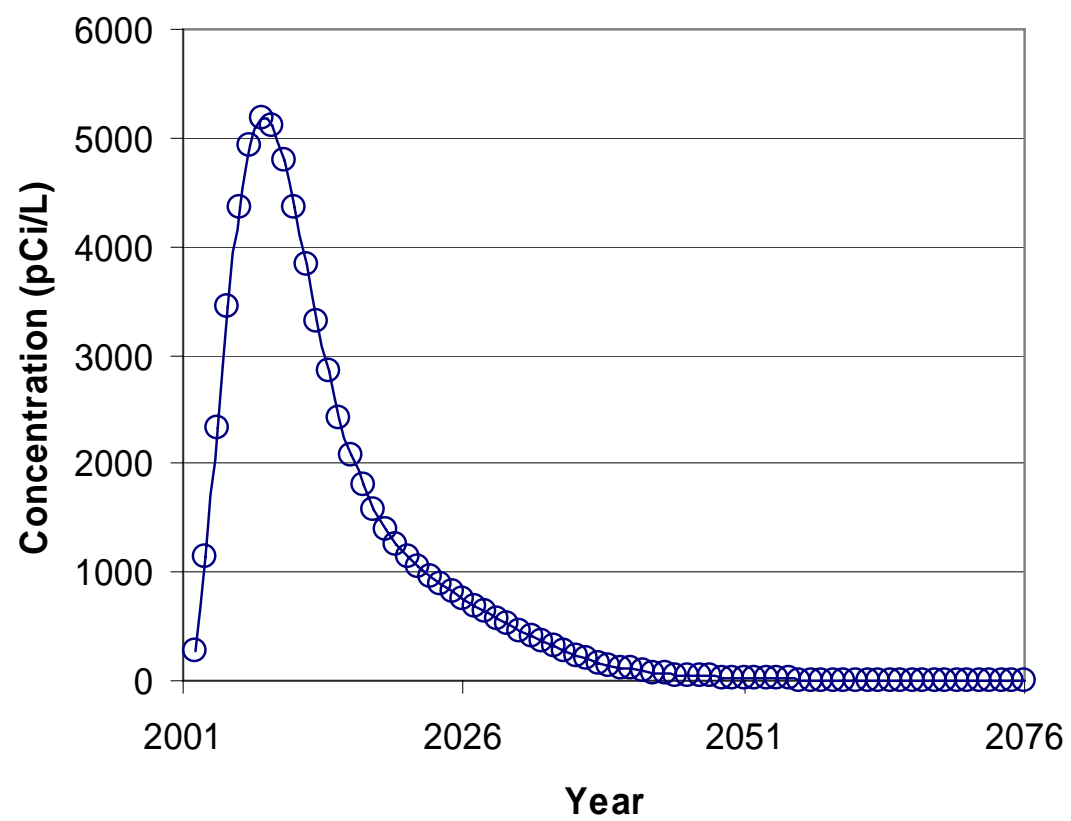

Figure 4.16. Predicted Tritium Concentration at the Energy Northwest Water Supply Wells (MW-31 and MW-32) 


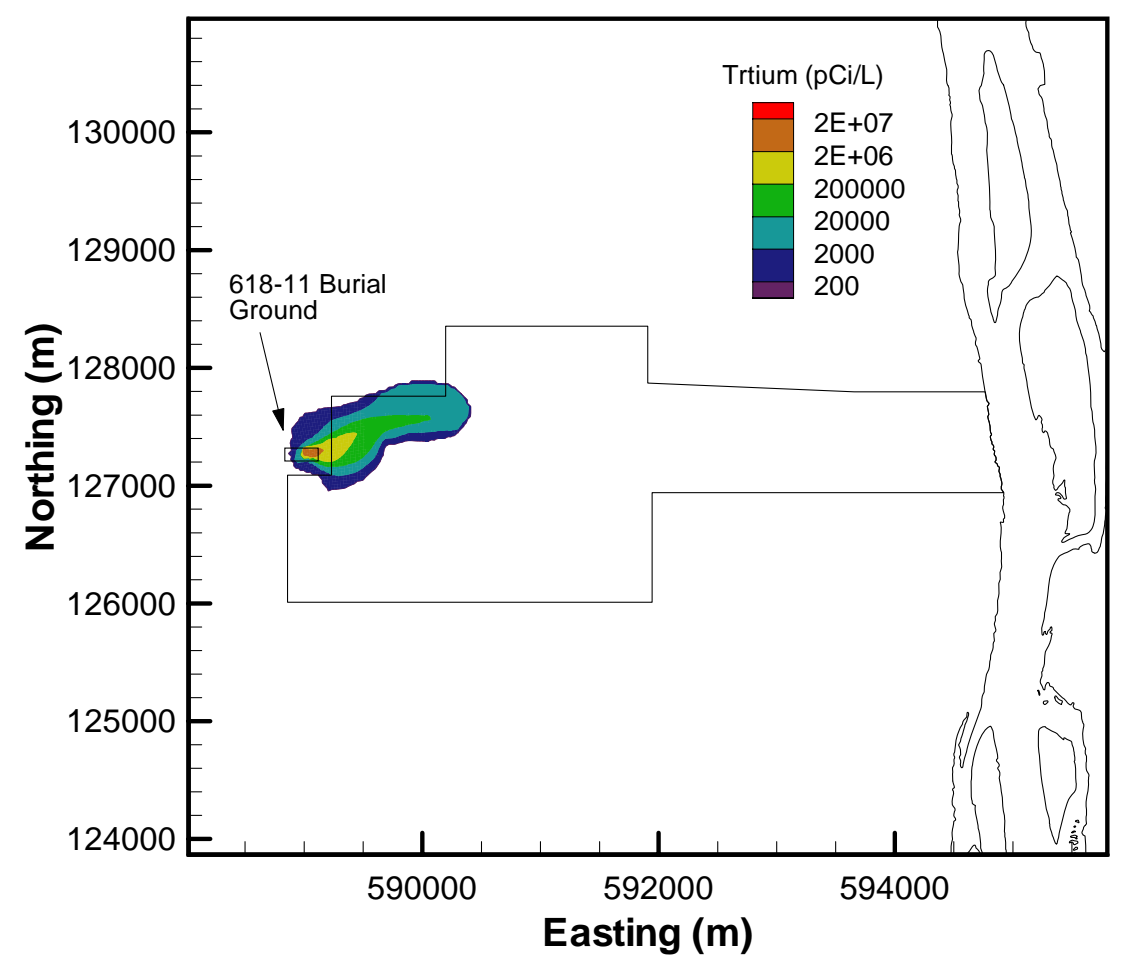

Figure 4.17. Tritium Plume at the 618-11 Burial Ground, Initial Conditions in 2001

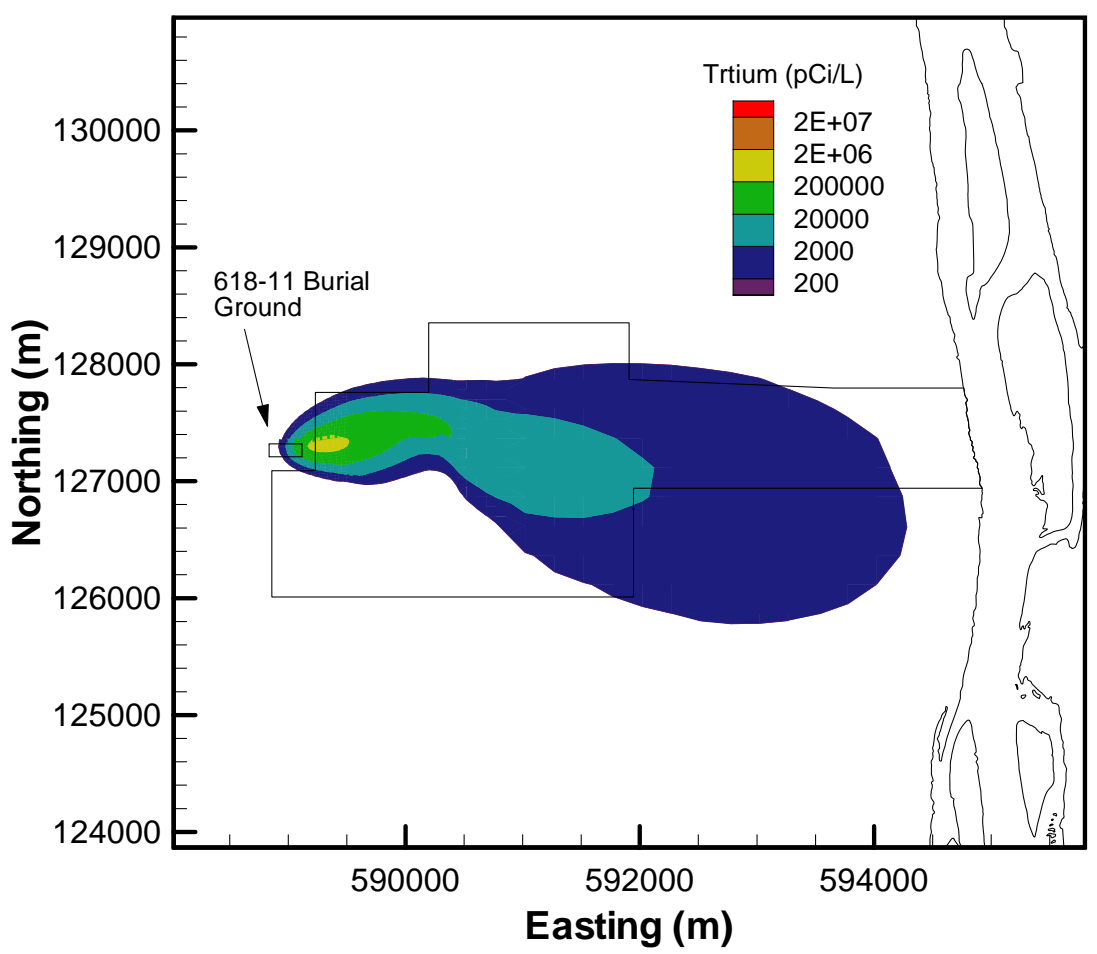

Figure 4.18. Tritium Plume at the 618-11 Burial Ground in 2007 


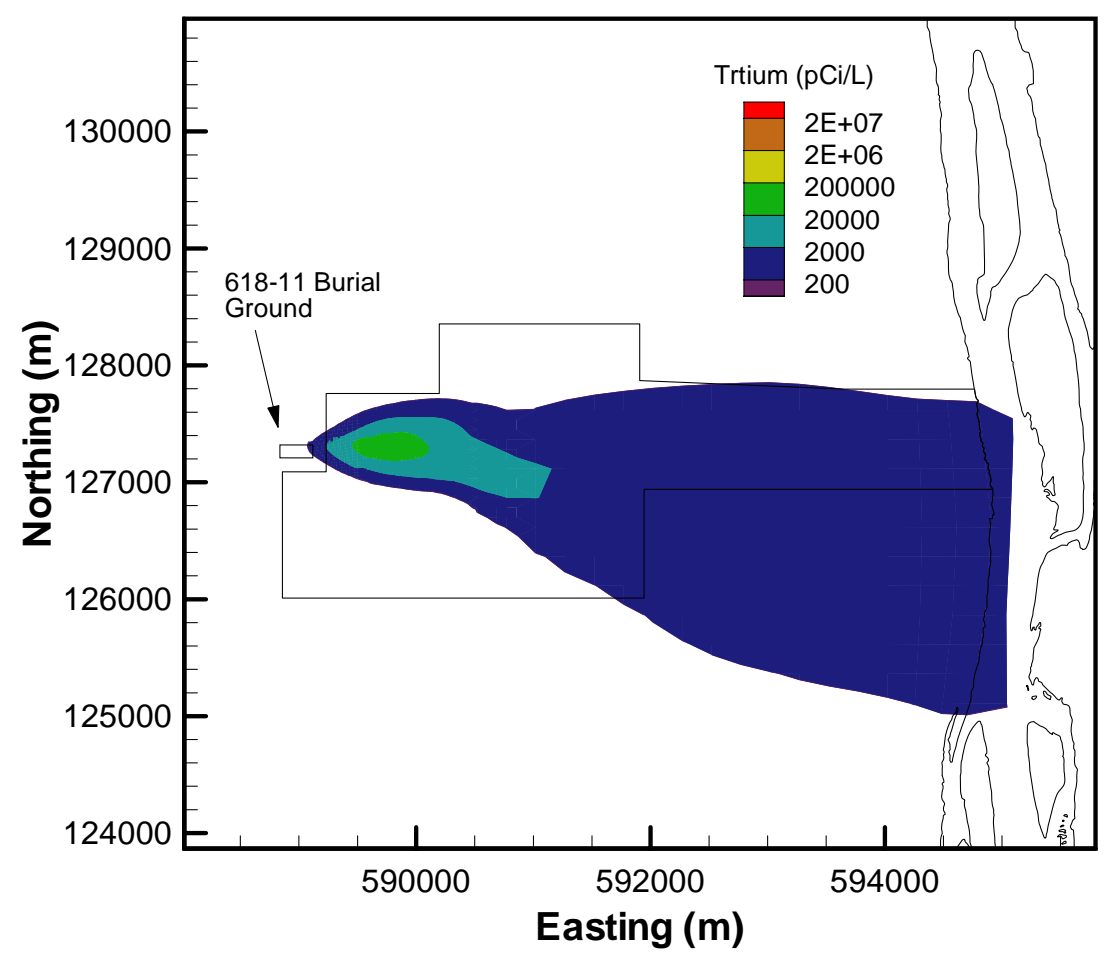

Figure 4.19. Tritium Plume at the 618-11 Burial Ground in 2017

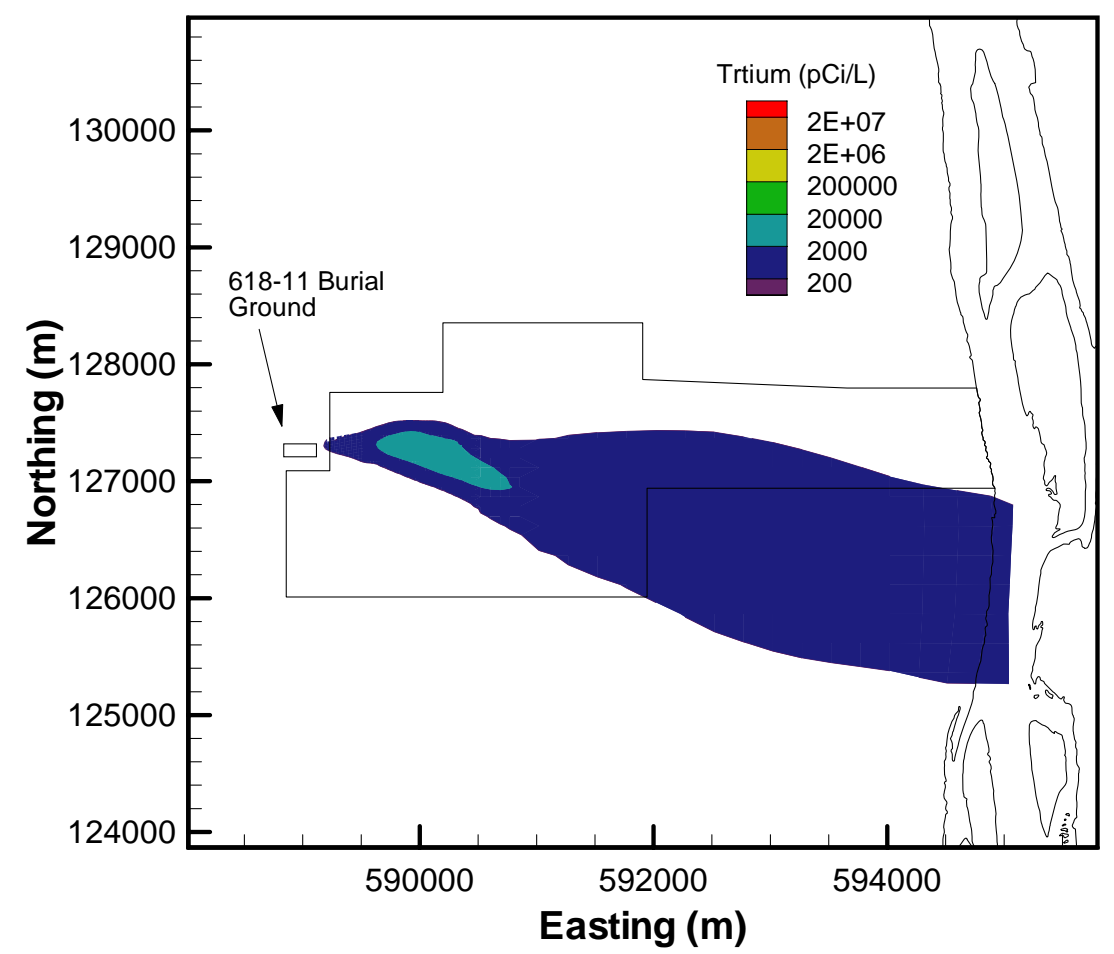

Figure 4.20. ' at the 618-11 Burial Ground in 2027, 3 Years after the Maximum Tritium Concentration Falls Below the Drinking Water Standard 


\subsection{Progress During Interim Remedial Action}

This section presents a discussion of the monitoring results that have become available during the approximately the past 12 years, i.e., since the remedial investigation and the original ROD for the 300-FF-5 Operable Unit (EPA 1996) and its subsequent update to include two additional outlying areas (EPA 2000). The discussion focuses on the ROD requirement to continue monitoring during a period of interim remedial action (i.e., prior to selecting a final remedy for groundwater) to verify that concentrations for contaminants of concern continue to decrease, as anticipated from the results of the remedial investigation (DOE 1995). A second focus for the discussion is the performance of the interim remedy in light of EPA guidance for including MNA as a remedy. ${ }^{1}$

Groundwater investigations associated with the 300-FF-5 Operable Unit are conducted under an operation and maintenance plan (DOE 2002b). This document was revised in response to Action Item 300-4 contained in the first 5-year review of the ROD (EPA 2001). The operable unit currently includes groundwater beneath the 300 Area, 618-11 burial ground, and 618-10 burial ground and adjacent former 316-4 cribs (see Figure 1.2). (Note: The 316-4 cribs and surrounding soil were removed in October 2004, with a second phase of soil excavation in December 2004. Backfilling of the excavation has not yet occurred, as of March 2005.)

\subsection{Operable Unit Monitoring and Characterization}

Groundwater monitoring for the 300 Area sub-region of the operable unit included semiannual sampling at monitoring wells during December and June. The intent was to characterize average seasonal conditions (December) and the period of high water-table conditions (June), which are caused by high discharge of the Columbia River during the spring (see Figures 2.1 through 2.6). Along the 300 Area shoreline, sampling and analysis at various sites and of various media are being conducted under several programs, with primarily annual sampling. Figure 1.2 provides an index map to shoreline monitoring locations:

- Under CERCLA, near-river wells, shoreline aquifer tubes, and riverbank springs are sampled to provide data on the distribution and concentration trends of contaminants in groundwater near the point-of-discharge to the river ecosystem.

- Under the Public Safety and Resource Protection Program, an integrated effort involving the Surface Environmental Surveillance Project and the Ecological Monitoring and Compliance Project is producing additional data on contaminants at the groundwater/river interface.

- Media sampled include riverbed pore water and sediment, river water, and various aquatic biota.

- To date, clams have been shown to be good indicators of locations where uranium-contaminated groundwater discharges to the riverbed (Patton et al. 2003).

\footnotetext{
${ }^{1}$ At the time the ROD (EPA 1996) was prepared, MNA had not yet been formally defined as a remedial action alternative by EPA. The guidance for doing so was published in 1999 .
} 
- Research on the interaction between groundwater and surface water at the 300 Area (and also the 100-N Area) was initiated by the Remediation Science and Closure Project (formerly known as Science and Technology) and continues during 2005.

- The 300 Area is included as part of the River Corridor Baseline Risk Assessment, which started in 2004.

For the outlying sub-regions (referred to as 300-FF-5 North in the scheduling database), monitoring the tritium plume that extends downgradient of the 618-11 burial ground continued with primarily quarterly sampling of wells used to define the plume (see Figure 1.2). Most sampling and analysis is conducted under CERCLA to meet the requirements for the 300-FF-5 Operable Unit, but some requirements are also associated with the 200-PO-1 Operable Unit that is defined by the site-wide plume that originated at the 200 East Area. An updated characterization of the 618-11 tritium plume is being prepared to support the (a) focused feasibility study for 300-FF-5 Operable Unit, and (b) the 5-year review of the ROD for the operable unit; both activities are in progress during 2005. At the 316-4 cribs/ 618-10 burial ground sub-region, the routine quarterly monitoring frequency was increased during late FY 2004 to better detect any potential changes associated with excavation of the 316-4 cribs. Remedial action associated with the cribs began near the end of FY 2004 with site preparation activities (actual excavation began in October 2004).

Near the 618-10 burial ground, an earlier soil-gas investigation around the southeastern perimeter fence was conducted in September 2002 as part of planning for two new monitoring wells, 699-S6-E4K and 699-S6-E4L (Williams et al. 2003). Slightly elevated helium-3/helium-4 ratios were observed at several soil-gas sample sites and were attributed to tritium in the site-wide plume, whose leading edge is in this area. Subsequent quarterly sampling of the two new wells during 2004 has not revealed any evidence for a groundwater plume whose origin is the burial ground. Water-quality conditions at those wells reflect the site-wide plume from 200-East Area sources, which contains co-contaminants nitrate, technetium-99, and iodine-129.

Numerous activities were initiated during FY 2004 to provide updated information on contamination in the subsurface at the 300 Area and outlying waste sites. These activities are part of extending the scope of the original remedial investigation (i.e., DOE 1995) to provide more information on what controls the persistence of the 300 Area uranium plume and how contaminant levels have changed during the years since the initial ROD (EPA 1996). Several projects were started to develop computer simulations for groundwater flow and contaminant transport in the 300 Area. An update to the original focused feasibility study for remedial action alternatives for groundwater was also started. Published reports containing the results of these investigations will become available during 2005.

\subsection{Interim Remedial Action Objectives}

The following is a status report on how well the interim remedial action objectives for COC or COPC are being met for the 300-FF-5 Operable Unit. COC for the 300 Area sub-region, as identified in the ROD (EPA 1996) are uranium, trichloroethene, and cis-1,2-dichloroethene. COPC for the 300-FF-5 North sub-regions, as identified in the explanation of significant differences (EPA 2000), are tritium at the 618-11 waste site, and uranium and tributyl phosphate at the 316-4/618-10 waste sites. Additional contamination indicator constituents are being tracked at each of these operable unit sub-regions. 


\subsubsection{Interim Action Requirements}

The ROD for the operable unit (EPA 1996) describes the selected interim action remedy as:

- "Continued monitoring of groundwater that is contaminated above health-based levels to ensure that concentrations continue to decrease.

- Institutional controls to ensure that groundwater use is restricted to prevent unacceptable exposures to groundwater contamination.”

The implementation of the interim remedy is described in the updated and expanded operations and maintenance plan (DOE 2002b) and a sampling and analysis plan (DOE 2002a). Key elements of the remedy include (a) continued monitoring of groundwater to verify previously modeled predictions of contaminant attenuation and (b) evaluating the need for active remedial measures. Continued monitoring involves sampling and analysis of groundwater collected from wells and shoreline sites, and also developing new information on (1) the re-supply of uranium to the 300 Area plume because of release from the vadose zone and/or aquifer solids, (2) the characteristics of discharge from the aquifer to the Columbia River, and (3) dispersal of contaminants in the river environment via biotic pathways.

As stated in the Executive Summary of the revised operations and maintenance plan (DOE 2002b), specific monitoring objectives include the following items:

- "Verify that natural attenuation reduces groundwater contamination concentrations to drinking water maximum contaminant levels over a reasonable time period.

- Confirm that contaminant concentrations in the river seeps do not exceed ambient water quality criteria or established remediation goals (drinking water standards).

- Validate contaminant fate and transport conceptual models.”

The CERCLA process includes a requirement to review the effectiveness of a ROD every 5 years, if contamination remains in the operable unit. The results of the first 5-year review for 300-FF-1 and 300-FF-5 Operable Units (EPA 2001) indicated that the remedial actions at 300 Area source waste sites were proceeding in an effective manner to protect human health and the environment. EPA's review re-affirmed that the cleanup goals and remedy selection for groundwater were still appropriate at the time the first 5-year review was released (April 2001). The next 5-year review will be conducted during 2005 and made available to the public by spring 2006.

The sections below provide a summary status for COC and COPC grouped by the three sub-regions within the 300-FF-5 Operable Unit. These sub-regions are the 300 Area, 618-11 burial ground, and 316-4 cribs/618-10 burial ground. The status is presented relative to the monitoring objectives outlined above.

\subsubsection{Interim Action: 300 Area}

The COC for the 300 Area, as identified in the ROD (EPA 1996), are uranium, trichloroethene, and cis-1,2-dichloroethene. 
- Objective: Verify that natural attenuation reduces groundwater contamination concentrations to drinking water maximum contaminant levels over a reasonable time period.

- Since 1998 , uranium concentrations at many monitoring wells have shown a declining trend, although not necessarily to the 30- $\mu \mathrm{g} / \mathrm{L}$ standard or lower (see trend charts in Appendix A). Investigation of changes in the areal extent, contaminated volume, and mass of dissolved uranium are consistent with the generally constant or declining concentration trends for recent years (see Chapter 2).

- New information from research on the mobility of uranium in 300 Area sediment indicates that (a) uranium sorption to vadose zone and aquifer solids is greater than previously known, and (b) that slow release of sorbed uranium to groundwater may continue for a long period of time (see Chapter 3). Whether the continued release of uranium from soil will result in long-term uranium concentrations remaining above the drinking water standard in the groundwater is not known.

- Dispersion of the uranium plume over time by discharge into the river, and removal of uraniumcontaminated groundwater via a water supply well, have not caused a significant change in the areal extent of the plume. This reinforces the suggestion that there is a continued release of uranium into the plume by some process(es) that are not yet clearly defined.

- Maximum concentrations of trichloroethene are generally below the drinking water standard (5 $\mu \mathrm{g} / \mathrm{L})$ at 300 Area wells and have been at most wells during the past 12 years. Concentrations are also generally below the standard at newly installed aquifer tube sites along the shoreline. There are two exceptions for the most recent sampling events:

- Values above the standard were observed at well 399-1-7, located between the 316-5 process trenches and the Columbia River, during $2004(5.4 \mu \mathrm{g} / \mathrm{L})$ and $2003(7.2 \mu \mathrm{g} / \mathrm{L})$.

- $\quad$ A value of $6.8 \mu \mathrm{g} / \mathrm{L}$ was observed during 2004 at aquifer tube site AT-3-3, which is located along the downgradient groundwater flow path from the process trenches and well 399-1-7.

- The maximum concentrations of cis-1,2-dichloroethene are generally well below the drinking water standard $(70 \mu \mathrm{g} / \mathrm{L})$ or non-detected at all 300 Area wells and shoreline monitoring sites.

- The exception is the occurrence of cis-1,2-dichloroethene at one well (399-1-16B), which monitors the bottom of the unconfined aquifer between the 316-5 process trenches, the presumed source, and the Columbia River.

- Where cis-1,2-dichloroethene is detected at several other deep wells near the 316-5 process trenches, and at the aquifer tube site nearest well 399-1-16B (tube site AT-3-3), concentrations are very low, and typically less than $3 \mu \mathrm{g} / \mathrm{L}$.

- $\quad$ The concentration at well 399-1-16B has remained essentially constant since 1992, and in the range 120 to $190 \mu \mathrm{g} / \mathrm{L}$. Groundwater flow at the bottom of the unconfined aquifer may be much slower than at the top, which would contribute to the persistence of the cis1,2-dichloroethene contamination at this location.

- Objective: Confirm that contaminant concentrations in the river seeps do not exceed ambient water quality criteria or established remediation goals (drinking water standards).

- Uranium concentrations are above the drinking water standard at several riverbank spring locations. During 2003, the maximum observed value was $160 \mu \mathrm{g} / \mathrm{L}$ (Poston et al. 2004). The gross alpha associated with this uranium also exceeds the 15-pCi/L drinking water standard. Work is in progress during 2005 to characterize temporal trends at 300 Area spring sites. 
- The maximum concentration for trichloroethene in 2003 was $2.2 \mu \mathrm{g} / \mathrm{L}$, which is below the standard.

- Cis-1,2-dichloroethene was not detected in riverbank springs during 2003.

- Objective: Validate contaminant fate and transport conceptual models.

- The conceptual model for uranium that was developed during the remedial investigation in the early 1990s supported the belief that removal of contaminated soils at liquid waste disposal sites (e.g., 316-5 process trenches) would stop the re-supply of uranium to groundwater at a rate that would cause drinking water standards to be exceeded. Computer simulation of future plume behavior, which was conducted as part of the remedial investigation, predicted that uranium concentrations would decrease to below the drinking water standard in 3 to 10 years from 1993 (DOE 1995). This rate of decrease has not occurred. The assumption of no re-supply from potential sources, such as contaminated facilities, the vadose zone beneath liquid waste disposal sites, and/or some other features that caused uranium to be sequestered but still mobile under certain conditions, appears to have been unsupported. Thus, additional investigations and updated computer simulations have been undertaken to better understand the current and future behavior of the uranium plume.

\subsubsection{Interim Action: 618-11 Burial Ground}

The COPC for the 618-11 sub-region, as identified in the explanation of significant difference to the ROD (EPA 2000), is tritium. Other Hanford Site contamination indicators are detected, but are believed to come from sources other than the 618-11 burial ground, and are typically at concentrations below drinking water standards. These constituents include gross alpha and beta, technetium-99, uranium, and nitrate.

- Objective: Verify that natural attenuation reduces groundwater contamination concentrations to drinking water maximum contaminant levels over a reasonable time period.

- Tritium concentrations near the 618-11 burial ground show a decreasing trend since the occurrence of peak values during 2000. Current levels ( 2 M pCi/L) still greatly exceed the drinking water standard of $20,000 \mathrm{pCi} / \mathrm{L}$. The decreasing concentrations observed close to the burial ground source cannot be attributed to radioactive decay alone (half-life is 12.3 years), thus indicating that transport processes are also involved. A likely scenario includes dispersal of a "pulse” release that was first identified in 1999 2000.

- Relatively constant or gradually increasing concentration trends are observed at wells along the downgradient flow path from the burial ground, also indicating a relatively slow downgradient migration of the tritium plume. Radioactive decay and dispersion are the primary natural causes for reducing tritium concentrations.

- Assuming no further input to the tritium plume, initial computer simulation results suggest that tritium concentrations within the plume will decrease to the drinking water standard within tens of years. Simulation runs are being made for a variety of scenarios, which will provide additional information regarding the accuracy and uncertainty associated with predictions.

- Radioactive decay causes an actual reduction in the amount of tritium in the plume, although the potential for re-supply by future releases from the burial ground is largely unknown. 
- Objective: Confirm that contaminant concentrations in the river seeps do not exceed ambient water quality criteria or established remediation goals (drinking water standards).

- There is no evidence to suggest that the tritium plume associated with the burial ground has or will reach the Columbia River. Initial modeling results support the conclusion that it is unlikely that the plume will reach the river at concentrations exceeding the drinking water standard.

- Objective: Validate contaminant fate and transport conceptual models.

- The conceptual model for the tritium plume, which was developed during the initial investigation of unexpectedly high concentrations in 1999 (Dresel et al. 2000), did not include a detailed analysis of how the plume was likely to evolve. Some computer simulation work was subsequently conducted to provide information on whether the plume might reach the river at levels of concern, ${ }^{2}$ but results were inconclusive. Current simulation results, and trends revealed by five additional years of monitoring results, provide evidence that the plume will not reach the river at levels of concern. Continued monitoring will be used to validate the new fate and transport modeling being conducted during 2005.

\subsubsection{Interim Action: 316-4 Cribs/618-10 Burial Ground}

The following is a brief status of how well the monitoring objectives for COC are being met at the 316-4/618-10 waste sites sub-region. COPC, as identified in the explanation of significant difference to the ROD (EPA 2000) are uranium and tributyl phosphate, which are associated with the 316-4 cribs. No COPC have yet been associated with the 618-10 burial ground. Other Hanford Site contamination indicators are detected, but are associated with the site-wide plume whose origin is the 200 East Area, and at concentrations below drinking water standards. These constituents include tritium, technetium-99, and nitrate.

- Objective: Verify that natural attenuation reduces groundwater contamination concentrations to drinking water maximum contaminant levels over a reasonable time period.

- Most monitoring results for uranium at wells near the 316-4 cribs are below the $30-\mu \mathrm{g} / \mathrm{L}$ drinking water standard:

- $\quad$ The exceptions are recent results at wells 699-S6-E4A and 699-S6-E4L (Figure 2.10), which show a recent rise in concentrations.

- A cause for these increases is not clear; the increases appear to have started prior to initiation of excavation activities at the 316-4 cribs site, so remobilization of contaminants because of that activity seems unlikely.

- $\quad$ More frequent monitoring at 699-S6-E4L, and also at 699-S6-E4A when logistically possible, is being conducted as remedial action at the 316-4 cribs waste site proceeds during 2005.

\footnotetext{
${ }^{2}$ Letter report from PE Dresel and MP Bergeron (Pacific Northwest National Laboratory) to KM Thompson and MJ Furman (U.S. Department of Energy, Richland, Washington), Evaluation of the Impact of Tritium Contamination in Groundwater from the 618-11 Burial Ground at the Hanford Site, dated December 20, 2001.
} 
- Tributyl phosphate was not detected in groundwater in this sub-region during FY 2004, although it had been detected in 1995 and 1996 at 699-S6-E4A during well refurbishment activities. However, elevated concentrations of this relatively immobile organic compound were encountered (along with uranium) in the vadose zone during excavation of the 316-4 cribs in October and December 2004.

- Objective: Confirm that contaminant concentrations in the river seeps do not exceed ambient water quality criteria or established remediation goals (drinking water standards).

- There is no conclusive evidence to indicate that potentially contaminated groundwater caused by releases from the 316-4/618-10 waste sites has reached the Columbia River.

- Objective: Validate contaminant fate and transport conceptual models.

- No groundwater plumes are currently documented or mapped as having been created by releases associated with the 316-4 cribs and/or the 618-10 burial ground, although plumes may have been generated in the past (i.e., during the 1950s and 1960s) as a consequence of active disposal to the cribs. A possibility exists that releases impacting groundwater during the operations period did occur, but these potential impacts have not been detected by subsequent monitoring activities. Therefore, no groundwater flow and/or contaminant transport modeling is currently underway for this sub-region.

\subsection{EPA Guidelines for Monitored Natural Attenuation as a Remedy}

Formal EPA guidance for including Monitored Natural Attenuation (MNA) as a remedial action alternative was not available at the time of the 300-FF-5 Operable Unit ROD (EPA 1996). However, the selected remedy described in that decision can be evaluated in the context of the formal guidance that was subsequently provided in the OSWER MNA Directive (EPA 1999). It may also be informative to consider whether continuation of an MNA remedy would be reasonable, based on the updated information concerning concentrations and trends for 300-FF-5 Operable Unit COC and COPC presented in earlier Chapters (i.e., Chapters 2, 3, and 4). The following sections discuss what has evolved during the period of interim action, and how COC and COPC might be viewed in the context of current MNA guidance. Statements made should not be viewed as recommendations for remedial action alternatives; that will be developed as described in the work plan for the Phase III Feasibility Study (DOE 2005). In the following discussion, direct quotes from the OSWER MNA Directive are presented in italics.

The natural attenuation processes recognized in the OSWER MNA Directive are described in the following quotation:

The "natural attenuation processes" that are at work in such a remediation approach [MNA] include a variety of physical, chemical, or biological processes that, under favorable conditions, act without human intervention to reduce the mass, toxicity, mobility, volume, or concentration of contaminants in soil or groundwater. These in-situ processes include biodegradation; dispersion; dilution; sorption; volatilization; radioactive decay; and chemical or biological stabilization, transformation, or destruction of contaminants. 


\subsubsection{Key Principals for Selection of Remedial Measures}

The OSWER MNA Directive lists four key principles that should be considered in selection of remedial measures, including MNA. These principles and a discussion of how the 300-FF-5 remedy addresses each are provided in the following paragraphs:

- Source control measures should use treatment to address "principal threat" wastes wherever practicable, and engineering controls such as containment for waste that poses a relatively low longterm threat or where treatment is impracticable.

- The principal liquid and solid waste disposal sites that were the sources for 300 Area COC have been, or are soon to be, remediated (i.e., removal of facilities and the adjacent soils) as part of remedial action in the 300-FF-1 Operable Unit. The 618-10 and 618-11 burial grounds, which are contained in the 300-FF-2 Operable Unit, will be remediated by removal actions that are currently in the planning stage. These remedial actions will reduce or eliminate primary sites of contamination that posed a potential threat of groundwater impacts.

- Contaminated groundwaters should be returned to "their beneficial uses wherever practicable, within a timeframe that is reasonable given the particular circumstances of the site." When restoration of groundwater is not practicable, EPA "expects to prevent further migration of the plume, prevent exposure to the contaminated groundwater, and evaluate further risk reduction."

- In the 300-FF-5 ROD (EPA 1996) remedy selection, natural attenuation processes were expected to continue to cause decreasing COC concentrations, such that concentrations below the drinking water standard could be reached within a reasonable time. Meeting the drinking water standard would return the aquifer to beneficial uses.

- At the time of the ROD, it was predicted that the drinking water standards would be reached in 3 to 10 years. While this rate of concentration decrease has not occurred for uranium and cis-1,2-dichloroethene, recent efforts have re-examined the rate of decrease. These new results, along with additional characterization data and an updated fate and transport model, can be used to assess whether the COC will decrease to target levels in a "reasonable time." In the OSWER MNA Directive, EPA states that it expects MNA to achieve remediation objectives within a time frame that is reasonable compared to other alternatives. This type of comparison can be conducted in the framework of the planned feasibility study (DOE 2005). Exposure to groundwater while concentrations of COC are above standards is readily addressed through institutional controls because the operable unit is under administrative control of the government.

- For the 300-FF-5 ROD (EPA 1996) remedy selection, it was determined that the COC plumes were not presenting a risk to receptors in the Columbia River.

- A specific remedial action objective from the 1996 ROD is to "Protect the Columbia River such that contaminants in the groundwater or remaining in the soil after remediation do not result in an impact to the Columbia River that could exceed the Washington State Surface Water Quality Standards.” The 1996 ROD states that this objective will be achieved by “...preventing further degradation of the groundwater quality in the uranium plume such that receptors that may be affected at the groundwater discharge point to the Columbia River are not subject to any additional incremental adverse risks.” Thus, the contaminant concentrations in the groundwater in 1996 were deemed acceptable, albeit with the expectation that these concentrations would decrease over time. 
- Characterization and analysis of the tritium plume at the 618-11 burial ground has shown that this plume is a good candidate for considering MNA as a remedial action alternative. This plume was not part of the 1996 ROD. However, recent data collection and analysis have shown that natural attenuation by radioactive decay and dilution/dispersion processes is expected to occur. Preliminary numerical modeling results for the plume under these attenuation conditions suggest that the current tritium concentrations in the 618-11 tritium plume will decrease to below the drinking water standard within several tens of years.

- Contaminated soil should be remediated to achieve an acceptable level of risk to human and environmental receptors, and to prevent any transfer of contaminants to other media (e.g., surface or groundwater, air, sediments) that would result in an unacceptable risk or exceed required cleanup levels.

- Liquid and solid waste discharge sites that were the source for 300 Area COC are being remediated as part of remedial action in the 300-FF-1 Operable Unit. The 618-10 and 618-11 burial grounds will be addressed as part of remedial actions in the 300-FF-2 Operable Unit. This remediation will likely remove or somehow isolate the primary areas of soil contamination associated with waste disposal sites. These actions will prevent impacts on groundwater quality and will meet 300-FF-1 and 300-FF-2 goals regarding other receptors.

- For the 300 Area remedy selection in the 1996 ROD, it was determined that the COC groundwater plumes were not presenting a risk to receptors in the Columbia River and that reduction in the contaminated soil as a part of surface site remedial action would further reduce contaminant levels in groundwater and surface water. Additional study of the uranium contamination in the vadose zone and aquifer sediment indicated that uranium adsorbs to these solids to a greater extent than previously expected. The rates of adsorption and related desorption processes will impact the time over which groundwater remains contaminated. Geochemical processes are therefore a key controlling element in assessing the timeframe for natural attenuation as well as other remedial action alternatives.

- Remedial actions in general should include opportunity(ies) for public involvement that serve to both educate interested parties and to solicit feedback concerning the decision making process.

- All 300-FF-5 Operable Unit actions have been, and will continue to be, conducted in accordance with CERCLA guidance that includes public participation in the decision-making process.

\subsubsection{Approach for Evaluating Monitored Natural Attenuation as a Remedy}

The OSWER MNA Directive outlines a three-tiered approach for evaluating the suitability of MNA as a remedy. The three tiers of information, or "lines of evidence," included in this approach are presented below, along with a discussion of the site-specific information for the 300-FF-5 Operable Unit.

1. Historical groundwater and/or soil chemistry data that demonstrate a clear and meaningful trend of decreasing contaminant mass and/or concentration over time at appropriate monitoring 
or sampling points. (In the case of a groundwater plume, decreasing concentrations should not be solely the result of plume migration. In the case of inorganic contaminants, the primary attenuating mechanism should also be understood.)

- Data in this report (Chapter 2) frequently reveal essentially constant or decreasing trends during recent years for the uranium and tritium plumes that are still present above action levels, i.e., currently the drinking water standard. A localized area involving a known source site, single monitoring well, and possibly an aquifer tube site at the shoreline within the 300 Area has exhibited stable concentrations of cis-1,2-dichloroethene that exceed standards over the duration of the remedial investigation in the operable unit. Attenuation mechanisms for the inorganic contaminants are as follows:

- (1) For 300 Area uranium contamination, dilution, dispersion, and the slow desorption rate of uranium from vadose zone and aquifer solids, are mechanisms working to reduce concentrations in the aquifer and at locations of discharge to the river ecosystem. These mechanisms were all recognized as contributors during the remedy selection in the 1996 ROD.

- (2) For 618-11 tritium contamination, radioactive decay and dilution/dispersion mechanisms are reducing contaminant concentrations, as revealed by recent monitoring results and data analysis.

2. Hydrogeologic and geochemical data that can be used to demonstrate indirectly the type(s) of natural attenuation processes active at the site, and the rate at which such processes will reduce contaminant concentrations to required levels. For example, characterization data may be used to quantify the rates of contaminant sorption, dilution, or volatilization, or to demonstrate and quantify the rates of biological degradation processes occurring at the site.

- Selection of the remedy in the ROD (EPA 1996) was based on use of conceptual site model and computer simulations that were done as part of the remedial investigation (DOE 1995). Recently, site-specific geochemical data have been collected to better quantify the geochemical processes occurring at the site. New information on uranium complexation and the related controls on mobility has evolved. This geochemical process information and recent site hydrogeologic data are being incorporated in new computer simulations for the 300 Area uranium plume, which will be used to provide predictions of contaminant fate and transport. These models and the underlying data are available to support evaluation of MNA as a remedy. Additional characterization data to verify the updated conceptual site model for the uranium plume (Chapter 3), in particular with respect to the distribution of uranium in soils that can contact the groundwater, may also be needed as part of an MNA evaluation.

3. Data from field or microcosm studies (conducted in or with actual contaminated site media) which directly demonstrate the occurrence of a particular natural attenuation process at the site and its ability to degrade the contaminants of concern (typically used to demonstrate biological degradation processes only).

- Site-specific laboratory tests have been completed that quantify uranium sorption/ desorption processes that impart significant influence on the concentration of uranium in groundwater. Numerical modeling can be used to demonstrate the impact of this sorption behavior on uranium concentration over time within the plume. 


\subsubsection{Key Considerations as to Appropriateness of MNA at a Particular Site}

The OSWER MNA Directive provides a list of key considerations for determining whether MNA is appropriate to evaluate as a remedial action alternative for a site. These considerations are discussed below in the context of re-evaluating the current interim remedial action remedy contained in the 1996 ROD, given the subsequent MNA guidance. The considerations are also relevant for discussing the potential for MNA as a continuing remedy for the 300-FF-5 COC and COPC.

1. Whether the contaminants present in soil or groundwater can be effectively remediated by natural attenuation processes.

- At the time of the 1996 ROD, natural attenuation processes were deemed sufficient to result in acceptable concentrations of COC discharging into the Columbia River. Trend data reported herein show a generally decreasing trend for all COC except for a localized area of cis-1,2-dichloroethene that has a stable concentration.

2. Whether or not the contaminant plume is stable and the potential for the environmental conditions that influence plume stability to change over time.

- $\quad$ Trend data reported herein show a decreasing trend for all COC except for a localized area of cis-1,2-dichloroethene that has a stable concentration. There is no expected change in environmental conditions over time that would impact this trend other than short duration events associated with soil disturbance during source area excavation and periods of time when the groundwater table is unusually high because of high river stage conditions.

3. Whether human health, drinking water supplies, other groundwaters, surface waters, ecosystems, sediments, air, or other environmental resources could be adversely impacted as a consequence of selecting MNA as the remediation options.

- $\quad$ No resources have been shown to be adversely impacted (other than the aquifer) during the timeframe over which natural attenuation processes are reducing the COC concentrations to drinking water standards. Institutional controls are limiting groundwater use during this time and there is currently no significant demand for using the aquifer as a drinking water supply.

4. Current and projected demand for the affected resource over the time period that the remedy will remain in effect.

- There is no projected demand for the groundwater over the expected time period of the remedy, other than continued use of groundwater for laboratory aquarium purposes, which is anticipated to continue through 2009. 
5. Whether the contamination, either by itself or as an accumulation with other nearby sources (onsite or offsite), will exert a long-term detrimental impact on available water supplies or other environmental resources.

- $\quad$ There is no expected long-term detrimental impact by the COC on any resources.

6. Whether the estimated timeframe of remediation is reasonable compared to timeframes required for other more active methods (including anticipated effectiveness of various remedial approaches on different portions of the contaminated soil and/or groundwater).

- $\quad$ At the time of the 1996 ROD, the time frame for natural attenuation processes was deemed reasonable. The reasonableness of the timeframe associated with continuing MNA as a remedy will be assessed as part of re-evaluating the interim remedial action remedy (DOE 2005).

7. The nature and distribution of sources of contamination and whether these sources have been, or can be, adequately controlled.

- The primary sources of contamination at the previous waste disposal areas have been, or are soon to be, addressed by removal of facilities and excavation of adjacent soils.

8. Whether the resulting transformation products present a greater risk, due to increased toxicity and/or mobility, than do the parent contaminants.

- There are no transformation products that are producing a greater risk than the parent compounds. It is not known whether cis-1,2-dichloroethene is a daughter product or a directly disposed contaminant, but the localized plume is not currently, and is not expected to be, a risk.

9. The impact of existing and proposed active remediation measures upon the MNA component of the remedy, or the impact of remediation measures or other operations/activities (e.g., pumping wells) in close proximity to the site.

- $\quad$ There may be short-term variations in groundwater concentrations during source excavation actions, but these do not impact the overall performance of the current remedy and would not be expected to impact a continuing MNA remedy.

10. Whether reliable site-specific mechanisms for implementing institutional controls (e.g., zoning ordinances) are available, and if an institution responsible for their monitoring and enforcement can be identified.

- Exposure to groundwater while concentrations of COC are above standards is readily addressed through institutional controls because the operable unit is under administrative control of the government, and likely to remain so for tens of years at a minimum (DOE 1999).

Based on the above information, the interim remedial action described in the 1996 300-FF-5 ROD, i.e., monitoring with institutional controls, appears to be consistent with implementing MNA as a remedy. It is also evident that MNA should be included as part of re-evaluating the remedy for the $300-\mathrm{FF}-5$ Operable Unit. 


\subsection{References}

Campbell MD. 1994. Monitoring Groundwater and River Interaction Along the Hanford Reach of the Columbia River. PNL-9437, Pacific Northwest Laboratory, Richland, Washington.

Campbell MD and DR Newcomer. 1992. Automatic Measurement of Water Levels Within the 300-FF-5 Boundary. PNL-7874, Pacific Northwest Laboratory, Richland, Washington.

Cole CR, MP Bergeron, CJ Murray, PD Thorne, SK Wurstner, and P Rogers. 2001. Uncertainty Analysis Framework - Hanford Site-Wide Groundwater Flow and Transport Model. PNNL-13641, Pacific Northwest National Laboratory, Richland, Washington.

Comprehensive Environmental Response, Compensation, and Liability Act. 1980. Public Law 96-150, as amended, 94 Stat. 2767, 42 USC 9601 et seq.

Davis JA, DL Bond, and P Fox. 2004. "Labile U(VI) and Progress Toward a Generalized Surface Complexation Model," presented at a workshop on Conceptual Model Development and Reactive Transport Modeling for the 300 Area Uranium Plume in 300-FF-5, held May 10-11, 2004, Richland, Washington. Available at http://www.hanford.gov/cp/gpp/public/workshops.cfm.

DeFord DH, RW Carpenter, and MW Einan. 1994. 300-FF-2 Operable Unit Technical Baseline Report. BHI-00012, Rev. 00, Bechtel Hanford, Inc., Richland, Washington.

Demiter JA and WO Greenhalgh. 1997. Characterization of the 618-11 Solid Waste Burial Ground, Disposed Waste, and Description of the Waste-Generating Facilities. HNF-EP-0649, prepared for Waste Management Federal Services, Inc., Richland, Washington.

Deutsch CV and AG Journel. 1998. GSLIB: Geostatistical Software Library and User's Guide. Oxford University Press, New York.

DOE. 1988. "Hydrology” Volume II, Chapter 3 in Site Characterization Plan. DOE/RW-0164, U.S. Department of Energy, Washington, D.C.

DOE. 1994. Phase 1 Remedial Investigation Report for the 300-FF-5 Operable Unit. DOE/RL-93-21, Rev. 0, Volumes 1 and 2, U.S. Department of Energy, Richland, Washington.

DOE. 1995. Remedial Investigation/Feasibility Study Report for the 300-FF-5 Operable Unit.

DOE/RL-94-85, U.S. Department of Energy, Richland, Washington.

DOE. 1999. Hanford Comprehensive Land-Use Plan Environmental Impact Statements.

DOE/EIS-0222-F, U.S. Department of Energy, Richland, Washington.

DOE. 2002a. 300-FF-5 Operable Unit Sampling and Analysis Plan. DOE/RL-2002-11, Rev. 0. Prepared by CH2M HILL Hanford, Inc. for the U.S. Department of Energy, Richland, Washington. 
DOE. 2002b. Operation and Maintenance Plan for the 300-FF-5 Operable Unit. DOE/RL-95-73, Rev. 1. Prepared by CH2M HILL Hanford, Inc. for the U.S. Department of Energy, Richland, Washington.

DOE. 2002c. Standardized Stratigraphic Nomenclature for Post-Ringold-Formation Sediments Within the Central Pasco Basin. DOE/RL-2002-39, U.S. Department of Energy, Richland, Washington.

Dresel PE, BA Williams, JC Evans, RM Smith, CJ Thompson, and LC Hulstrom. 2000. Evaluation of Elevated Tritium Levels in Groundwater Downgradient from the 618-11 Burial Grounds Phase I Investigations. PNNL-13228, Pacific Northwest National Laboratory, Richland, Washington.

EPA. 1996. Record of Decision for USDOE Hanford 300-FF-1 and 300-FF-5 Operable Units Remedial Actions. Agreement Between U.S. Department of Energy and U.S. Environmental Protection Agency, with Concurrence by the Washington State Department of Ecology, July 17, 1996.

EPA. 1999. Use of Monitored Natural Attenuation at Superfund, RCRA Corrective Action, and Underground Storage Tank Sites. OSWER Directive 9200.4-17, U.S. Environmental Protection Agency, Washington, D.C.

EPA. 2000. Explanation of Significant Difference for the 300-FF-5 Record of Decision (see EPA 1996 for original ROD). Agreement between U.S. Department of Energy and U.S. Environmental Protection Agency, with concurrence by the Washington State Department of Ecology, June 2000. (adds groundwater beneath the 618-10 and 618-11 Burial Grounds to the 300-FF-5 Operable Unit)

EPA. 2001. USDOE Hanford Site: First Five Year Review Report. April 2001. Prepared by U.S. Environmental Protection Agency, Region 10, Hanford Project Office, Richland, Washington.

Fayer MJ and TB Walters. 1995. Estimated Recharge Rates at the Hanford Site. PNL-10285, Pacific Northwest Laboratory, Richland, Washington.

Gaylord DR and EP Poeter. 1991. Geology and Hydrology of the 300 Area and Vicinity, Hanford Site, South Central Washington. WHC-EP-0500, Westinghouse Hanford Company, Richland, Washington.

Gerber MS. 1992. Past Practices Technical Characterization Study - 300 Area -Hanford Site. WHC-MR-0388, Westinghouse Hanford Company, Richland, Washington.

Hartman MJ (ed.). 2000. Hanford Site Groundwater Monitoring: Setting, Sources, and Methods. PNNL-13080, Pacific Northwest National Laboratory, Richland, Washington.

Hartman MJ, LF Morasch, and WD Webber (eds.). 2005. Hanford Site Groundwater Monitoring for Fiscal Year 2004. PNNL-15070, Pacific Northwest National Laboratory, Richland, Washington.

Isaaks EH and RM Srivastava. 1989. An Introduction to Applied Geostatistics. Oxford University Press, New York.

Lindberg JW and FW Bond. 1979. Geohydrology and Ground-Water Quality Beneath the 300 Area, Hanford Site, Washington. PNL-2949, Pacific Northwest Laboratory, Richland, Washington. 
Lindberg JW and CJ Chou. 2001. 300 Area Process Trenches Groundwater Monitoring Plan. PNNL-13645, Pacific Northwest National Laboratory, Richland, Washington.

Lindberg JW and RE Peterson. 2004. “300-FF-5 Operable Unit,” Chapter 2.12 in Hanford Site Groundwater Monitoring for Fiscal Year 2003, MJ Hartman, LF Morasch, and WD Webber (eds.), PNNL-14548, Pacific Northwest National Laboratory, Richland, Washington.

Lindberg JW and RE Peterson. 2005. “300-FF-5 Operable Unit,” Chapter 2.12 in Hanford Site Groundwater Monitoring for Fiscal Year 2004, MJ Hartman, LF Morasch, and WD Webber (eds.), PNNL-15070, Pacific Northwest National Laboratory, Richland, Washington.

Lindsey KA. 1995. Miocene- to Pliocene-Aged Suprabasalt Sediments of the Hanford Site, SouthCentral Washington. BHI-00184, Rev. 0, Bechtel Hanford, Inc., Richland, Washington.

Liu C, J Zachara, and N Qafoku. 2004. “Modeling of Uranium Sorption/Desorption with Advection,” presented at a workshop on Conceptual Model Development and Reactive Transport Modeling for the 300 Area Uranium Plume in 300-FF-5, held May 10-11, 2004, Richland, Washington. Available at http://www.hanford.gov/cp/gpp/public/workshops.cfm.

Meyer PD, M Ye, SP Neuman, and KJ Cantrell. 2004. Combined Estimation of Hydrogeologic Conceptual Model and Parameter Uncertainty. NUREG/CR-6843, U.S. Nuclear Regulatory Commission, Washington, D.C.

Moench AF. 1994. "Specific Yield as Determined by Type-Curve Analysis of Aquifer Test Data." Groundwater 32(6):949-957.

Murray CJ, Y Chien, and PD Thorne. 2004. A Geostatistical Analysis of Historical Field Data on Tritium, Technetium-99, Iodine-129, and Uranium. PNNL-14618, Rev. 0, Pacific Northwest National Laboratory, Richland, Washington.

Neuman SP and PJ Wierenga. 2003. A Comprehensive Strategy of Hydrogeologic Modeling and Uncertainty Analysis for Nuclear Facilities and Sites. NUREG/CR-6805, U.S. Nuclear Regulatory Commission, Washington, D.C.

Oostrom M, DH Meck, and MD White. 2003. STOMP Subsurface Transport Over Multiple Phases, Version 3.0, An Introductory Short Course. PNNL-14440, Pacific Northwest National Laboratory, Richland, Washington.

Patton GW, SP Van Verst, BL Tiller, EJ Antonio, and TM Poston. 2003. Survey of Radiological and Chemical Contaminants in the Near-Shore Environment at the Hanford Site 300 Area. PNNL-13692, Rev. 1, Pacific Northwest National Laboratory, Richland, Washington.

Poston TM, RW Hanf, RL Dirkes, LF Morasch (eds.). 2004. Hanford Site Environmental Report for Calendar Year 2003. PNNL-14687, Pacific Northwest National Laboratory, Richland, Washington.

Reidel SP, KA Lindsey, and KR Fecht. 1992. Field Trip Guide to the Hanford Site. WHC-MR-0391, Westinghouse Hanford Company, Richland, Washington. 
Resource and Conservation Recovery Act. 1976. Public Law 94-580, as amended, 90 Stat. 2795, 42 USC 6901 et seq.

Richmond MC, WA Perkins, and Y Chien. 2000. Numerical Model Analysis of System-Wide Dissolved Abatement Alternatives. Final report prepared for the U.S. Army Corps of Engineers, Walla Walla District, by Battelle Pacific Northwest Division, Richland, Washington.

Rockhold ML, MJ Fayer, CT Kincaid, and GW Gee. 1995. Estimation of Natural Groundwater Recharge for the Performance Assessment of a Low-Level Waste Disposal Facility at the Hanford Site. PNL-10508, Pacific Northwest National Laboratory, Richland, Washington.

Schalla R, RW Wallace, RL Aaberg, SP Airhart, DJ Bates, JVM Carlile, CS Cline, DI Dennison, MD Freshley, PR Heller, EJ Jensen, KB Olsen, RG Parkhurst, JT Rieger, and EJ Westergard. 1988. Interim Characterization Report for the 300 Area Process Trenches. PNL-6716, Pacific Northwest Laboratory, Richland, Washington.

Serne RJ, CF Brown, HT Schaef, EM Pierce, MJ Lindberg, Z Wang, P Gassman, and J Catalano. 2002. 300 Area Uranium Leach and Adsorption Project. PNNL-14022, Pacific Northwest National Laboratory, Richland, Washington.

Swanson LC, GG Kelty, KA Lindsey, KR Simpson, RK Price, and SD Consort. 1992. Phase 1 Hydrogeologic Summary of the 300-FF-5 Operable Unit, 300 Area. WHC-SD-EN-TI-052, Rev. 0, Westinghouse Hanford Company, Richland, Washington.

Thorne P D and DR Newcomer. 1992. Summary and Evaluation of Available Hydraulic Property Data for the Hanford Site Unconfined Aquifer System. PNL-8337, Pacific Northwest Laboratory, Richland, Washington.

Vermeul VR, MP Bergeron, CR Cole, CJ Murray, WE Nichols, TD Scheibe, PD Thorne, SR Waichler, and Y Xie. 2003. Transient Inverse Calibration of the Site-Wide Groundwater Flow Model (ACM-2): FY03 Progress Report. PNNL-14398, Pacific Northwest National Laboratory, Richland, Washington.

WAC 173-201A. "Surface Water Quality Standards.” Washington Administrative Code, Olympia, Washington.

Weiss SG. 2005. DQO Summary Report for the 100 Area and 300 Area Component of the RCBRA. BHI-01757, Rev. 0, Bechtel Hanford, Inc., Richland, Washington.

White MD and M Oostrom. 2000. STOMP, Subsurface Transport Over Multiple Phases. Version 2.0. Theory Guide. PNNL-12030, Pacific Northwest National Laboratory Report.

White MD and M Oostrom. 2004. STOMP Subsurface Transport Over Multiple Phases, Version 3.1, User's Guide. PNNL-14478, Pacific Northwest National Laboratory, Richland, Washington.

Williams BA, RE Peterson, and KB Olsen. 2003. Soil Gas Survey and Well Installation at the 618-10 Burial Ground, 300-FF-5 Operable Unit, Hanford Site, Washington. PNNL-14320, Pacific Northwest National Laboratory, Richland, Washington. 
Young JS and JS Fruchter. 1991. Addendum to Data Compilation Task Report for the Source Investigation of the 300-FF-1 Operable Unit Phase I Remedial Investigations. EMO-1026, prepared by Environmental Management Operations for the U.S. Department of Energy, Richland, Washington.

Zachara JM. 2004. "Batch Measurements of Desorption/Adsorption from Vadose Zone and Aquifer Sediments,” presented at a workshop on Conceptual Model Development and Reactive Transport Modeling for the 300 Area Uranium Plume in 300-FF-5, held May 10-11, 2004, Richland, Washington. Available at http://www.hanford.gov/cp/gpp/public/workshops.cfm.

Zachara, JM, JA Davis, C Liu, JP McKinley, N Qafoku, DM Wellman, and SB Yabusaki. 2005. Uranium Geochemistry in Vadose Zone and Aquifer Sediments from the 300 Area Uranium Plume. PNNL-15121, Pacific Northwest National Laboratory, Richland, Washington. 


\section{Appendix A}

\section{Trend Charts of Uranium Concentrations at 300 Area Wells}




\section{Appendix A}

\section{Trend Charts of Uranium Concentrations at 300 Area Wells}

This appendix contains trend charts showing uranium concentrations in wells at the 300 Area that monitor the uppermost part of the aquifer, i.e., the hydrologic unit that contains the uranium plume. The charts cover the period 1974 through 2004. The values plotted include measured uranium concentrations, results considered outliers (i.e., not representative of aquifer conditions), and representative values determined for two-year time windows. The latter values were used in the geostatistical analysis of uranium plume parameters.

Electronic copies of the 1992 water-table animation and uranium concentration trend charts shown in this appendix can be obtained by contacting R. E. Peterson at 509-373-9020, robert.peterson@pnl.gov, or by mail at: Pacific Northwest National Laboratory, P.O. Box 999 (MS K6-96), Richland, WA 99352. 

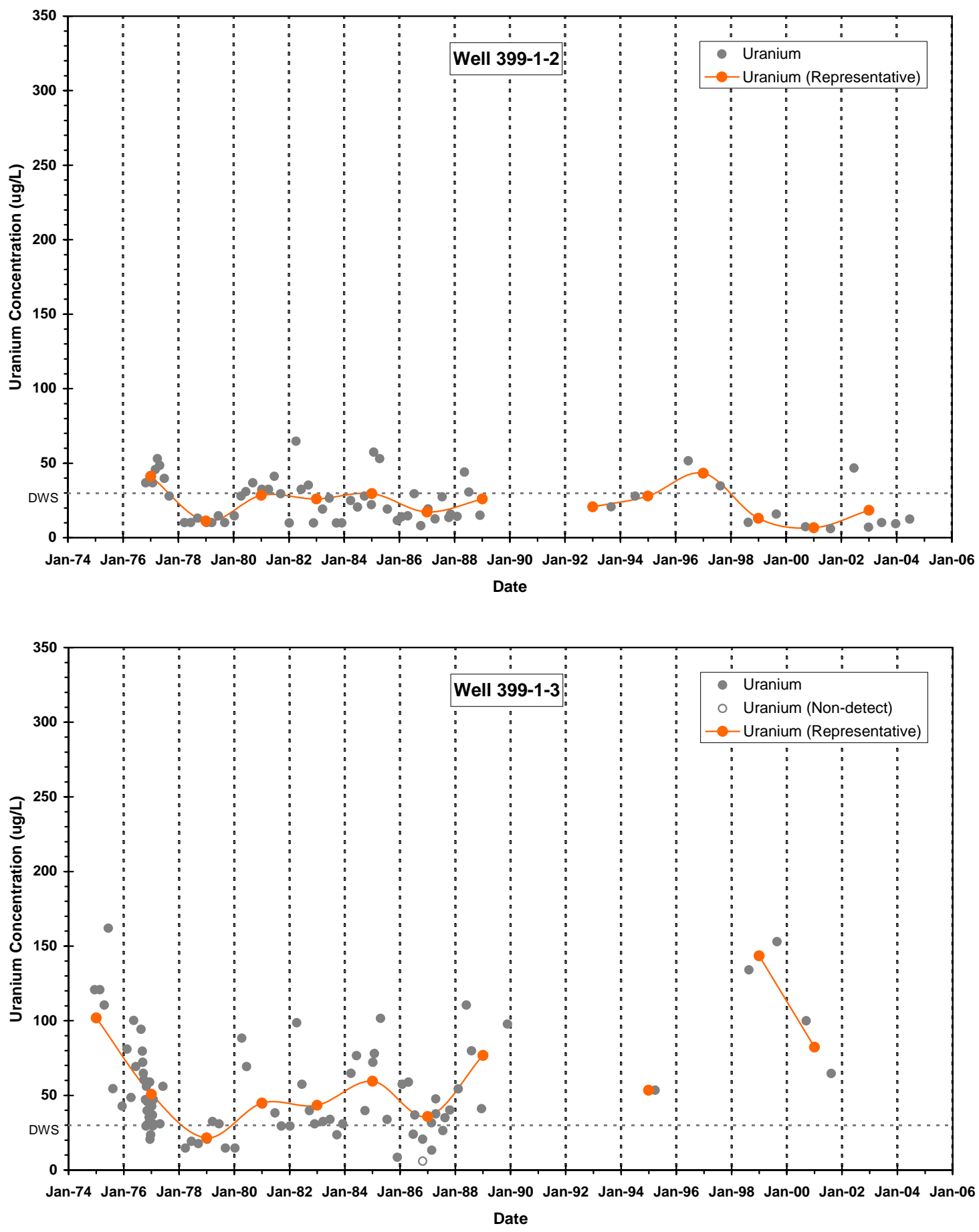

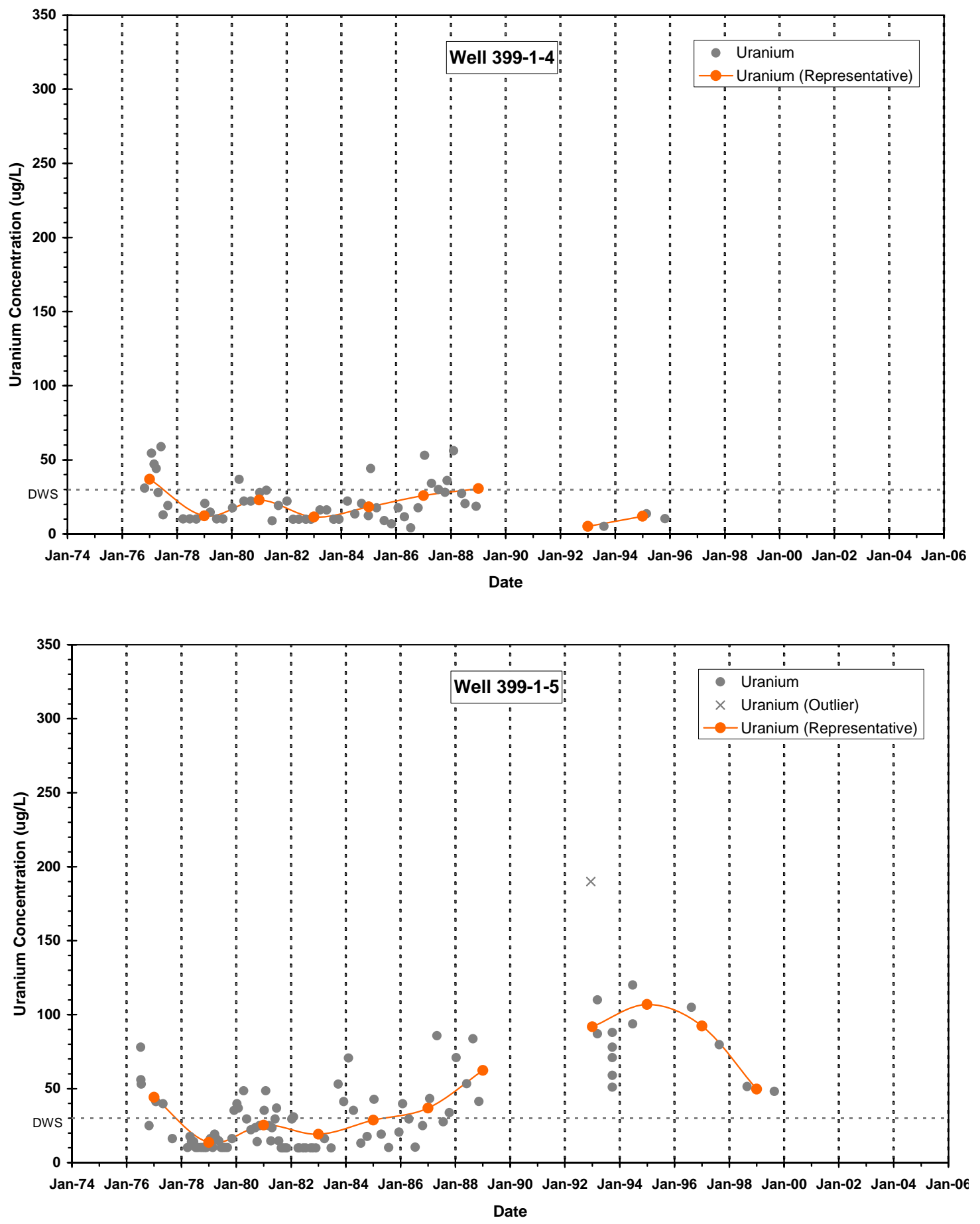


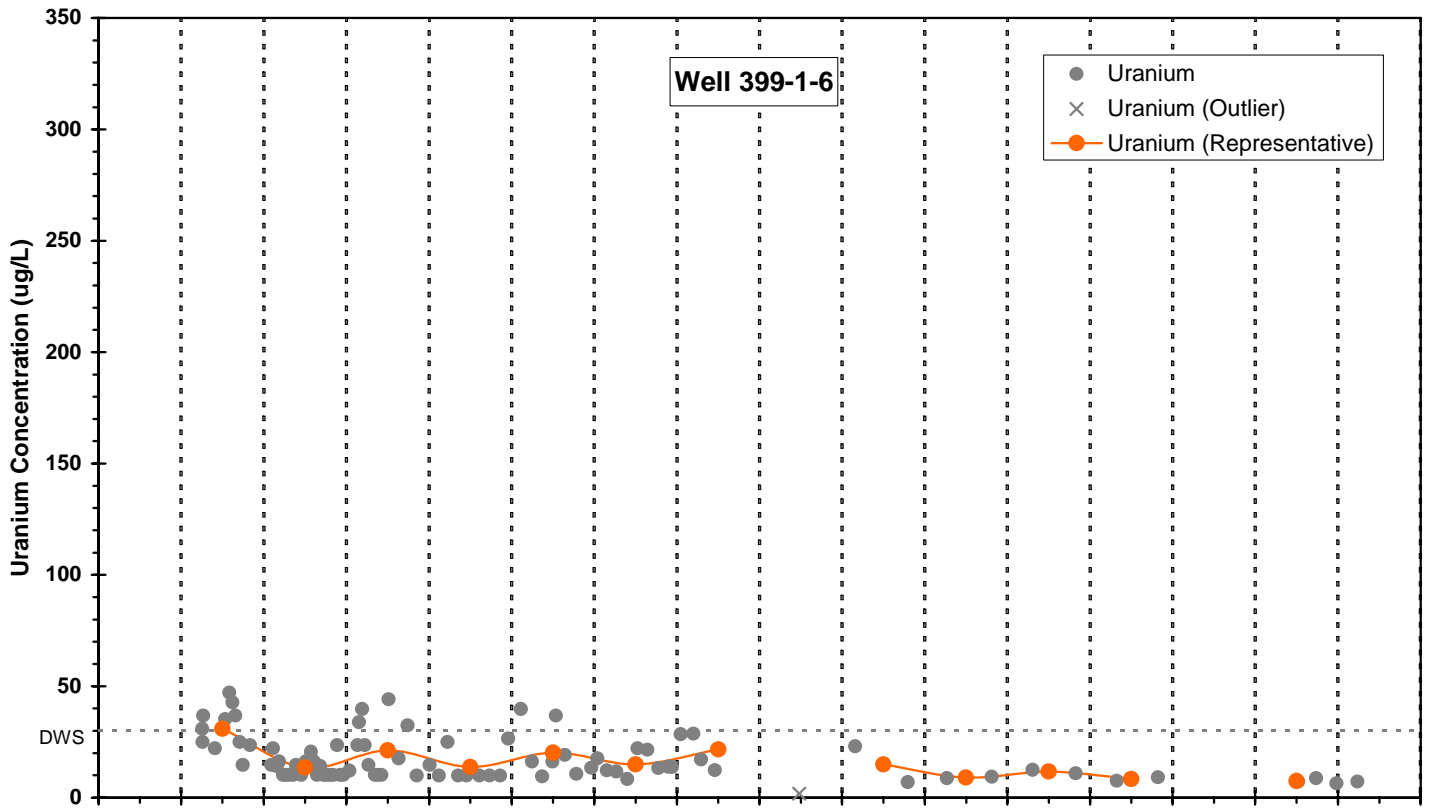

Jan-74 Jan-76 Jan-78 Jan-80 Jan-82 Jan-84 Jan-86 Jan-88 Jan-90 Jan-92 Jan-94 Jan-96 Jan-98 Jan-00 Jan-02 Jan-04 Jan-06 Date

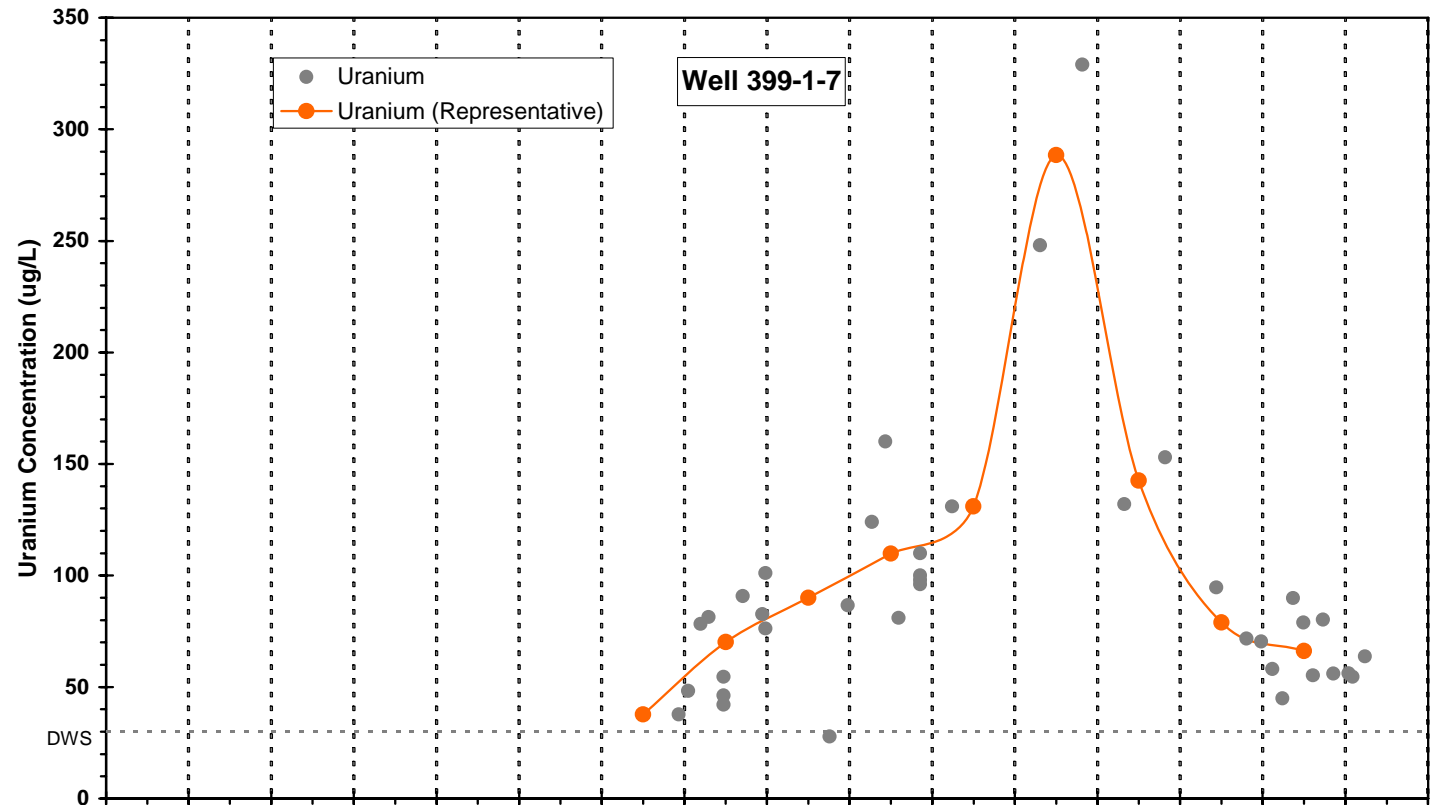

Jan-74 Jan-76 Jan-78 Jan-80 Jan-82 Jan-84 Jan-86 Jan-88 Jan-90 Jan-92 Jan-94 Jan-96 Jan-98 Jan-00 Jan-02 Jan-04 Jan-0 Date 

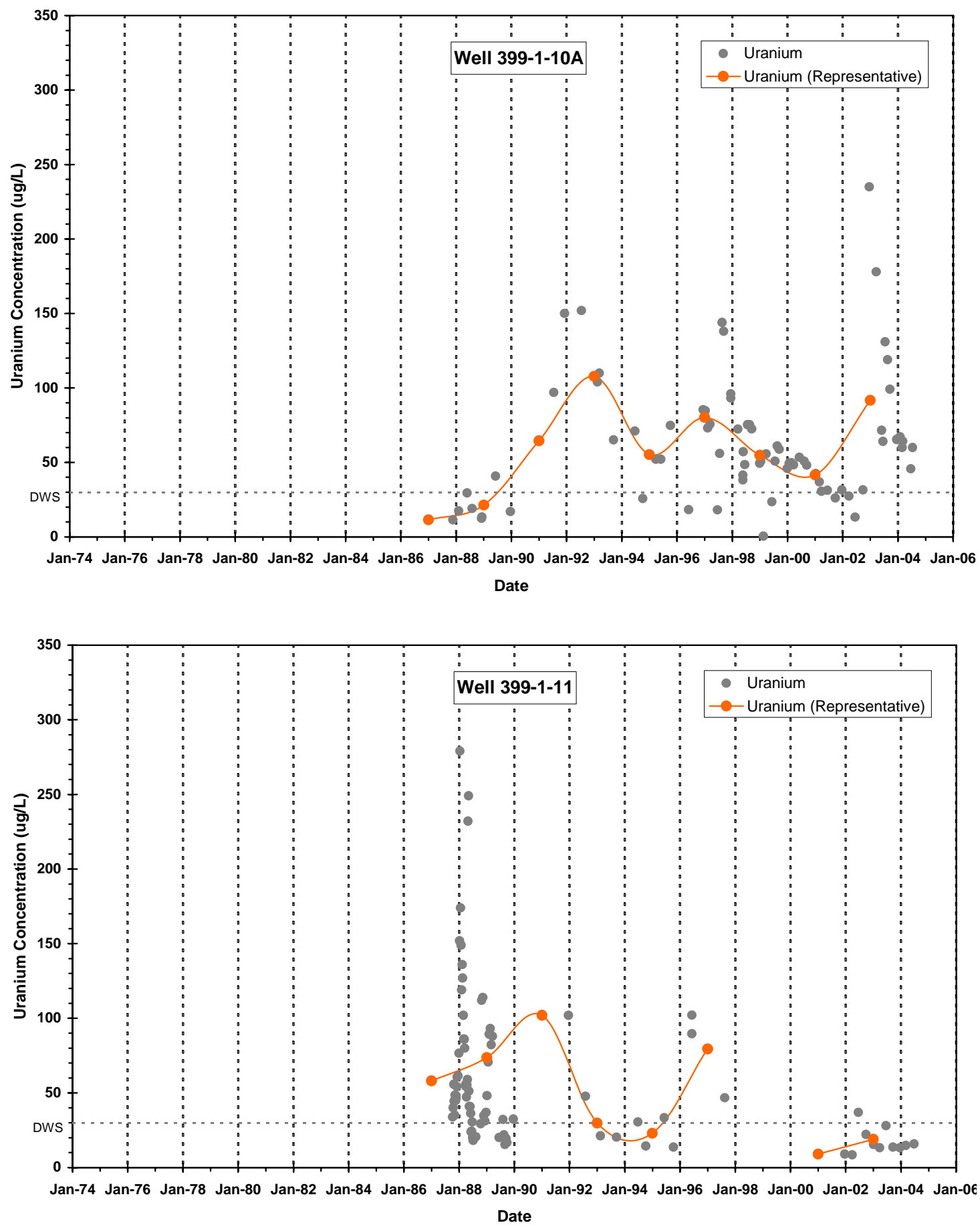

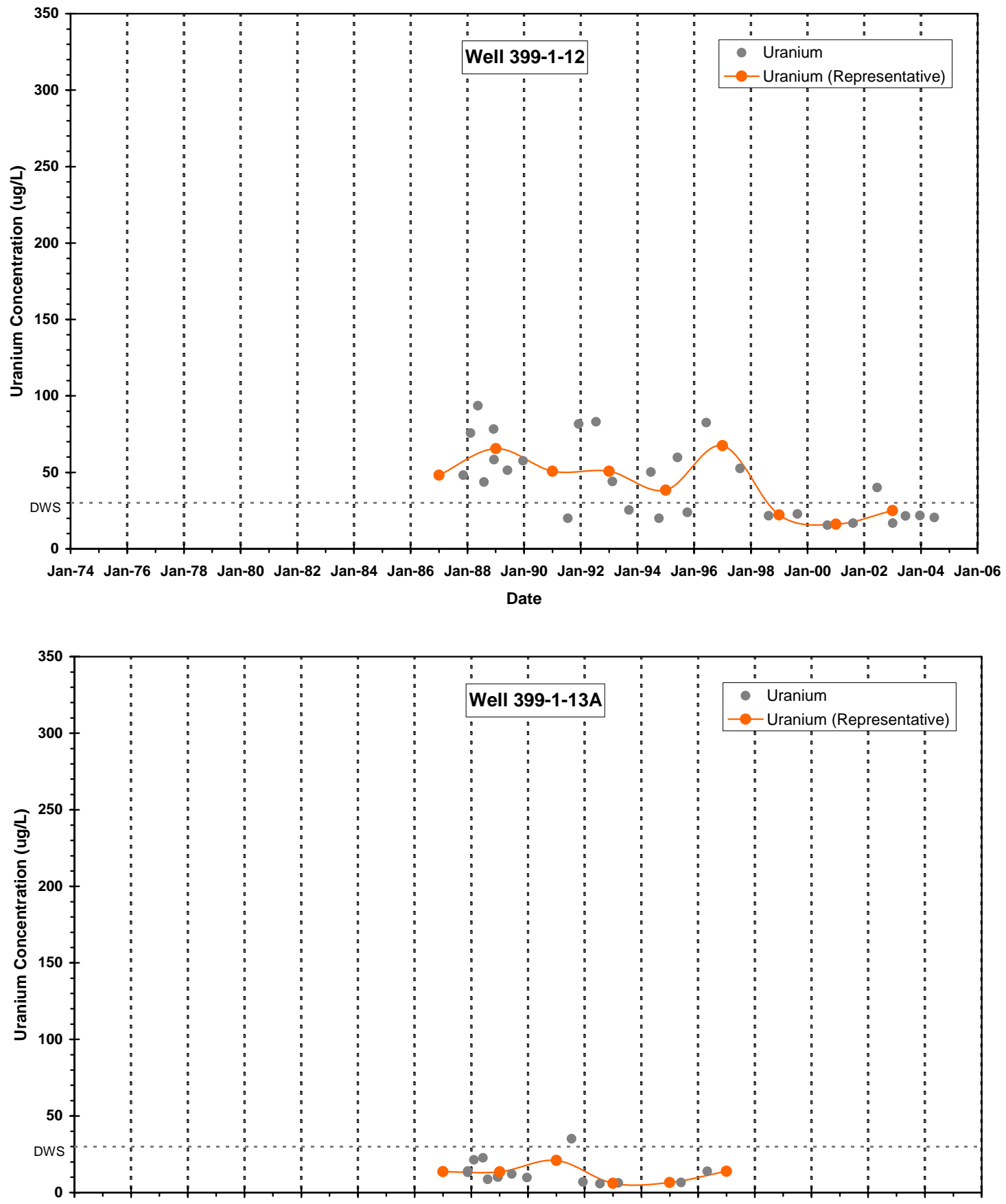

Jan-74 Jan-76 Jan-78 Jan-80 Jan-82 Jan-84 Jan-86 Jan-88 Jan-90 Jan-92 Jan-94 Jan-96 Jan-98 Jan-00 Jan-02 Jan-04 Jan-06 Date 


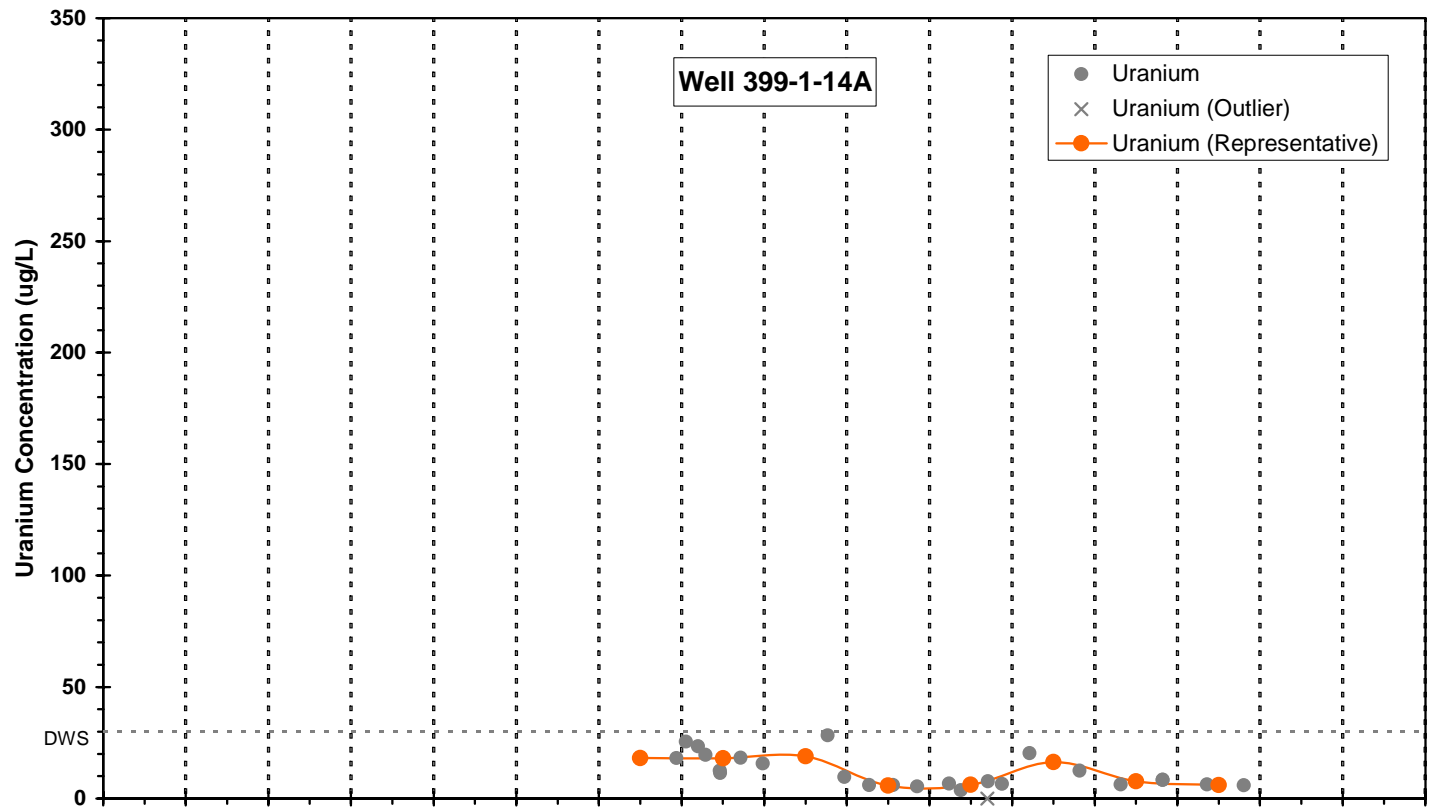

Jan-74 Jan-76 Jan-78 Jan-80 Jan-82 Jan-84 Jan-86 Jan-88 Jan-90 Jan-92 Jan-94 Jan-96 Jan-98 Jan-00 Jan-02 Jan-04 Jan-06 Date

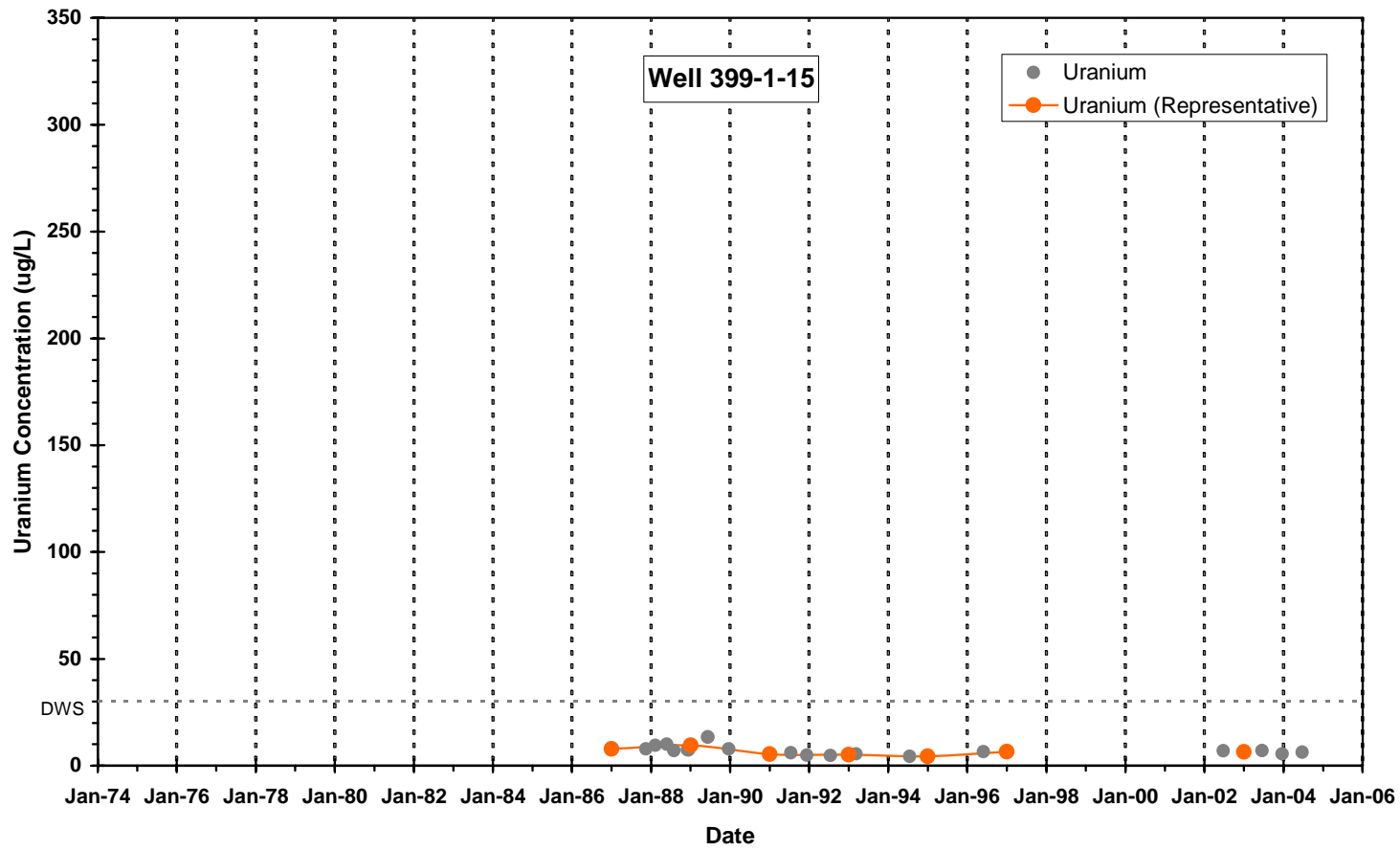




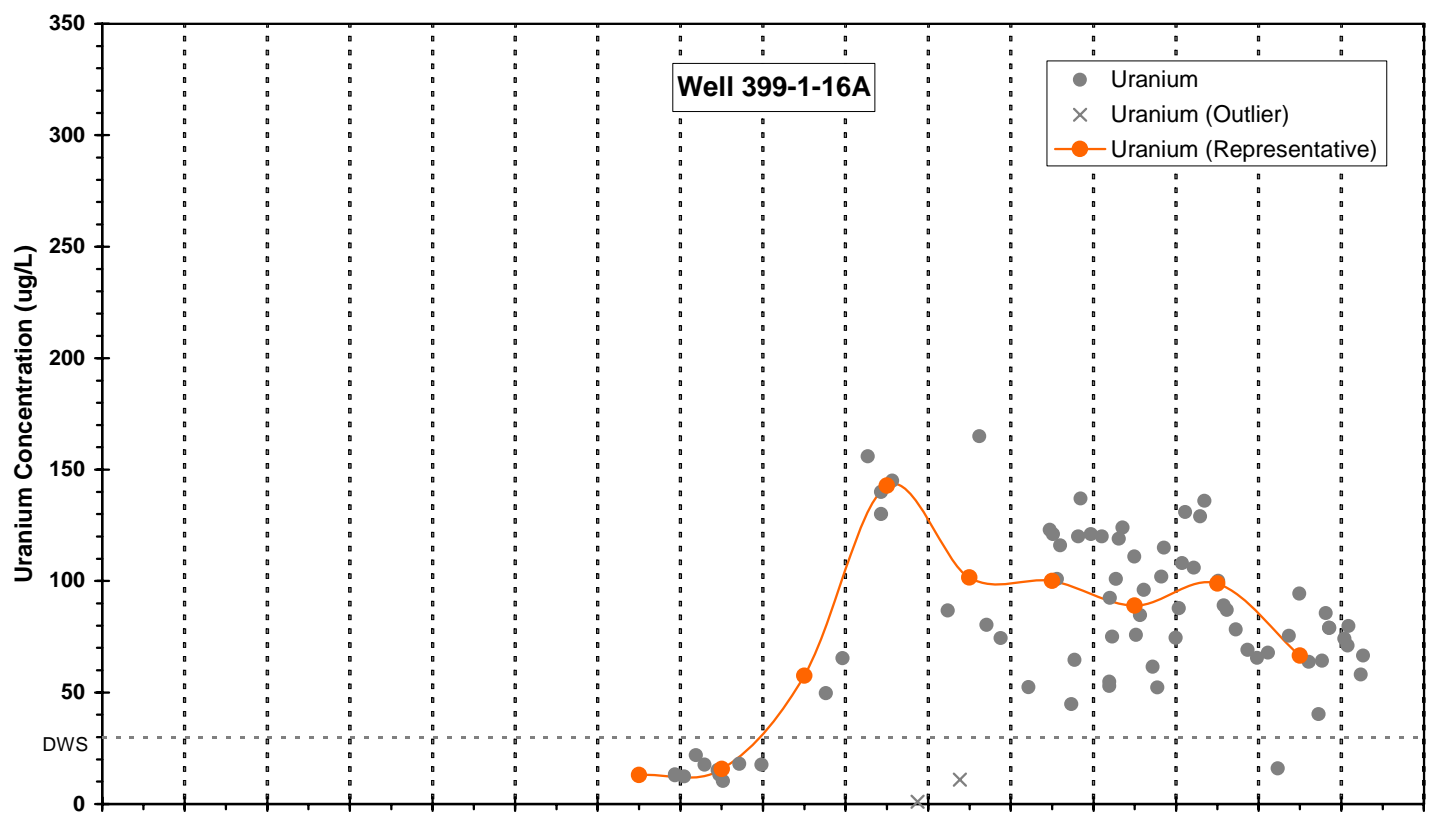

Jan-74 Jan-76 Jan-78 Jan-80 Jan-82 Jan-84 Jan-86 Jan-88 Jan-90 Jan-92 Jan-94 Jan-96 Jan-98 Jan-00 Jan-02 Jan-04 Jan-06 Date

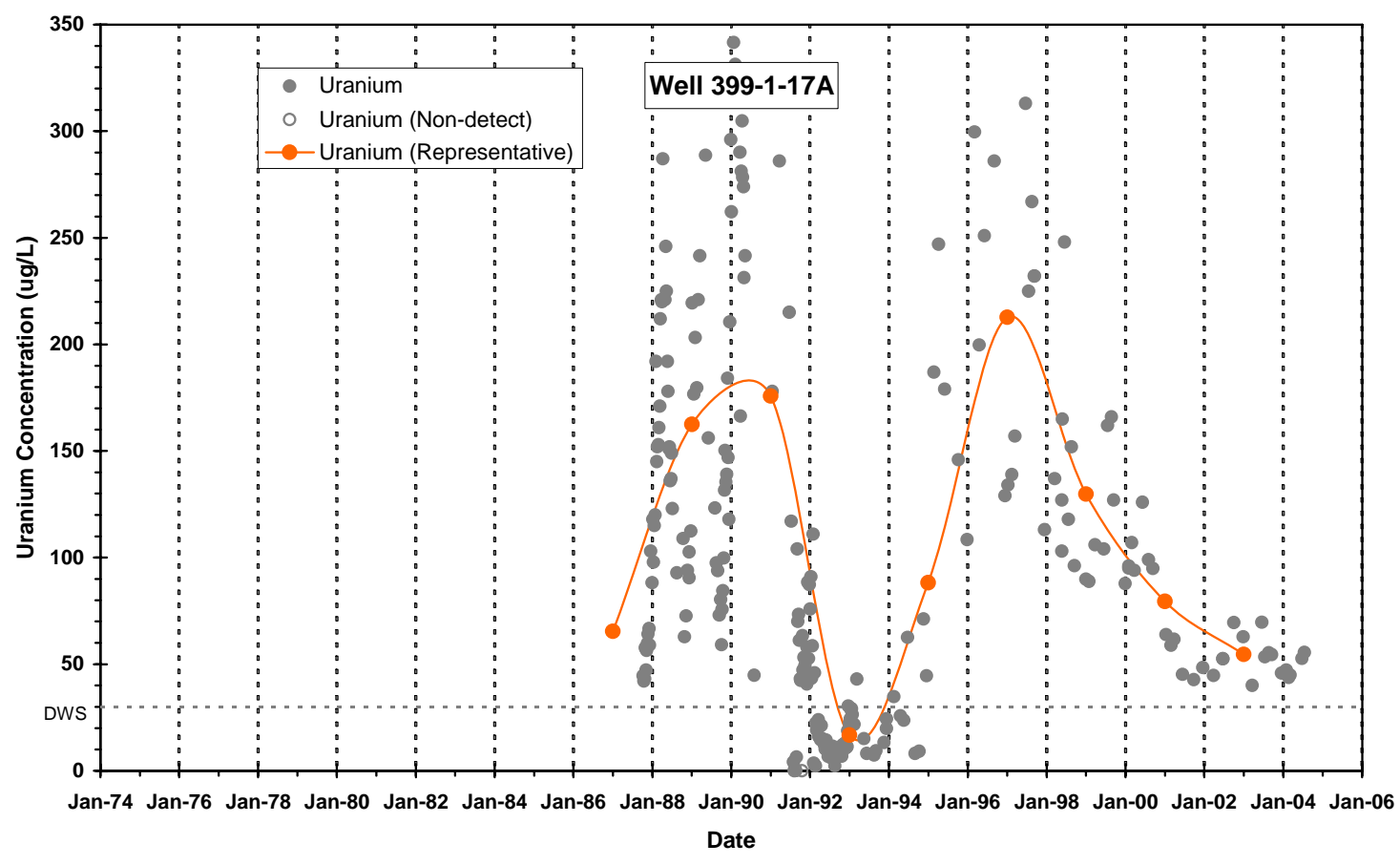



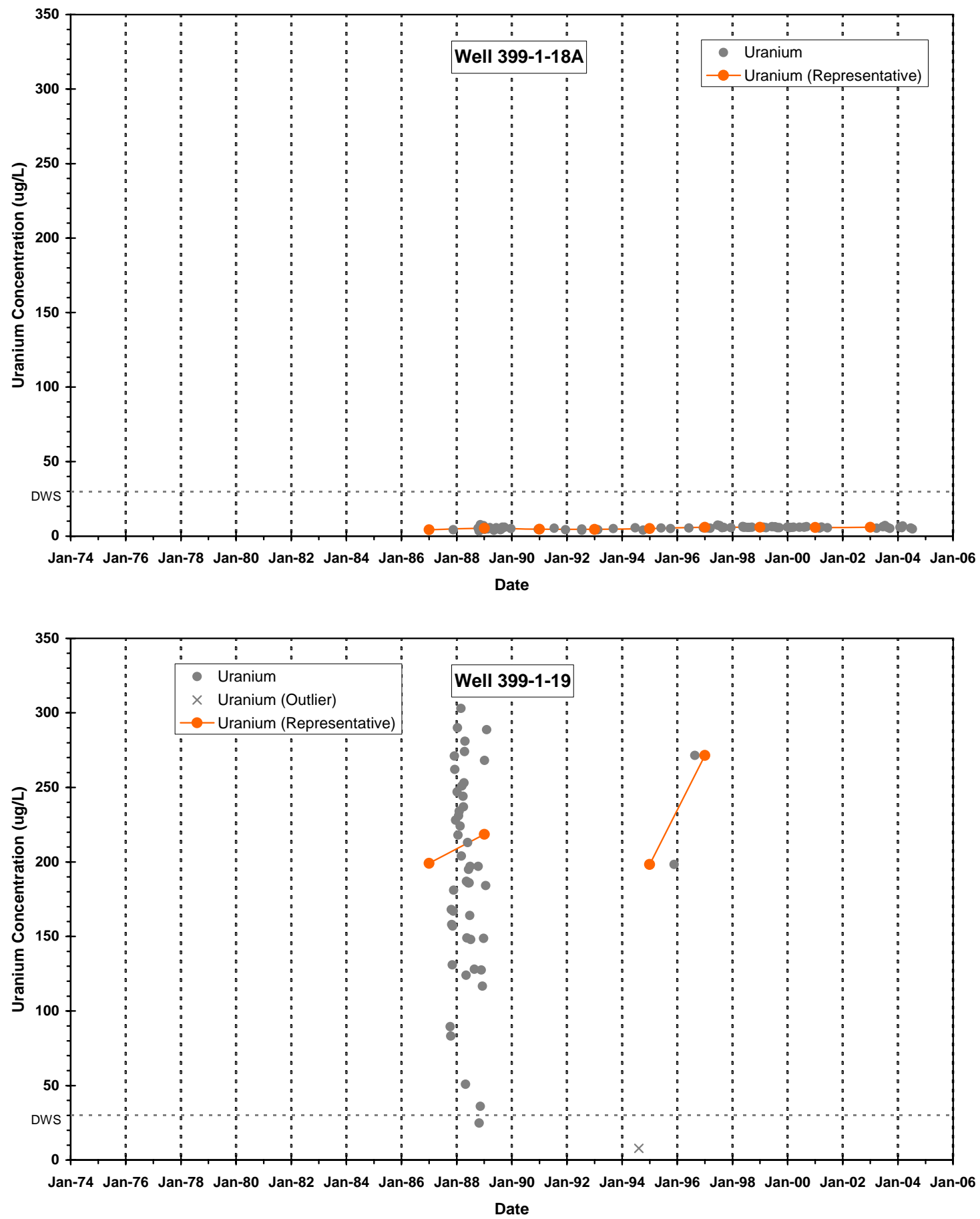

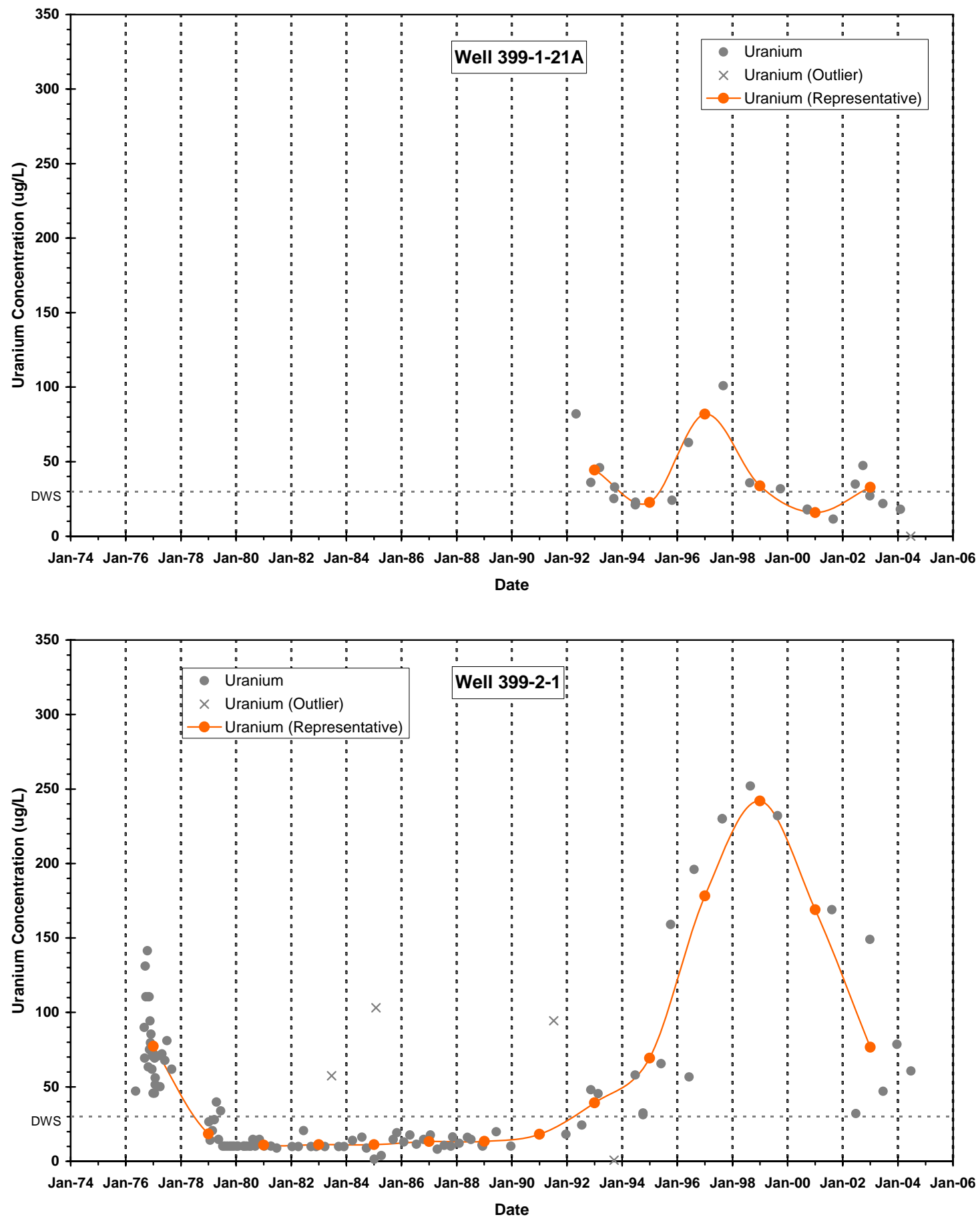


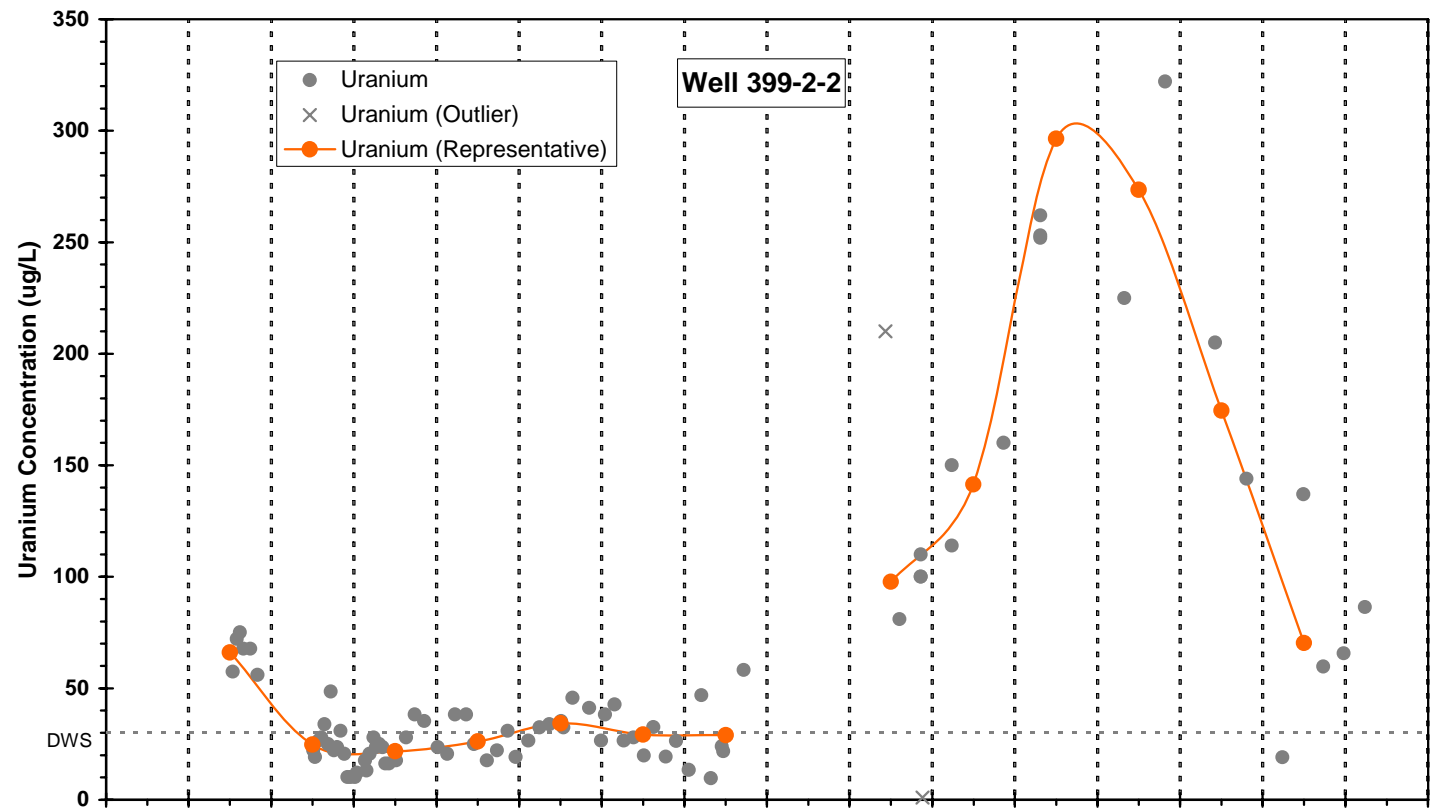

Jan-74 Jan-76 Jan-78 Jan-80 Jan-82 Jan-84 Jan-86 Jan-88 Jan-90 Jan-92 Jan-94 Jan-96 Jan-98 Jan-00 Jan-02 Jan-04 Jan-0 Date

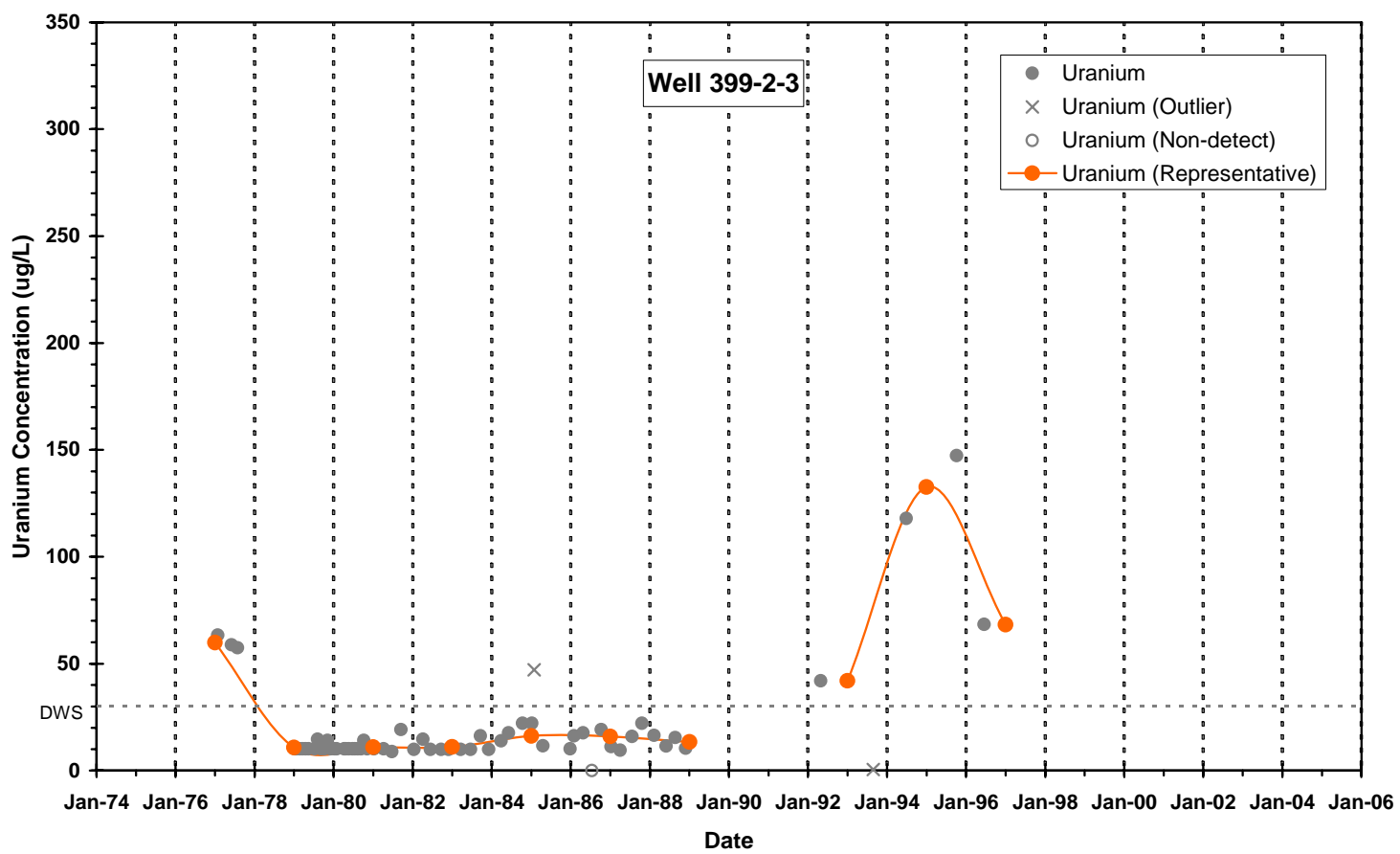



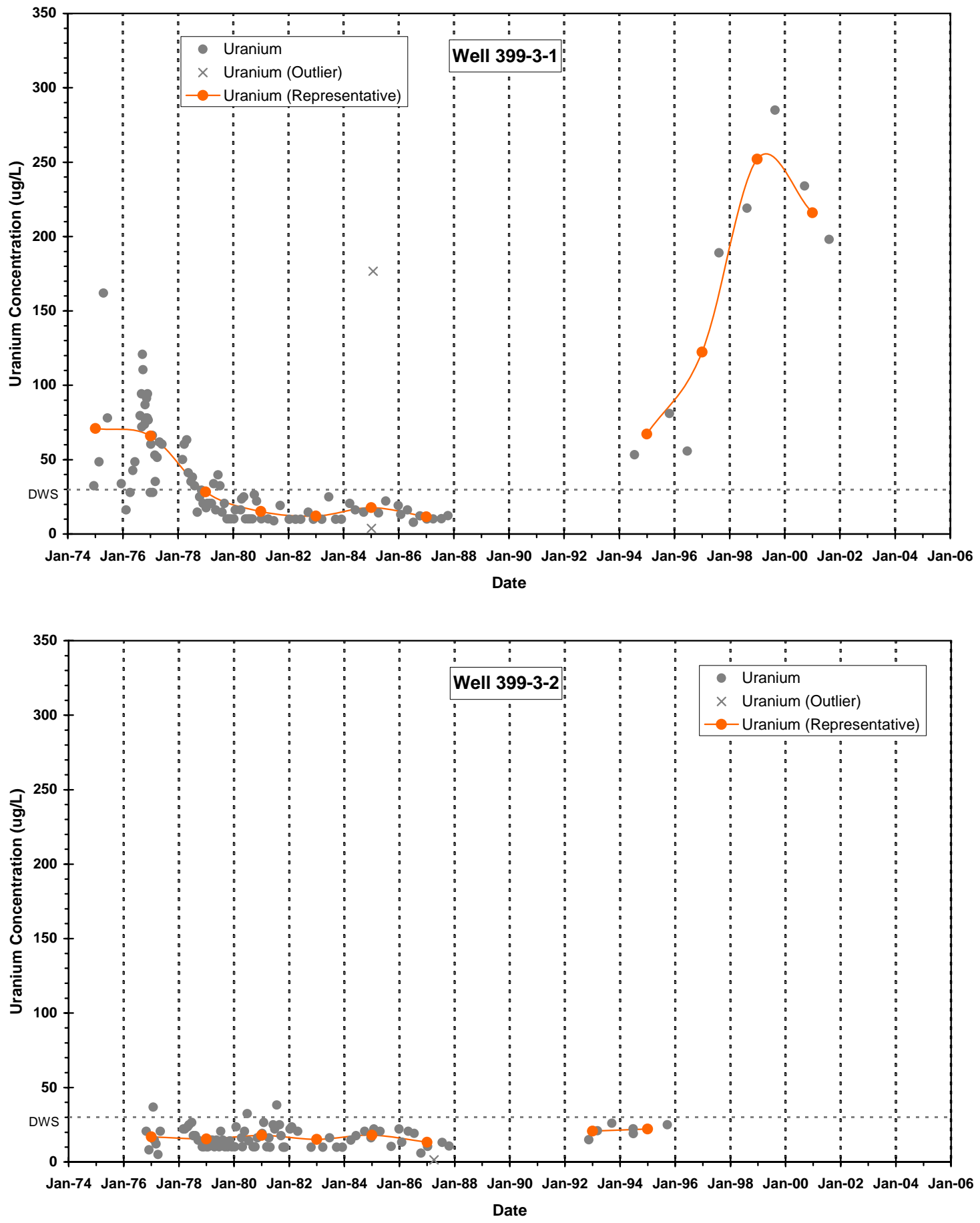

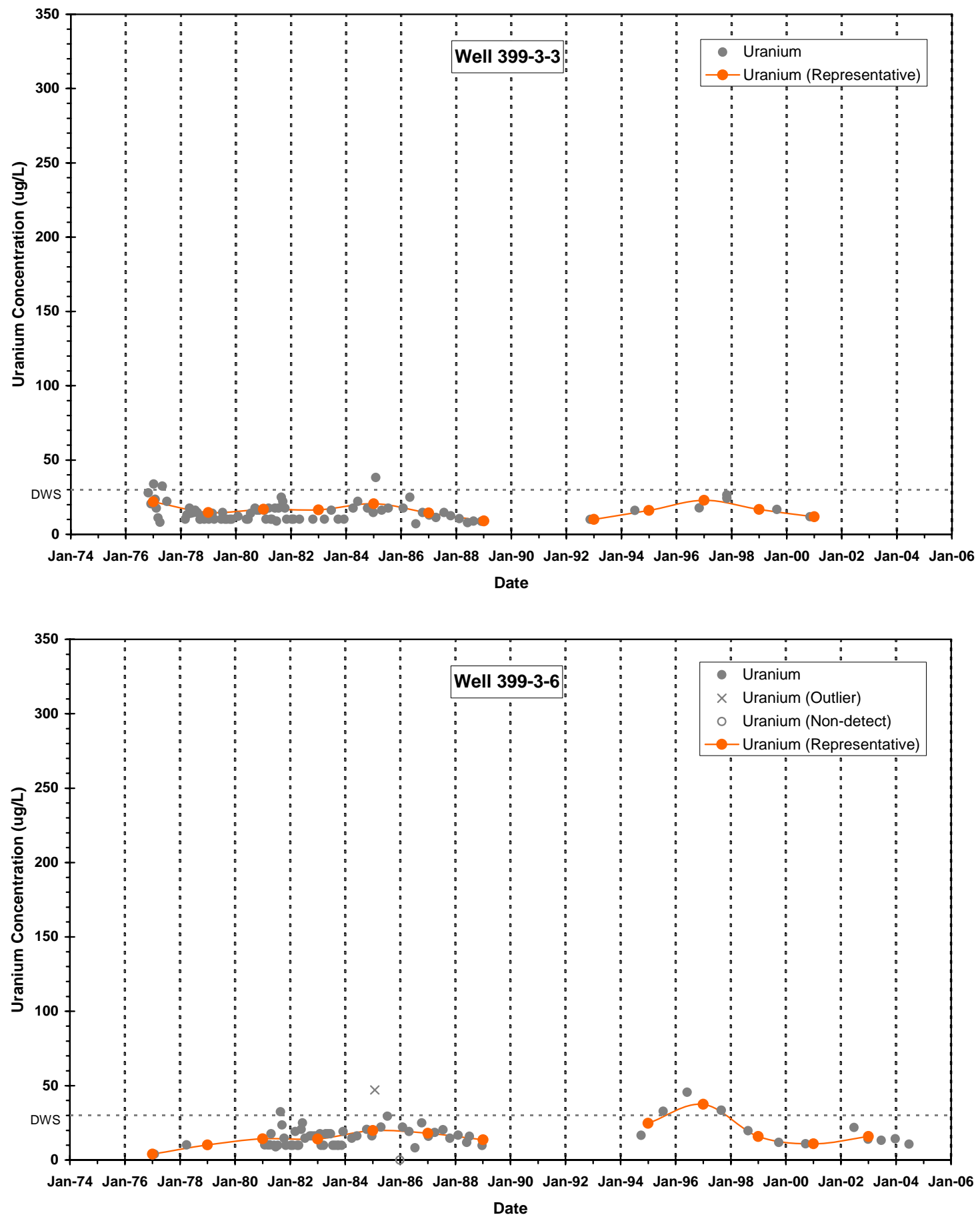

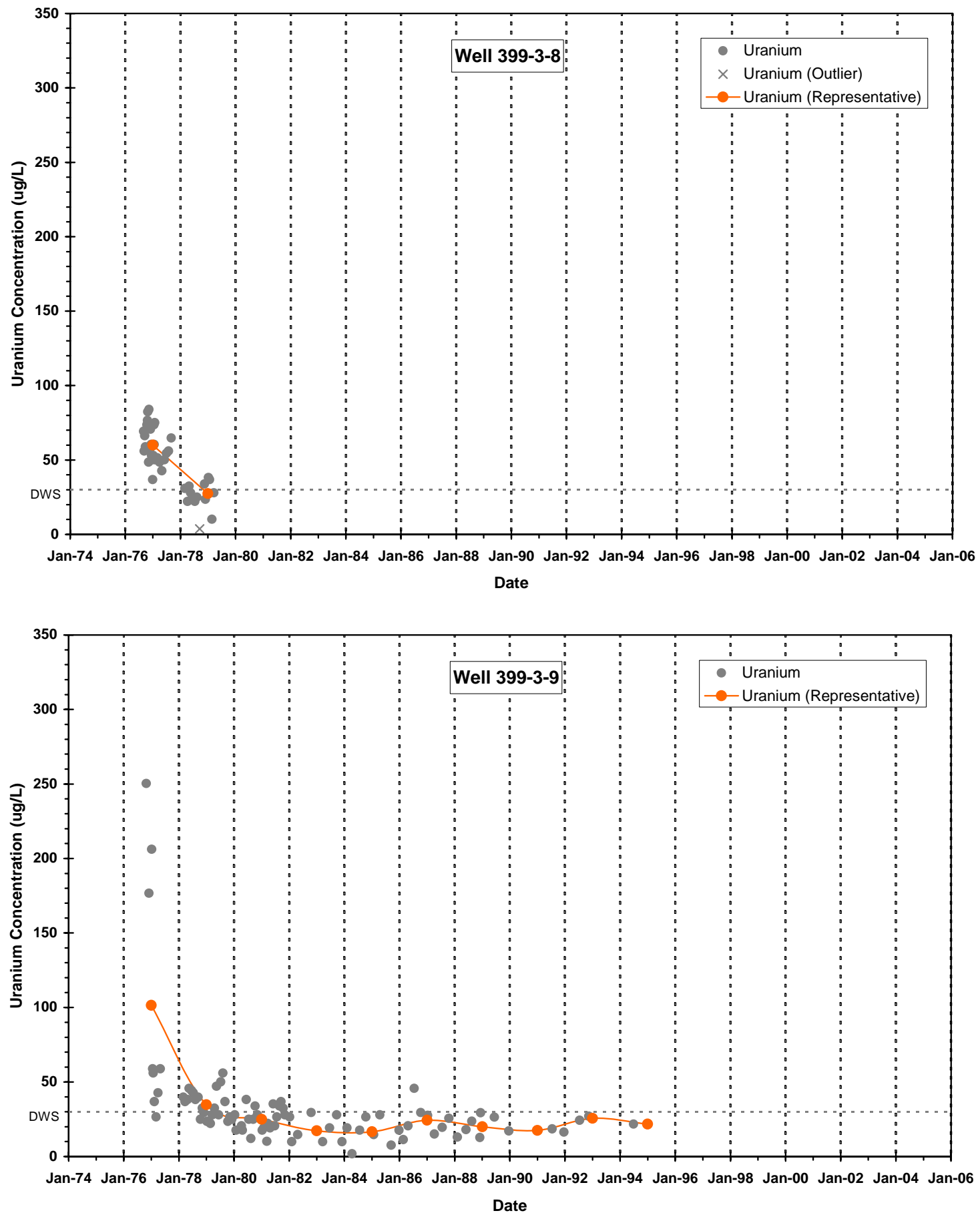


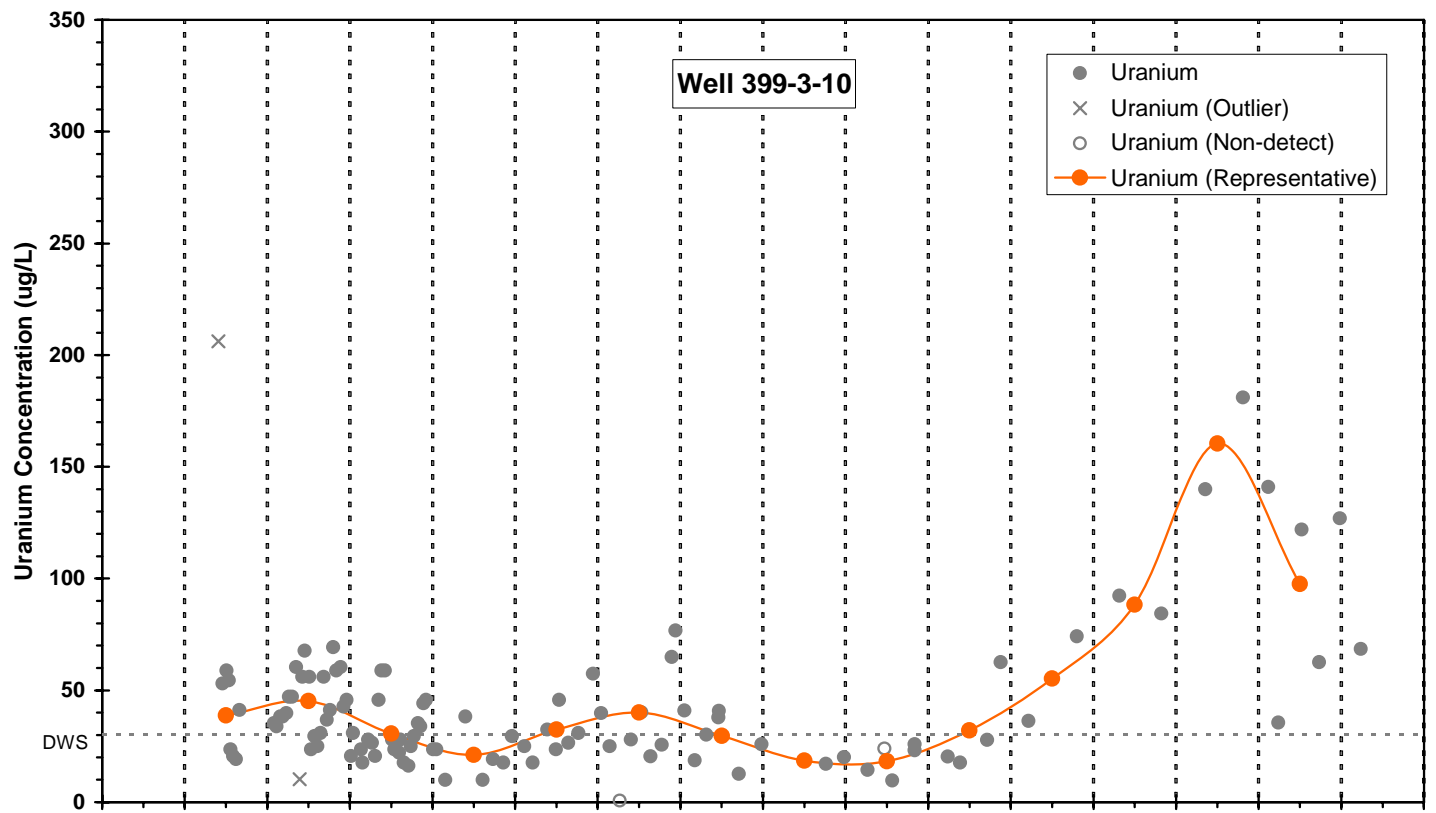

Jan-74 Jan-76 Jan-78 Jan-80 Jan-82 Jan-84 Jan-86 Jan-88 Jan-90 Jan-92 Jan-94 Jan-96 Jan-98 Jan-00 Jan-02 Jan-04 Jan-06 Date

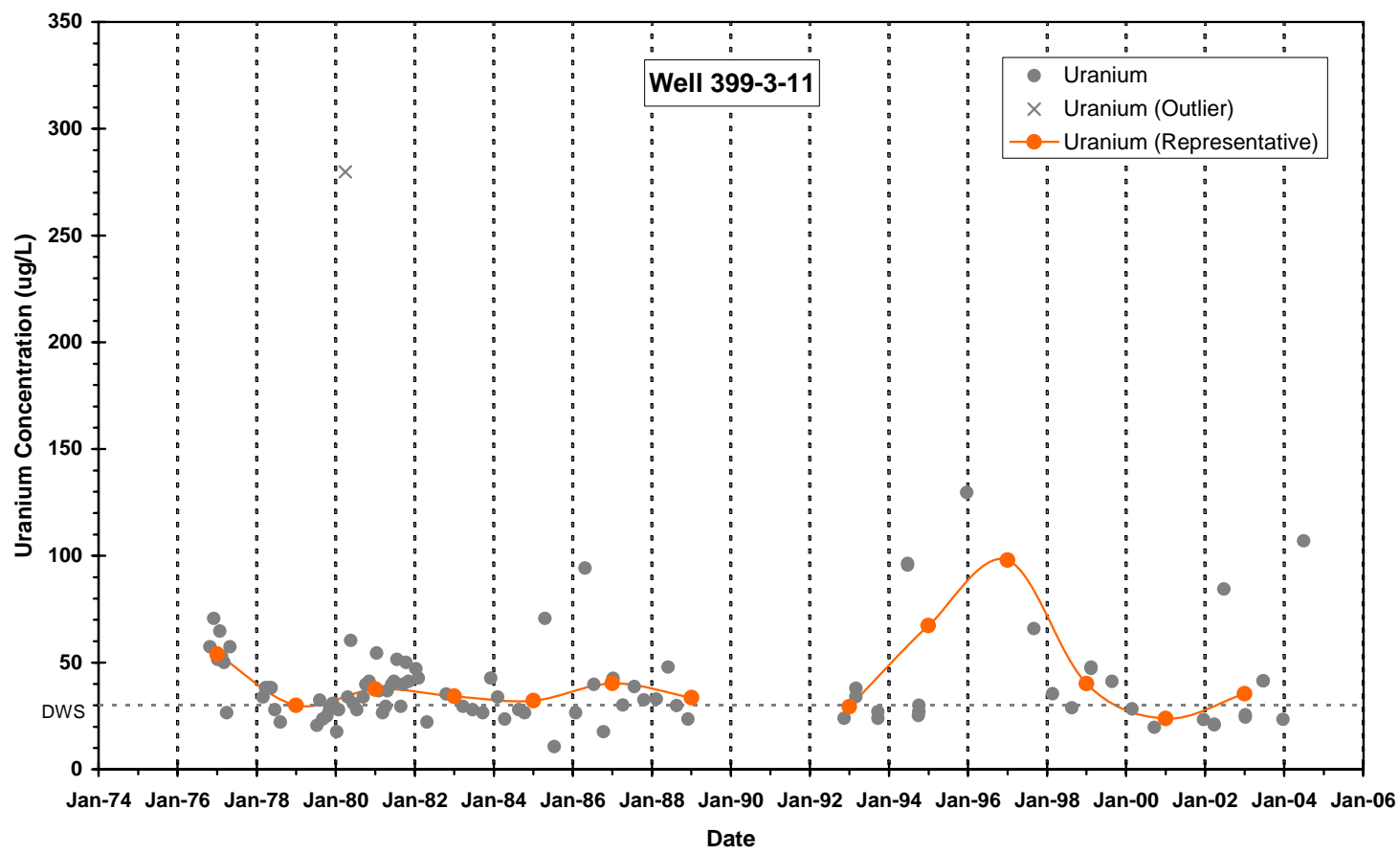



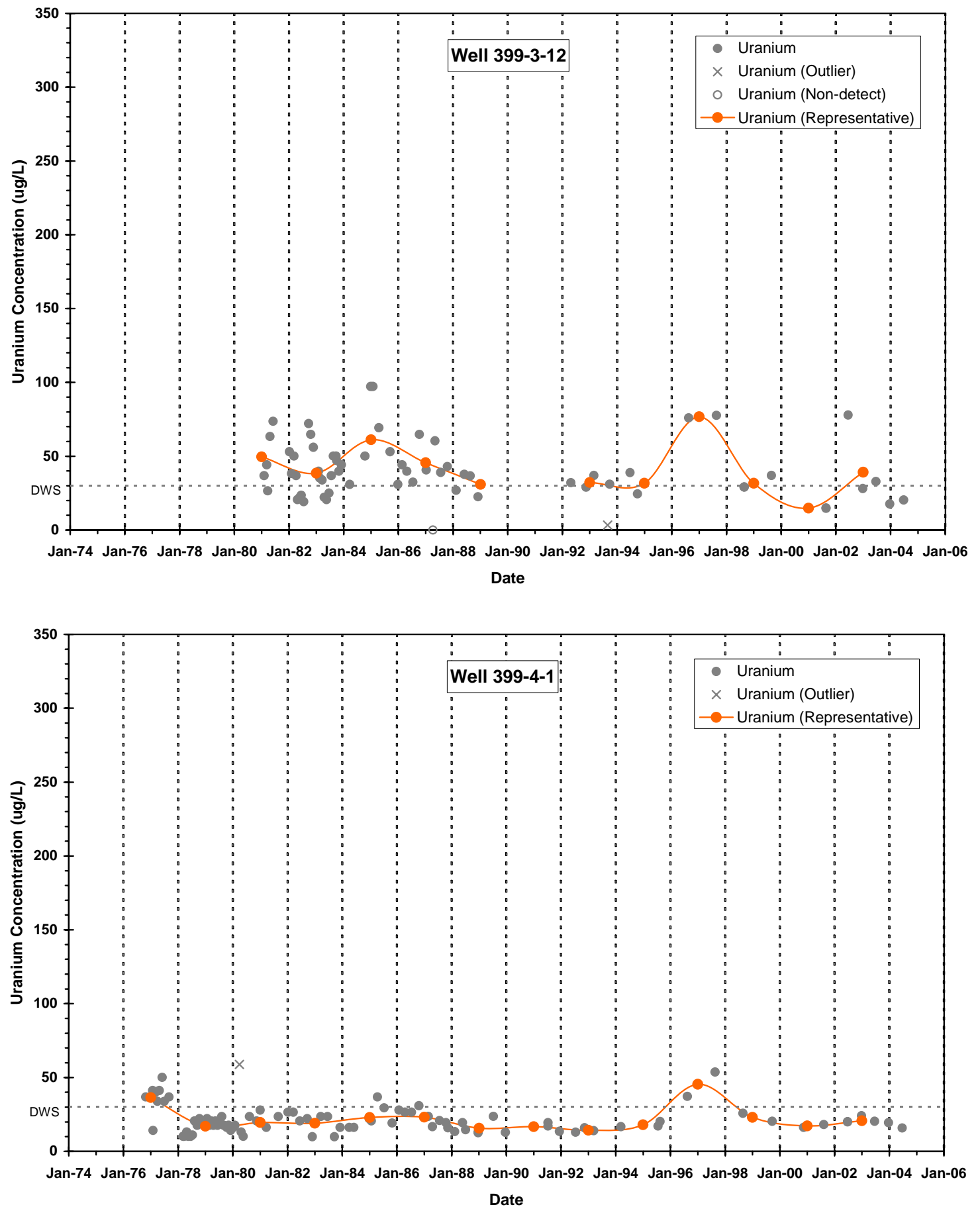

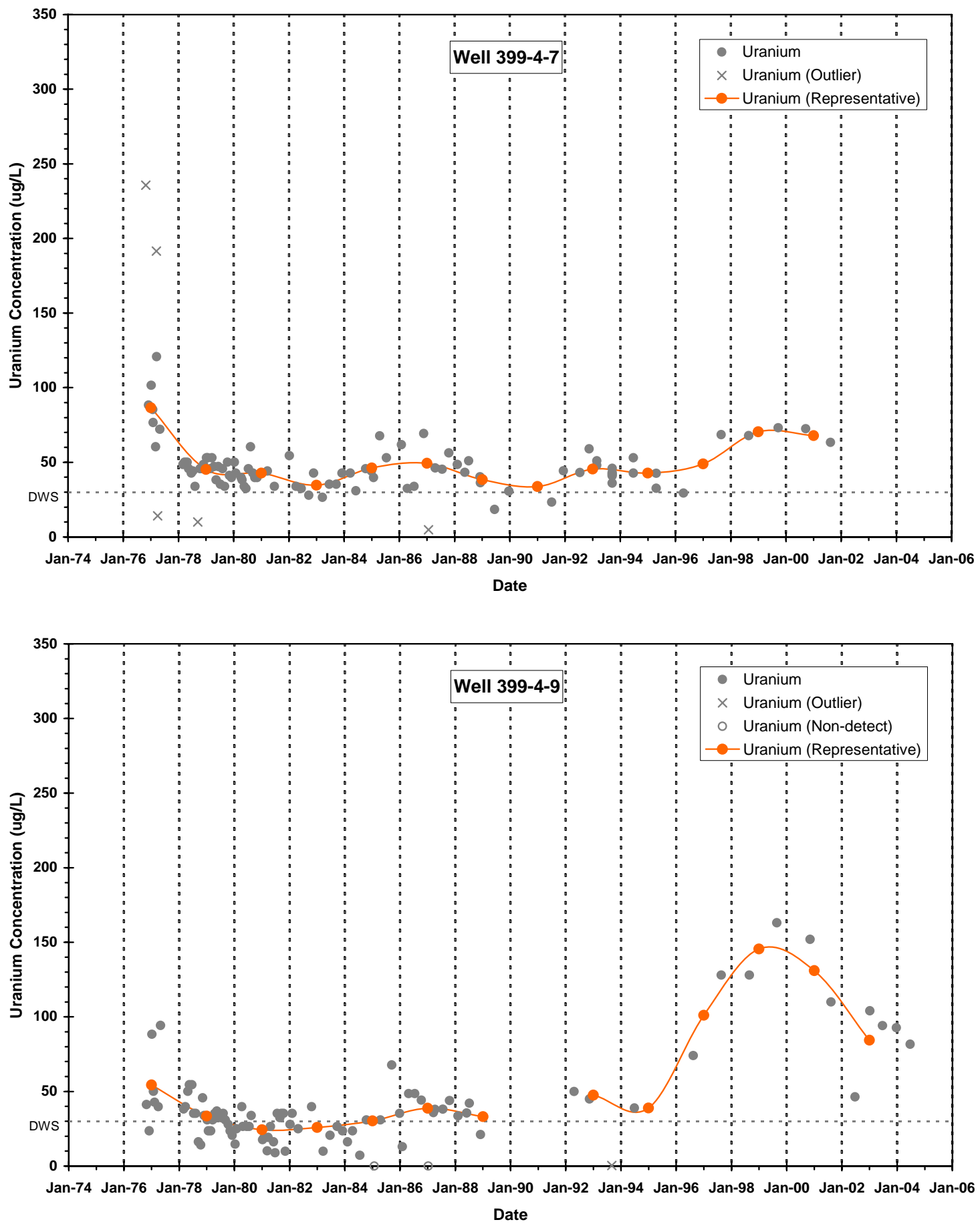

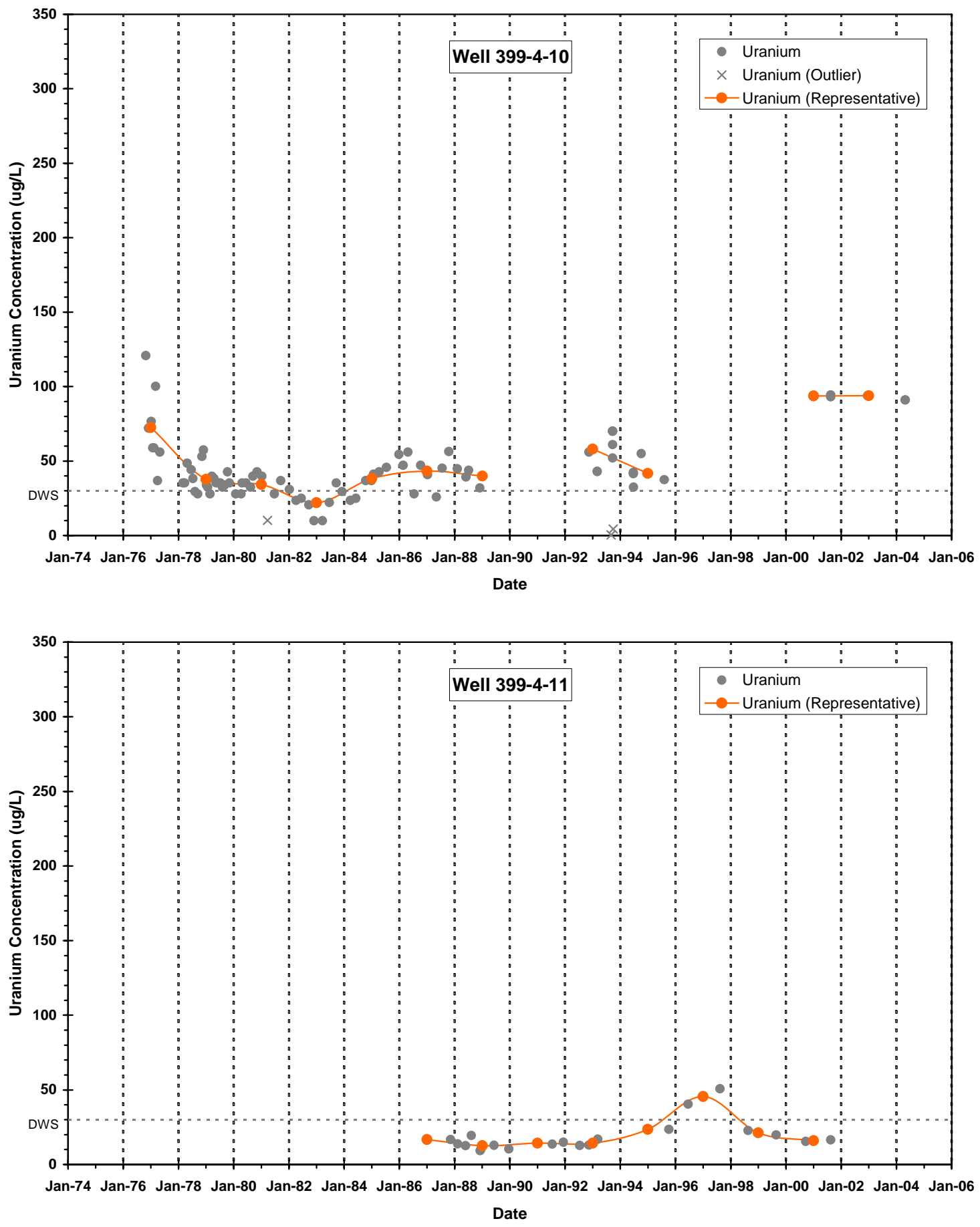

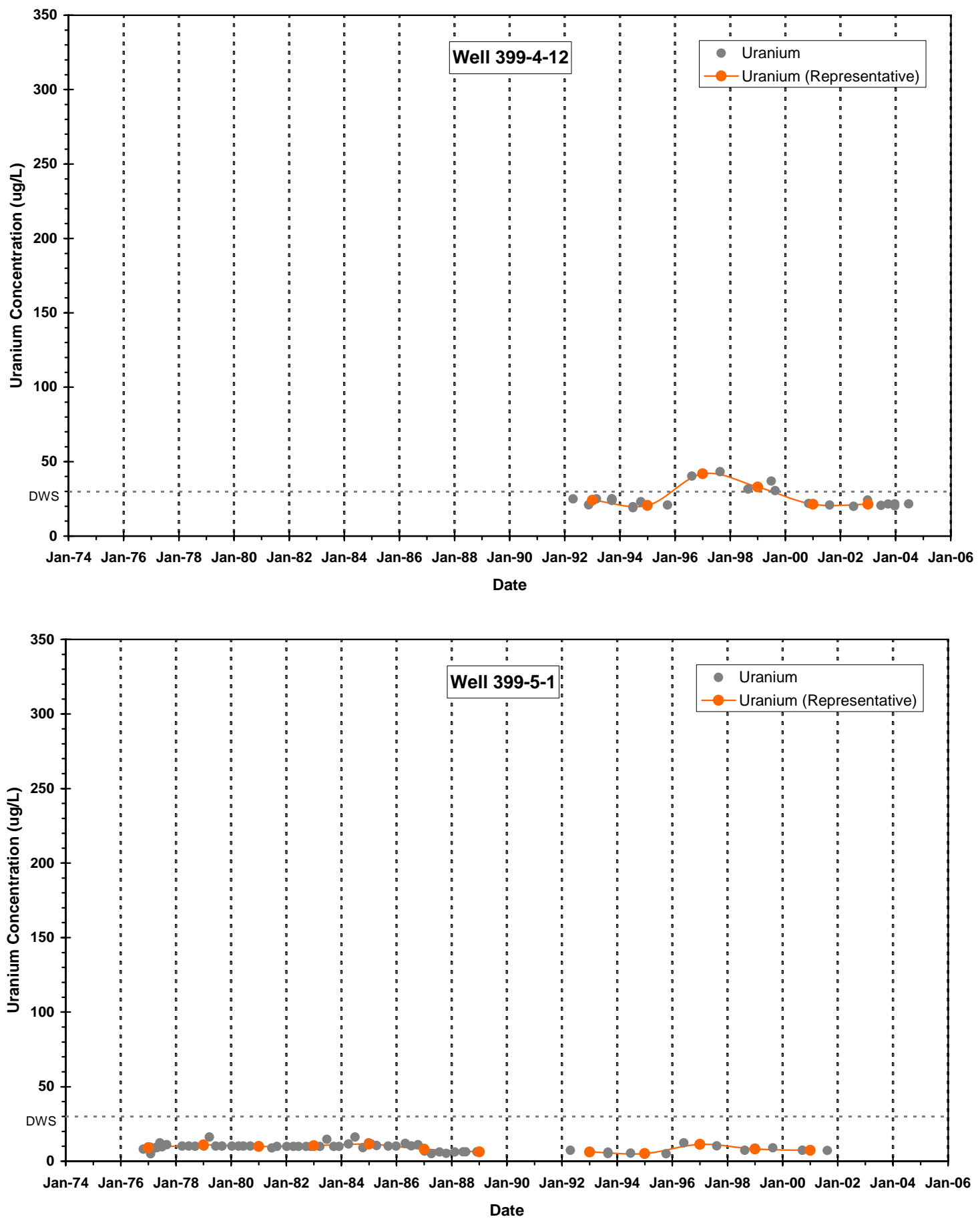


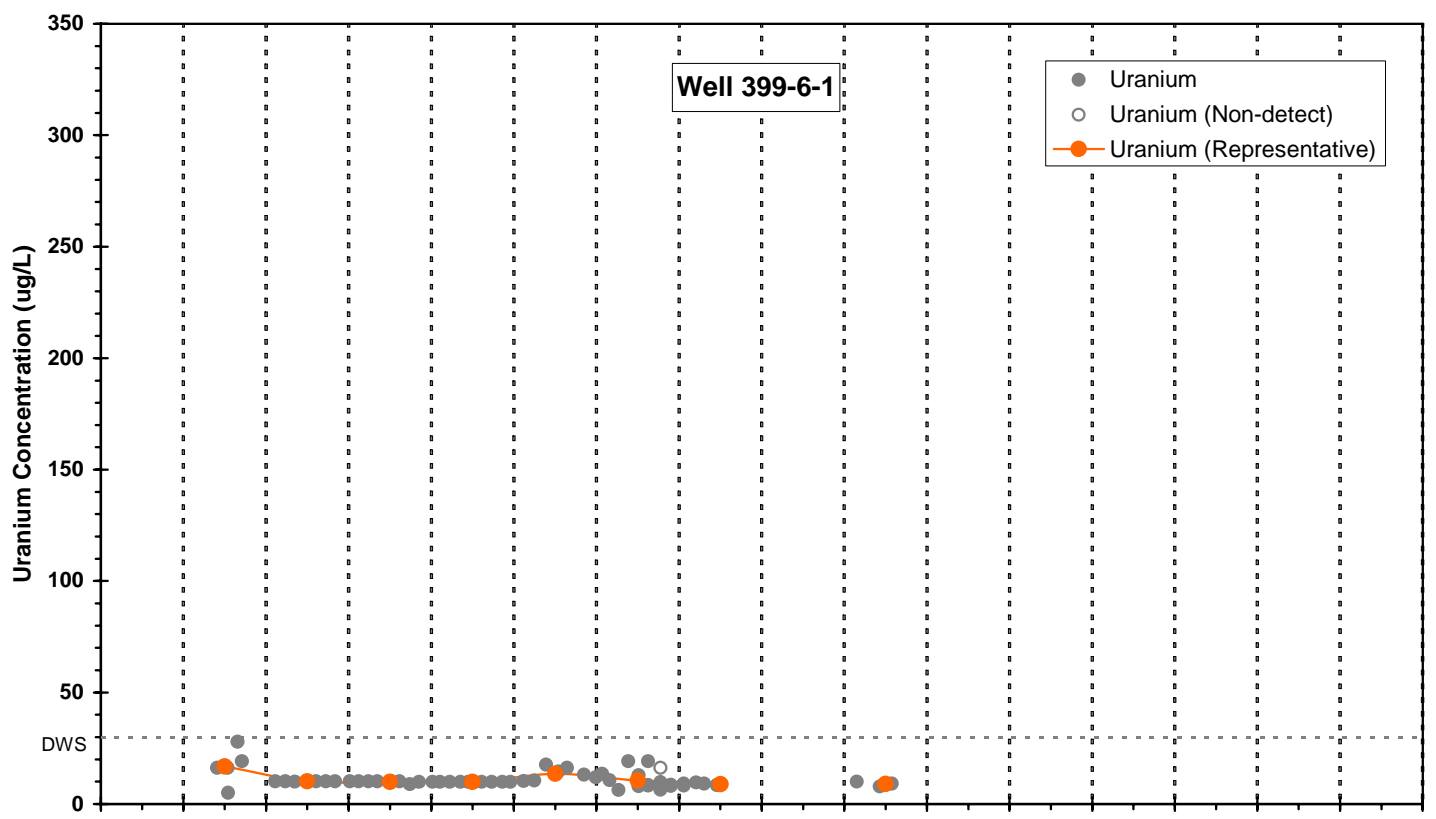

Jan-74 Jan-76 Jan-78 Jan-80 Jan-82 Jan-84 Jan-86 Jan-88 Jan-90 Jan-92 Jan-94 Jan-96 Jan-98 Jan-00 Jan-02 Jan-04 Jan-06 Date

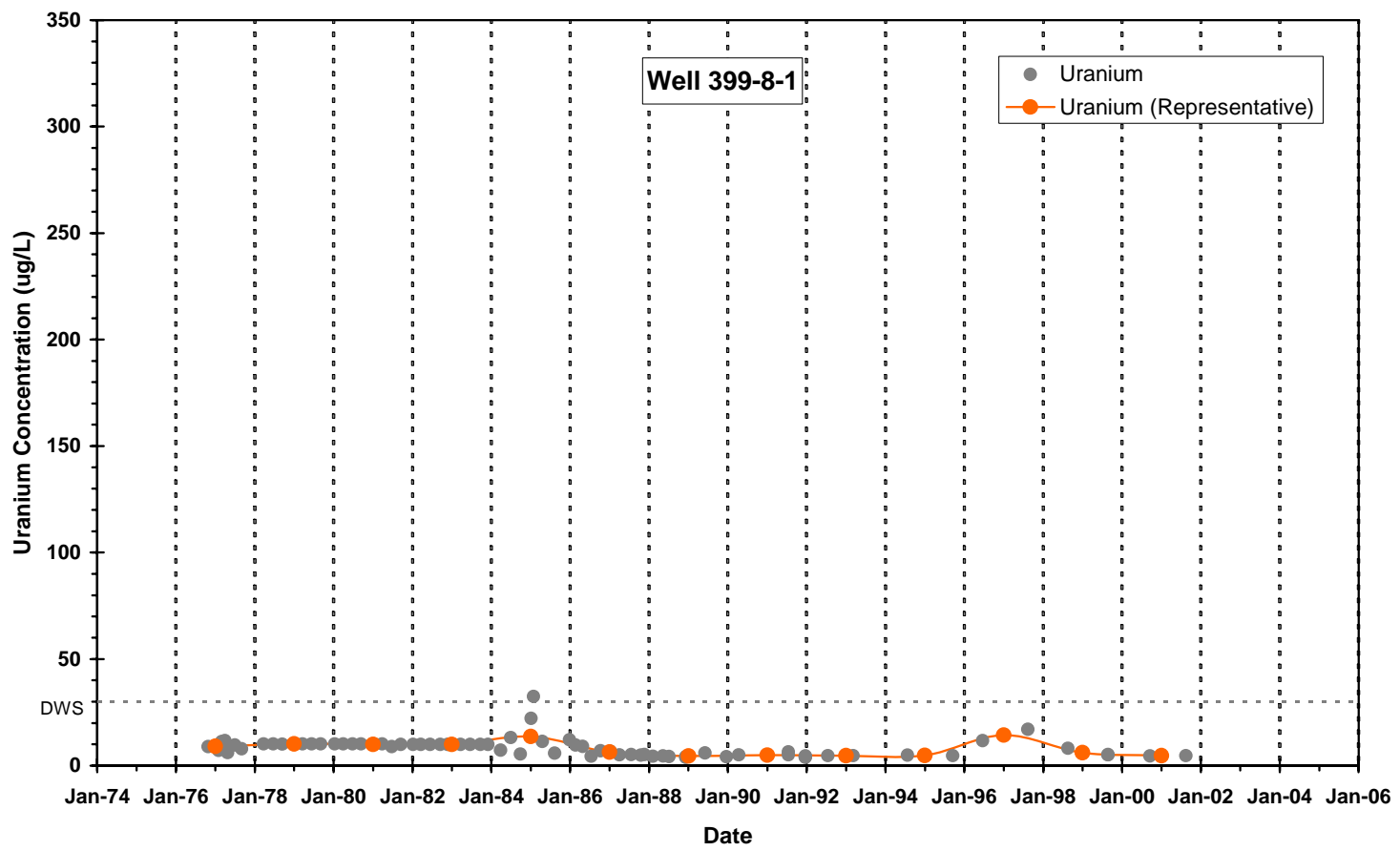




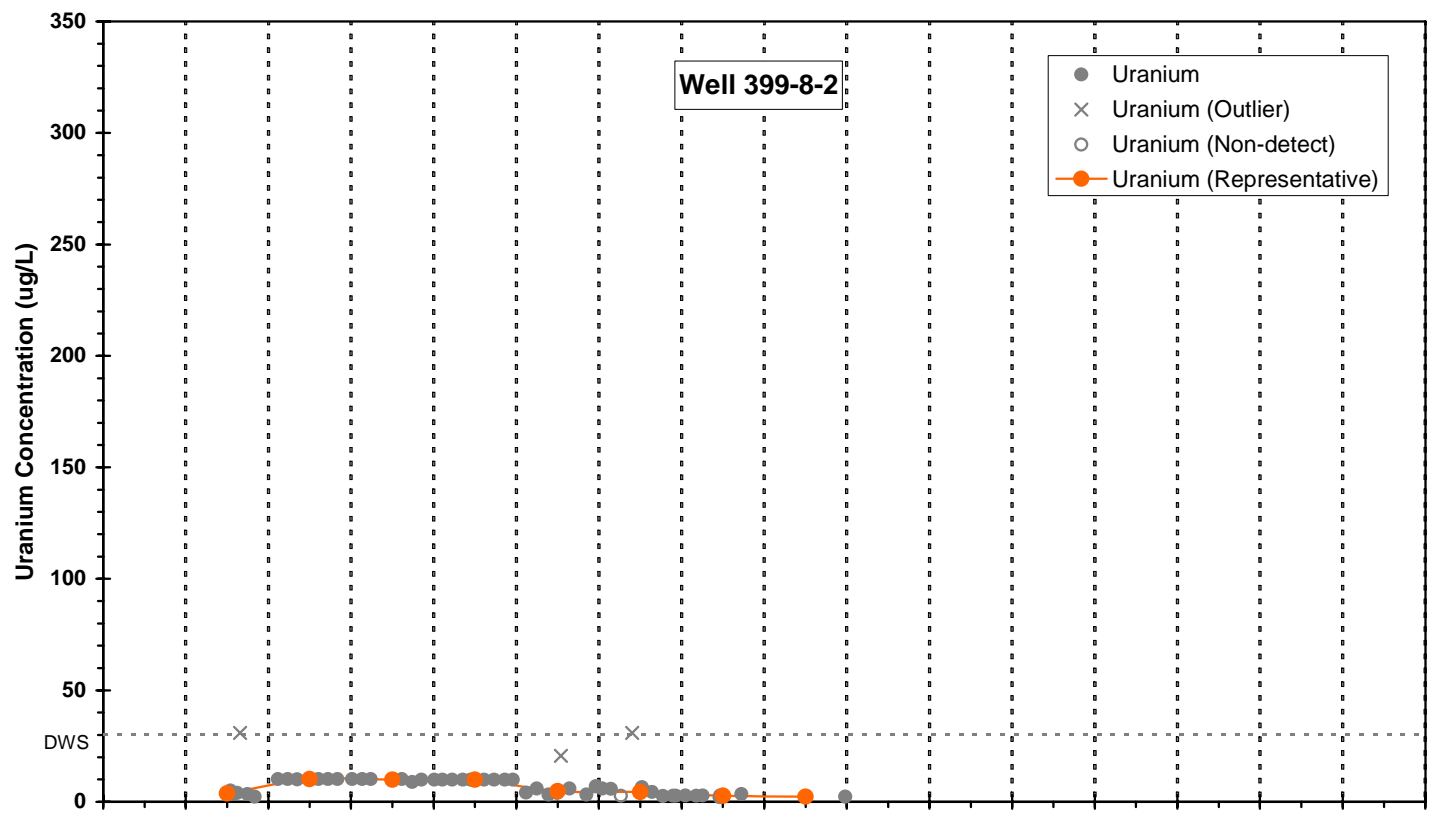

Jan-74 Jan-76 Jan-78 Jan-80 Jan-82 Jan-84 Jan-86 Jan-88 Jan-90 Jan-92 Jan-94 Jan-96 Jan-98 Jan-00 Jan-02 Jan-04 Jan-06 Date

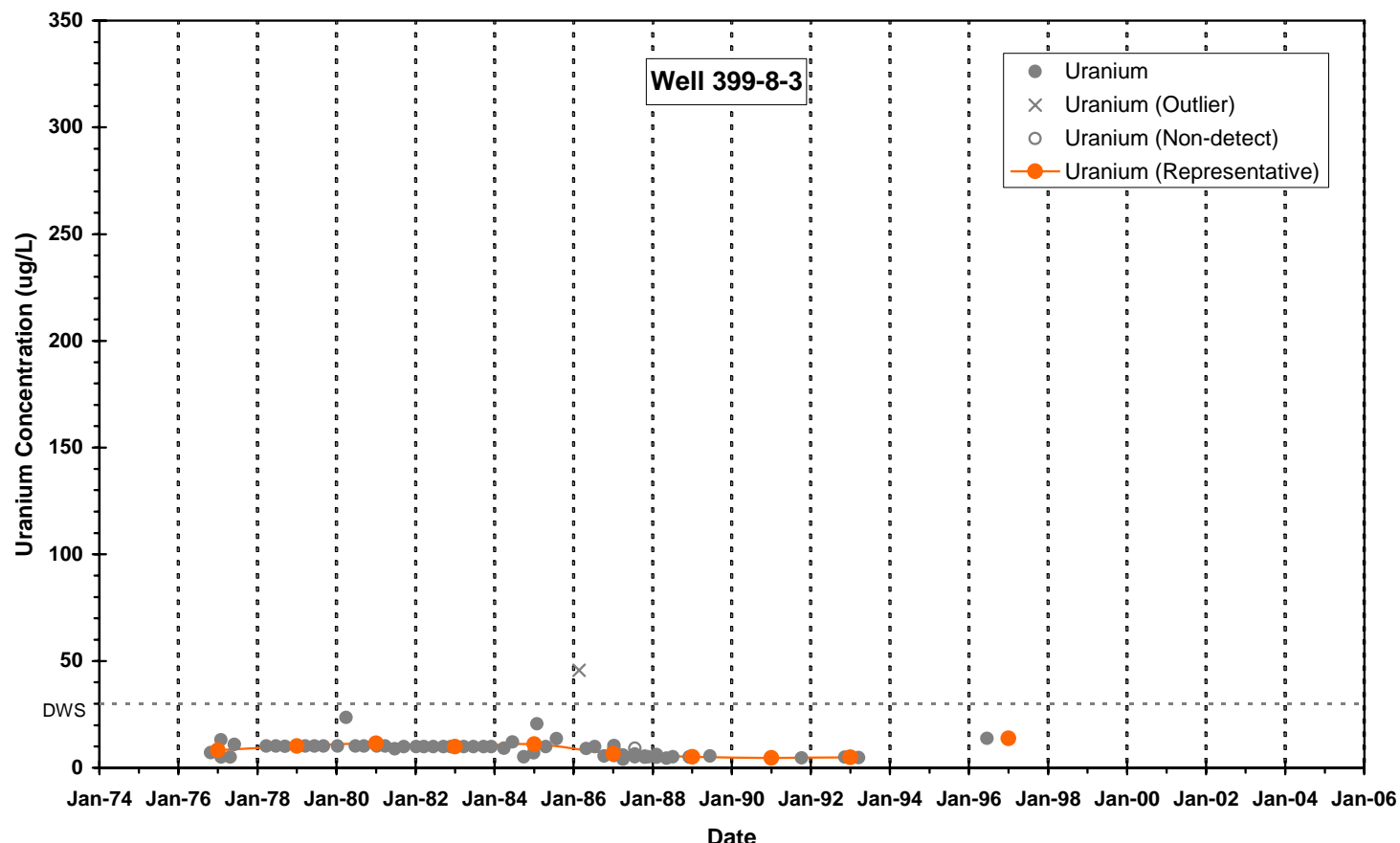



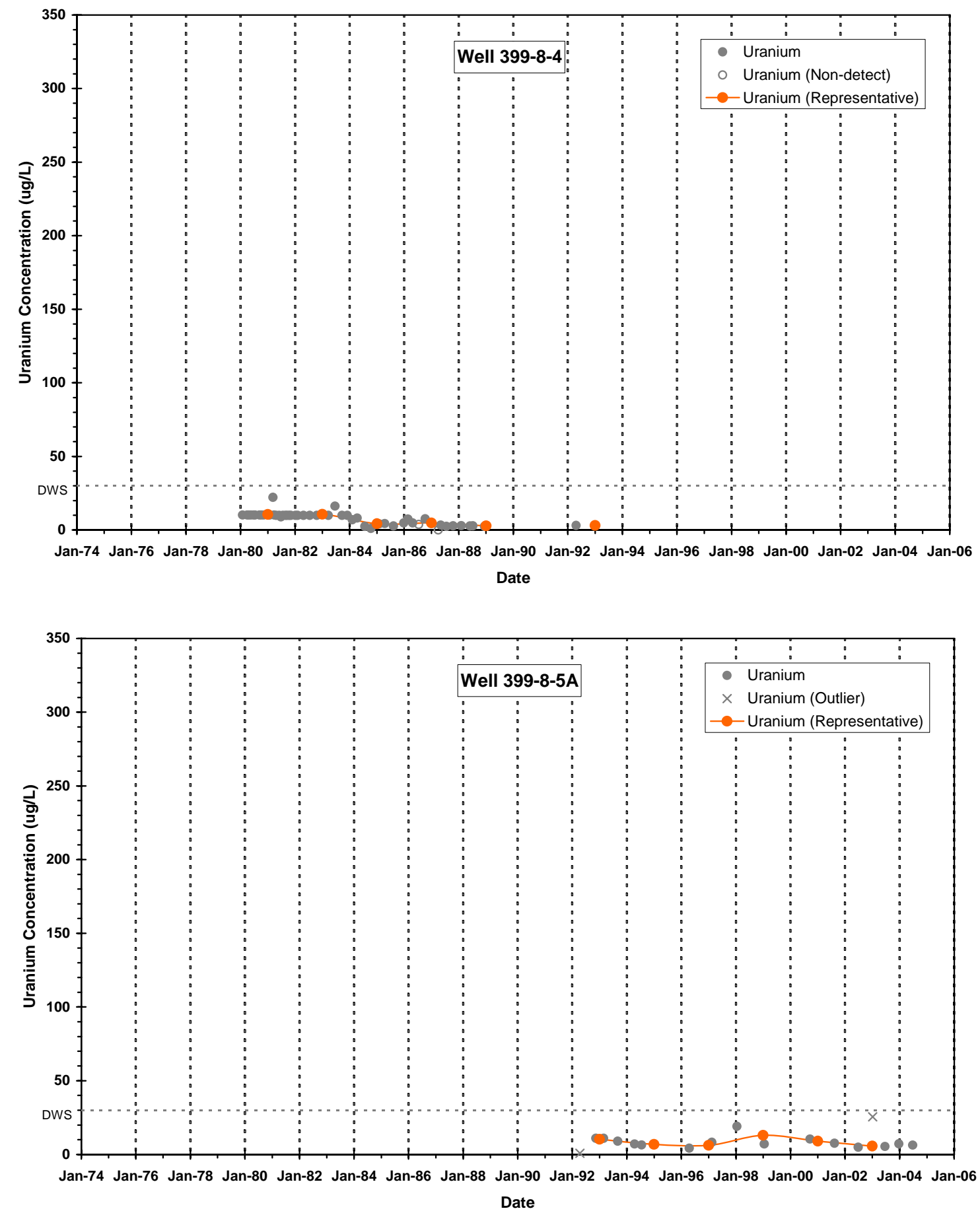

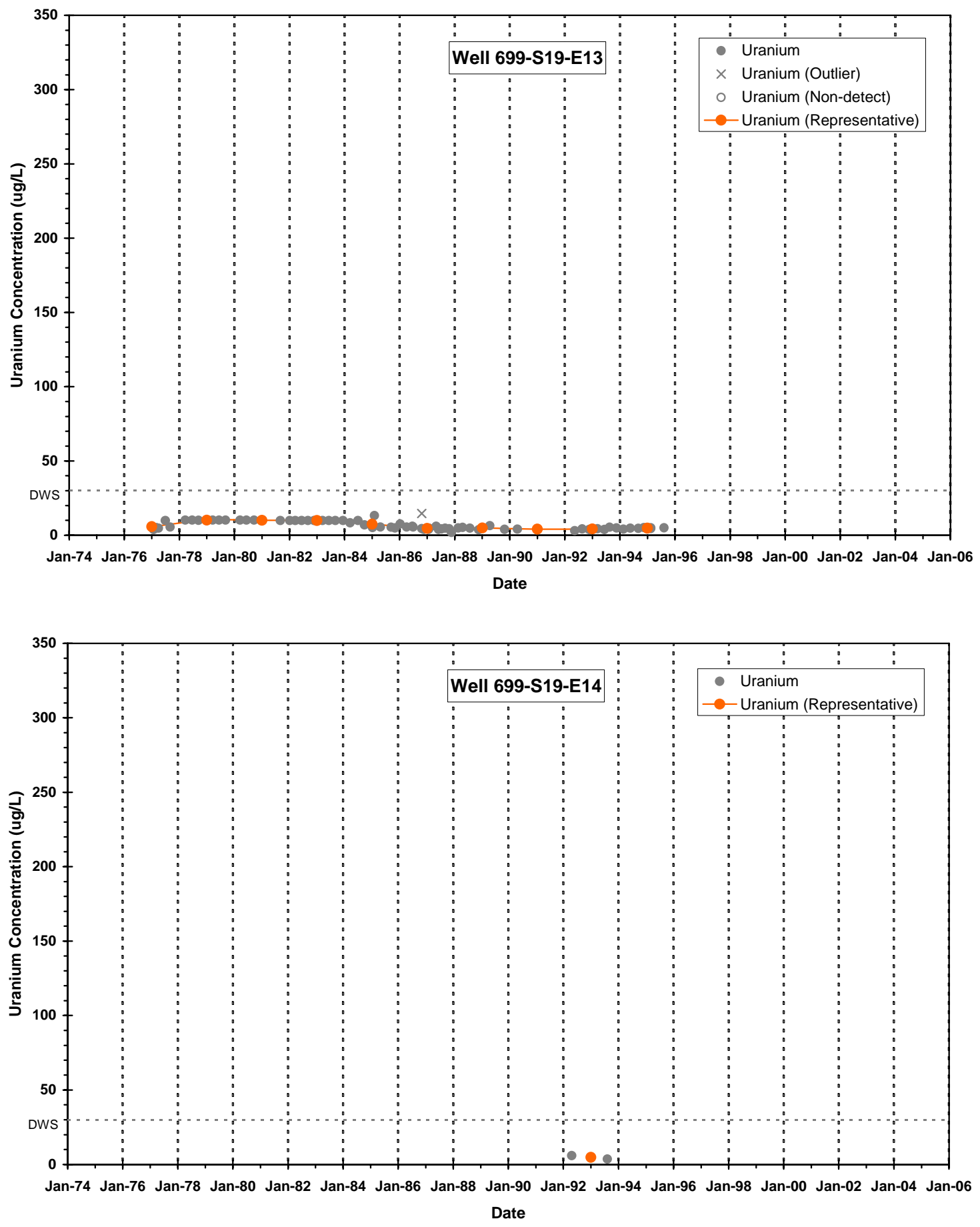


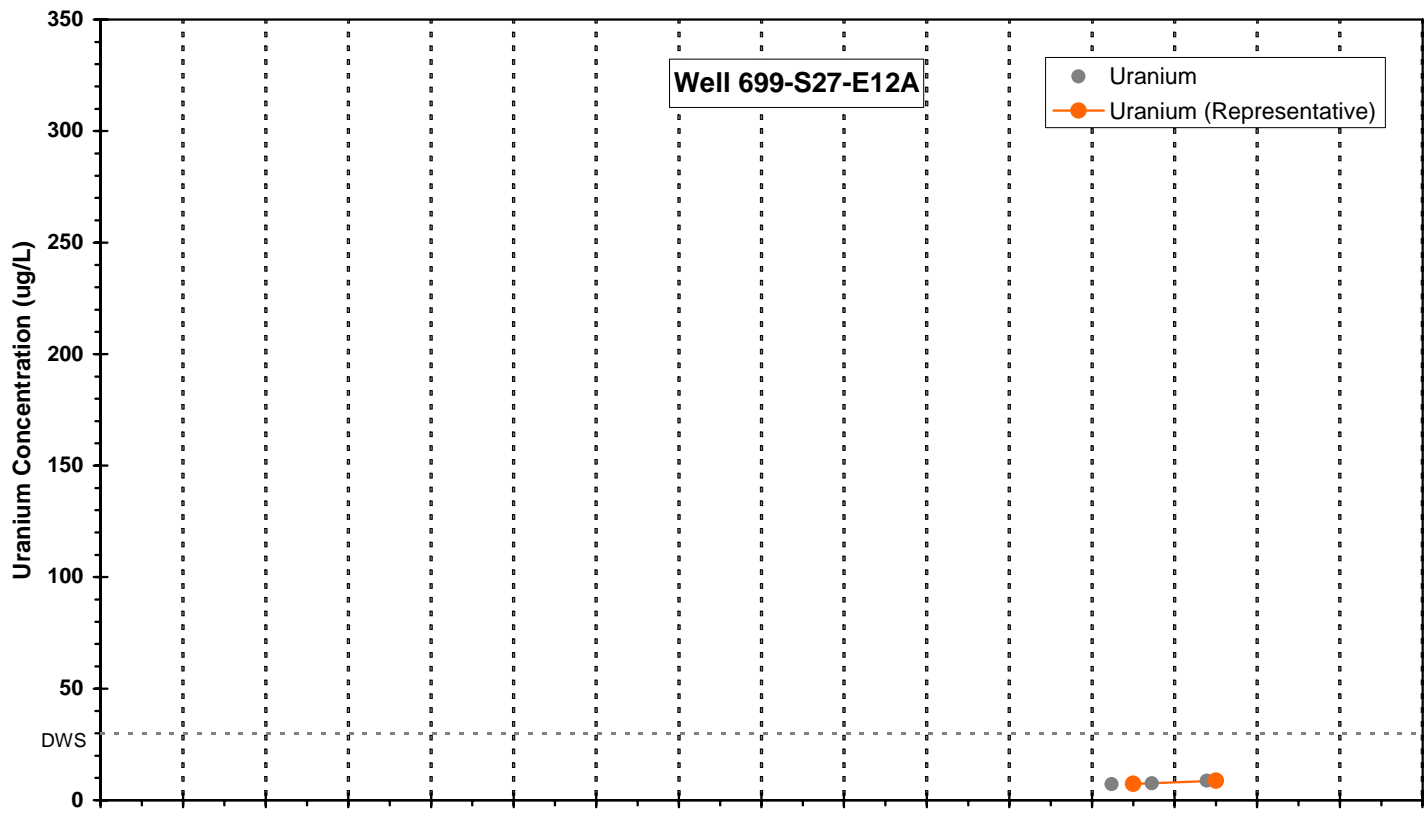

Jan-74 Jan-76 Jan-78 Jan-80 Jan-82 Jan-84 Jan-86 Jan-88 Jan-90 Jan-92 Jan-94 Jan-96 Jan-98 Jan-00 Jan-02 Jan-04 Jan-06

Date

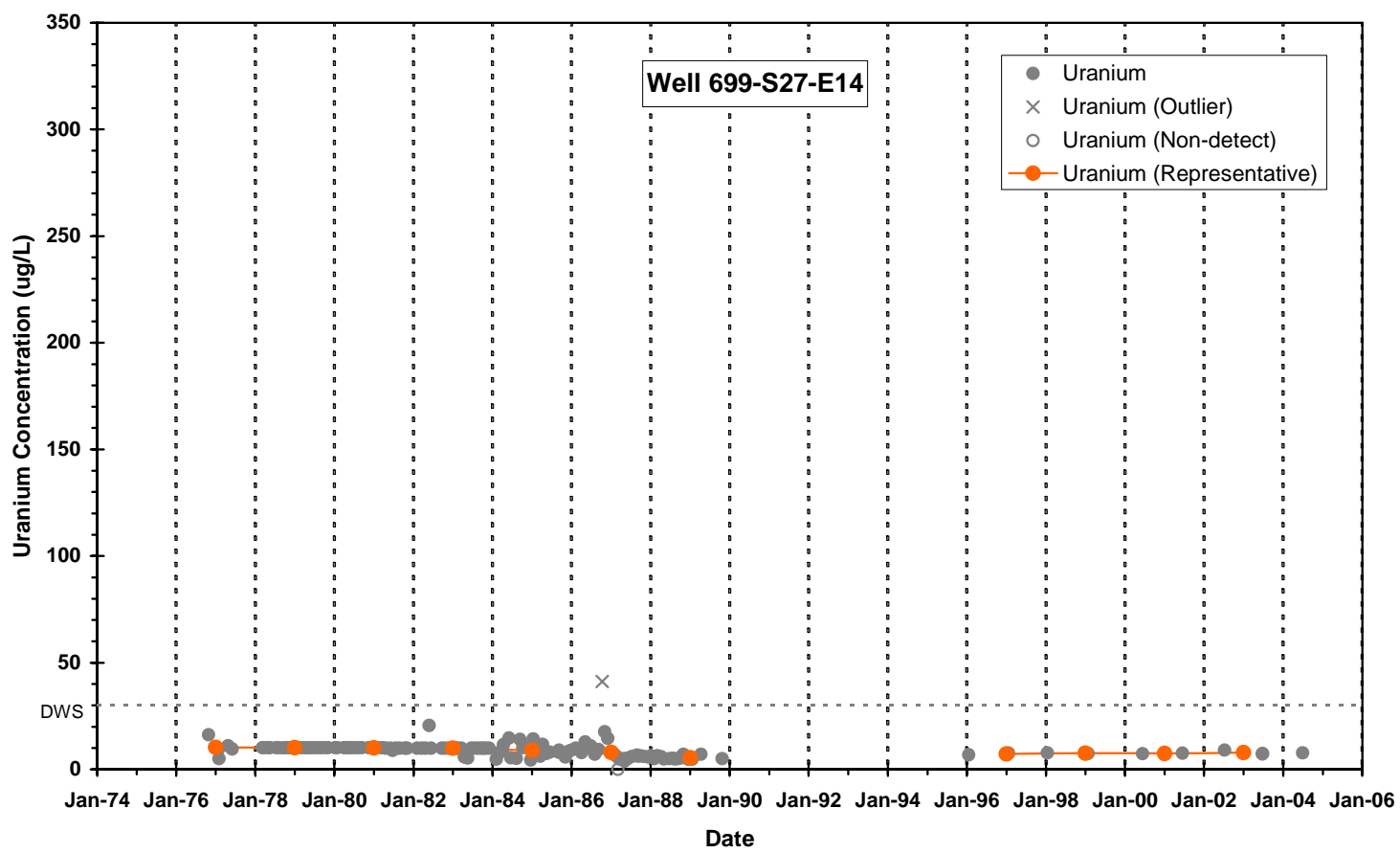



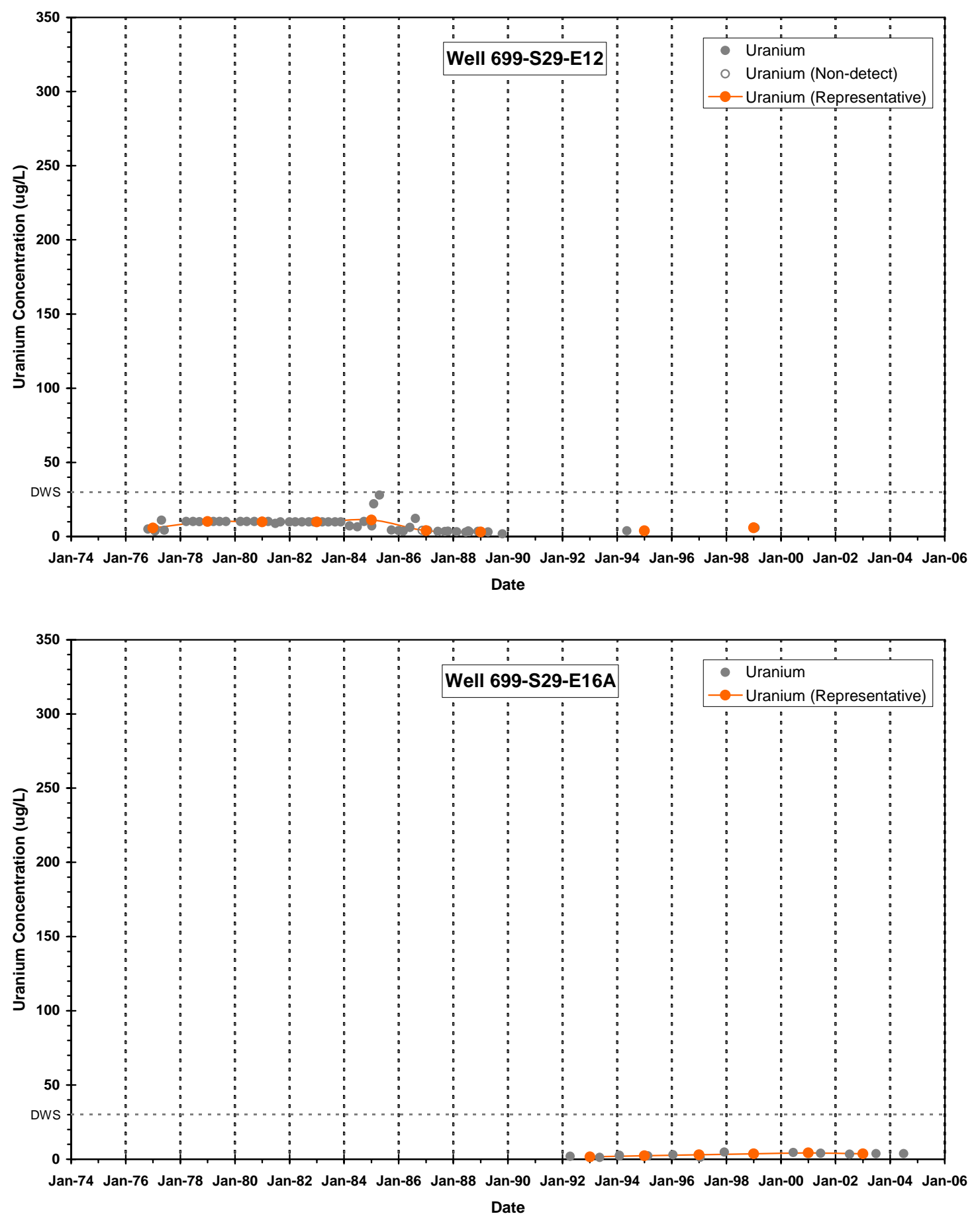


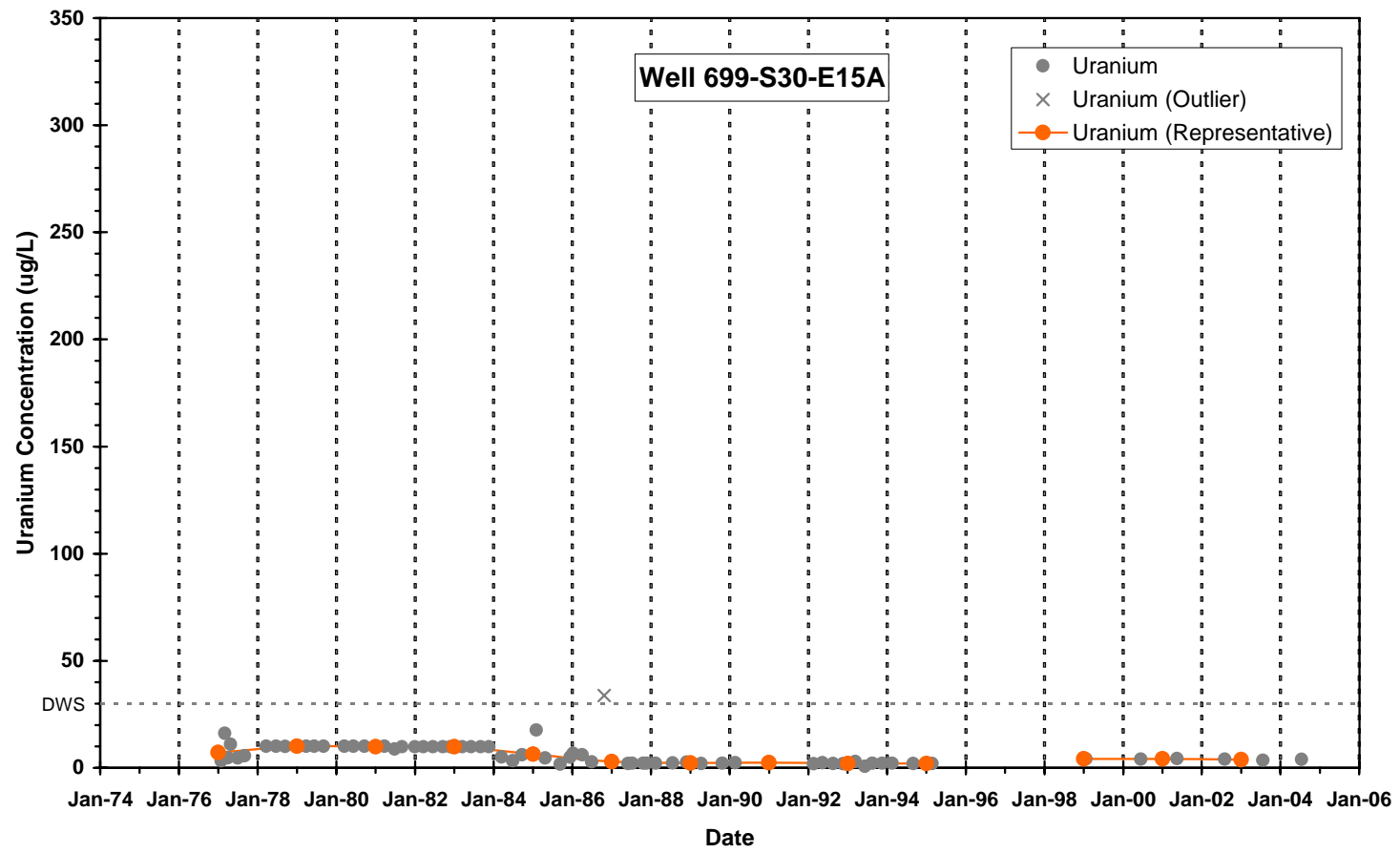




\section{Appendix B}

Tri-Party Agreement Milestone M-016-83, March 11, 2005 


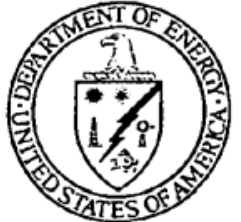

05-AMCP-0188

\section{Department of Energy \\ Richland Operations Office \\ P.O. Box 550 \\ Richland, Washington 99352}

MAR 112005

Mr. Nicholas Ceto, Program Manager

Office of Environmental Cleanup

Hanford Project Office

U.S. Environmental Protection Agency

712 Swift Boulevard, Suite 5

Richland, Washington 99352

Dear Mr. Ceto:

\section{MODIFY THE DESCRIPTION AND COMPLETION DATE FOR HANFORD FEDERAL FACILITY AGREEMENT AND CONSENT ORDER (TRI-PARTY AGREEMENT INTERIM MILESTONE M-016-68 [300-FF-5 OPERABLE UNIT])}

The U.S. Department of Energy, Richland Operations Office (RL) and the U.S. Environmental Protection Agency (EPA) have agreed to re-evaluate remedial action alternatives to meet the remediation goals identified in the July 1996 Record of Decision (ROD) - primarily, the restoration of the aquifer to drinking water standards within a reasonable timeframe.

Tri-Party Agreement Interim Milestone M-016-68 was established for the delivery of a Focused Feasibility Study/Draft Proposed Plan (FFS/PP) in March 2005 to evaluate remediation technology alternatives. The milestone recognizes that treatability tests may be necessary to prepare the FS/PP. A provision was made in the milestone to delay the FFS/PP if such tests were deemed necessary. "If a treatability test is required, a new milestone for delivery of an updated FFS and PP will be negotiated to accommodate the test and assessment of its results." Discussions at the Unit Manager level have resulted in an understanding supporting the delay of the FFS/PP to provide adequate time to perform additional characterization and to perform treatability tests of selected technologies. Therefore, $\mathrm{RL}$ is proposing that Tri-Party Agreement Interim Milestone M-016-68 be modified to reflect the specific deliverables that will be submitted in March 2005, including a work plan for the remaining activities. The proposed remaining work is necessary to address the adequacy of uranium cleanup levels and provide the basis for groundwater remediation decisions. Operable Unit (OU) specific groundwater/contaminant-transport conceptual and numeric models require additional characterization to resolve remaining uncertainties in the conceptual model required to choose and implement a remedial action. Treatability tests will ensure that a technically-sound and defensible PP can be submitted. Significant progress has been made in accomplishing the goal of assessing remedial technologies. RL will submit a document by March 31,2005 , that will include: (1) detailed descriptions of updated conceptual models for the 300 Area uranium plume 
and the 618-11 tritium plume including characteristics and trends for all previously identified contaminants of potential concern (COPC), (2) an evaluation of the COPC and an updated list of COPC with a path forward for further evaluation of the COPC that are retained, and (3) a work plan submitted as a Tri-Party Agreement Primary Document that describes the scope and schedule for activities leading to the FFS/PP, including a recommended Tri-Party Agreement milestone for the FFS/PP. The March 2005 deliverable will provide data necessary to support the upcoming five-year review of 300-FF-5 ROD and the 300 Area End States Workshop.

The enclosed Tri-Party Agreement change package was developed in consultation with U.S. Environmental Protection Agency staff. As described in the Tri-Party Agreement Action Plan, Section 12.0, "Changes to the Agreement." The Tri-Parties have 14 days following receipt of a signed change package to act on the change package.

If you have questions, please contact me, or your staff may contact Matt McCormick, Assistant Manager for the Central Plateau, on (509) 373-9971.

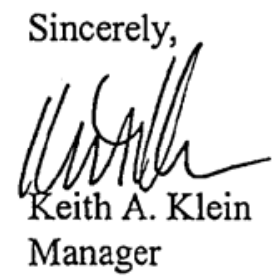
AMCP:KMT
Manager

Enclosure

cc w/encl:

D. Bartus, EPA

G. Bohnee, NPT

L. D. Crass, FHI

L. J. Cusack, Ecology

D. Goswami, Ecology

S. Harris, CTUIR

J. E. Hedges, Ecology

A. Huckaby, Ecology

J. S. Hertzel, FHI

R. Jim, YN

S. Luttrell, PNNL

T. Martin, HAB

E. J. Murphy-Fitch, FHI

K. Niles, ODOE

R. T. Wilde, FHI

B. A. Williams, PNNL

Administrative Record (H6-08) 


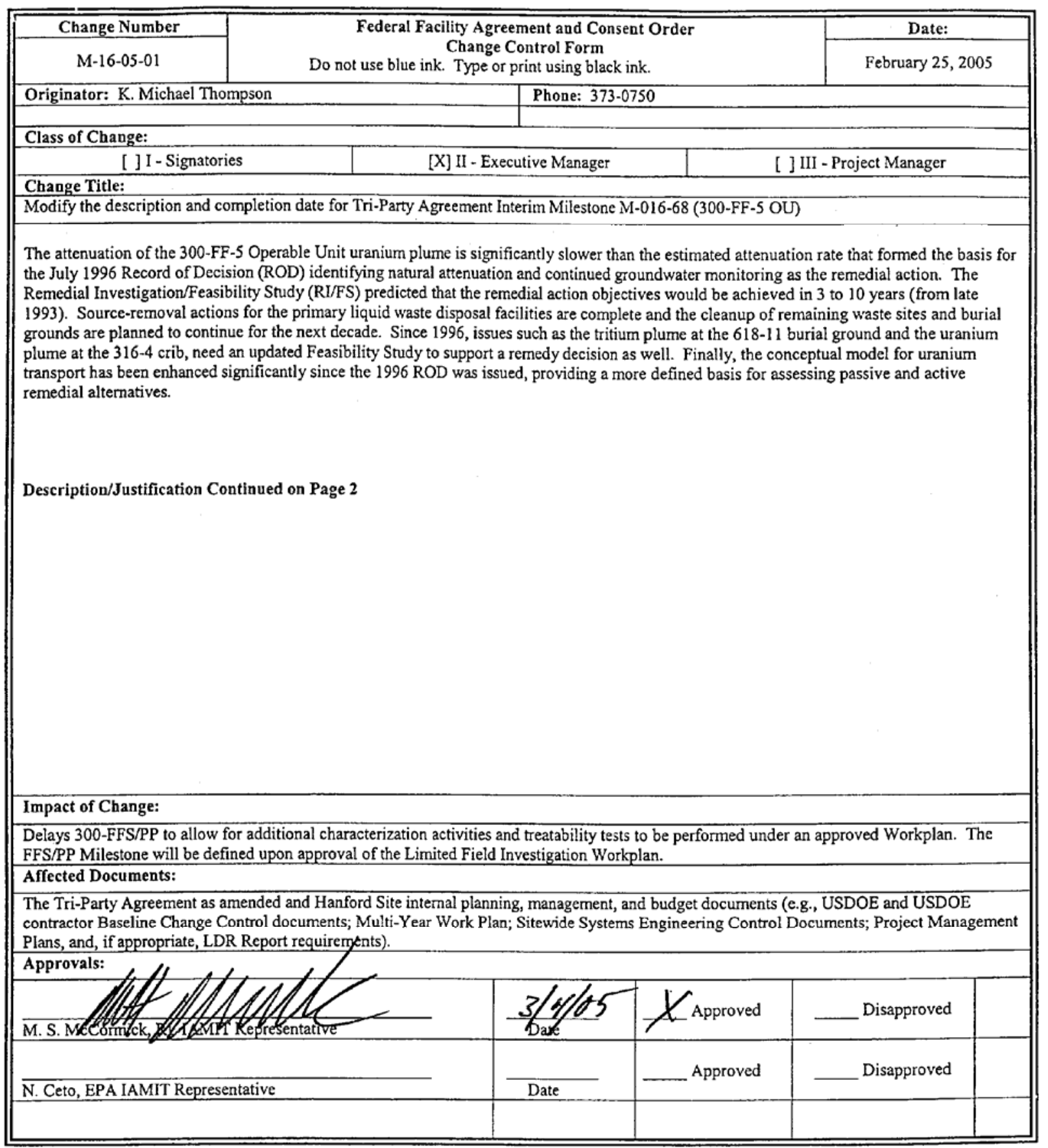


Jiri-Party Agreement Change Request

M-16-05-01

Page 2 of 2

Description/Justification of Change (Continued)

The U.S. Department of Energy, Richland Operations Office (RL) and the U.S. Environmental Protection Agency (EPA) have agreed to re-evaluate remedial action alternatives to meet the remediation goals identified in the July 1996 Record of Decision (ROD) - primarily, the restoration of the aquifer to drinking water standards within a reasonable timeframe.

Tri-Party Agreement Interim Milestone M-016-68 was established for the delivery of a Focused Feasibility Study /Draft Proposed Plan (FFS/PP) in March 2005 to evaluate remediation technology alternatives. The milestone recognizes that treatability tests may be necessary to prepare the FS/PP. A provision was made in the milestone to delay the FFS/PP if such tests were deemed necessary, "If a Treatability test is required, a new milestone for delivery of an updated FFS and PP will be negotiated to accommodate the test and assessment of its results". Discussions at the Unit Manager level have resulted in an understanding supporting the delay of the FFS/PP to provide adequate time to perform additional characterization and to perform treatability tests of selected technologies. Therefore, RL is proposing that Tri-Party Agreement Interim Milestone M-016-68 be modified to reflect the specific deliverables that will be submitted in March 2005, including a limited field investigation work plan for the remaining activities as described below. The proposed remaining work is necessary to address the adequacy of uranium cleanup levels and provide the basis for groundwater remediation decisions. Operable Unit specific groundwater/contaminant-transport conceptual and numeric models require additional characterization to resolve remaining uncertainties in the conceptual model required to choose and implement a remedial action. Treatability tests will ensure that a technically-sound and defensible proposed plan can be submitted.

Significant progress has been made in accomplishing the goal of assessing remedial technologies. RL will submit a document by March 31, 2005 that will include (1) Detailed descriptions of updated conceptual models for the 300 Area uranium plume and the 618-11 tritium plume including characteristics and trends for all previously identified contaminants of potential concern (COPC); (2) An evaluation of the COPC's and an updated list of COPC's with a path forward for further evaluation of the COPC's that are retained; and, (3) A work plan submitted as a Tri-Party Agreement Primary Document that describes the scope and schedule for activities leading to the FFS.PP, including a recommended Tri-Party Agreement milestone for the FFS/PP. The March 2005 deliverable will provide data necessary to support the upcoming five-year review of $300-\mathrm{FF}-5 \mathrm{ROD}$ and the 300 Area End States Workshop.

The FS/PP will be coordinated with ongoing Columbia River risk assessment activities. Additional analysis may be necessary to evaluate technologies for further reducing the flux of risk-driving contaminants to the Columbia River and its riparian habitat.

The proposed change is aligned with M-016-00B major milestone, Complete All Interim Response Actions for the 300 Areas due September 30, 2018.

Modifications established by approval of this Tri-Party Agreement Change Request are denoted as for deletions/modification and șhading for new text.

\begin{tabular}{|c|c|c|}
\hline Milestone & Description & Date \\
\hline M-016-68 & 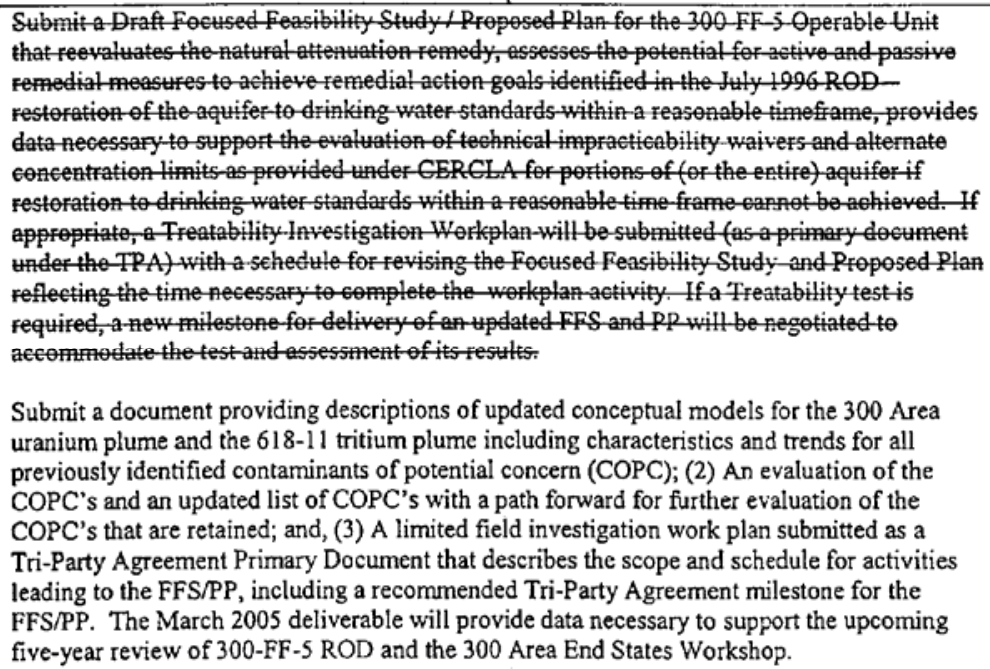 & March 31,2005 \\
\hline
\end{tabular}




\section{Appendix C}

Evolution of Regulatory Framework for 300-FF-5 Operable Unit 


\section{Appendix C}

\section{Evolution of Regulatory Framework for 300-FF-5 Operable Unit}

This section describes the evolution of the regulatory framework for the 300-FF-5 Operable Unit. The current framework results in activities being conducted under a record of decision (ROD) for interim remedial actions, which will continue until cleanup activities associated with the 300-FF-2 Operable Unit are complete, or until other circumstances require an update to the ROD. The current regulatory framework has evolved from an initial ROD for interim remedial action; an explanation of significant difference that adds to the geographical area of the operable unit, the first 5-year review of the ROD, and a revised operations and maintenance plan.

\section{C.1 Record of Decision for 300-FF-1 and 300-FF-5 Operable Units}

The ROD for the 300-FF-1 and 300-FF-5 Operable Units was signed in July 1996 (EPA 1996). At that time, the geographic area covered by the ROD included only the Hanford Site 300 Area. In 2000, groundwater beneath additional waste sites was added to the 300-FF-5 Operable Unit by an Explanation of Significance Differences (EPA 2000). That agreement retained the selected remedies and remedial action objectives (RAO) contained in the original ROD.

In the following paragraphs, text in italics is verbatim from the referenced source. Editorial remarks have been added in brackets for clarity, where necessary.

Declaration of Remedy (EPA 1996, pg. ii)

The selected remedy for 300-FF-5 is an interim remedial action that involves imposing restrictions on the use of groundwater until such time as health-based criteria are met for uranium, trichloroethene, and 1,2-dichloroethene. This is an interim action because there are other constituents (e.g., tritium) which are migrating into 300-FF-5 that have not yet been fully addressed and because a portion of 300-FF-5 is overlaid by uncharacterized waste sites in 300-FF-2. A final remedial action decision for 300-FF-5 will be made after these issues have been addressed. The selected interim remedy includes:

- Continued monitoring of groundwater that is contaminated above health-based levels to ensure that concentrations continue to decrease;

- Institutional controls to ensure that groundwater use is restricted to prevent unacceptable exposures to groundwater contamination.

Implementation of Selected Remedy (GW-2 Institutional Controls with Continued Monitoring): Groundwater Monitoring and Natural Attenuation (EPA 1996, pg. 62)

Continued groundwater monitoring is necessary to verify modeled predictions of contaminant attenuation and to evaluate the need for active remedial measures. 
The monitoring system will be designed and optimized to confirm that attenuation is occurring. The monitoring frequency will be selected to ensure that achievement of the RAO's can be verified. The specific locations and measurements will be documented in an operation and maintenance plan for 300FF-5, which will be approved by EPA. If monitoring does not confirm the predicted decrease of contaminant levels, DOE and EPA will evaluate the need to perform additional response actions. The RI/FS predicted ${ }^{1}$ that the RAOs would be attained in 3 to 10 years. ${ }^{2}$

[1. The prediction only referred to the reduction in uranium concentrations in groundwater, not to all RAO's (ed.).]

[2. The starting date for the predicted interval is 1993 (ed.).]

Remedial Action Objectives for the 300-FF-1 and 300-FF-5 Operable Units (ROD 1996, pg. 51)

1. Protect human and ecological receptors from exposures to contaminants in soils and debris by exposure, inhalation, or ingestion of radionuclides, metals, or organics.

2. Protect human and ecological receptors from exposure to contaminants in the groundwater ${ }^{1}$ and control the sources of groundwater contamination in 300-FF-1 to minimize future impacts to groundwater resources.

[1. ....at levels exceeding drinking water standards... (ed.)]

3. Protect the Columbia River such that contaminants in the groundwater, or remaining in the soil after remediation, do not result in an impact to the Columbia River that could exceed the Washington State Surface Water Quality Standards.

[1. The intent here appears to include preventing further degradation of groundwater such that potential receptors at groundwater discharge locations in the Columbia River are not subject to any additional incremental adverse risks. The latter point suggests that current risks were evaluated during the remedial investigation and found to be acceptable during the period of interim action—uranium was not considered to be an ecological risk driver (ed.).]

\section{C.2 First 5-Year Review of the Record-of-Decision}

The first 5-year review of the 300-FF-1 and 300-FF-5 Operable Units ROD was performed by the EPA Region 10 Hanford Project Office. The triggering date for the review was July 1996, when major excavation activities associated with liquid effluent disposal sites were started. The EPA provided a restatement in the review of the groundwater conclusions upon which the original ROD was based, i.e., the principal assumptions for selecting the interim remedy for groundwater:

Conclusions Upon Which the 1996 ROD Were Based (EPA 2001, pg. 300-9)

- Uranium was the primary contaminant of concern in 300 Area groundwater, although smaller amounts of trichloroethene (TCE) and 1,2-Dichloroethene (DCE) were also detected above action levels.

- 300-FF-1 liquid disposal sites were a primary source of the groundwater contamination. 
- Elevated uranium concentrations in groundwater were estimated to dissipate in 3 to 10 years from late 1993.

- TCE levels were declining below action levels at the time, and DCE was expected to remain in the unconfined aquifer above action levels for "an undetermined period of time." Both compounds were localized.

- Two groundwater plumes are entering the 300 Area from other parts of the Hanford Site. A tritium plume $^{1}$ is entering from the north, and a TCE plume ${ }^{2}$ is entering from the southwest.

[1. The sitewide plume from the 200 East Area contains tritium, nitrate, iodine-129, and technetium-99 (ed.).]

[2. The plume from sources to the southwest contains uranium, trichloroethene, and nitrate (ed.).]

Given this information, the remedy selected [... for groundwater-ed.] was monitored natural attenuation ${ }^{1}$ with institutional controls to prevent human exposure to groundwater. The ROD required continued groundwater monitoring to verify modeled predictions of contamination attenuation and to evaluate the need for active remedial measures. Institutional controls were required to prevent groundwater use while contaminant plumes were still present above drinking water standards.

[1. That is, continued monitoring of conditions and characterization of natural processes that cause attenuation, not a formal monitored natural attenuation remedy (ed.).]

Evaluation of 300-FF-5 Remedy Implementation (EPA 2001, pp. 300-14 and -15)

- It is not clear if the uranium plume in the 300 Area is attenuating, and the O\&M Plan ${ }^{1}$ is not adequate to demonstrate that other plumes in 300-FF-5 are attenuating, either.

[1. Refers to the original operations and maintenance plan, DOE 1996 (ed.).]

- This assessment ${ }^{1}$ will consider the factors described in 'Use of MNA at Superfund, RCRA Corrective Action, and Underground Storage Tank Sites (OSWER 9200.4-17P, April 1999, EPA 1999), and address all groundwater contamination issues identified in the 300-FF-5 Operable Unit, including uranium in the 300 Area and 316-4/618-10 Burial Ground area; TCE, DCE, tetrachloroethene, and strontium-90 in the 300 Area; tributyl phosphate in the 316-4/618-10 Burial Ground area; and tritium at the 618-11 Burial Ground.

[1. The review indicated that not enough information on the attenuation of plumes was being provided to EPA on a regular basis. If evidence exists to show that attenuation is not occurring, then an assessment of active remedial measures must be presented to EPA (ed.).]

- ... groundwater monitoring and soil site investigation/remediation are not being coordinated in the 300 Area.

- ...additional wells or additional samples may be required downgradient of this source [618-4 Burial Ground-ed.] to evaluate groundwater impacts. 
- ...the conceptual site model used to support CVP's [Closure Verification Package-ed.] for soil sites in 300-FF-1 does not conform to the interpretations of groundwater plume fate and transport that have been made by the site-wide monitoring program...

- More extensive river monitoring is needed in this area to evaluate the impact of uranium and other 300 Area contaminants entering the river through groundwater discharge, especially in the vicinity of springs.

Action Item for Groundwater (EPA 2001, pg. 300-15)

DOE shall update and expand the operations and maintenance (O\&M) plan for the 300-FF-5 Operable Unit. The revised O\&M plan shall address:

1. Requirements for monitoring groundwater and river springs in 300-FF-5;

2. Requirements for monitoring any impacts that may be associated with contaminated groundwater and river spring discharges;

3. Requirements for evaluation of groundwater data, including an assessment of the effectiveness of the natural attenuation remedy; and

4. Regulatory reporting requirements.

DOE shall submit a revised O\&M plan by September 2001. DOE shall implement the revised O\&M plan as approved by EPA. [Revised plan (DOE 2002) was approved by EPA and released in May 2002—ed.]

\section{C.3 Revised Operations and Maintenance Plan}

The revised operations and maintenance plan for the 300-FF-5 Operable Unit (DOE 2002) identifies specific topics to be developed during the period of interim action, to provide information for the second 5-year review, and also for evaluating remedial action alternatives. A list of those topics is presented below, along with comments on where related information can be found.

- Evaluate the influence of source removal (i.e., remove-treat-dispose remedy) on groundwater quality

- Closure verification packages for 300 Area source removal sites; RESRAD results.

- Trend charts for contaminant concentrations in groundwater (see Appendix A of this report)

- Evaluate the impact of residual vadose zone contamination on groundwater quality

- Trend charts for contaminant concentrations in groundwater, which show increased concentrations with increased water-table elevations (see Chapter 2 and Appendix A of this report; Hartman et al. 2005, Section 2.12)

- Plume maps that suggest vadose zone hot spots at the 300 Area process trenches and 307 trench (see Chapter 2 of this report)

- Geochemical investigations that indicate long-term, slow release of uranium sorbed onto vadose zone sediment (Zachara et al. 2005) 
- Examine the plume geometry to determine whether high-concentration areas are shrinking, growing, or staying the same over time

- Plume area, volume, and contaminant mass evaluations; geostatistical analysis of plumes (see Chapters 2 and 3 of this report)

- Re-evaluate the contaminants of concern (COC) and well locations to ensure that all significant groundwater contamination sources have been identified and are being monitored

- Summary charts for each contaminant of potential concern (COPC), for each of the 300-FF-5 sub-regions (see Chapter 2 of this report)

- Installation of aquifer sampling tubes at eight sites along the 300 Area shoreline, which provides more comprehensive areal coverage of plume than previous coverage (see Chapter 2 of this report)

- Evaluate $\mathrm{K}_{\mathrm{d}}$ values for all COC, as appropriate

- Geochemical investigations and contaminant transport modeling (Zachara et al. 2005)

- Determine horizontal and vertical extent of COC. If any new wells are drilled, the vadose soils will be sampled as the wells are drilled to determine vertical extent of COCs

- Updated plume maps as new monitoring results are obtained (see Chapter 2 of this report)

- New information on vertical distribution from aquifer sampling tubes (see Figure 2.9 and Chapter 2 of this report)

- Evaluate trend plots of contaminant and predicted degradation product concentrations to determine if the COC are behaving consistently with predicted natural attenuation scenarios

- Summary charts for each COPC, for each of the 300-FF-5 sub-regions (see Chapter 2 of this report)

- New predictions for uranium plume will evolve from groundwater flow and contaminant transport modeling tasks that are underway (projects underway under Remediation Science and Technology Project)

- Update existing site conceptual contaminant fate and transport models, or develop new models to predict attenuation rates, as necessary

- Updated 3-D hydrogeologic model, to be used as the spatial framework in several computer simulation models for groundwater flow and uranium transport (see Chapters 3 and 4 of this report)

- Consider additional remedial actions if MNA is determined to be ineffective

- Revisiting initial feasibility study of remedial action alternatives for uranium (work plan available March 31, 2005, under TPA milestone M-016-83)

- Consider a technical impracticability waiver under CERCLA if none of the remedial alternatives prove practicable

- $\quad$ Proposed plan for future activities at the 300-FF-5 Operable Unit; tbd) 


\section{C.4 References}

DOE. 1996. Operation and Maintenance Plan for the 300-FF-5 Operable Unit. DOE/RL-95-73, Rev. 0. Prepared by CH2M HILL Hanford, Inc. for the U.S. Department of Energy, Richland, Washington.

DOE. 2002. Operation and Maintenance Plan for the 300-FF-5 Operable Unit. DOE/RL-95-73, Rev. 1. Prepared by CH2M HILL Hanford, Inc. for the U.S. Department of Energy, Richland, Washington.

EPA. 1996. Record of Decision for USDOE Hanford 300-FF-1 and 300-FF-5 Operable Units Remedial Actions. Agreement Between U.S. Department of Energy and U.S. Environmental Protection Agency, with Concurrence by the Washington State Department of Ecology, July 17, 1996.

EPA. 1999. Use of Monitored Natural Attenuation at Superfund, RCRA Corrective Action, and Underground Storage Tank Sites. OSWER Directive 9200.4-17, U.S. Environmental Protection Agency, Washington, D.C.

EPA. 2000. Explanation of Significant Difference for the 300-FF-5 Record of Decision (see EPA 1996 for original ROD). Agreement between U.S. Department of Energy and U.S. Environmental Protection Agency, with concurrence by the Washington State Department of Ecology, June 2000. (adds groundwater beneath the 618-10 and 618-11 Burial Grounds to the 300-FF-5 Operable Unit)

EPA. 2001. USDOE Hanford Site: First Five Year Review Report. April 2001. Prepared by U.S. Environmental Protection Agency, Region 10, Hanford Project Office, Richland, Washington.

Hartman MJ, LF Morasch, and WD Webber (eds.). 2005. Hanford Site Groundwater Monitoring for Fiscal Year 2004. PNNL-15070, Pacific Northwest National Laboratory, Richland, Washington.

Zachara, JM, JA Davis, C Liu, JP McKinley, N Qafoku, DM Wellman, and SB Yabusaki. 2005. Uranium Geochemistry in Vadose Zone and Aquifer Sediments from the 300 Area Uranium Plume. PNNL-15121, Pacific Northwest National Laboratory, Richland, Washington. 


\section{Distribution}

No. of

Copies

ONSITE

13 DOE Richland Operations Office

BL Charboneau

RD Hildebrand (3)

JG Morse

KM Thompson (3)

AC Tortoso

Public Reading Room (2)

Administrative Record (2)

2 Bechtel Hanford, Inc.

JA Lerch

SG Weiss

3 Fluor Hanford, Inc.

JV Borghese

BH Ford

VG Johnson

2 Washington State Department of Ecology

FW Bond

J Price
L6-06

E6-35

E6-35

H0-57

A6-33

A6-38

A6-38

A6-38

A6-38

H2-53

H6-08

H0-23

H0-57
No. of

Copies

2 U.S. Environmental Protection Agency

DA Faulk

B5-01

LE Gadbois

B5-01

25 Pacific Northwest National Laboratory

PE Dresel

K6-96

EJ Freeman

K9-36

JS Fruchter

K6-96

TJ Gilmore

K6-81

MJ Hartman

K6-96

NP Kohn

JW Lindberg

SEQUIM

K6-81

K6-96

K6-96

JP McDonald

K6-81

CJ Murray

TG Naymik

K6-96

GW Patton

K6-75

RE Peterson (6)

K6-96

JR Serne

P7-22

K9-33

K6-96

MJ Truex

K6-96

VP Vermeul

K9-36

MD Williams

K9-36

JM Zachara

K8-96 